\title{
AN INVESTIGATION INTO THE APPLICATIONS \\ OF POLYMER GEL DOSIMETRY IN \\ RADIOTHERAPY
}

Ali Reza Farajollahi

A thesis submitted for the degree of Doctor of Philosophy

Department of Medical Physics,

Faculty of Medicine,

University of Leicester

1998 
To Mehri and Mehran 


\section{TABLE OF CONTENTS}

Abstract. iv

Acknowledgement. ..v

Statement of Originality................................................................................................

List of figures. ..vii

List of tables xii

Chapter 1. Modern developments in radiotherapy $.1-1$

1.1 Introduction $.1-1$

$1.2 \mathrm{CT}$ in radiotherapy planning .......................................................................1-2

$1.3 \mathrm{MRI}$ in radiotherapy planning......................................................................1-2

1.4 Dosimetry for modern radiotherapy.............................................................

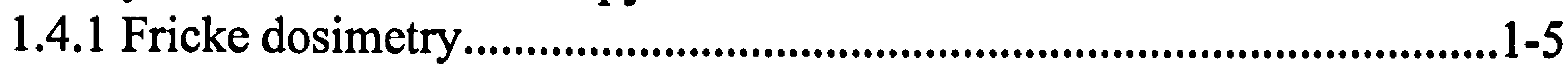

1.5 MRI In Radiotherapy Dosimetry ................................................................1-6

1.5.1 Fricke gel dosimetry....................................................................1-6

1.5.1.1 Choice of dosimeter.....................................................................1-11

1.5.2 Polymer gel Dosimetry.........................................................................1-13

1.6 Aims of the investigation ............................................................................1-16

Chapter 2. Basic theory of MRI.........................................................................................2-1

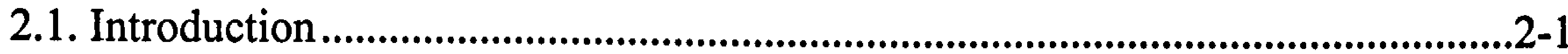

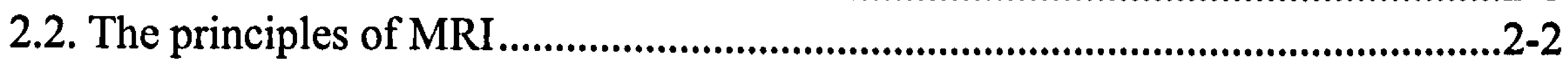

2.3. Physical basis ...........................................................................................2-3

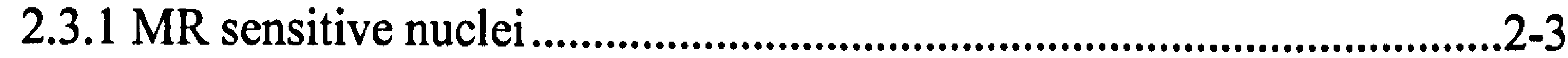

2.3.2 Precession.........................................................................................2-4

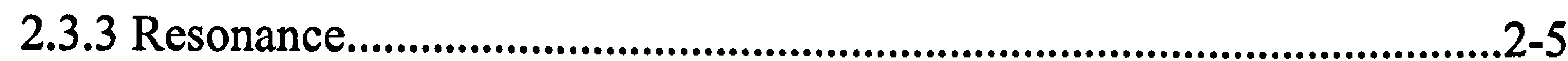

2.3.4 Relaxation....................................................................................2-6

2.3.4.1 Longitudinal relaxation time ................................................2-8

2.3.4.2. Transverse relaxation time ...................................................2-9

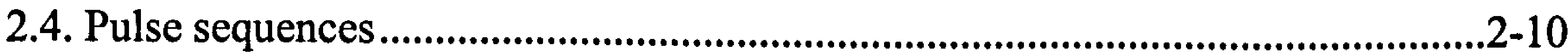

2.4.1 Saturation recovery ..........................................................................2-12

2.4.2 Spin-Echo sequence ..........................................................................2-13

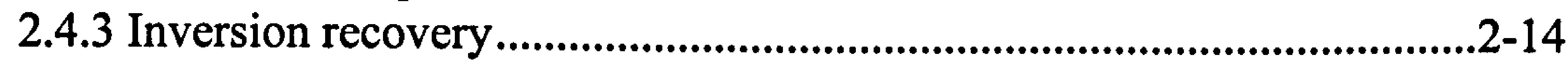

2.5 Relaxation measurements ...............................................................................2-15

2.5.1 Measurement of $\mathrm{T}_{1}$ relaxation time...............................................2-15

2.5.2 Measurement of $\mathrm{T}_{2}$ relaxation time.................................................2-16

2.6. NMR Imaging .................................................................................................2-18

2.6.1 Gradient fields ........................................................................................2-19

2.6.2 Image Acquisition and reconstruction................................................2-21

2.6.2.1 Projection reconstruction........................................................2-21

2.6.2.2 Two-dimensional Fourier Transformation ...............................2-23 


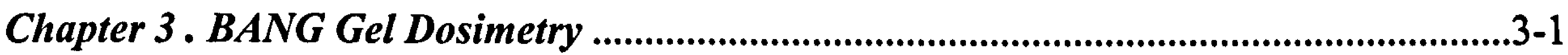

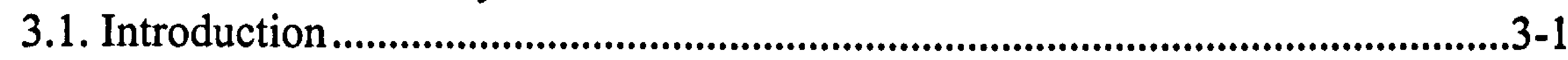

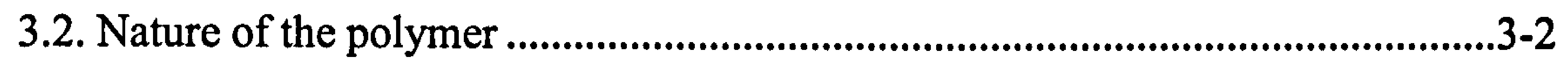

3.3. Properties of the polymer gel ........................................................................3-3

3.4. Radiation-induced polymerization.......................................................................3-4

3.4.1. Kinetics of radiation polymerization..................................................3-5

3.4.2. Cross-linking .........................................................................................

3.5. Considerations in the choice of phantom............................................................3-7

3.6. Methods and Materials...................................................................................

3.6.1. Preparation of BANG gel.................................................................3-9

3.6.2. Effect of oxygen ...................................................................................

3.6.3 Measurement of the samples.........................................................3-12

3.7. Basic properties of the BANG gel ..............................................................3-14

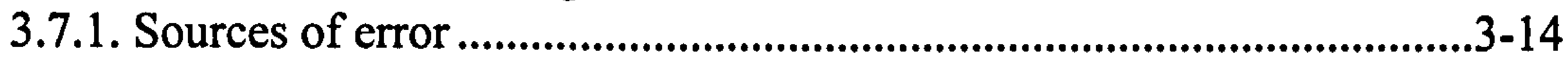

3.7.1.1. Reproducibility of $\mathrm{R}_{2}$ measurements .................................3-14

3.7.1.2. Positional and volume related errors ..................................3-16

3.7.2. Reproducibility of the dose response .................................................3-20

3.7.3. Tissue equivalence .............................................................................3-25

3.7.4 Effect of monomers and gelatin concentration ........................................3-28

3.7.5. Effect of beam energy .............................................................................3-33

3.7.6. Dose rate dependence......................................................................3-35

3.7.7. Diffusion...............................................................................................3-36

3.7.8. Temperature effect ........................................................................3-37

3.7.9. Effect of the time of imaging after irradiation ......................................3-38

3.7.10. Effect of imaging field strength on the measurement $R_{2} \ldots \ldots \ldots \ldots . . . . . .3-40$

3.7.11. Measurement of absolute dose .........................................................3-41

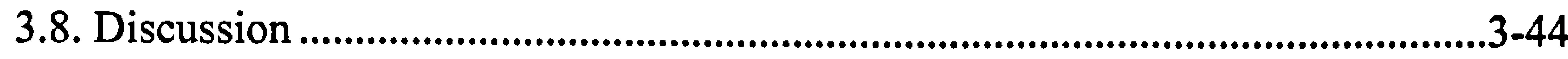

Chapter 4. Application of BANG gel in brachytherapy.....................................................4-1

4.1. Introduction ......................................................................................................4-1

4.2. Methods and Materials..........................................................................................4-2

4.2.1. MRI Imaging and data analyss.......................................................4-2

4.2.2. Overall uncertainty in the dose calculation............................................4-3

4.2.3. Simple LDR brachytherapy..................................................................4-4

4.2.4. Complex LDR brachytherapy .......................................................4-5

4.3. Results and discussion ......................................................................................

4.3.1. Simple LDR brachytherapy.............................................................4-7

4.3.2. Complex brachytherapy ........................................................................4-11

Chapter 5. The application of gel dosimetry in external beam therapy ................................5-1

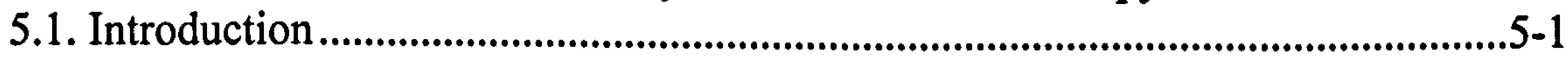

5.2. Single field irradiation (Depth dose determination) ...........................................5-3

5.2.1 Results ......................................................................................................5-4

5.3. Three field irradiation .........................................................................................5-7

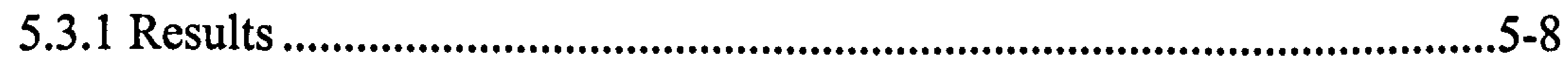

5.4. Four field irradiation ..................................................................................11 


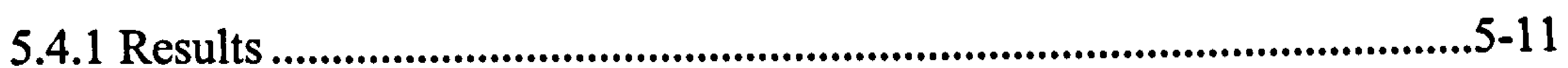

5.5. The dose distribution in an inhomogeneous gel..................................................5-12

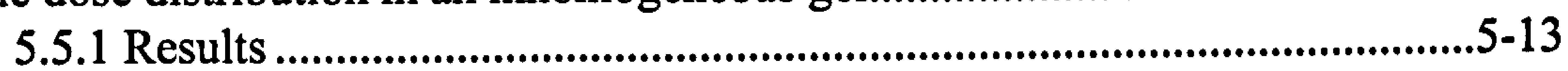

5.6. Oblique beam ...............................................................................................5-15

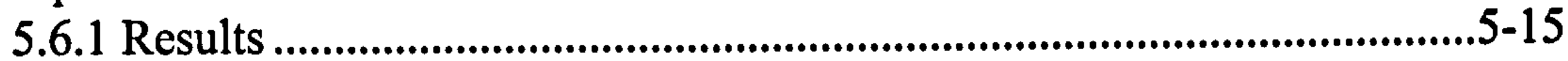

5.7. Irradiation with intensity modulated beams..........................................................5-17

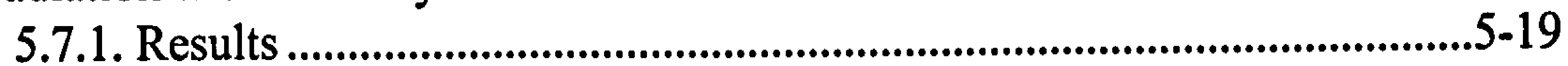

5.8. Dynamic wedge therapy..................................................................................5-21

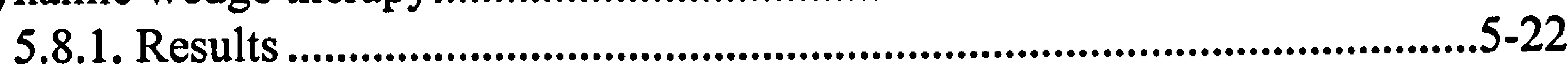

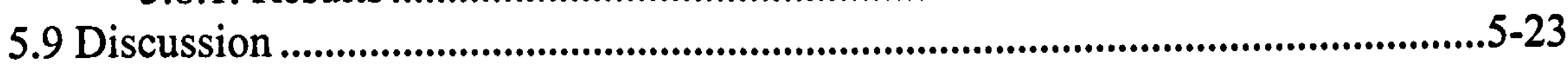

Chapter 6. Other applications of the BANG polymer gel .......................................................6-1

6.1. Boron neutron capture therapy (BNCT) ....................................................................6-1

6.1.1. General background .................................................................................6-1

6.1.2. An accelerator based epithermal neutron beam ........................................6-2

6.1.3. Dosimeter considerations .......................................................................6-3

6.1.4. Methods and materials .......................................................................6-3

6.1.5. Results and discussion........................................................................6-4

6.2. Dose distribution from a blood irradiator ................................................................6-9

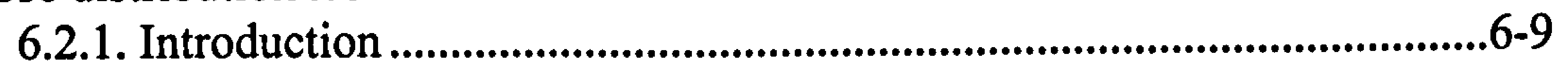

6.2.2. Blood irradiators.................................................................................6-10

6.2.3. Measurement process .............................................................................6-12

6.2.4. Results and discussion..........................................................................6-13

Chapter 7. Conclusions and future work ..........................................................................

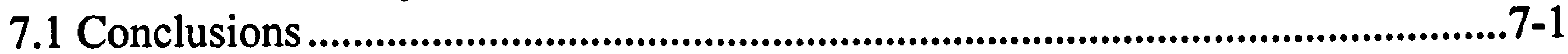

7.2 Future work ................................................................................................7-4

7.3 Practical considerations.......................................................................................

Chapter 8. References ............................................................................................... 


\section{Abstract}

The verification of complex dose distributions produced using novel techniques in radiotherapy requires measurements in three dimensions with high spatial resolution. Recent developments in polymer gel dosimetry employing MRI suggest this may be the best available method.

In this work the properties of a polymer gel (BANG) based on acrylamide and bis-acrylamide were investigated. The gel was found to be tissue equivalent and its response to absorbed dose reproducible to within $\pm 4 \%$ and linear up to $10-12 \mathrm{~Gy}$. The linearity can be increased by using a higher weight fraction of bis-acrylamide. The response of the gel was also found to be independent of energy and doserate, but dependent on oxygen contamination and gel temperature during MR imaging.

The potential usefulness of BANG gel in different areas of radiotherapy was determined. In brachytherapy a comparison of the relative doserate distributions between gel, calculation and TLDs agreed to within $\pm 5 \%$. The overall precision in measurements of absorbed dose was estimated to be $\pm 5 \%$. The measured dose distribution from complex brachytherapy agreed well with the distribution produced by the Helax-TMS planning system. For external beam irradiation the depth doses measured in the gel showed good agreement with ion chamber measurements to within $\pm 3 \%$. The dose distributions produced in the gel from multi-field irradiations were in agreement to within $\pm 4 \%$ at the $50 \%$ isodose level. The gel was also used to verify the absorbed dose distribution produced by intensity modulated beam techniques using compensators. The results of the gel measurements agreed well with film dosimetry. BANG gel was also found to be an excellent candidate for dynamic measurements and measurements in areas with restricted access. A novel technique for measurements in boron neutron capture therapy using the gel was also investigated. It was concluded that gel can be used to verify complex dose distributions. 


\section{Acknowledgement}

I am in debt to those who have contributed in many ways to this work. Firstly I wish to express my gratitude to my supervisor Dr David Bonnett for his enthusiastic support and advice throughout course of this work and also the Iranian Ministry of Health and Medical Education, Tabriz University of Medical Science for financial support, without which it was not possible to carry out this study. I also wish to express my appreciation to Professor Evans for his support. Thanks are also due to Roger Aukett for his interest and valuable discussions, and Dr Ronny Panerai for his concern and encouragement.

During the years of study I have enjoyed invaluable technical support from all the staff of the Departments of Medical Physics, Radiotherapy and MRI of Leicester Royal Infirmary. I would like to thanks all of them in particular Steve Bolton, John Brooks, Glen Bush, Sunil Champaneri, Liz Ching, Andrew Glendinning, David Heaton, Peter Harding, Jilly Hepplewhite, Helen Hill, Derek Jones, Troy Johnson and Mike Squires.

In addition I would like to acknowledge the staff at several departments in the Midlands who have made their irradiation facilities available to me. These include $\mathrm{Dr}$ Stewart Green and Dr David Tattam at Birmingham University, Dr John Mills and Alisa Ratcliffe at the Walsgrave Hospital Coventry and Mrs Sally Powley at Lincoln.

To all my friends I give my thanks for their contributions especially Marco Von Kruger for his contribution to my Brazilian accent!, Dr Lingki Fan for his concern, Mohammad Reza Atemadi for his kindness and all invaluable support, Stephanie Deverson, Vicky Hudson and Marie Sendal for their interest.

Last but not least I wish to express my sincere thanks to my family in particular my mother who has been great support for me, my wife for her love and patience, my son for his forbearance while the work was completed. 


\section{STATEMENT OF ORIGINALITY}

I certify that the following thesis is based on the results of investigations performed by me, that this is my own composition, and that it has not previously been presented for a higher degree.

A.R. Farajollahi 


\section{LIST OF FIGURES}

Chapter 2

Figure 2.1. The net magnetization in the direction of the external field

Figure 2.2. Precession

Figure 2.3. Magnetization (a) at equilibrium, (b) after a $90^{\circ}$ pulse

Figure 2.4. Variation in the net components of the magnetic moment with time

Figure 2.5. Transverse $\left(\mathrm{T}_{2}\right)$ relaxation. (a) After $90^{\circ}$ pulse, $t=0$; (b) at time $\cong \mathrm{T}_{2} ;(\mathrm{c}) \mathrm{t}$ $\rightarrow \infty$

Figure 2.6. The short $T_{1}$ sample produces a larger $N M R$ signal than the long $T_{1}$ sample for the same TR

Figure 2.7. Spin echo-pulse sequence

Figure 2.8. Inversion recovery pulse sequence

Figure 2.9. The CP pulse sequence

Figure 2.10. The CPMG pulse sequence

Figure 2.11. Magnetic field gradient

Figure 2.12. The $\mathrm{X}, \mathrm{Y}$ and $\mathrm{Z}$ gradient axes

Figure 2.13. Selection of the slice in the presence of a field gradient in the $\mathrm{Z}$ direction

Figure 2.14. A spin-echo sequence in the projection reconstruction technique

Figure 2.15. A spin echo sequence in the 2DFT imaging technique

Chapter 3

Figure 3.1. Inhibitive effect of the oxygen on the gel response to radiation

Figure 3.2. Effect of slice thickness on SNR

Figure 3.3. Position of the vials inside the head coil where the signals are collected

Figure 3.4. $\quad T_{2}$ image of the phantom showing the position of the gel vials inside the phantom

Figure 3.5. Set up for radiation beam calibration

Figure 3.6. Photograph of the irradiated gel 
Figure 3.7. The dependence of the transverse relaxation rate, $R_{2}$ on dose in three separately prepared gels over the range from 0 to $15 \mathrm{~Gy}$

Figure 3.8. The linearity of the dose response of the BANG gels over the dose range from 0 to $10 \mathrm{~Gy}$

Figure 3.9. Linearity of the $\mathrm{R}_{2}$ measurements to dose up to $12 \mathrm{~Gy}$

Figure 3.10. Effect of the different chemical batch on the gel response

Figure 3.11. Diagram showing set up for beam calibration

Figure 3.12. Dose response curves obtained with different total monomer concentration

Figure 3.13. Dose response curve obtained from $R_{2}$ measurement in the gel with $4 \%$ of bis

Figure 3.14. The effect of the continuous and pulsed radiation on the gel response

Figure 3.15. Variation of the gel response with doserate

Figure 3.16. Diffusion of the polymer with time

Figure 3.17. Temperature effect on $\mathrm{R}_{2}$ measurement

Figure 3.18. Variation of the gel response with time

Figure 3.19. Stability of the gel response with time

Figure 3.20. Comparison of the different method of calibration

\section{Chapter 4}

Figure 4.1. The phantom with applicator in central Perspex tube

Figure 4.2. The jig used for irradiation of the TLDs. The jig consists of two stacks supported by the Perspex tubes

Figure 4.3. The experimental set up for complex brachytherapy

Figure 4.4. A typical $\mathrm{T}_{2}$ weighted image $(\mathrm{TR}=6000 \mathrm{~ms}$ and $\mathrm{TE}=400 \mathrm{~ms})$ of the calibration vials demonstrating the different signal intensity due to different radiation dose

Figure 4.5. A typical calibration curve for the gel. The error of $3.5 \%$ is indicated for each data points. The linear data fit is $\mathrm{R}_{2}=1.54+0.23 \mathrm{D}, \mathrm{r}=0.996$ 
Figure 4.6. Typical normalized dose distribution of the irradiated gel. The scale shows the percentage of maximum dose. The image shows the sagittal plane. The channel in the middle of the image represents the position of the applicator

Figure 4.7. Isodose levels produced in the irradiated gel in the coronal plane

Figure 4.8. Absolute doserate measured at different distances from the centre of the applicator

Figure 4.9. Relative dose normalized to $3.5 \mathrm{~cm}$ from the centre of the source train

Figure 4.10. An image of the dose distribution produced using the Gynaecological applicator

Figure 4.11. Comparison of isodose between gel and Helax-TMS

\section{Chapter 5}

Figure 5.1. Experimental set up for ion chamber measurement

Figure 5.2. Dose distribution of single $8 \times 8$ radiation in the gel

Figure 5.3. Horizontal profile taken at approximately $\mathrm{d}_{\max }$ in the gel irradiated by $8 \times 8$ $\mathrm{cm}^{2}$ radiation field

Figure 5.4. The comparison of a central axis percentage depth dose curve for $5 \times 5 \mathrm{~cm}^{2}$ field size using ion chamber and gel dosimeter. The data are normlized to a depth of $5 \mathrm{~cm}$

Figure 5.5. The central axis percentage depth dose curve derived from figure 5.2 and its comparison using an ion chamber for $8 \times 8 \mathrm{~cm}^{2}$ field size. The data are normalized to a depth of $5 \mathrm{~cm}$

Figure 5.6. A typical image of regret produced using Pips. The scale represents the percentage of the dose at isocentre.

Figure 5.7. A typical dose distribution resulted from three field treatment using BANG gel in a glass walled phantom. The scale represents the percentage of the normalized value

Figure 5.8. Comparison of a three field treatment plan between Helax-TMS and the polymer gel 
Figure 5.9. Comparison of absorbed dose distributions measured in glass and Perspex walled phantom

Figure 5.10. Comparison of profiles in glass and Perspex phantoms. The profiles were measured along the central axis of one beam of the three field treatment

Figure 5.11. The MRI produced dose map in the gel irradiated to four field treatment

Figure 5.12. Comparison of a four field treatment plan using Helax-TMS with measurement with a polymer gel

Figure 5.13. A typical dose distribution resulted in the irradiated gel with an air inhomogeneity inside for $6 \times 6 \mathrm{~cm}^{2}$ radiation field. Note that there is a slight offset between the beam and the air-filled central cylinder.

Figure 5.14. Comparison of dose distribution produced in the gel with an air gap included and Helax-TMS for $4 \times 4 \mathrm{~cm}^{2}$ field, irradiated using a $6 \mathrm{MV}$ beam

Figure 5.15. Isodose level comparison using gel having an air gap inside and HelaxTMS for $6 \times 6 \mathrm{~cm}^{2}$ radiation field using a $8 \mathrm{MV}$ beam

Figure 5.16. Isodose comparison between the gel in Perspex phantom and the plan produced by Helax-TMS

Figure 5.17. Comparison of dose distribution produced in the gel using glass phantom and the plan produced by Helax-TMS

Figure 5.18. Diagram shows the test case with prescribed treatment area (PTA) which is supposed to represent the prostate and two OAR

Figure 5.19. Schematic diagram shows the gel inside the anthropomorphic phantom with the 5 field treatment using customised compensators

Figure 5.20. predicted dose distribution in aim of sparing organs at risk

Figure 5.21. Dose distribution obtained from irradiation of the film using conformal treatment scheme

Figure 5.22. Dose distribution produced in the gel by conformal treatment and comparison of the $90 \%$ isodose level in the gel and film with calculation

Figure 5.23. Sagittal slice of the gel irradiated using conformal treatment scheme 
Figure 5.24. A topographic plot of dose distribution derived from the polymer gel irradiated by a dynamic wedge with a nominal angle of $45^{\circ}$

Figure 5.25. Dose distribution derived from MRI of the gel phantom taken along the beam axis

\section{Chapter 6}

Figure 6.1. Experimental set-up

Figure 6.2. The dose response of pure and boronated gel to $6 \mathrm{MV}$ x-ray beam

Figure 6.3. MCNP calculation of variation of neutron flux in the water phantom

Figure 6.4. The dose profiles of the first and second vials for both boron mixed and pure gel

Figure 6.5. Relative doses obtained at different depths from the vials filled with pure and boron loaded gel

Figure 6.6. Comparison of the absorbed dose resulting from MCNP calculation with dose measured in pure and boron loaded gel

Figure 6.7. A typical isodose curve supplied by a manufacturer (Atomic Energy of Canada Ltd)

Figure 6.8. Absorbed dose distributions measured in the gel phantom 


\section{LIST OF TABLES}

1.1 Typical gel concentration of the NMR Fricke dosimetry

3.1 Elemental composition of muscle and polyacrylamide gel (\% by weight)

3.2 Reproducibility of $\mathrm{R}_{2}$ measurements at different doses (quoted uncertainties are \pm 1

SD)

3.3 $\mathrm{R}_{2}$ values obtained in the un-irradiated gel in different location (quoted uncertainties are $\pm 1 \mathrm{SD})$

3.4 $R_{2}$ values obtained at different positions (quoted uncertainties are $\pm 1 \mathrm{SD}$ )

3.5 $R_{2}$ values measured in the gel vials imaged inside the gel phantom and polystyrene box

3.6 Elemental composition (\% by weight) of dosimetry gels

3.7 Physical quantities of various substance relevant to gel dosimetry

3.8 The effect of total monomer concentration on the gel sensitivity

3.9 The effect of acrylamide concentration on the gel sensitivity

3.10 The effect of bis-acrylamide concentration on the gel sensitivity

3.11 The effect of gelatin concentration on the gel sensitivity

3.12 Variation of the BANG gel response with energy

3.13 $\mathrm{R}_{2}$ values obtained using a 1 and 1.5 Tesla MRI scanner

3.14 Absolute dose measurements in the irradiated gels

3.15 Sensitivity of the gel to radiation field light

3.16 Variation of gel response with multiple exposure

4.1 Comparison of the absolute doserate measured at $2 \mathrm{~cm}$ from the centre of the source for two different experiments 


\section{Chapter 1. Modern developments in radiotherapy}

\subsection{Introduction}

In the last two decades the availability of $\mathrm{x}$-ray computerised tomography (CT) and other image modalities such as magnetic resonance imaging (MRI), has meant that three-dimensional treatment planning in radiotherapy has become a reality (Lichter et al 1988, Webb 1993). The advent of these facilities, in conjunction with developments in modern radiotherapy machines, such as multileaf collimation has given rise to what is known as conformal radiotherapy, that is the possibility of shaping the irradiated region precisely to the tumour volume, sparing more of the surrounding healthy tissue. Prior to this, beam shaping in two dimensions was achieved by using customized blocks made of low melting point alloy. Conformal therapy can also be achieved in three dimensions by creating irregular beam profiles for each individual patient to give the required dose distribution e.g. using customized compensation filters (Landberg 1995) or multileaf treatments (Webb 1998). This process is known as intensity modulated conformal radiotherapy. The development of such radiotherapy techniques can make treatment planning quite complex and treatment delivery more prone to error. Today with planning systems using CT and MRI data, together with developments in three-dimensional planning, more accurate treatment plans can be produced for both conventional and conformal therapy (Sharrock and Read 1998). The current status of 3-D conformal therapy and treatment planing have been reviewed in detail in the literature (Webb 1993). 


\section{$1.2 C T$ in radiotherapy planning}

The potential advantages of x-ray CT scanning for radiotherapy were recognised soon after its availability. Before CT, a pair of planar radiographs were used to determine target volume. However the extent of the tumour was often not clearly shown, information on the three dimensional shape of the tumour and location of sensitive organs was not provided and the determination of the body contour was difficult (Webb 1993). The ability of the CT scan to provide improved information on the extent of the tumour, its location and size together with information on normal tissue overcame many of the limitations associated with the planar radiograph. In recent years, the availability of CT scans for radiotherapy treatment planning has

resulted in the introduction of CT-based computer planning systems, capable of utilising the CT data. Since several CT slices are taken of the treatment volume the dose distributions can be calculated and displayed on each slice, and the treatment plan adjusted to give the optimum treatment for the volume involved.

The x-ray CT scan can supply quantitative information about tissue inhomogeneities (Geise and McCullough 1997, Kijewski and Bjärngard 1978) and this makes it possible to calculate the radiation dose distribution in the patient taking into account density differences in the irradiated volume. This can be done by using the CT numbers which correspond to the attenuation coefficients of tissue for diagnostic beam energies. Several works (e.g. Parker et al 1979, Cassell et al 1981 and Edwards et al 1981) investigated the incorporation of the CT number directly into the treatment planning program in order to make inhomogeneity corrections.

\subsection{MRI in radiotherapy planning}

Unlike the CT image, which is sometimes unable to clearly differentiate between tumour and normal tissue, MRI provides excellent soft tissue contrast (Gibbs et al 1997). In addition MRI can produce images in three planes, coronal, sagittal and 
axial, which allows more accurate determination of tumour volume and precise delineation of the normal tissue at risk (Coffey et al 1984). The superior anatomical information which is not provided by CT has led to growing interest in the use of MRI images in treatment planning. However the data from MRI images do not supply information about the electron density that is required for inhomogeneity corrections in dose calculations. The non-uniformity of the magnetic field can also cause geometrical distortion of the images (Lichter et al 1988 and Kessler et al 1991). The best use of the information provided by MRI is obtained when the images are integrated with $\mathrm{CT}$ data to optimize radiotherapy treatment planning. The methods by which MRI data can be transferred and integrated into a CT-based planning system have been described by Kessler et al (1991).

\subsection{Dosimetry for modern radiotherapy}

The complexity of dose delivery using novel techniques necessitates both the verification of the planned dose distribution in a tissue equivalent phantom simulating the treatment condition, to increase confidence in the technique, and the requirement to determine the absorbed dose distribution in three-dimensions. This could be achieved using a dosimeter that has a number of important characteristics. The desirable features for a dosimeter for conformal therapy are as follows:

1) Reproducibility of the response

2) Stability before and after irradiation

3) Independence from doserate, temperature and radiation quality

4) Linear dose response

5) Simple preparation and analysis

6) Ability to measure 3-D dose distribution with high spatial resolution

7) Tissue equivalent 
Each of the conventional dosimetry methods such as ion chambers, thermoluminescent dosimetry (TLD), silicon diodes and radiographic film has its own limitations. Dosimetry with ion chambers in a water tank can result in relatively poor resolution because of the measurement of a limited number of points or profiles and the construction of an accurate dose distribution is very time consuming. TLD dosimetry is also time consuming and requires the transfer of the detectors from phantom to a readout device and long measurement times if a sufficiently large number of detectors are used. Film dosimetry gives better resolution and can be used to measure the dose in a multi plane film phantom (Low and Mutic 1997). However construction of such a phantom is difficult and time consuming and also there is still some concern about the uncertainties in film processing and calibration (Cheng and Das 1996). Recently there has been an increasing interest in gel based chemical dosimetry in which chemical changes due to irradiation alter the relaxation rate of water protons which can be measured using MRI.

It is well known that most effects of ionising radiation whether they are physical or chemical, can be used as a means of measuring the absorbed dose of radiation. Therefore, in addition to measuring ionisation in gas, which is the principal method of measuring absorbed doses of $\mathrm{x}$ or gamma rays, other radiation effects may also be used. In chemical dosimetry for example, the radiation dose is determined by measuring the chemical change produced. Aqueous solutions are the most widely used chemical dosimeters in radiation dosimetry.

One of the major problems in determining the absorbed dose accurately is the fact that the dosimeter may perturb the radiation field in the material in which the absorbed dose is to be determined. Therefore, it is also desirable to have a dosimeter that does not differ significantly from the medium in question. Chemical dosimeters are useful since they offer a very broad range of densities and atomic compositions. Before discussing the role of gel based MRI dosimetry in radiotherapy measurements, one of the more widely used chemical dosimeters, Fricke dosimetry, is considered briefly. 


\subsubsection{Fricke dosimetry}

Fricke dosimetry has been in use as a radiation dosimetry system for many years. It is based on the oxidation of ferrous ions to ferric ions as an aerated acid solution of ferrous sulphate is irradiated. The absorbed dose can then be estimated by measuring the concentration of ferric ions before and after irradiation. The simplified reactions in which ferrous ions oxidize to ferric ions are:

$$
\begin{aligned}
& \mathrm{H}_{2} \mathrm{O} \rightarrow \mathrm{H}_{2} \mathrm{O}^{+}+\mathrm{e}^{-}+\mathrm{H}_{2} \mathrm{O}^{*} \\
& \text { (radiation) } \\
& \qquad \mathrm{H}_{2} \mathrm{O}^{*} \rightarrow \mathrm{H}+\mathrm{OH}
\end{aligned}
$$

where $\mathrm{e}^{-}$is an ejected electron, $\mathrm{H}_{2} \mathrm{O}^{*}$ is an excited water molecule and $\mathrm{OH}$ is the hydroxyl radical

A ferrous ion is oxidised to a ferric ion by the hydroxyl radical

$$
\mathrm{Fe}^{2+}+\mathrm{OH} \rightarrow \mathrm{Fe}^{3+}+\mathrm{OH}^{-}
$$

The hydrogen atom (1.2) reacts with dissolved oxygen to give a hydroperoxy radical $\mathrm{HO}_{2}$

$$
\mathrm{H}+\mathrm{O}_{2} \rightarrow \mathrm{HO}_{2}
$$

The hydroperoxy radical then oxidizes a ferrous ion

$$
\mathrm{Fe}^{2+}+\mathrm{HO}_{2} \rightarrow \mathrm{Fe}^{3+}+\mathrm{HO}_{2}^{-}
$$

the $\mathrm{HO}_{2}{ }^{-}$then reacts to form hydrogen peroxide: 


$$
\mathrm{HO}_{2}^{-}+\mathrm{H}^{+} \rightarrow \mathrm{H}_{2} \mathrm{O}_{2}
$$

which then forms a further ferric ion

$$
\mathrm{Fe}^{2+}+\mathrm{H}_{2} \mathrm{O}_{2} \rightarrow \mathrm{Fe}^{3+}+\mathrm{OH}+\mathrm{OH}^{-}
$$

The standard solution for the Fricke dosimeter is prepared from triply distilled water, or high purity water and contains ferrous sulphate $\left(0.001 \mathrm{M} \mathrm{FeSO}_{4}\right)$ or ammonium ferrous sulphate $\left(\mathrm{Fe}\left(\mathrm{NH}_{4}\right)_{2}\left(\mathrm{SO}_{4}\right)_{2}\right)$, sulphuric acid $\left(0.8 \mathrm{~N} \mathrm{H}_{2} \mathrm{SO}_{4}\right)$ and is saturated with air ( Fricke \& Hart 1972). Since chloride ions can desensitise the system to organic impurities, $\mathrm{NaCl}$ is added to the solution in order to inhibit the oxidation of ferrous ions by organic impurities (Dewhurst 1951). The modified Fricke solution therefore contains $0.001 \mathrm{M}$ of $\mathrm{NaCl}$.

Conventionally the method that is most commonly used to measure the formation of ferric ions is spectrophotometric analysis (Spinks \& Woods 1964). This analysis compares the optical density of the non-irradiated and irradiated dosimeter solution using a wavelength of about $3040 \AA$. This wavelength is chosen because of the light absorption properties of the ferric ion. The radiation chemical yield $\left(\mathrm{Fe}^{3+}\right)$ is usually expressed in terms of the $G$ value which is defined as the number of molecules or ions $\left(\mathrm{Fe}^{3+}\right)$ liberated per $100 \mathrm{eV}$ of absorbed energy. The changes in concentration of ferric ions in dosimetric solution can be also detected using nuclear magnetic resonance (NMR) (Gore et al 1984a,b). This is discussed in detail in the next section.

\subsection{MRI In Radiotherapy Dosimetry}

\subsubsection{Fricke gel dosimetry}

Both ferric and ferrous ions are paramagnetic and therefore both increase the spin relaxation rates of the water protons of the aqueous Fricke solution but to a 
different extent. Based on this fact, the change in concentration of ferric ions in the Fricke solution can be measured using NMR. Given the same concentration of ferrous and ferric ions, the magnitude of increase in the longitudinal relaxation rate by ferric ions is more than 19 times that for ferrous ions (Gore et al 1984a,b) and the spin-spin relaxation rate is 26.7 times greater than that for ferrous ions (Luciani et al, 1996).

The basic principle that governs the proton NMR relaxation behaviour of irradiated aqueous Fricke solution is well established. Protons in water molecules in the $\mathrm{Fe}^{2+}$ and $\mathrm{Fe}^{3+}$ co-ordination shells experience enhanced relaxation while the other protons behave like those in pure water (Gore et al 1984a, Podgorsak 1992). Since ferrous and ferric ions are paramagnetic they can reduce proton relaxation times. Even in small concentrations the irradiated Fricke solutions should have a dose dependent increase in the NMR relaxation rates. A linear increase in the spin lattice and the spinspin relaxation rate was observed by Gore et al (1984a).

Since the proposal in 1984 by Gore and co-workers that NMR relaxometry of irradiated Fricke dosimeters could be used to measure absorbed dose, there has been a number of reports of MR imaged dose distributions of phantoms irradiated by X-rays, high energy X-rays and electron beams. These studies confirm that NMR can measure the radiation induced conversion of ferrous to ferric ions very well (Olsson et al, 1989).

In radiotherapy treatment planning, however, not only is a knowledge of absorbed dose required but also a knowledge of the spatial dose distribution in the patient. A method of measuring spatial dose distribution using the Fricke dosimeter and NMR was proposed by Gore et al in 1984. The method measures absorbed dose distributions in a tissue equivalent medium (gel) doped with Fricke solution which is interrogated using magnetic resonance imaging (MRI). The distribution of $\mathrm{Fe}^{3+}$ is fixed by the gel, therefore it is possible to measure a 3D absorbed dose distribution in the gel using MRI which is of great interest in the field of radiotherapy (Day 1990).

Different types of gel have been used as a tissue equivalent medium, including gelatin (Olsson et al 1989, Hazle et al 1991, Prasad et al 1991 Duzenli et al 1994), 
agarose (Appleby et al 1987,1988, deGuzman et al 1989, Olsson et al 1989, Kron \& Pope 1994), Sephadex G-200 combined with Sumikagel N-100 (Hiraoka et al 1993) and polyacrylamide (deGuzman et al 1989). Table 1.1 lists the typical concentrations that have been used.

\begin{tabular}{|c|c|c|}
\hline Gel & Concentration & Reference \\
\hline Gelatin & $1-12 \%$ & $\begin{array}{r}\text { Duzenli et al 1994, Hazle et al 1991, Olsson } \\
\text { et al 1989, Prasad et al 1991 }\end{array}$ \\
\hline Agarose & $0.5-2 \%$ & $\begin{array}{c}\text { Appleby et al 1987,1988, deGuzman et al } \\
1989, \text { Olsson et al 1989, Kron \& Pope 1994 }\end{array}$ \\
\hline $\begin{array}{c}\text { Sephadex G-200+ } \\
\text { Sumikagel N-100 }\end{array}$ & $1 \%$ & Hiraoka et al 1993 \\
\hline Polyacrylamide & $5 \%$ & deGuzman et al 1989 \\
\hline
\end{tabular}

Table 1.1. Typical gel concentration of the NMR Fricke dosimeter

Appleby et al (1988) indicated that both agarose and gelatin systems increase the ferric ion yield ( $\mathrm{G}$ value) in the gel. The sensitivity of the Fricke gel system to radiation is higher than the corresponding aqueous Fricke solution by a factor of 4 when agarose gel is used (Olsson et al 1989) and by a factor of 2.2 when gelatin is used (Olsson et al (1989) and Prasad et al (1991). Therefore adding gelling agent to the dosimeter solution does not only have the advantage of the fixing dose distribution within the system but also provides a gain in sensitivity.

A number of workers have investigated the NMR properties of Fricke gel systems. Chan and Ayyangar (1993) investigated the water equivalency of Fricke gel for different photon and electron beam energies using Monte Carlo simulations. Chan and Ayyangar (1993) investigated the water equivalency of Fricke gel for different photon and electron beam energies using Monte Carlo simulations. They found the 
gel is water equivalent as the differences in calculated density, central axis depth dose and isodose distributions were within less than $1 \%$.

The effects of ferrous ion and sulphuric acid concentration on the sensitivity of the Fricke gel system were investigated by Olsson et al (1991). They found that the sensitivity of the system decreased with increasing ferrous ion concentration and that the increase in sensitivity is rather slow with increasing sulphuric acid concentration.

Another aspect of interest for a dosimeter is the dependence on doserate. Schulz et al (1990) examined the doserate response of the dosimeter gel using a 25 MV X-ray beam up to $24.2 \mathrm{~Gy} \mathrm{~min}^{-1}$. They found no dependence on absorbed doserate. Appleby et al (1988) obtained similar results for doserate from 0.43 to 3.74 Gy $\mathrm{min}^{-1}$ and Olsson et al (1989) from 1.3 to $4.1 \mathrm{~Gy} \mathrm{~min}^{-1}$ using $6 \mathrm{MV} \mathrm{X}$-rays.

The dependence of the gel response on beam energy has also been investigated by Luciani et al (1996). They have found no changes in the dose response of Fricke agarose gel using $\mathrm{x}$-ray source with a beam equivalent energy of $90 \mathrm{keV}$ and $\mathrm{Co}^{60}$.

Various limits to the linearity of the dose response have been reported; up to 10 Gy (Appleby et al 1987), more recently linearity up to $20 \mathrm{~Gy}$ by measuring longitudinal relaxation rate, $R_{1}$ (Kron \& Pope 1994), up to 30 Gy measuring $R_{1}$ (Thomas et al 1992), 36 Gy measuring transverse relaxation rate, $R_{2}$ (Gambarini et al 1994) and 50 Gy measuring $R_{1}$ and oxygenating the gel (Land 1994).

In an attempt to improve the sensitivity of the system (i.e. increase the $G$ value), Prasad et al (1991) added $1.32 \mathrm{mM}$ benzoic acid instead of $1 \mathrm{mM}$ sodium chloride to the system. Their results showed an enhancement of about 4.5 in the measurement of the transverse relaxation rate and about 2.5 in the measurement of the longitudinal relaxation rate. In contrast to Prasad et al (1991), Gambarini et al (1994) found no significant enhancement by adding $1.5 \mathrm{mM}$ and $3.5 \mathrm{mM}$ benzoic acid with and without $\mathrm{NaCl}$.

The reproducibility of the dose response was found to be within $\pm 7 \%$ in the same batch of samples and up to $50 \%$ between different batches of samples (Kron and Pope 1994). The work carried out by Luciani et al (1996) pointed to $20 \%$ variation in 
$\mathrm{T}_{1}$ and up to $10 \%$ in $\mathrm{T}_{2}$ measurement using the same batch of agarose and keeping the preparation constant. Gambarini and his colleagues (1994) have reported good reproducibility for different preparations of the dosimeter provided that the agarose and the constituent chemicals come from the same industrial batches.

Comparison of central axis depth dose measurements were made using the Fricke gel system and an ionisation chamber in electron and photon beams of different energies (Smajo 1996). The measurements resulted in a good agreement for electrons beyond the depth of dose maximum but discrepancies for photons.

No significant diffusion of the ferric ions in the gel was found for up to 10 hours after irradiation by Prasad et al (1991). In contrast, investigations carried out by Schulz et al (1990) showed a diffusion coefficient of $1.58 \pm 0.11 \mathrm{~mm}^{2} \mathrm{~h}^{-1}$ for a concentration of $25 \mathrm{mM}$, and $1.83 \pm 0.14 \mathrm{~mm}^{2} \mathrm{~h}^{-1}$ for a concentration of $12.5 \mathrm{mM}$ Sulphuric acid. The diffusion coefficient of ferric ions has also been found to be $1.9 \pm$ $0.95 \mathrm{~mm}^{2} \mathrm{~h}^{-1}$ by Olsson et al (1992) using $1.5 \%$ agarose and $50 \mathrm{mM}$ Sulphuric acid. Spontaneous oxidation can also occur in the Fricke gel dosimeter. This decreases the spin lattice relaxation time by about 4 and $2 \mathrm{~ms} \mathrm{~h}^{-1}$ for gelatin and agarose, respectively, during the first $40 \mathrm{~h}$ of the preparation as reported by Olsson et al (1989). The data obtained by Schulz et al (1990) also indicated that $3 \%$ of the ferrous ions were oxidised during the process of the gel preparation. In addition Duzenli et al (1994) highlighted that even the spontaneous oxidation of ferric ions increases the relaxation rate by up to $4 \%$ per day depending on the concentration of the gelatin in the system but does not significantly change the shape of the dose response curve. Therefore it is generally suggested that a shorter time delay between preparation, irradiation and MR imaging is desirable. For example, by reducing the time delay between the gel irradiation and MR scanning to less than five minutes and using high concentration of the gel matrix, $7.5 \%$, Chan and Ayyangar (1995) found that the diffusion of ferric ions is negligible.

To reduce the diffusion of ferric ions, Rae et al (1996) investigated the effect of chelating agent in the Fricke gel. They found adding xylenol orange to the gel reduces 
the diffusion rate from $0.82 \mathrm{~mm}^{2} \mathrm{~h}^{-1}$ to $0.44 \mathrm{~mm}^{2} \mathrm{~h}^{-1}$ but at the expense of reduction in gel sensitivity from 0.0129 to $0.0093 \mathrm{~s}^{-1} \mathrm{~Gy}^{-1}$.

Although the application of Fricke gel dosimetry for the measurement of absorbed dose distribution has so far mainly concentrated on external radiation beam, some work has also been done on brachytherapy. The suitability of the Fricke gel dosimeter in both high doserate (HDR) brachytherapy (Olsen \& Hellesnes 1994, Schreiner et al 1994) and low doserate (LDR) brachytherapy (Olsen \& Hellesnes 1994) has been investigated. The results indicated that gel Fricke dosimetry is a reliable means of measuring dose distribution in brachytherapy. In LDR brachytherapy the use of the gel dosimetry for longer irradiation times may be limited because of the diffusion of ferric ions.

Schulz et al (1993) used Fricke gel to assess the accuracy of the localisation of the target in stereotactic radiosurgery by $\mathrm{CT}$ and MRI. The results confirmed that the Fricke system can provide a practical method to check the accuracy of stereotactic radiosurgery procedures. The verification of dose distribution in radiosurgery has also been made using Fricke agarose gel by Rousseau et al (1994) and comparison has been made with calculations. They found good agreement in dose distribution between the gel and calculation with an accuracy of $1 \mathrm{~mm}$.

In addition the suitability of Fricke gel dosimetry for measurements of a mixed field of neutrons and gamma rays has been reported (Cantone et al 1992); and measurements of the distributions of photon fields using dynamic wedge have also been studied (Bengtsson et al 1996).

\subsubsection{Choice of dosimeter}

In NMR gel dosimetry, unlike other methods of dosimetry, the detector and phantom are the same and are tissue equivalent. The one exception is dosimetry using Perspex (Orton 1966), though Perspex is only approximately tissue equivalent. There 
is no need, therefore to place a dosimeter into the phantom and no need to remove part of the irradiated material for measurement. In addition absorbed dose can be measured in any plane and there is no need for correction factors to be applied for perturbation of the radiation field. Moreover the gel can be shaped to be part of an anthropomorphic phantom which can be irradiated and the dose distribution measured with high spatial resolution in any plane of interest. An NMR dosimetry system also has the potential to measure absorbed dose distributions from complex multiple fields such as those used in conformal therapy (Day 1990, Bucciolini et al 1991, Hefner et al 1992, Thomas et al 1992, Kron et al 1993).

Another use of the gel as a phantom is for calibrating the MRI scanner . A variety of materials have been used as a tissue equivalent material for this purpose. They include aqueous solutions of copper, manganese or nickel ions and agarose gels. Although these materials are useful, they are not exactly tissue equivalent because their spin-lattice $\left(T_{1}\right)$ relaxation times and spin-spin relaxation times $\left(T_{2}\right)$ differ from those of tissue (Howe 1988, Mitchell et al 1986). Therefore a combination of paramagnetic ions and a gel has been developed that can be adjusted to produce a wide range of $T_{1}$ and $T_{2}$ similar to those of biological tissues. In these materials the $T_{1}$ values are mainly controlled by concentration of the paramagnetic ions and $T_{2}$ values by the gel concentration (Walker et al 1988).

In the case of the Fricke gel, there is a major limitation on its use. The ferric ions tend to diffuse significantly through the gel and consequently blur the MRI image after radiation (Schulz et al 1990, Balcom et al 1995). Diffusion of ferric ions, with relatively low sensitivity and poor reproducibility (Day 1990, Hazel et al 1991, Podgorsak and Schreiner 1992) are the factors that limit the possibility of quantifying the radiation dose and demand more investigation. 


\subsubsection{Polymer gel Dosimetry}

In 1993 Maryanski and co-workers introduced an alternative to the Fricke gel, a 'polymer gel' for imaging dose distributions in three dimensions using MRI. This type of dosimeter is based on polymerization and cross-linking of acrylic monomers in an aqueous gel by the action of radiation. The effect of this process on the MRI measurements is similar to the Fricke gel dosimeter in which paramagnetic ions (ferrous and ferric) increase the spin relaxation rates of the water protons: radiationinduced polymerization plays the same role with proton relaxation rates in water but with some important advantages, these are:

1) In the Fricke gel, ferric ions tend to diffuse through the gel before and after irradiation at a rate of $1.83 \times 10^{-2} \mathrm{~mm}^{2} \mathrm{~h}^{-1}$ in a $1.0 \%$ agarose gel, which leads to blurring of the dose distribution, and a consequent loss of resolution (Schulz et al 1990). In the polymer gel the polymer produced is relatively immobile and its image after irradiation is reported to be stable, this means that the dose distribution will not change with time (Maryanski et al 1993). Therefore it is possible that the polymer gel can be imaged after irradiation at a time which is more convenient and the calculation of relaxation rate can be made with more accuracy.

2) The sensitivity of polymer gels to radiation is greater than the Fricke gels, which is especially useful in high dose gradients (Hazel et al 1991, Maryanski et al 1994).

3) The cross-linked polymer is not water soluble, and precipitates from the aqueous phase of the transparent gel, therefore the polymerization area can be easily seen. The opacity of the polymerized area increases as the radiation dose increases, thus the gel may be measured quantitatively by an optical densitometer and the potential exists for production of dose distributions using optical methods (Maryanski et al 1994, 1996b).

Both agarose and gelatin have been used as a gelling agent. Two types of the gel have been reported in the literature, BANANA (1993) and BANG (1994). 
Agarose was used as gel material in the former and gelatin in the latter. The acronym BANANA stands for Bis, Acrylamide, Nitrous oxide And Agarose. Oxygen inhibits the polymerization process, therefore nitrous oxide $\left(\mathrm{N}_{2} \mathrm{O}\right)$ was used to remove oxygen

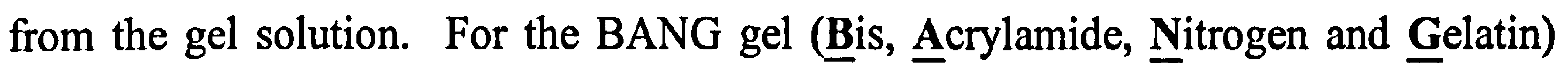
gelatin and nitrogen were use as a gel material and deoxygenating gas.

Recently BANG-2 was introduced by Maryanski et al (1996a). This is claimed to be an improved formulation of the polymer gel and differs from the BANG gel in the substitution of acrylic acid for acrylamide. Introduction of the polymer gel dosimetry and its potential advantages over Fricke gel has given rise to several initial investigations of the properties of the gel.

The linear response of the BANANA polymer gel to radiation was reported up to $15 \mathrm{~Gy}$ by Maryanski et al (1993) and up to $12 \mathrm{~Gy}$ by Djennaui (1994) both measuring $R_{1}$. The response for BANG gel has been shown to be linear up to $8 \mathrm{~Gy}$ by Maryanski et al (1994) measuring $R_{2}$. The slope of the dose response curve increases with increasing NMR frequency when measuring $R_{2}$. In contrast, the slope of $R_{1}$ versus dose decreases with frequency (Maryanski et al 1993).

It has been reported that at a dose of $5 \mathrm{~Gy}$ the polymerization becomes visible (Maryanski et al 1993 and Djennaui 1994). The opacity of the irradiated gels increases with radiation dose.

The effect of temperature on the dose response of the BANG gel was investigated by Maryanski et al (1995). They reported that whereas the gel temperature at the time of the irradiation had no influence on the shape of the dose response curve, the gel temperature at the time of imaging has a significant effect. The slope decreases from $0.52 \mathrm{~s}^{-1} \mathrm{~Gy}^{-1}$ at $5^{\circ} \mathrm{C}^{\circ} 0.15 \mathrm{~s}^{-1} \mathrm{~Gy}^{-1}$ at $40^{\circ} \mathrm{C}$, more than 0.01 $\mathrm{s}^{-1} \mathrm{~Gy}^{-1}$ decrease per degree Centigrade.

The dependence of the percentage concentration of the cross linker N,N'Methylene-bisacrylamidee (bis) per total amount of monomer on dose sensitivity has been investigated (Maryanski et al 1997a). The results indicated that dose sensitivity 
is strongly affected by the fraction of bis with a maximum sensitivity at about $50 \%$ by weight.

The improvements in gel dosimetry offered by the "polymer gel" open up some important potential applications in radiotherapy physics. Maryanski et al (1996a) used BANG-2 gel to confirm the dose distributions for both stereotactic radiosurgery and high doserate (HDR) brachytherapy. They found good agreement between the dose distribution measured in the gel and the one calculated using the treatment planning system. Martin et al (1997) used BANG gel to characterise an Ir-192 HDR brachytherapy source. The measured doserate constant agreed with the result measured using TLD and radiochromatic film. Dose distributions for endovascular brachytherapy were studied by Maryanski et al (1997b) using BANG polymer gel. They recorded the dose distribution around a beta-emitting rhenium source with spatial resolution of 120 micrometers. Verification of dose distribution from interstitial radiosurgery and also external beam breast therapy were made by Baldock et al $(1996 \mathrm{a}, \mathrm{b})$ using BANG gel. Their results also indicated a good agreement between the measurement using the gel and the treatment plan. Current work in progress in polymer gel includes comparison between BANG and ferrous-agaroseXylenol orange gels (Wong et al 1995), confirmation of the accuracy of repeat-fixation in stereotactic radiation therapy (Ibbott et al 1996), 3D conformal therapy (Audet et al 1996), (Baustert et al 1997) and applications in proton beam dosimetry (Duzenli et al 1997).

More recently an optical system for measuring dose distributions in polymer gels has been developed (Gore et al 1996). This measures three-dimensional dose distribution by scanning of the polymer gel (Maryanski et al 1996b) with a laser beam. The optical tomographic technique is based on the same principles that govern $\mathrm{x}$-ray computed tomography. Dose distributions are reconstructed from changes in optical density of the irradiated polymer gel by series of projections taken from different angles. The optical scanning system is in the early stage of its development. The aim 
is to provide a system that can perform as many functions as currently are performed by MRI.

The results of investigations using optical scanning of irradiated polymer gels not only confirm that the shape of the dose response curve depends on the concentration of cross-linking monomer but also depends on the wavelength of light in the system. The results have proved however the ability of polymer gels to provide an optical recording medium for radiation dosimetry.

\subsection{Aims of the investigation}

The present investigation has the following aims:

(1) To measure the main properties of the BANG polymer gel, in particular those which have not been reported or reported in part or need quantification. These include energy response, composition, time after radiation, diffusion, gel calibration, effect of different batch and effect of RF inhomogeneity.

(2) To investigate the practical and technical limitations of BANG gel as a dosimeter as well as the problems associated with the MRI imaging.

(3) To evaluate the usefulness of the polymer gel in different areas of radiotherapy including the evaluation of a 3-D planning system, the confirmation of compensator based conformal therapy, and brachytherapy.

(4) To investigate possible novel applications of gel dosimetry particularly in boron neutron capture therapy or measurements in areas that present difficulties using conventional methods. 


\section{Chapter 2. Basic theory of MRI}

\subsection{Introduction}

Nuclear Magnetic Resonance (NMR) involves the energy levels of nuclei when they are placed in a magnetic field. Transitions between levels can occur following the absorption of energy at radio-frequencies. The first successful NMR experiments were carried out in 1946 by Bloch and Purcell which gained them the Nobel Prize in 1952. Since then, the discovery of chemical shift and development of NMR spectroscopy have made it a powerful analytical tool for studying the structure and concentration of molecules rather than just being a method of measuring the magnetic properties of atomic nuclei. Up until 1973, the application of NMR techniques were confined to the determination of the molecular structure of pure homogeneous samples. The major biomedical and medical interest in the technique arose from the possibilities of making non-invasive measurements in living tissue. Damadian in 1972 and Lauterbur in 1973 indicated that the human body could be imaged for clinical purposes by NMR. Since then there has been very rapid development in the number and range of techniques, with the first clinical trials of medical imaging taking place in 1980 (Mansfield and Morris, 1982). In medical NMR imaging or Magnetic Resonance Imaging (MRI) data are collected and processed to produce a cross-sectional image of the spatial distribution of selected nuclei at any anatomic level.

Unlike traditional clinical radiography where the production of images is based on the interaction of $\mathrm{x}$-rays with electrons surrounding atoms, all MRI systems measure the signal that is induced following a pulse of radio-frequency (RF) radiation by the magnetic field of protons in the atoms of body tissues. In order to obtain a useful image of a whole body cross section, the nuclei concerned must be relatively abundant and must behave suitably in a magnetic field (Gore, 1983). 


\subsection{The principles of MRI}

The principle of MRI is based on the fact that the positively charged nucleus of the hydrogen atom, the proton, possesses a spin. The spinning charge makes protons behave like a small bar magnet. In the absence of an applied magnetic field the protons are randomly oriented. When a large number of such magnetic nuclei are placed in a large static external magnetic field they align themselves with this field. Some of the protons align in the direction of the magnetic field (parallel to it), and the other align in the opposite direction (anti-parallel to it), corresponding to two energy levels. Normally the number of protons aligned parallel to the magnetic field is slightly more than those that align anti-parallel, since the former has the lower energy level. Given 2 million protons, 1,000,005 will be in the lower energy level while the remaining 999,995 will be in the higher energy level (Roth 1984). This results in the production of net magnetisation $(\mathrm{M})$ in the direction of the field (Figure 2.1). In addition the protons precess about the external magnetic field.
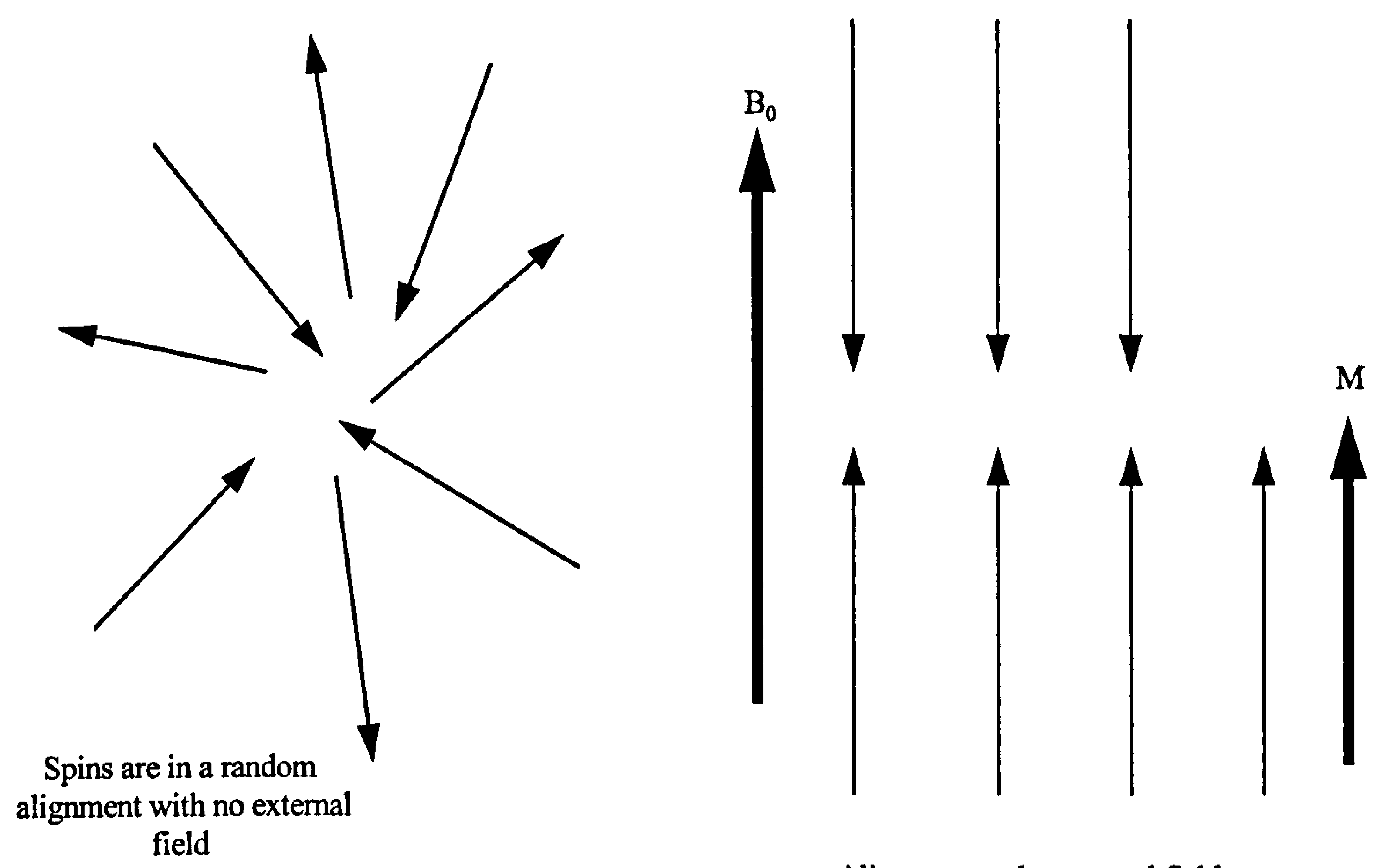

Alignment to the external field

Figure 2.1. The net magnetization in the direction of the external field 
If radio-frequency radiation of the right energy is applied to protons they absorb energy and move in step in the same direction at the same time. This in-phase movement of the protons will induce a voltage in the RF coil. After the radio frequency pulse is switched off the protons lose their coherent movement gradually and the induced signal decays with time. The intensity of the signal depends on the number of protons that have been magnetised which in turn depends on the strength of the applied magnetic field. As the strength of the external magnetic field increases the ratio of the number of parallel to anti parallel protons also increases, which results in stronger net magnetization and consequently higher signal intensity.

\subsection{Physical basis}

\subsubsection{MR sensitive nuclei}

Not all atomic nuclei can be studied with magnetic resonance. In a nucleus, the total angular momenta of the constituent nucleons add vectorially to make the nuclear spin. Nuclei in which protons and neutrons separately fully occupy a set of single-particle energy levels, will have nuclear spin zero since the angular momenta of the nucleons cancel each other out. Only two neutrons or two protons can occupy a level with the same energy, therefore the spin of the ground state of all nuclei with an even number of protons and an even number of neutrons is zero. Any nucleus with an odd number of protons or neutrons or protons and neutrons will have a net magnetic spin and can interact with an external magnetic field (Williams 1991). Hydrogen atoms which contain a single proton are the most favoured nuclei for MRI due to their high concentration in tissue, and high gyro magnetic ratio $(\gamma)$. The gyro magnetic ratio is defined as the ratio of the magnetic moment to the angular momentum.

When there is no magnetic field, protons may take any orientation due to their thermal motion. Magnetic moments from the nuclei cancel out each other therefore no net magnetisation is generated. In the presence of an external magnetic field they align themselves with this magnetic field. As there are slightly more nuclei aligned 
parallel due to the lower energy level, there is always a small excess in this direction that produces a net magnetisation in the direction of the applied magnetic field.

\subsubsection{Precession}

When hydrogen atoms are placed in an external magnetic field, the interaction of their inherent spin angular momentum with the external magnetic field produces an additional rotation of the magnetic moment around it. This type of movement is called precession and causes the magnetic moments to follow a circular path around the magnetic field (Figure 2.2).

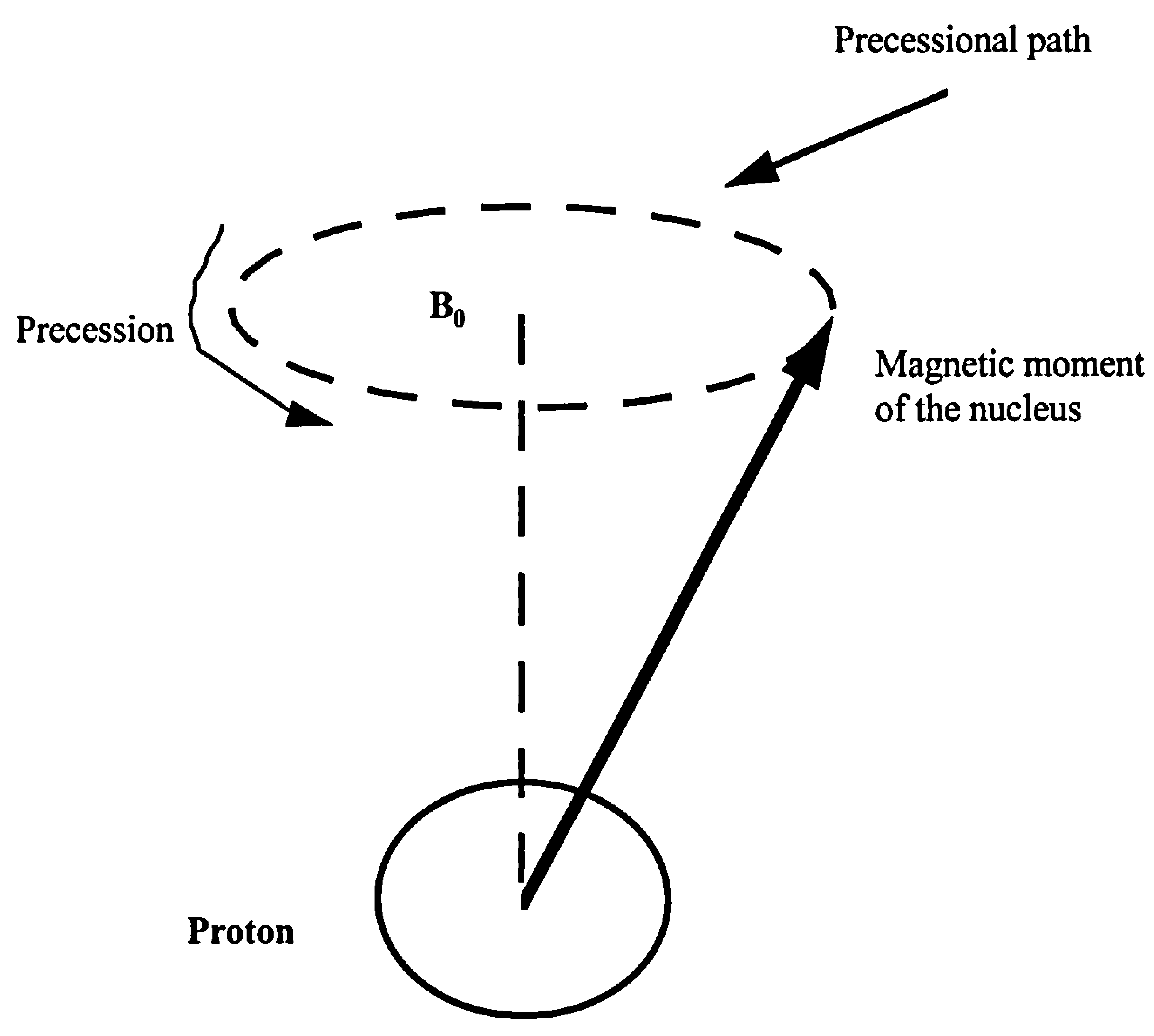

Figure 2.2. Precession

The rate at which the magnetic moments rotate around the external magnetic field is called the precessional frequency. The precession frequency for each nuclei depends on the magnetic field strength and is unique for each nucleus at that field 
strength. The stronger the magnetic field the higher the precession frequency. The value of the precessional frequency is derived from the Larmor equation

$$
\omega=\gamma \mathrm{B}_{0}
$$

where

$\omega$ is the precessional or Larmor frequency.

$\gamma$ is the gyro magnetic ratio which characterises the type of nuclei and is

unique a for particular nucleus.

$B_{0}$ is the magnetic field strength.

\subsubsection{Resonance}

Resonance is a phenomenon whereby an object is exposed to an oscillating perturbation that has a frequency close to its own natural frequency of oscillation. When a nucleus is exposed to an external perturbation in the horizontal plane at frequency corresponding to its precessional frequency, the nucleus resonates and gains energy from the external force. Therefore the net magnetization vector will move off from the direction of the static field, which is assumed to be in the vertical or $\mathrm{z}$ coordinate direction (Figure 2.3).

As a result of resonance the protons start to precess in phase in the transverse plane. Therefore as the protons precess at the Larmor frequency in the transverse plane, a voltage is induced in a receiver coil. This voltage constitutes the MR signal. The excitation magnetic field has a frequency in the megahertz or radio frequency (RF) range of the electromagnetic spectrum. In most NMR experiments the excitation magnetic field is applied in a pulsed mode (Loeffler 1990), and is generally called the RF pulse. The angle $\alpha$, that the net magnetization moves from the $z$ direction is called the flip angle. The magnitude of the flip angle depends on the strength and duration of RF pulse (Kean and Smith 1986). If the length of the pulse is such that the net magnetization tilts exactly into the xy plane, then a $90^{\circ}$ pulse has been applied. By 
doubling the pulse length to give a " $180^{\circ}$ pulse", the net magnetization can be turned completely over so that it is directed opposite to the main field.
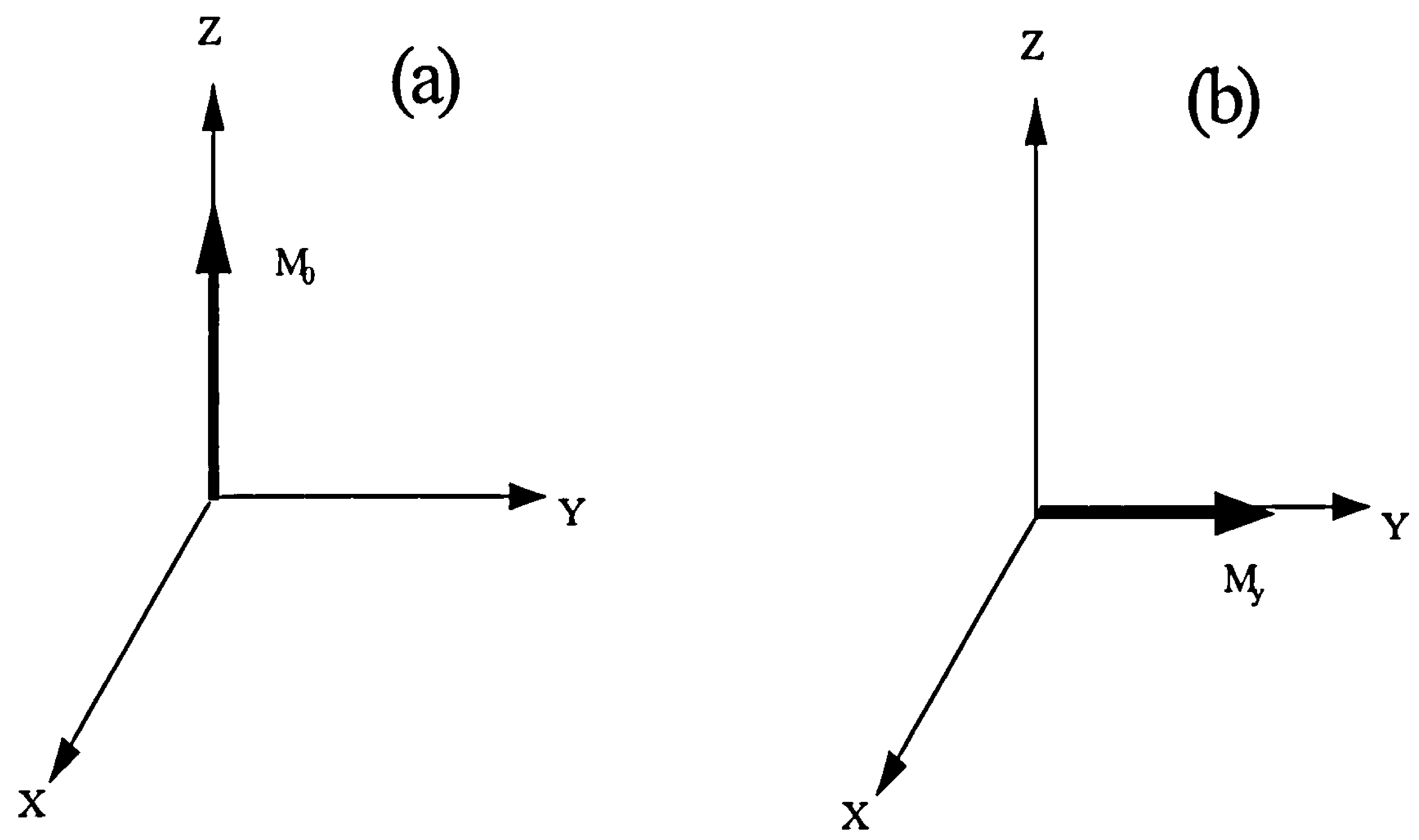

Figure 2.3. Magnetization (a) at equilibrium,

(b) after a $90^{\circ}$ pulse

The magnetic moment $M$ of a nucleus at an angle $\alpha$ to the vertical can be represented by its three components, $M_{z}, M_{y}$ and $M_{x}$. At equilibrium, $M$ is directed parallel to $B_{0}$ along $z$ so we have $M_{z}=M_{0}$ and $M_{y}=M_{x}=0$

\subsubsection{Relaxation}

When the system of protons is placed in a strong magnetic field $B_{0}$ the number of spins aligned with the field are similar to the number which are opposed to the field but with a small number extra in the direction of field and therefore a net magnetization $\mathrm{M}_{0}$ will result in the field direction $z$. If the $R F$ radiation is applied perpendicular to the $\mathrm{B}_{0}$ at proper frequency then nuclei can absorb energy and move to 
the higher energy state. The slight initial excess of nuclei in the lower energy state is the only reason that an NMR signal can be seen at all (Taylor et al 1988).

After the radio frequency pulse has been switched off, the magnetization, $M$, returns to its original alignment parallel to $\mathrm{B}_{0}$. The process by which the magnetization loses the absorbed energy and goes back to its original state is called relaxation, and the time that it takes is called the relaxation time. Relaxation time is a characteristic of particular nuclei and their chemical environment (Vennart 1985). NMR relaxation is induced by interaction of the nuclei with fluctuations in the local magnetic field. As relaxation proceeds, the magnetization decays to an equilibrium value of zero in the so called y direction (Transverse relaxation) while it increases from zero to $\mathrm{M}_{0}$ in the $\mathrm{z}$ direction (Longitudinal relaxation) (Figure. 2.4)

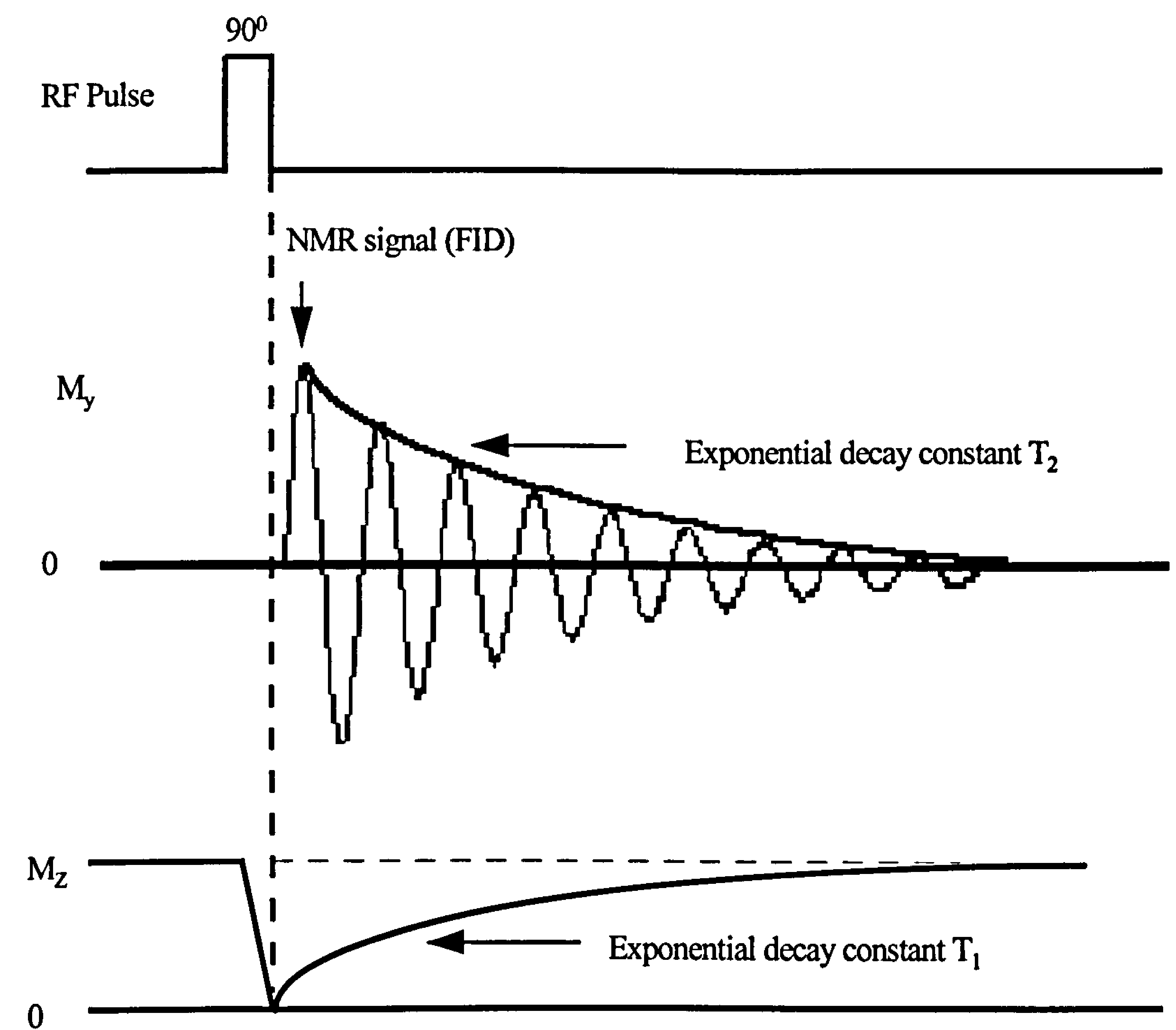

Figure 2.4. Variation in the net components of the magnetic moment with time 


\subsubsection{Longitudinal relaxation time}

The longitudinal relaxation time is often known as the $T_{1}$ or spin-lattice relaxation time. It is a measure of how rapidly the nuclear spins realign with the main external magnetic field. Longitudinal relaxation is a resonance phenomenon. If an RF pulse is sent at right angles to the $\mathrm{B}_{0}$ field and with correct frequency for the system of spins, the net magnetization will rotate into the $y$ direction and spins will precess in phase. The process of re-growth of magnetization aligned with $B_{0}$ is called longitudinal relaxation. This relaxation process is associated with transfer of the energy from excited anti parallel spins to the surrounding environment or lattice. Thus energy release to the surrounding lattice causes spins to recover their longitudinal magnetisation. This is the reason that the process is not only called longitudinal relaxation but also spin-lattice relaxation.

The relaxation time $T_{1}$ describes the energy transfer and growth of longitudinal magnetisation to equilibrium. This is the time constant and the inverse $\left(\mathrm{T}_{1}^{-1}\right)$ is called the relaxation rate $R_{1}$. The different rates of $T_{1}$ are related to how rapidly the higher energy spins transfer the excess energy to the lattice. The transfer of energy is facilitated by the presence of fluctuating magnetic fields experienced by the nuclear spins at the Larmor frequency. When the nucleus experiences fluctuating fields at the Larmor frequency, energy is transferred to the lattice.

The longitudinal Bloch equation describes the motion of nuclear magnetization in the direction of the $B_{0}$ field. The motion is governed by the time constant $T_{1}$ :

$$
\mathrm{dM}_{\mathrm{z}} / \mathrm{dt}=\left(\mathrm{M}_{\mathrm{z}}-\mathrm{M}_{0}\right) / \mathrm{T}_{1}
$$

It can be shown by integration that a solution to above equation is:

$$
M_{2}=M_{0}\left[1-\exp \left(-t / T_{1}\right)\right]
$$

This solution apply for $90^{\circ}$ pulses. 


\subsubsection{Transverse relaxation time}

Transverse magnetization occurs when the spins are perturbed so that a component of the net magnetization is tilted orthogonal to the external magnetic field. Initially, the spins all precess with the same frequency in phase. The net magnetization which is the net total vector sum of many individual spins is larger when the spins all have the same phase organisation. The MR signal can be recorded after the RF pulse is terminated.

Following the RF pulse, the signal will start to decay rapidly due to the loss of the phase coherence of the individual spins in relation to one another. The loss of phase coherence results from the fact that the spins are not precessing at the same frequencies. If the spins were precessing continuously at the same frequency they would remain in phase and the signal would persist with a constant strength. However this is not what is observed (Chakeres and Schmalbrock, 1992) because each spin contributes a small additional field to its neighbours which results in a slight difference in the frequency of each individual spin relative to its neighbour and an increasing dephasing and consequently decay of the transverse magnetisation. For this reason transverse relaxation is also known as spin-spin relaxation (Figure 2.5).

The transverse relaxation time $\left(T_{2}\right)$ is the time constant that describes the exponential loss of phase coherence due to very small variations in the magnetic field caused by interactions between the spinning nuclei. In principal, $T_{2}$ can be measured from the decaying voltage induced in the detector:

$$
M=M_{0} \exp \left(-t / T_{2}\right)
$$

In practice, the spin-spin relaxation is not the only factor that causes the decay of transverse magnetization. There are always some local variations in the external magnetic field, these static field inhomogenities give rise to a spread of precession frequencies within the sample (Leach 1988) which is an important additional 
contribution to different phases. The total decay time which is a measure of all the factors leading to dephasing is called $\mathrm{T}_{2}{ }^{*}$ It is found that

$$
\frac{1}{T_{2} *}=\frac{1}{T_{2}}+\gamma \frac{\Delta B_{0}}{2}
$$

Where $\Delta \mathrm{B}_{0}$ is the variation in the static magnetic field over the sample.

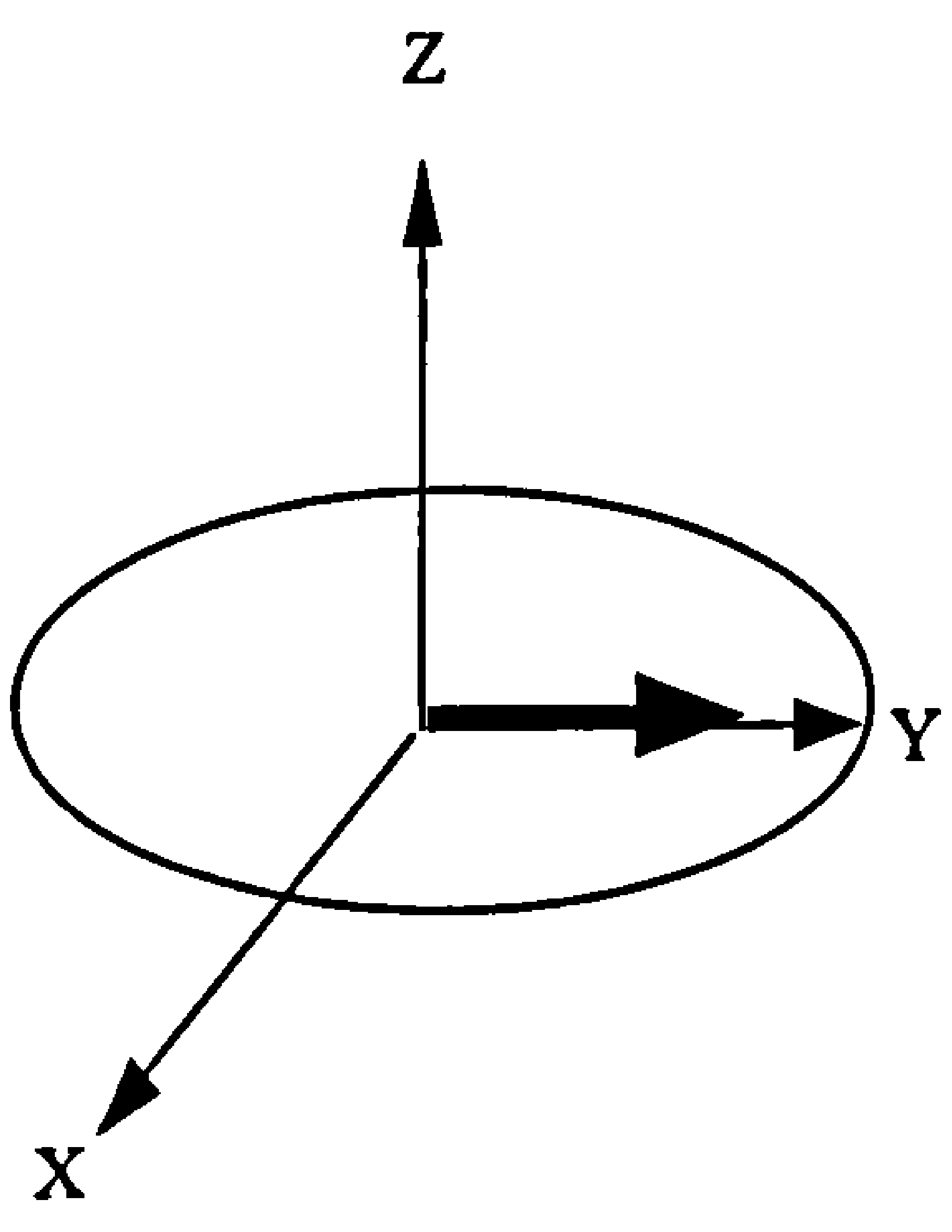

(a)

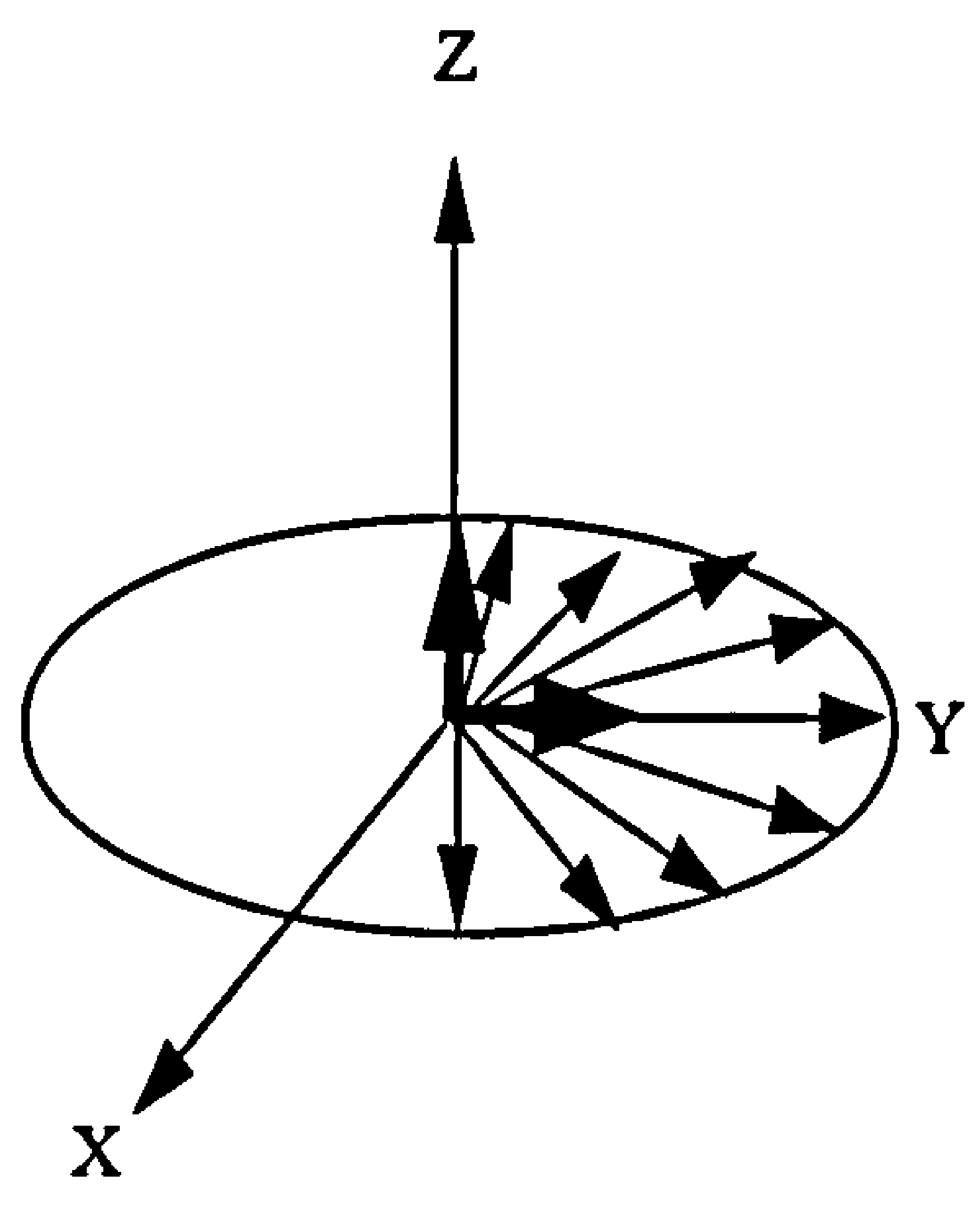

(b)

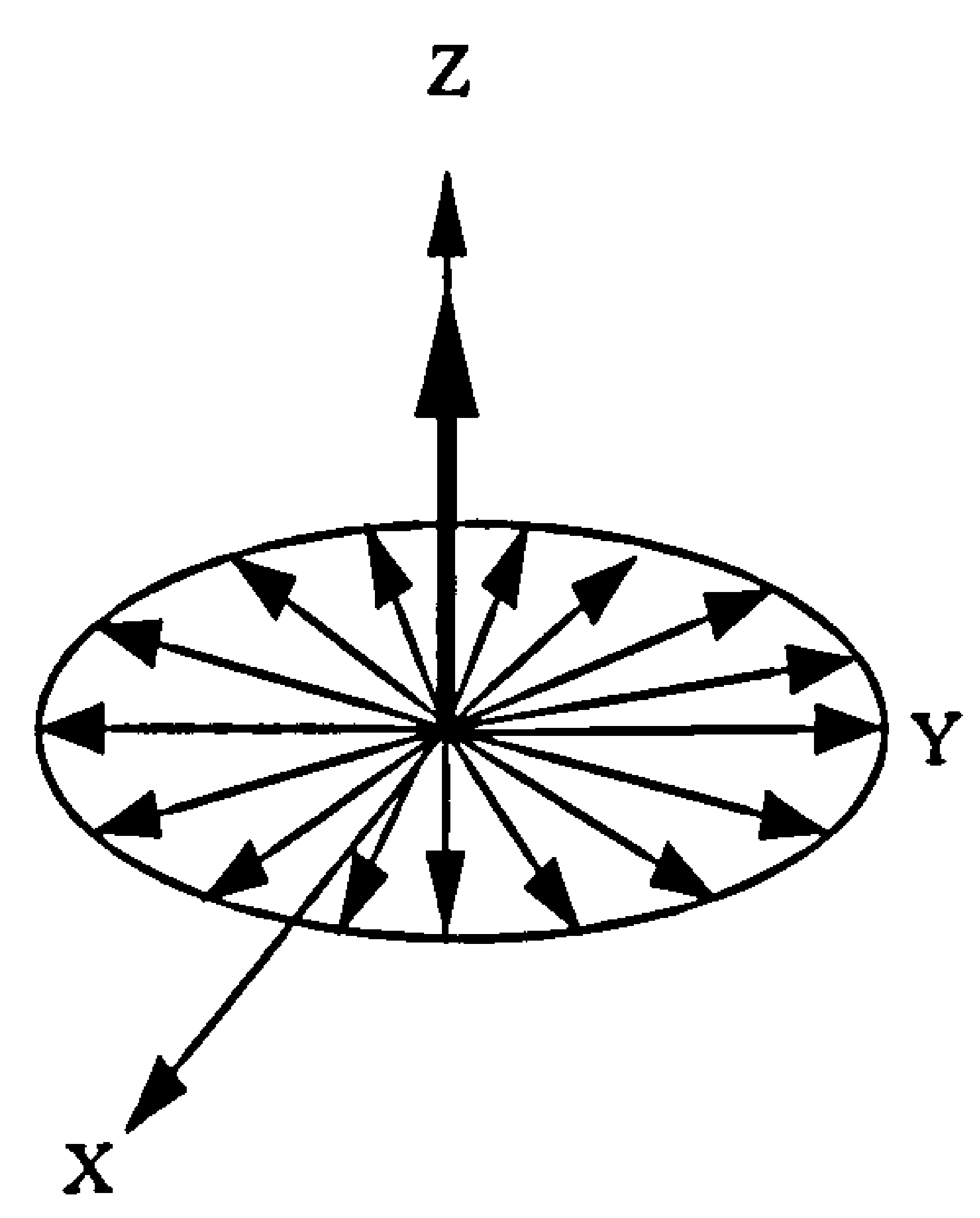

(c)

Figure 2.5. Transverse $\left(\mathrm{T}_{2}\right)$ relaxation. (a) After $90^{\circ}$ pulse, $\mathrm{t}=0$;

(b) at time $\cong T_{2} ;(c) t \rightarrow \infty$

\subsection{Pulse sequences}

The signal for magnetic resonance imaging is obtained following exposure of all the spins to a series of RF pulses and application of magnetic field gradients. The characteristics of these pulse sequences can be used to evaluate many different magnetic properties of the tissues. For many years the most commonly used sequences in magnetic resonance were the spin-echo and inversion recovery sequences. A pulse sequence consists of several components, the main ones are as follows: 
The Repetition Time (TR) is the time from the application of one RF pulse to the application of the next RF pulse and is usually measured in milliseconds. The TR determines the amount of longitudinal relaxation that is allowed to occur between the end of one RF pulse and application of the next.

The Echo Time (TE) is the time from the application of the RF pulse to the peak of the signal induced in the coil in a spin-echo sequence (see later). It is also usually measured in milliseconds. The TE determines how much decay of transverse magnetization is allowed to occur before the signal is read.

The time of Inversion (TI) is the time in between the $180^{\circ}$ and $90^{\circ} \mathrm{RF}$ pulse in the inversion recovery sequence (see later). After the $180^{\circ}$ pulse there is a delay during which the nuclei will return towards equilibrium. The application of a $90^{\circ}$ pulse during this return to equilibrium produces an NMR signal whose amplitude contains $\mathrm{T}_{1}$ information.

The signal strength or brightness of a MR image depends on the timing parameters of the pulse sequence, the relaxation time constants of the sample $\left(T_{1}\right.$ and $T_{2}$ ), the proton density ( $\rho$ ), the motion of the sample (e.g. blood flow), and the properties of the imaging system such as the coil design. Therefore signal intensity for each type of pulse sequence can be written as

$$
S=f\left(T_{1}, T_{2}, \rho, T E, T I, T R\right)
$$

Alterations of these parameters results in changes in signal intensity and consequently apparent contrast changes in MR images (Hardy et al 1985, Kjos et al 1985). There also are several sources of non uniformity in the signal intensity i.e. non uniform RF pulses, magnetic field instabilities and inhomogenities, and uncompensated gradient eddy currents (Kjær et al 1987, Simmons et al 1994).

The following section describes some of the pulse sequences that are important in NMR imaging. 


\subsubsection{Saturation recovery}

Pulse sequences that do not allow magnetization to fully recover are called saturation or partial saturation recovery pulse sequence. This is achieved by using a short pulse repetition time (TR). Excitation pulses are repeatedly applied to the sample and the NMR signal which is the free induction decay (FID) is measured after each RF pulse (Figure 2.6).

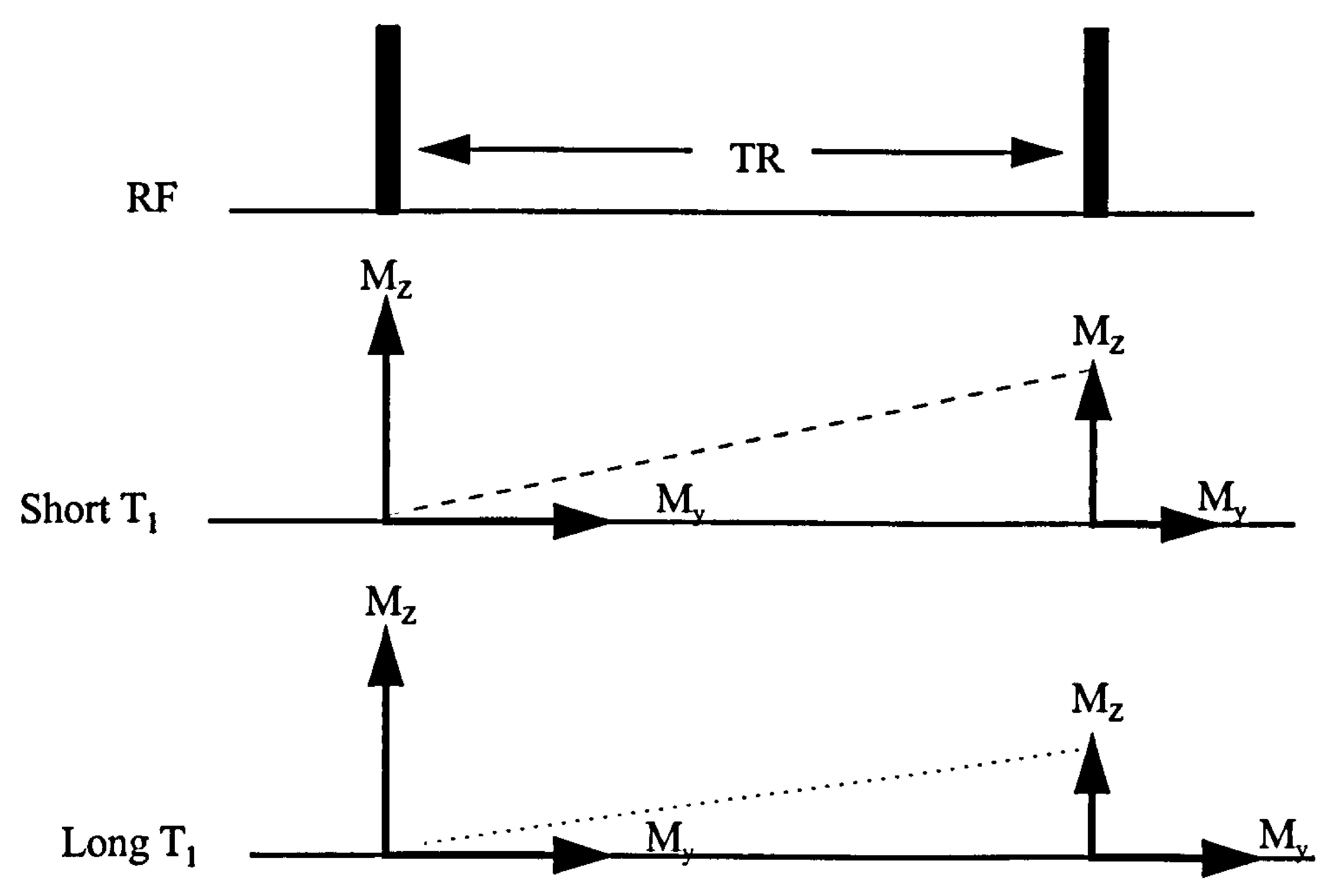

Figure 2.6. The short $T_{1}$ sample produces a larger NMR signal than the long $T_{1}$ sample for the same $T R$

If a long $T R$ is used (relative to $T_{1}$ ) the nuclei have enough time to return to equilibrium before the next RF pulse. Therefore the amplitude of the NMR signal depends on the water content of the volume being examined and only contains proton density information. If, however, the TR is selected to be much shorter, the nuclei are not given sufficient time to return to equilibrium before the next RF pulse. Therefore their $\mathrm{Z}$ component of magnetization is reduced and consequently the NMR signal is smaller. Thus the amplitude of the NMR signal will contain information about the $T_{1}$ of the sample as well as the proton density. 


\subsubsection{Spin-Echo sequence}

We have already seen that the NMR signals decay because the spins lose their phase coherence. This results from the minor random variations produced by the spins themselves as well as variations in the external magnetic field applied to the sample. The spin-echo pulse sequence is primarily used to cancel out magnetic field inhomogeneity. Therefore this sequence makes it possible to investigate the true intrinsic $T_{2}$ relaxation time of a tissue and avoid instrument related effects. Following the application of a $90^{\circ}$ pulse the net magnetization is rotated to the transverse plane and spins precess in phase. When the $90^{\circ}$ pulse is switched off a FID is generated but the signal is not recorded. With time some spins will be precessing faster and others slower due to inhomogeneities in the magnetic field and in the intrinsic $T_{2}$ relaxation parameter. This results in a decreasing transverse magnetization and thus loss of signal. If, after a short time, a $180^{\circ}$ pulse is applied the phase of the spins will be reversed so that those that are precessing faster will fall behind the slower precessing spins. Therefore the effect of the inhomogeneities in the magnetic field on the loss of phase coherence of the spins is reversed. The faster spins continue precessing and catch up with the slower spins and they gradually precess back into the phase for a short time producing a stronger transverse magnetization and thus an NMR signal. The time delay between the $90^{\circ} \mathrm{RF}$ pulse and the signal is called the echo time (TE). The signal of the echo is a function of the intrinsic $T_{2}$ relaxation time and magnetic characteristic of the tissue, and not a reflection of the quality of the external magnetic field itself.

The typical spin-echo pulse sequence (Figure 2.7) used for clinical imaging contains usually one or more $180^{\circ}$ pulses. In NMR tomograms where the spin-echo sequence is used both $T_{2}$ values and the proton density of the tissue contribute to the final image. 


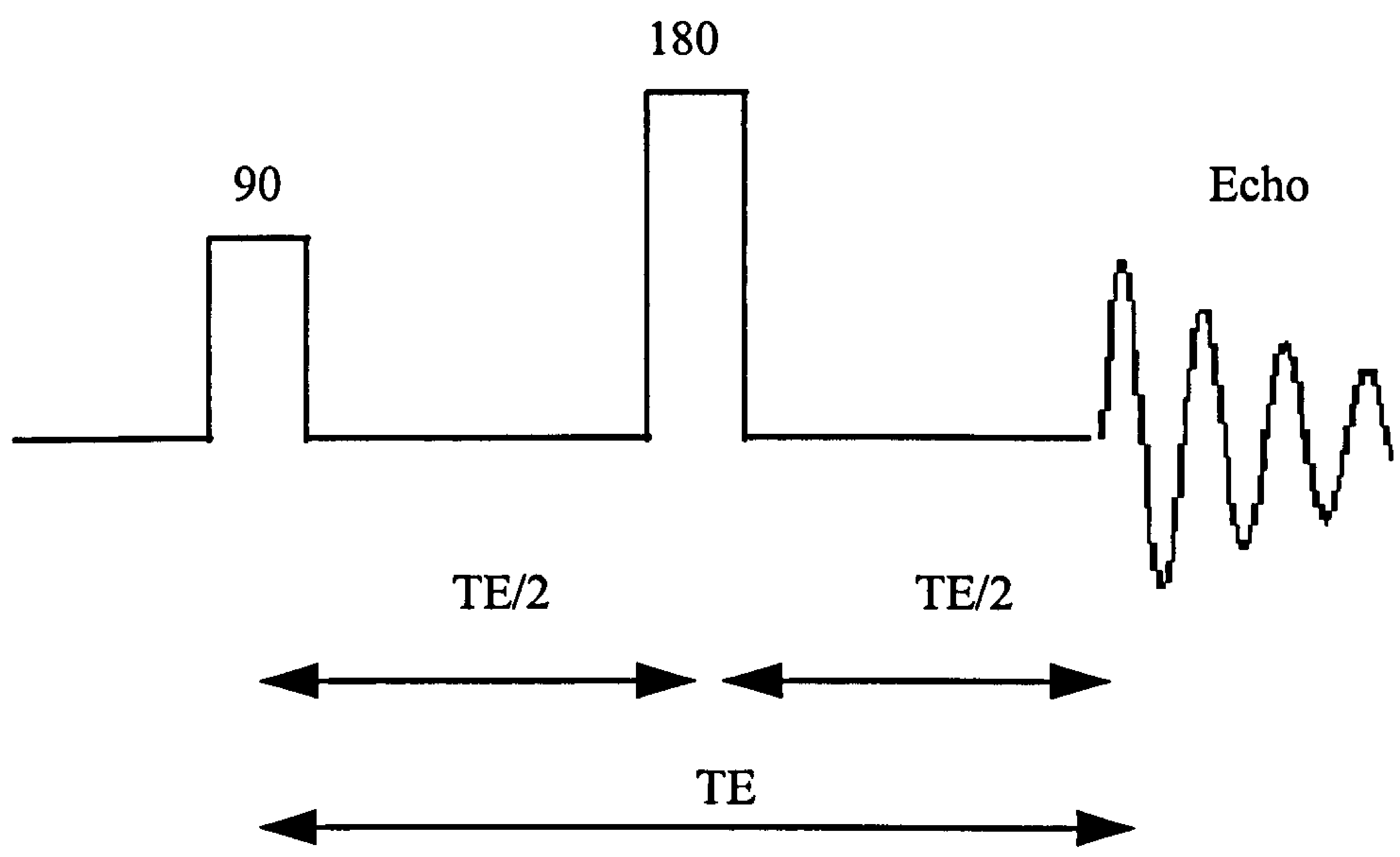

Figure 2.7. Spin echo-pulse sequence

\subsubsection{Inversion recovery}

The inversion recovery (IR) sequence is initiated by a $180^{\circ}$ pulse and as is apparent from the name this completely inverts the longitudinal magnetization. This pulse is similar to the $180^{\circ}$ pulse used in the spin-echo sequence but with a different effect on the net magnetization, because the magnetization initially is in a different state. Assuming that the spins are at equilibrium and the net magnetization is aligned with the external field, then with a $180^{\circ}$ pulse, all of the spins that were parallel become anti-parallel. The net magnetization is therefore large and negative rather than zero. Over time as the number of anti parallel spins decreases the number of parallel spins increases and the spins then return to equilibrium. No signal is generated since there is no transverse magnetization. In order to obtain an MR signal, the IR sequence uses the same pulses as a spin-echo pulse sequence after the initial pulse. Following a $180^{\circ}$ pulse and before the spins return to complete equilibrium a spin-echo technique, $90^{\circ}$ and $180^{\circ}$ pulses are applied to the sample (Figure 2.8). The transverse magnetization that is produced is proportional to the longitudinal magnetization at the time of the $90^{\circ} \mathrm{RF}$ pulse. Therefore the time delay between the 
$180^{\circ}$ pulses determines the amount of $\mathrm{T}_{1}$ information contained in the amplitude of the NMR signal. The time between the $180^{\circ}$ and $90^{\circ} \mathrm{RF}$ pulse is called the inversion time (TI).

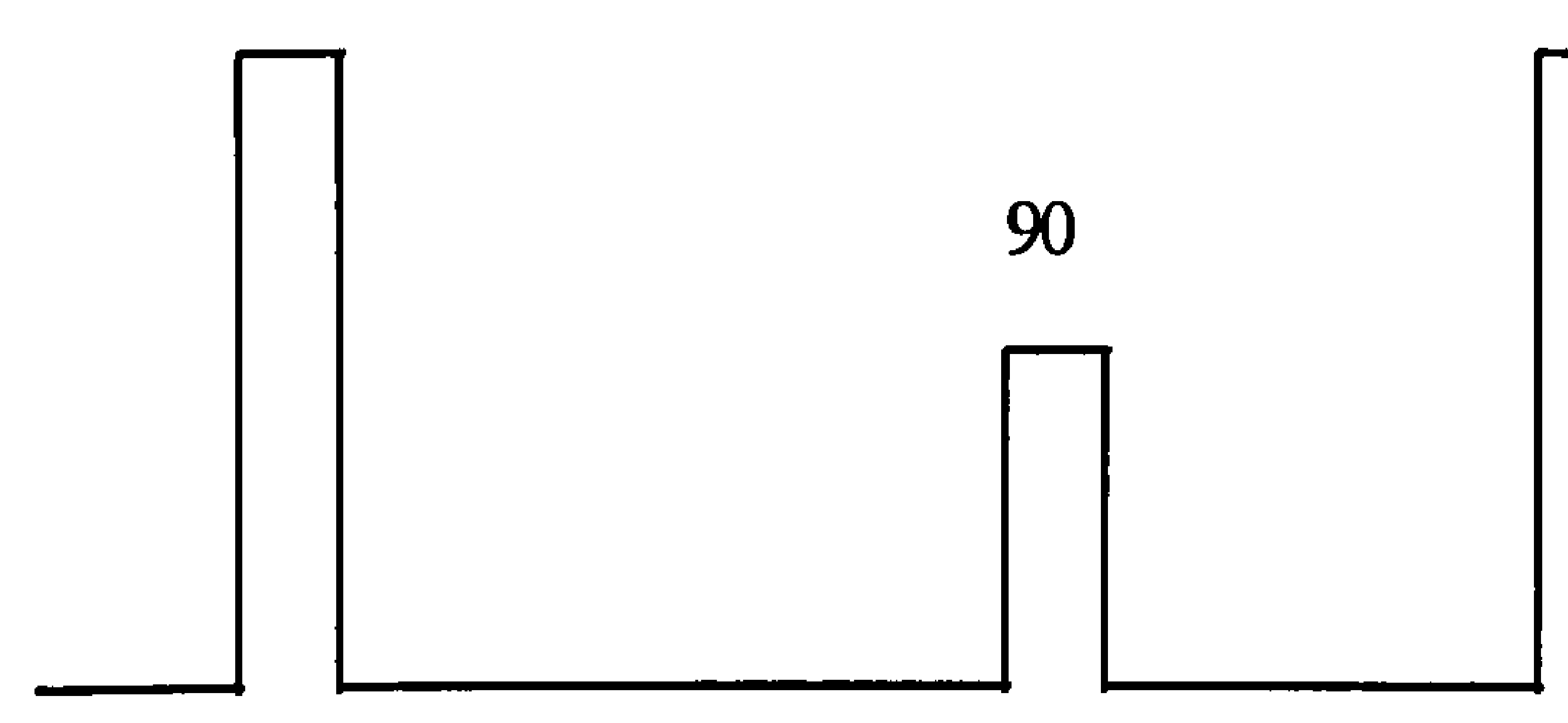

$\mathrm{TE} / 2$ $\mathrm{TI}$
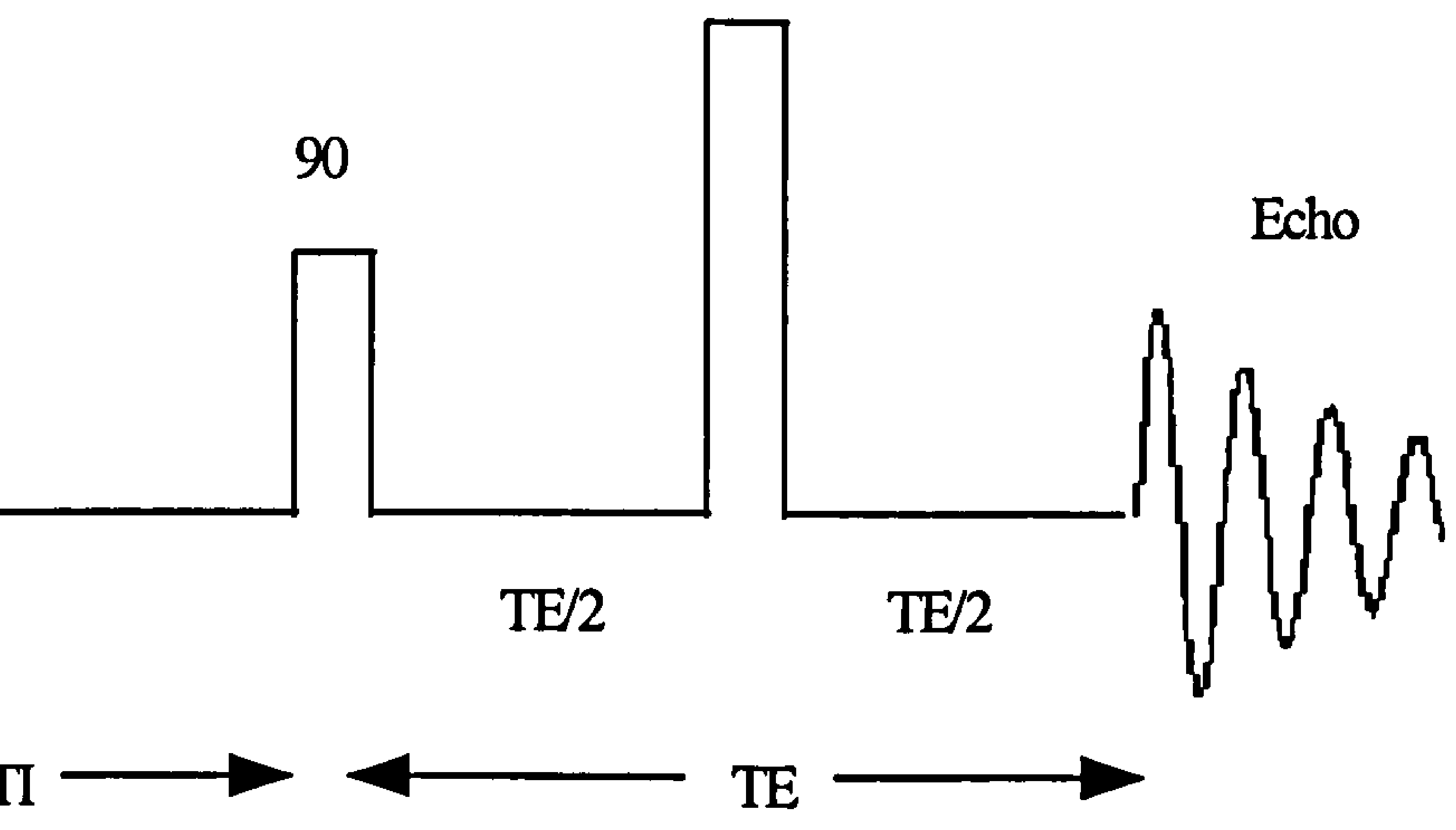

Figure 2.8. Inversion recovery pulse sequence

\subsection{Relaxation measurements}

\subsubsection{Measurement of $T_{1}$ relaxation time}

Longitudinal relaxation can not be detected directly. It can be measured by the use of saturation recovery or inversion recovery sequences. Among these sequences the most commonly used technique for accurate $T_{1}$ measurement is the inversion recovery method. The full sequence is

$$
\left[180^{\circ}-\mathrm{TI}-90^{\circ}(\mathrm{FID})-\mathrm{TR}\right]_{\mathrm{n}}
$$

where a very long TR is used, typically seven times larger than the longitudinal relaxation time, the system completely returns to equilibrium at the beginning of each TR. By manipulating the inversion time interval the longitudinal magnetization is sampled at different times. The longitudinal magnetization can range from completely 
inverted to equilibrium (completely parallel spin state). In order to calculate $T_{1}$ the amplitude of the signal is derived from

$$
S(T I)=S_{0}\left(1-2 \exp \left(-T I / T_{1}\right)\right.
$$

for different values of TI. By plotting the value of S (TI) against TI, $T_{1}$ can be determined.

\subsubsection{Measurement of $T_{2}$ relaxation time}

As has been noted earlier, the two factors that contribute to the FID following a $90^{\circ}$ pulse are external magnetic field inhomogenities and the intrinsic magnetic field variation inside the sample. Therefore the envelope of the FID decays with $T_{2}{ }^{*}$ rather than $T_{2}$ relaxation time. In order to overcome this problem and measure the intrinsic transverse $T_{2}$ relaxation time of the tissue, it is necessary to remove the dephasing caused by magnetic field inhomogeneity, leaving the effect of internal random processes. The method that is usually used for this purpose is the spin-echo sequence and was introduced by Hahn in 1950. He explained that the phase of a spin can be reversed by the application of a $180^{\circ}$ pulse and the effect of external field inhomogenities, to a large extent, is removed. In the Hahn spin-echo, however, the intrinsic rate of dephasing resulting from the sample properties can be measured providing that there is no diffusion of protons. The simple pulse sequence in spinecho is

$$
90^{\circ}-\tau-180^{\circ}-\tau-\text { echo }
$$

By changing $\tau$, that is the time delay between $90^{\circ}$ and $180^{\circ}$, the spin echo signal can be measured at different times and therefore it is possible to measure the magnitude of the transverse magnetization at any desired echo time. In practice, diffusion of the spins does occur and causes the nuclei to experience different magnetic fields through 
the inhomogeneous external field. Therefore, the precision of the $T_{2}$ measurement is affected by diffusion.

To overcome this problem Carr and Purcell (1954) introduced a modified method of Hahn spin-echo (CP sequence). In this method they applied successive $180^{\circ}$ pulses after a $90^{\circ}$ pulse and recorded a signal for each rephasing pulse (figure 2.9).

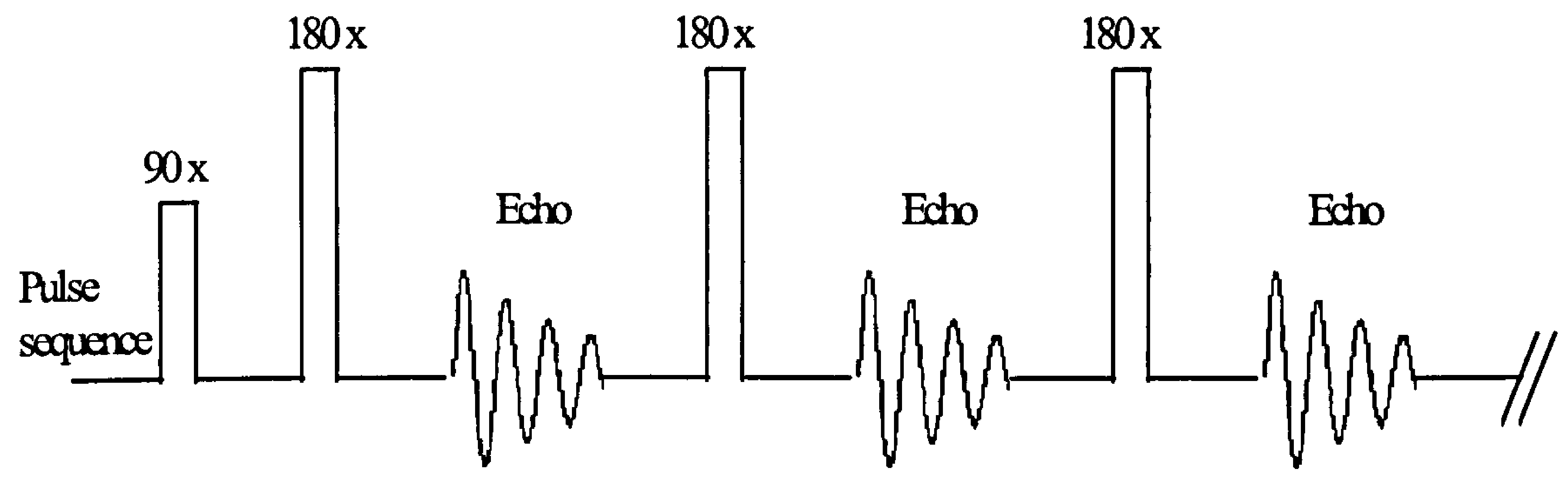

Figure 2.9. The CP pulse sequence

By plotting the amplitude of the echoes obtained, transverse relaxation time can be measured within a single repetition of the sequence. This sequence can be described as

$$
90^{\circ}-\left(\tau-180^{\circ}-\tau-\text { echo }\right)_{n}
$$

For the application of the $\mathrm{CP}$ sequence it is necessary to have an accurate amplitude for the $180^{\circ}$ pulse. If there is a small deviation from the exact $180^{\circ}$ rotation angle it will cause an accumulating error in the result (Meiboom and Gill 1958). Also, the number of $180^{\circ}$ pulses should be large to eliminate the effect of diffusion (Carr and Purcell 1954).

The CP sequence was modified by Meiboom and Gill (1958) this modification is known as the CPMG sequence. In this method the phase of the initial $90^{\circ}$ pulse is shifted by $90^{\circ}$ relative to the phase of the $180^{\circ}$ pulses (figure 2.10). In other words the $90^{\circ}$ pulse is oriented along the $\mathrm{y}$ axis while $180^{\circ}$ pulses are directed along $\mathrm{x}$ axis. This modification results in the formation of the echoes avoiding cumulative errors. 


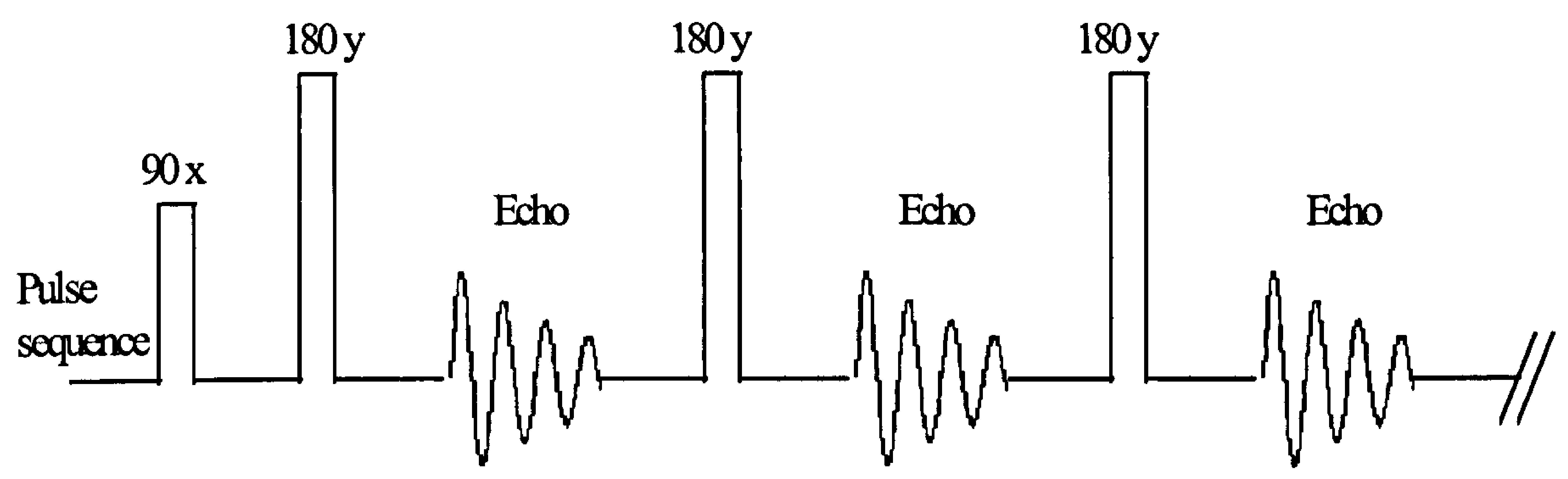

Figure 2.10. The CPMG pulse sequence

In order to measure the $T_{2}$ relaxation time accurately, a series of spin-echo images is essential. This series of images is acquired using increasingly longer TE time while keeping the TR and all other imaging factors constant. By measuring the intensity of the signal and plotting it against the TE time, the $T_{2}$ relaxation time can be calculated.

\subsection{NMR Imaging}

So far, an NMR signal arising from the whole of an object inside the magnet has been discussed. However, what is really required is an image that shows the spatial distribution of nuclear spins (in specific planes).

NMR imaging is an extremely flexible technique and offers numerous ways in which the object may be imaged (Lerski 1985). This is possible because a welldesigned imaging system will allow the implementation of any of the possible imaging methods without changes in hardware as reported by Taylor and Inamdar (1985).

All NMR imaging techniques exploit the property that the Larmor frequency is proportional to the applied magnetic field strength. A spatial variation of magnetic field will thus enable the position of the spins to be identified from the precessional frequency. Therefore positional information which is necessary to form an image of the object can be obtained by applying magnetic field gradients. 


\subsubsection{Gradient fields}

As already mentioned spatial information is encoded in the MR signal by altering the magnetic field strength across the imaging volume. This can be done by superimposing on the main field a magnetic field gradient the so-called field gradients $\left(G_{x}, G_{y}, G_{z}\right)$ by which the field varies in a linear manner along a particular axis. The magnetic field is therefore stronger or weaker in some places than in others. This results in different precessional frequency experienced by spin at different positions and, according to the Larmor relation, they will have different resonant frequencies (Figure 2.11).

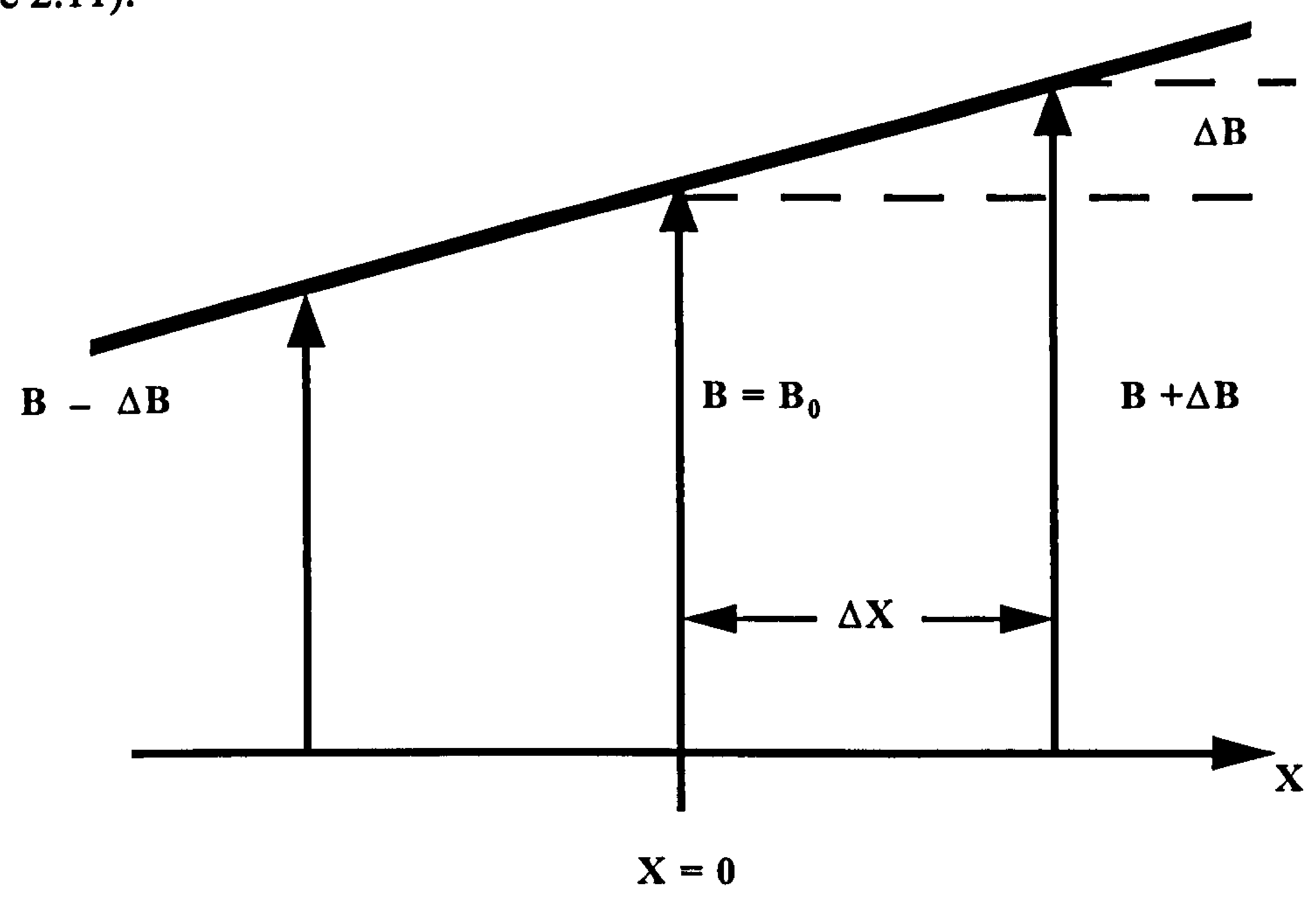

Figure 2.11. Magnetic field gradient

The resonance frequency is equal to the base resonance frequency of the magnetic field plus the change in frequency caused by the gradient.

$$
B(x)=B_{0}+\frac{\mathrm{dB}}{d x} \cdot x
$$

or

$$
B(x)=B_{0}+G_{x} \cdot x
$$


where $G_{x}$ is the magnetic field strength in the $x$ direction. So the resonance frequency can be written

$$
\omega(\mathrm{x})=\gamma \mathrm{B}_{0}+\gamma \mathrm{G}_{\mathrm{x}} \cdot \mathrm{x}
$$

At the centre, the magnetic field is not affected by the gradient since the field change caused by the gradient is zero at the centre.

The gradient fields are generated by the linear gradient coils located within the magnet. The gradient coils are switched on for a short time to produce transient magnetic field that differs from the main magnetic field. Although the strength of the magnetic field produced by the gradient coil is far less than that of the main magnetic field, it is strong enough to generate a measurable change in the frequency or phase of the signal.

There are three gradient coils within the bore of the magnet in order to obtain complete spatial localization of the object within the magnetic field. The three gradient directions are aligned with the magnet axes as shown below. The gradient directions are mutually orthogonal (Figure 2.12).

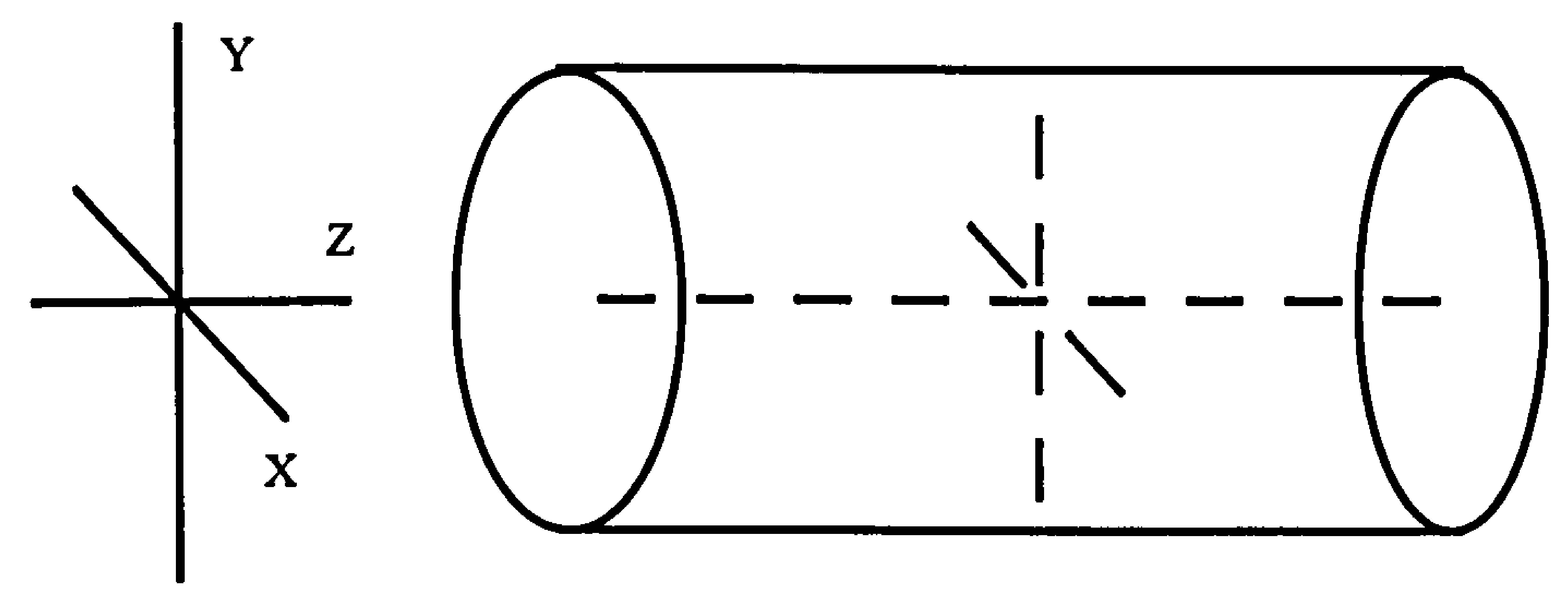

Figure 2.12. The $\mathrm{X}, \mathrm{Y}$ and $\mathrm{Z}$ gradient axes

Gradients perform three tasks encoding the image: 
1) Slice selection, selects position of the slice (the scan plane).

2) The frequency-encoding gradient encodes positional information in one direction in the image slice.

3) The phase-encoding gradient encodes positional information in a direction perpendicular to the frequency-encoding direction.

The application of all the gradients selects an individual slice and produces a frequency shift along one axis and a phase shift along the other. The system can now record the individual signals which are encoded with their location within the magnet and convert the signal into an image.

\subsubsection{Image Acquisition and reconstruction}

\subsubsection{Projection reconstruction}

Projection reconstruction is the technique that was used to produce images in early NMR experiments. In this technique the production of NMR images is based on reconstruction of several projections that are taken from the object.

As with most imaging methods, projection reconstruction requires the preselection of an imaging plane within the object. This can be achieved by application of magnetic field gradient and suitable RF pulse. When a linear magnetic gradient is applied to the object along the $\mathrm{Z}$ axis for example, the precessional frequency of the spins in the object will differ relatively along $Z$. If therefore, a selective RF pulse with a narrow range of frequency is applied, it will only excite the spins in the desired cross section (Figure 2.13). Thus the spins in the selected section resonate and give rise to an NMR signal that will contribute to the image. The thickness of the slices is dependent either on the range of $R F$ frequencies or the strength of the gradient. 


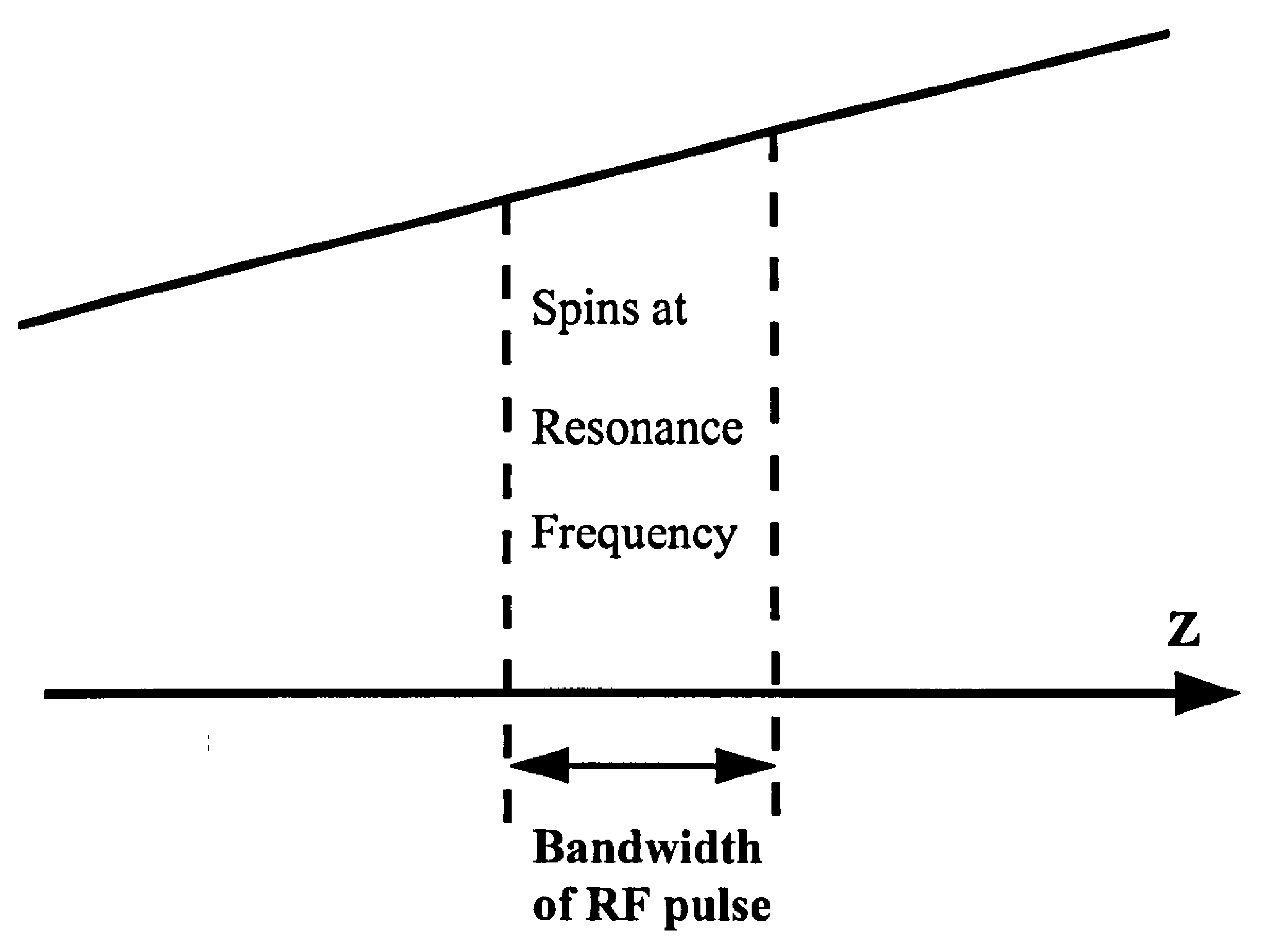

Figure 2.13. Selection of the slice in the presence of a field gradient in the $\mathrm{Z}$ direction

Once a slice has been selected, the next step is to encode spatial information within the plane. Spatial information is encoded in one direction using another magnetic gradient known as frequency-encoding gradient. This gradient is applied perpendicular to the $\mathrm{z}$ axis while the NMR signal is being received and may also be called the readout gradient. This alters the magnetic field strength and consequently the precessional frequency of signal in a linear fashion along the direction of the gradient. The signal now is encoded according to its frequency. Thus the Fourier transform of the FID will be the projection of the signal intensity in the plane onto the frequency-encoding axis.

The combination of $\mathrm{x}$ and $\mathrm{y}$ gradients during read out of the signal can produce projections of the signal intensity at different angles (Taylor et al 1988). Therefore by repeating the sequence of slice selection and using a series of $x y$ gradient combinations that change for each measurement (figure 2.14), a set of projections can be produced. An image then can be reconstructed from these projections using 
conventional back projection techniques similar to those used in X-ray computed tomography (CT).
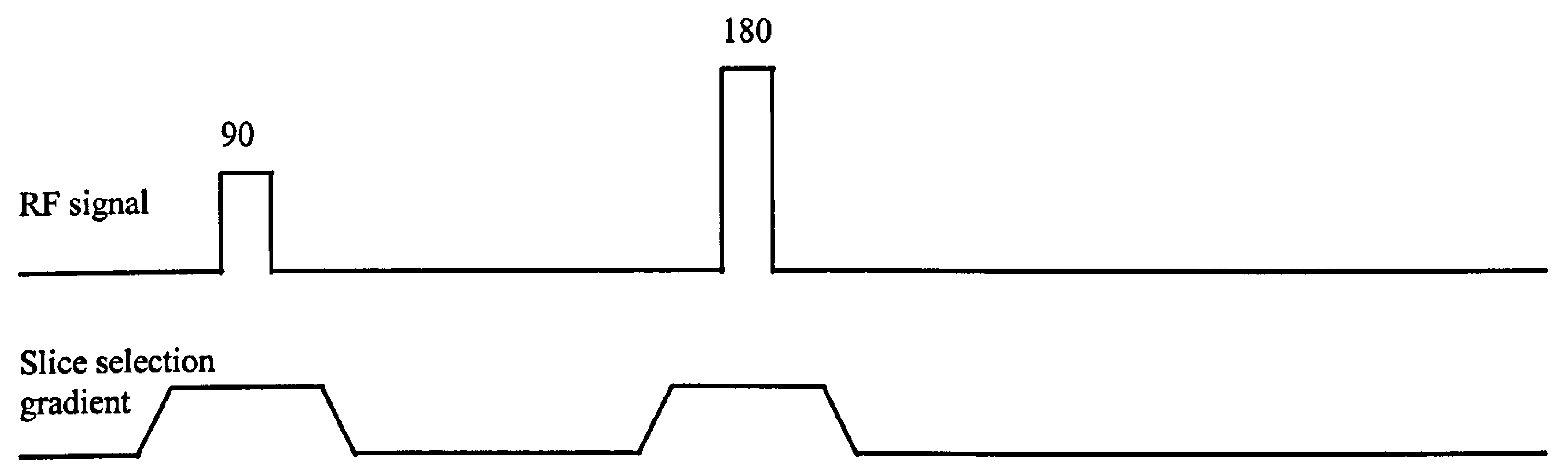

Frequency encoding gradient $x$ axis
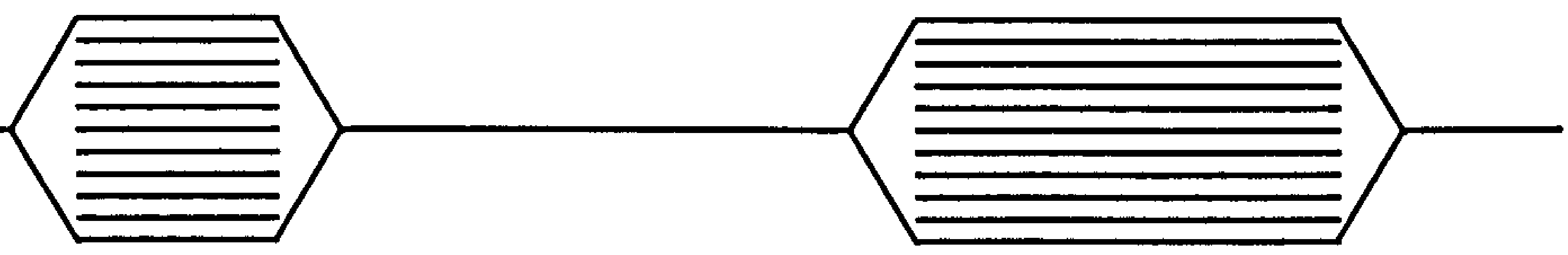

Frequency encoding gradient $\mathrm{y}$ axis
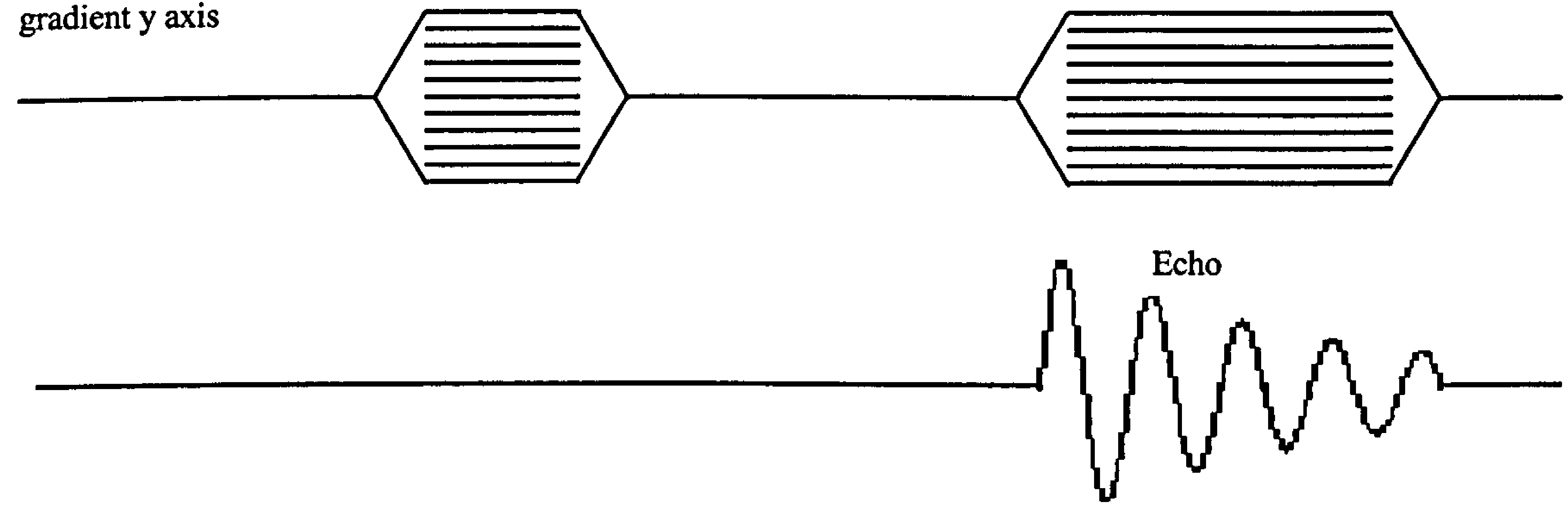

Figure 2.14. A spin-echo sequence in the projection reconstruction technique

\subsubsection{Two-dimensional Fourier Transformation}

Two-dimensional Fourier transform (2DFT) is the technique that is now commonly used to produce NMR images. Selection of a slice in this method is the same as the projection reconstruction method. Instead of combining the xy gradient as in the projection method, this method uses the frequency encoding gradient $(x)$ to store spatial information in one dimension and the phase encoding gradient $(y)$ in the 
other dimension. In order to see how spatial information is encoded in the y axis, the effect of the phase gradient will briefly be discussed.

When the phase encoding gradient is on, the precessional frequency of the spin is changed along the $y$ axis. The spins in the part of the higher magnetic field strength will precess faster than those in the lower part. This results in varying phase angle throughout the $y$ axis and the phase angle being larger at the region of higher magnetic field strength than the lower region. The phase differences or shift between the spins is proportional either to the length of time that the gradient is on or the amplitude of the gradients. When the $\mathrm{Y}$ gradient is off all spins along the $\mathrm{y}$ axis precess again at the same frequency but the phase difference remains fixed.

In order to create an image with the 2DFT technique, following the slice selection gradient, the phase encoding gradient is switched on for a short period and the signal is recorded in the presence of the read-out gradient. The sequence is repeated a number of times, increasing the amplitude of the y gradient while holding the $\mathrm{x}$ axis gradient constant (figure 2.15). A set of signals is produced, one for each amplitude of the phase-encoding gradient. The complete imaging sequence then consists of collecting several data points for each number of changes in amplitude of $y$ gradient. If these data points are placed in the rows of a matrix their Fourier transformation in two dimensions will produce the spatial distribution in the $\mathrm{x}$ and $\mathrm{y}$ direction and consequently the final image. 


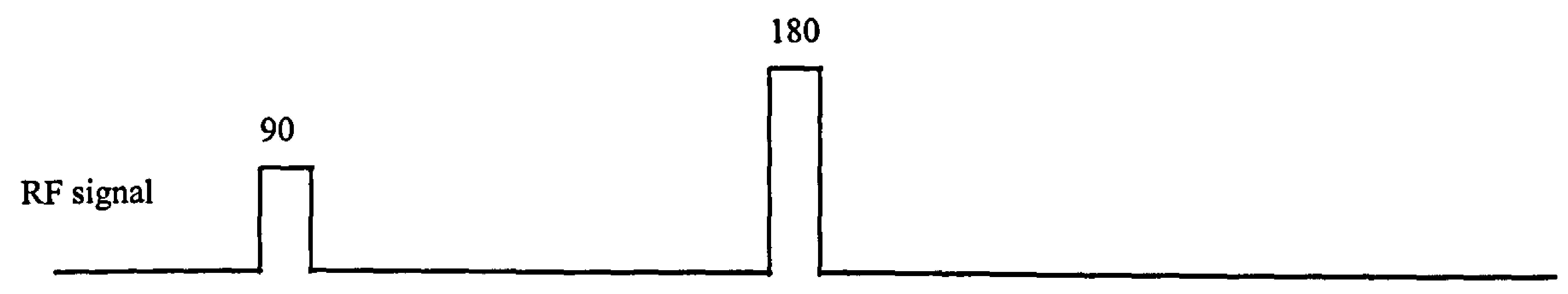

\section{Slice selection}

gradient

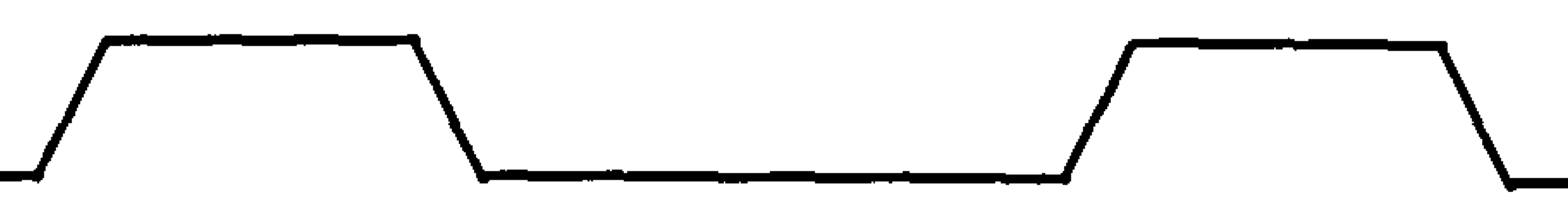

Frequency encoding gradient
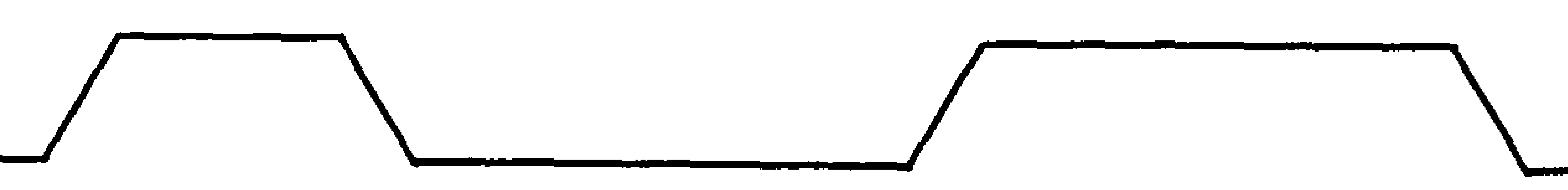

Phase encoding gradient
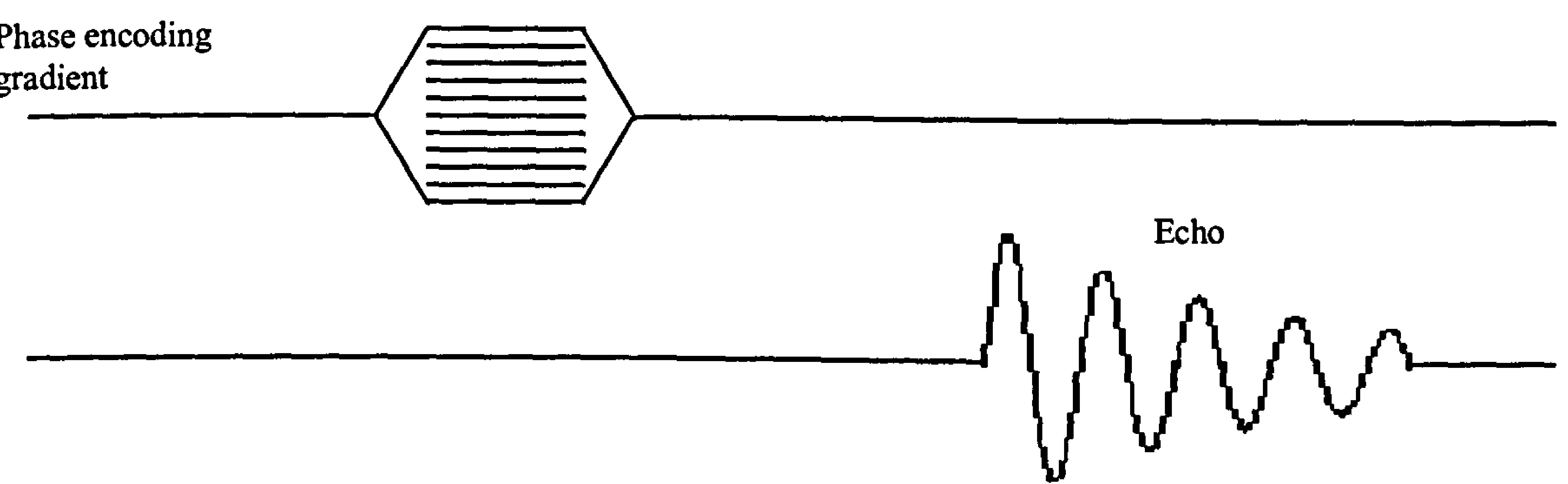

Figure 2.15. A spin echo sequence in the 2DFT imaging technique 


\section{Chapter 3 . BANG Gel Dosimetry}

\subsection{Introduction}

Up until 1993 ferrous sulphate infused gel (Gore et al 1984a) was the only dosimetric system that involved MRI in the measurement of radiation dose distributions in radiotherapy. Although this system provided a number of unique features for dosimetry purposes, the major drawback was the diffusion of the ferric ions following irradiation. This led to the loss of spatial resolution and blurring of the image, which in turn limited the number of images, the time between irradiation and imaging and the doserate that could be used. A gel system that did not suffer from this problem was needed.

In 1993 and 1994 alternative types of gel with the acronyms BANANA and BANG were introduced by Maryanski and co-workers. These were based on polyacrylamide that is formed by radiation-induced co-polymerization and crosslinking of acrylamide and bis-acrylamide monomers in a gel matrix. The reaction is vinyl addition polymerization initiated by free radicals (Bio-rad Bulletin 1987). The formation of the polymer in the gel following irradiation increases the relaxation rate of the neighbouring water protons in proportion to absorbed dose. This increase in relaxation rate can be detected by MRI.

The main difference between the BANG gel, the subject of this study and the BANANA gel is the gel matrix used. The former uses gelatin, the latter agarose. The reasons for using gelatin rather than agarose are:- 
1. NMR relaxation rates of water protons in gelatin are an order of magnitude less than agarose (Ablett et al 1978, Olsson et al 1989). This results in a lower background relaxation rate $\left(R_{2}\right)$ in the gel which increases the dynamic range of the measurement (Maryanski et al 1994).

2. The preparation of the gelatin is more simple than agarose especially for volumes beyond $1000 \mathrm{ml}$ (Olsson et al 1989, Prasad et al 1991).

3. The cooling rate of the gelatin is much smaller than that of agarose giving a more homogeneous gel (Duzenli 1994, Maryanski et al 1994)

4. Gelatin is more transparent than agarose allowing the polymerized area to be visualised more clearly (Maryanski et al 1994).

\subsection{Nature of the polymer}

The word polymer is derived from the Greek words "poly" meaning many and "meros" meaning part. This term is applied to a long molecule that contains a chain of several thousand atoms held together by covalent bonds. This large molecule is produced through a process of polymerization where monomer molecules chemically react together to build polymer chains. These chains may be linear, slightly branched or interconnected to form three-dimensional networks. If the polymer is formed by employing only one type of monomer the resulting molecule is called a homopolymer. In the case when different types of monomer species are used the polymer is named a co-polymer (Young 1981). Polymers are said to be linear if the repetition of the repeating units in the molecules is similar to that of the links in chain. Polymer molecules of this type are often referred to as chain molecules or as polymer chains. 
The size of such polymer chains may be defined in terms of the degree of polymerization, the number of repeating units in the chain. An alternative parameter to describe the size of the polymer molecule is the molar mass of one mole of the polymer. Covalent chemical bonds that occur between macromolecules are known as cross links. Separate linear or branched chains may be joined together along the chains by cross links to give a cross-linked polymer. More extensive cross linking may lead to the formation of three-dimensional cross linked or network polymers.

Polymers are characterized, and differ from one another, by the chemical and physical nature of the repeating units in the chains (Morton-Jones, 1989). Their presence and density have a profound influence on both the chemical and mechanical properties of the material in which they occur (Nicholson 1991).

\subsection{Properties of the polymer gel}

A polyacrylamide gel is a cross-linked polymer network suspended in a liquid medium. NMR relaxation characteristics of cross-linked gels are often similar to tissue. They can therefore be used to provide a suitable system to model relaxation effects of tissue and quantitate the degree to which the macromolecular matrix influences water relaxation (Kennan et al 1992). It has been observed that in polyacrylamide or other macromolecular gels such as agar or gelatin, water consists of a large amount of "bulk" water and a small amount of "associated" water (Katayama and Fujiwara 1979). In "bulk" water the motion of the protons is not restricted and it is therefore a free state, whereas the protons in the "associated" water are restricted. This occurs because gels are constructed from a macromolecular network containing a 
number of small cavities, therefore the motion of the water molecules in the cavities are confined compared to the molecules in pure water. This results in enhanced relaxation in the molecules of bound water due to restricted mobility of the “associated" water molecules. In practice, however exchange between these two states of water is normally rapid and one cannot separate the NMR signals. Therefore the relaxation time is a measure of the average molecular properties. As the relaxation times of water in the gel are shorter than that of the "bulk" water this can therefore give an indication of the presence of the "associated" water (Katayama and Fujiwara 1979).

\subsection{Radiation-induced polymerization}

When ionising radiation passes through matter it transfers energy to the molecules of the absorbing material by various physical mechanisms. This transferred energy results in the ionisation and excitation of the atoms and the formation of secondary charged particles which in turn interact with the electrons of the surrounding molecules. The observed chemical changes result from this deposition of energy by the incident radiation.

Radiation-induced polymerization is a chain reaction in which a large number of chemical changes may follow each single act of ionisation or excitation. Polymerization of a monomer consists typically of three different steps, initiation, propagation and termination. These steps may be modified by other reactions such as transfer, in which polymerization process continue by generation of a new radical. The role of radiation in the polymerization process is essentially confined to the initiation step, where it is considered as a substitute to the chemical initiator of the reaction 
(Charlesby 1987). Polymerization can be initiated by free radicals or ions. Since radiation can produce both free radicals and ions it can initiate polymerization by both means. It should be noted, however that the reactions are mostly initiated by free radical mechanisms (Williams 1968). In this method free radicals that are produced by radiation react with monomers containing a double bond to form an active centre which is capable of reacting with further monomer molecules to give a macromolecular chain.

\subsubsection{Kinetics of radiation polymerization}

The kinetics of polymerization are built on the idea that three distinct stages are involved in such reactions. The initiation takes place in two steps each with its own rate constant. First radiation produces free radicals $(\mathrm{R} \cdot)$ which subsequently interact with monomer molecules (M) to form active centres. An active centre is the site from which further polymerization is produced. The rate of active centre formation is controlled by the rate of radical formation.

$$
\mathrm{R} \cdot+\mathrm{M} \rightarrow \mathrm{RM} \cdot
$$

Following initiation, propagation takes place in which many more monomers are added successively to the growing chain. It is assumed that the rate of propagation is independent of the size of the growing polymer molecule. The average life-times of the growing chain are extremely short and several thousand additions can take place within a few seconds (Young 1981). 


$$
M \cdot n+M \rightarrow M \cdot(n+1)
$$

Finally in the termination step two growing chains may react with each other to form one or more polymer molecules. This is known as the combination mechanism. Alternatively a hydrogen atom can be transferred from one chain to other to form two polymer chains. This mechanism is called disproportionation. These two mechanisms of termination often take place simultaneously. Therefore, except where it is necessary to distinguish between the two mechanisms, the rate constants are generally combined into a single rate constant.

$$
\begin{aligned}
& M_{{ }_{n}}+M \cdot m \rightarrow M \cdot(n+m) \\
& M \cdot_{n}+M \cdot m \rightarrow M_{n}+M_{m}
\end{aligned}
$$

\subsubsection{Cross-linking}

Polymer molecules can be linked together in various ways, among them random cross-linking which has been studied most frequently (Charlesby 1991). For simple radiation induced cross-linking the number of intermolecular cross-links are proportional to absorbed dose. The formation of each cross-link removes one separate molecule from the population. As the absorbed dose increases the chance of crosslinking occurring between different parts of the same molecules also increases. 


\subsection{Considerations in the choice of phantom}

In radiation physics many different types of solid and liquid have been used to simulate human body tissues. An accurate measurement of the radiation dose distribution in a patient requires a phantom that simulates the patient. The complexity of biological tissues and variations due to factors such as ageing make it difficult to develop a phantom that is suitable for every purpose (Constantinou 1982). Therefore phantom materials should be selected to provide the same attenuation and scattering properties for the specific radiation as the biological tissue. This condition can be met if the materials have the same elemental composition and density as the tissue that is being simulated. In addition specific phantom should be designed and constructed to suit the specific purpose that they are required for. For example recently a semianatomic phantom has been developed (Thwaites and Allahverdi 1997) using epoxy-resin tissue-substitute materials for the purpose of interdepartmental audit. The phantom has been designed to simulate some areas of the human body for treatment, e.g. breast, thorax, head and neck. Apart from these basic requirements, some other important factors that should be taken into account in selecting phantom materials have been given by Wielopolski et al (1985). These are:-

Ease of preparation and handling

Availability of material and cost

Moulding and shaping capacities

Mechanical and chemical stability

Ease of adjusting the composition and density by adding elemental modifiers 
Possibility of introducing detectors

Wielopolski et al (1985) have also stated that "only with gel phantoms can the composition of tissue be exactly duplicated". Therefore the polyacrylamide gel that fulfils most of the above mentioned conditions has been selected as the basic material to simulate tissue (Bini et al 1984). The elemental composition, specific density, and electron density of the polyacrylamide phantom and corresponding muscle tissue is summarised in table 3.1 (Wielopolski et al 1985).

\begin{tabular}{|c|c|c|}
\hline Element & Muscle $^{\mathrm{a}}$ & Soft polyacrylamide \\
\hline \hline $\mathrm{H}$ & 10.20 & 10.18 \\
\hline $\mathrm{C}$ & 12.30 & 12.46 \\
\hline $\mathrm{O}$ & 3.50 & 2.62 \\
\hline $\mathrm{Na}$ & 72.89 & 73.69 \\
\hline $\mathrm{Mg}$ & 0.08 & 0.24 \\
\hline $\mathrm{P}$ & 0.02 & - \\
\hline $\mathrm{S}$ & 0.20 & 0.19 \\
\hline $\mathrm{K}$ & 0.50 & 0.37 \\
\hline $\mathrm{Ca}$ & 0.30 & 0.24 \\
\hline Density $\left(\mathrm{g} / \mathrm{cm}^{3}\right)$ & 1.02 & 1.03 \\
\hline Electron density $\left(10^{23} / \mathrm{cm}^{3}\right)$ & 3.32 & 3.41 \\
\hline
\end{tabular}

a. White 1978

Table 3.1. Elemental composition of muscle and polyacrylamide gel (\% by weight) 


\subsection{Methods and Materials}

\subsubsection{Preparation of $B A N G$ gel}

The BANG gel was manufactured according to the method as described by Maryanski et al (1994). More recently a new method of gel preparation was also introduced by Baldock et al (1998). It is interesting to note that they have commented "although a methodology for the production of PAG (polyacrylamide gel) was described previously (Maryanski et al 1993) some researchers were unable to successfully manufacture PAG".

The gelatin used in the preparation of the BANG gel was type A, from porcine skin, approximately 300 Bloom. The Bloom indicates the strength of the gel. Electrophoresis grade Acrylamide monomer and N,N'-Methylene-bis-Acrylamide cross-linker (bis) (ICN) were used together with deionized water. Oxygen free nitrogen gas was used to remove dissolved oxygen from the gel solution. Oxygen is known to inhibit polymerisation (Bio-rad 1987). The procedures were carried out inside a glove box flushed with nitrogen.

In order to make the required amount of BANG gel, the necessary volume of deionized water needed was added to the glass vessel and deoxygenated by bubbling high purity nitrogen gas through it, at the flow rate of one litre per min for one hour. A concentration of $5 \%$ gelatin (by weight) was added and nitrogen gas was passed over the mixture at the same flow rate. The vessel was subsequently placed in a water bath at $50^{\circ} \mathrm{C}$ and when the gelatin was dissolved the vessel was wrapped in aluminium foil in order to protect the gel from daylight. Then the acrylamide and bis monomers 
(initially $3 \%$ each by weight) were added and the solution was stirred with a magnetic stirrer until the monomers were dissolved.

If the polymer gel solution was to be poured into another vessel for irradiation, that vessel was firstly wrapped in aluminium foil to avoid light induced polymerization of the monomers. It was then flushed with nitrogen gas in order to remove oxygen inside the vessel. The polymer gel solution was then poured into the vessel inside the glove box, while nitrogen was passed through, and was returned to the water bath and bubbled with nitrogen at a flow rate of about $200 \mathrm{cc} \mathrm{min}^{-1}$. Finally after saturating the solution with nitrogen the vessel was placed in ice water with nitrogen gas passing over its surface until the gel solidified.

Acrylamide and bis are reported to be neurotoxic and carcinogenic agents (Hames 1983 and ICN safety information). To avoid any skin and eye contact, inhalation of the vapours, or ingestion the gel was prepared in a well ventilated area, usually in a fume cupboard, and protective clothing worn.

\subsubsection{Effect of oxygen}

The manufacture of the gel was associated with some technical problems. Figure 3.1 shows a photograph of irradiated gel samples given absorbed doses ranging from 0 to $15 \mathrm{~Gy}$. The whole sample was irradiated with a uniform field and the presence of the non-polymerized band below the gel surface indicates the inhibiting effect of oxygen on the free radical polymerization. It can be clearly seen from the figure that the un-polymerized area decreases as the amount of dose increases. It was also observed in preliminary studies that the gel responds less to irradiation when 
contaminated with oxygen. No polymerization was observed up to 4 Gy, with weak polymerization at $5 \mathrm{~Gy}$, although opacity increases as dose increases beyond $5 \mathrm{~Gy}$. Therefore the inhibitive effect of the oxygen seems to be dependant on oxygen concentration in the gel and on the level of the absorbed dose.

Spontaneous polymerization was also observed during the manufacture of some gels. This was caused by prolonged bubbling of nitrogen through the gel in the last step of the manufacturing process. This resulted in increasing fogging of the gel which consequently increased the background $\mathrm{R}_{2}$ value. This problem did not however prevent the gel responding to radiation.

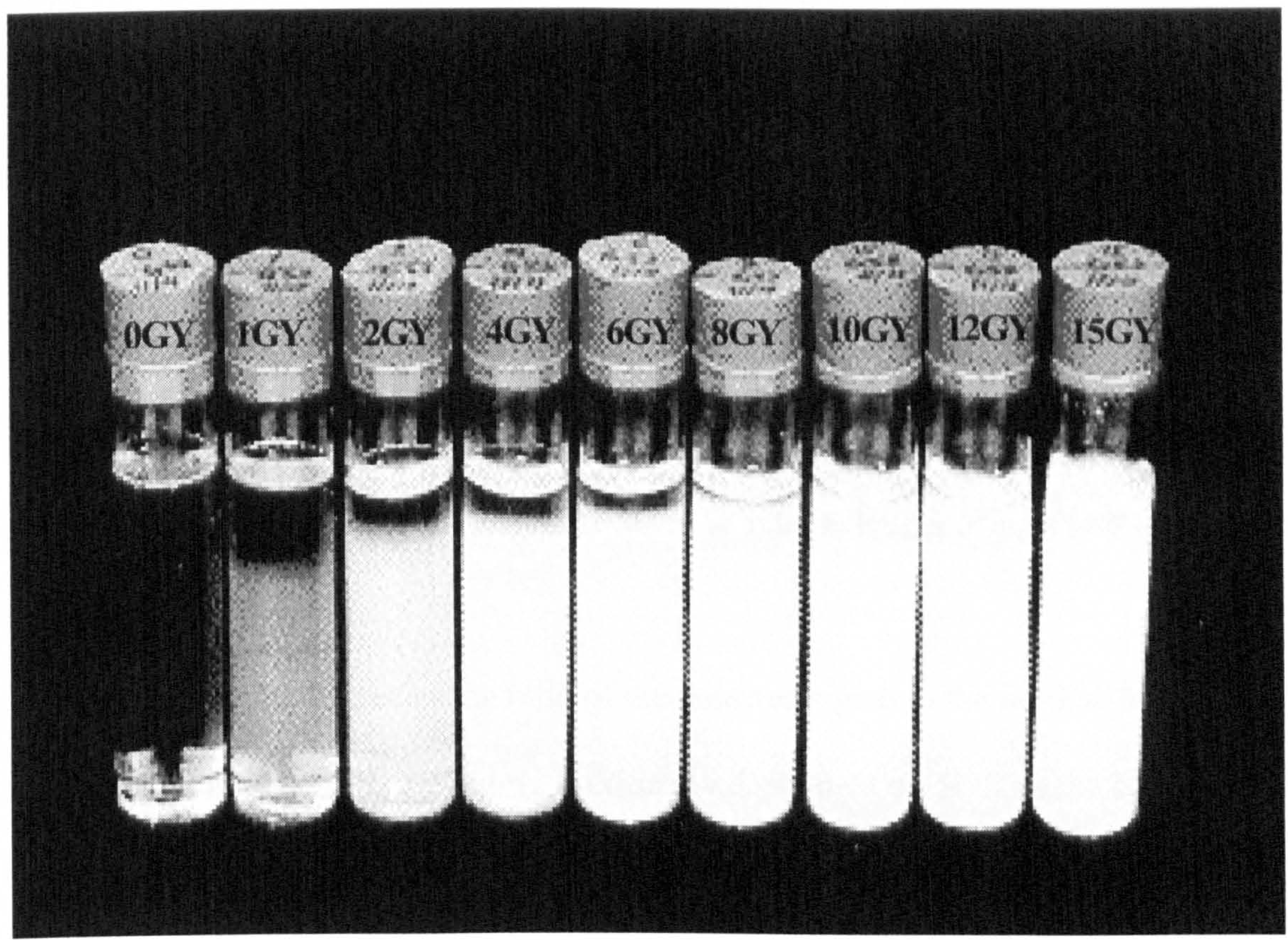

Figure 3.1. Inhibitive effect of the oxygen on the gel response to radiation 


\subsubsection{Measurement of the samples}

The gels were imaged using a 1 Tesla Siemens Impact whole body scanner and head coil using a multi slice spin-echo pulse sequence. The images were then transferred to a Sun Work Station. The transverse relaxation time $\left(T_{2}\right)$ of the water protons in the irradiated gels was then calculated using a programme that employed a two parameter non-linear least square fit to the $\mathrm{T}_{2}$ decay curve.

For the majority of measurements the pulse sequence consisted of a time to repeat (TR) of $6000 \mathrm{~ms}$ with five echo times (TE) of 50, 125, 313, 782, and $2000 \mathrm{~ms}$. The TR was chosen to reduce $T_{1}$-weighting to a minimum, giving a strong MRI signal. However, increasing the TR beyond 6000 would have lead to increased imaging time without much gain in signal intensity. The echo times were chosen to give a good estimation of the $T_{2}$ for the range of $T_{2}$ values that resulted from the irradiated gel. The field of view (FOV) was $190 \mathrm{~mm}$ and the matrix size was $128 \times 256$. The FOV and image matrix were chosen to give adequate image resolution and signal to noise ratio (SNR), since, as the pixel size decreases, so does the SNR. The slice thickness used ranged from 3 to $20 \mathrm{~mm}$. The time taken to image the gel using these parameters was about one hour.

The SNR is defined as the ratio of the detected signal to the random background signal. Magnetic resonance imaging is critically dependent on SNR as the signals from the patient are very weak (Vasila et al 1992). The ratio is a measure of the signal quality and depends on several factors such as field of view, slice thickness and number of excitations. Even though increasing these factors improves the SNR, this is 
at the expense of increasing the time of imaging or poorer spatial resolution. The selection of image parameters is therefore often a compromise.

To determine the magnitude of the improvement in SNR without impairing inplane spatial resolution and also avoiding prolonged scanning time, slice thickness of $3,5,10$, and $15 \mathrm{~mm}$ were used for one particular image while keeping all other imaging parameters constant. Figure 3.2 shows the profiles that were taken through an irradiated gel. It can be seen that the SNR increases as slice thickness increases. Nevertheless, it may be that the gel has inhomogeneity on a pixel scale. However, the gel thought to be homogeneous. To verify this it would be necessary to keep the slice thicknesses constant and improve SNR by signal averaging.
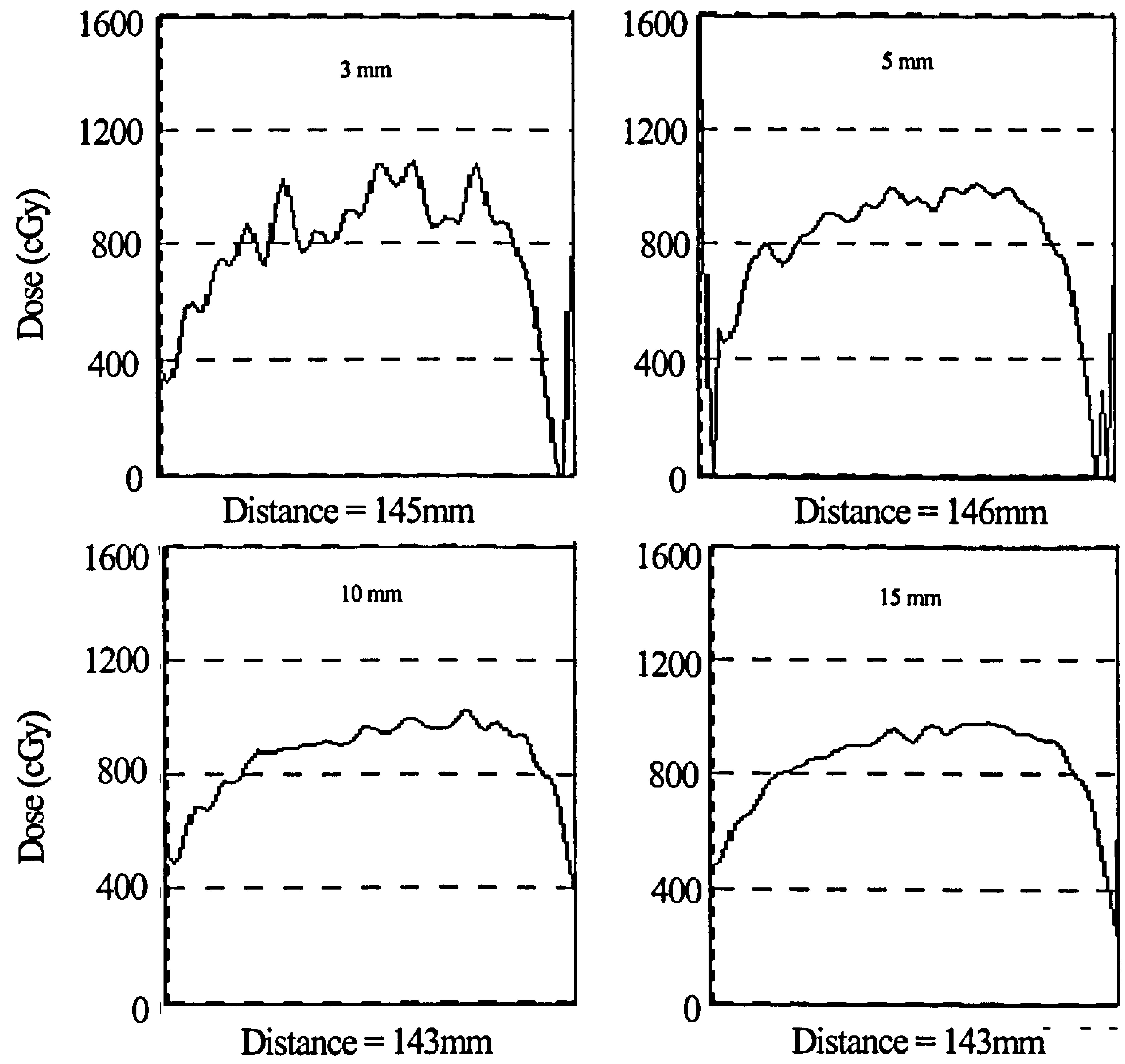

Figure 3.2. Effect of slice thickness on SNR 


\subsection{Basic properties of the BANG gel}

This section addresses the practical aspects of the polymer dosimetry technique, and includes possible sources of error, reproducibility, linearity, and tissue equivalency.

\subsubsection{Sources of error}

The measurements of relaxation time are subject to several sources of error. The main sources of error are the day-to-day variations in scanner performance (variability in coil tuning and pulse flip angle calibration) and the position of the samples inside the magnet.

\subsubsection{Reproducibility of $R_{2}$ measurements}

To determine the reproducibility of relaxation time measurements two main causes of variability in measurements must be taken into account. Firstly the MRI scanner performance, i.e. how constant the scanner performance is from one measurement to another. Secondly variations that may be caused by possible inhomogenities in the gel.

The reproducibility of $R_{2}$ was assessed by repeatedly scanning an irradiated gel sample in a rectangular phantom of $20 \times 12 \times 4.8 \mathrm{~cm}$ and a litre of the un-irradiated gel in a similar phantom. The irradiated samples were scanned six times and the nonirradiated gel three times. The gels were imaged in the centre of the head coil using the same pulse sequence and image parameters as described earlier. The $R_{2}$ values were calculated from the slices that were taken with varying TE values in the same 
region of interest at the centre of the six dose areas. For the un-irradiated gel, the $\mathrm{R}_{2}$ values were calculated in the areas similar to those in the gel. The measurements were made with the samples at room temperature.

Tables 3.2 and 3.3 show the values of $R_{2}$ obtained for gel samples manufactured from the same batch of chemicals. It can be seen from table 3.2 that the $R_{2}$ values in the irradiated gel are reproducible to within a maximum $\pm 4 \%$ of each mean value. It is also evident from table 3.3 that the un-irradiated gel is uniform throughout the phantom with a mean $R_{2}$ value of 1.14 for all positions with a SD of \pm 0.01 . The reproducibility of the MRI scanner can be improved by recalibration of the magnetic field gradient strength on a regular basis and also positioning of the sample in the same location inside the head coil for all the measurements (Horsfield 1998).

\begin{tabular}{|c|c|}
\hline Dose (Gy) & Mean $\mathrm{R}_{2}$ values $\left(\mathrm{s}^{-1}\right)$ \\
\hline 0 & $0.94 \pm 0.03$ \\
\hline 2 & $1.21 \pm 0.05$ \\
\hline 5 & $1.72 \pm 0.04$ \\
\hline 8 & $2.19 \pm 0.05$ \\
\hline 10 & $2.50 \pm 0.04$ \\
\hline 15 & $2.97 \pm 0.09$ \\
\hline
\end{tabular}

Table 3.2. Reproducibility of $R_{2}$ measurements at different doses (quoted uncertainties are $\pm 1 \mathrm{SD}$ ) 


\begin{tabular}{|c|c|}
\hline \hline Location of measurement & $\mathrm{R}_{2}$ values $\left(\mathrm{s}^{-1}\right)$ \\
\hline \hline Top Left & $1.14 \pm 0.02$ \\
\hline Top Right & $1.13 \pm 0.02$ \\
\hline Middle Left & $1.13 \pm 0.02$ \\
\hline Middle Right & $1.14 \pm 0.02$ \\
\hline Bottom Left & $1.13 \pm 0.02$ \\
\hline Bottom Right & $1.16 \pm 0.01$ \\
\hline Mean & $1.14 \pm 0.01$ \\
\hline
\end{tabular}

Table 3.3. $R_{2}$ values obtained in the un-irradiated gel in different location (quoted uncertainties are \pm 1 SD)

\subsubsection{Positional and volume related errors}

The positional and volume related errors are mainly due to the inhomogeneities of the RF pulse which may result in a non-uniform intensity distribution of the image.

To verify how the transverse relaxation time is affected by the position of the gel samples inside the head coil, 9 cylindrical glass vials $24 \mathrm{~mm}$ in diameter and 100 $\mathrm{mm}$ in height were filled with gelatin. The vials were imaged three consecutive times in a rectangular polystyrene box in which they were placed in fixed positions $1 \mathrm{~cm}$ from each other (figure 3.3). The vials were at room temperature. During different measurements the box was in the same location inside the centre of the head coil and vial positions remained unchanged. 
In order to investigate the effect of RF inhomogeniety in a large volume of the gel 2.5 litres of the gel was prepared in a cylindrical phantom of $15 \mathrm{~cm}$ diameter. Then 4 gel vials were irradiated to absorbed doses of 4, 6, 8 and 10 Gy respectively and placed inside the gel (Figure 3.4). The vials were imaged both inside the phantom and outside the phantom in the polystyrene box. The phantom was left in the MRI scanning room before imaging for two days to reach room temperature in order that both images were taken at exactly the same temperature.

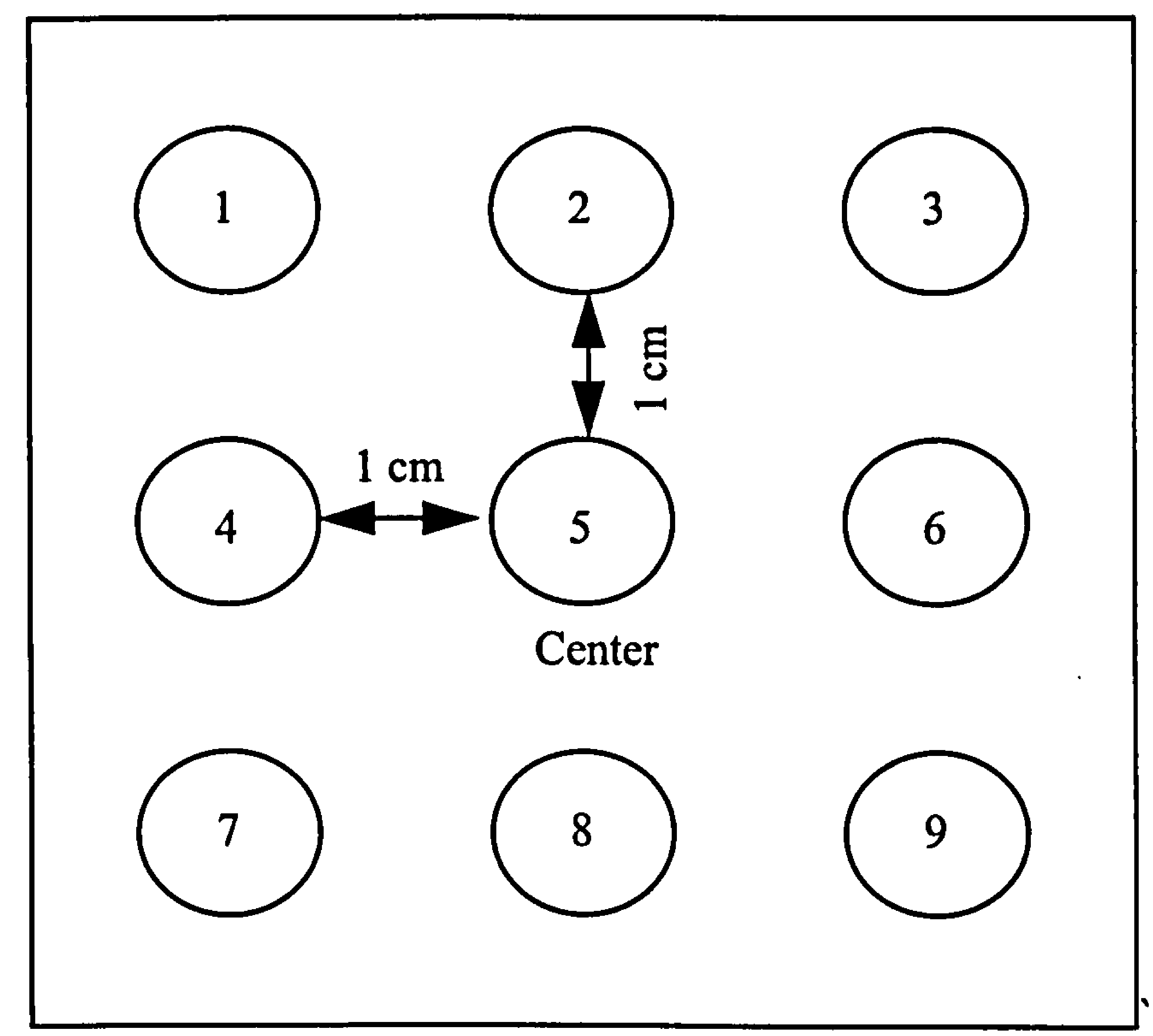

Figure 3.3. Position of the vials inside the head coil where the signals are collected 
The $R_{2}$ values for each vial position were compared by analysis of variance (ANOVA). Table 3.4 shows the mean of the three $R_{2}$ values for each position in the phantom shown in figure 3.3. From the data the mean $R_{2}$ value is 1.20 with a SD of \pm 0.02 for all positions. The statistical comparison between the results obtained for each position showed no significant difference with $\mathrm{P}<0.05$.

Table 3.5 shows the results of $R_{2}$ measurements in the irradiated vial inside and outside of the gel phantom. From the results there is no evidence of any effects due to inhomogeneity in the RF. It should be mentioned that the different dose responses in tables 3.2 and 3.5 are due to the gel being manufactured from different batches of chemicals and also different gel temperatures during MRI imaging.

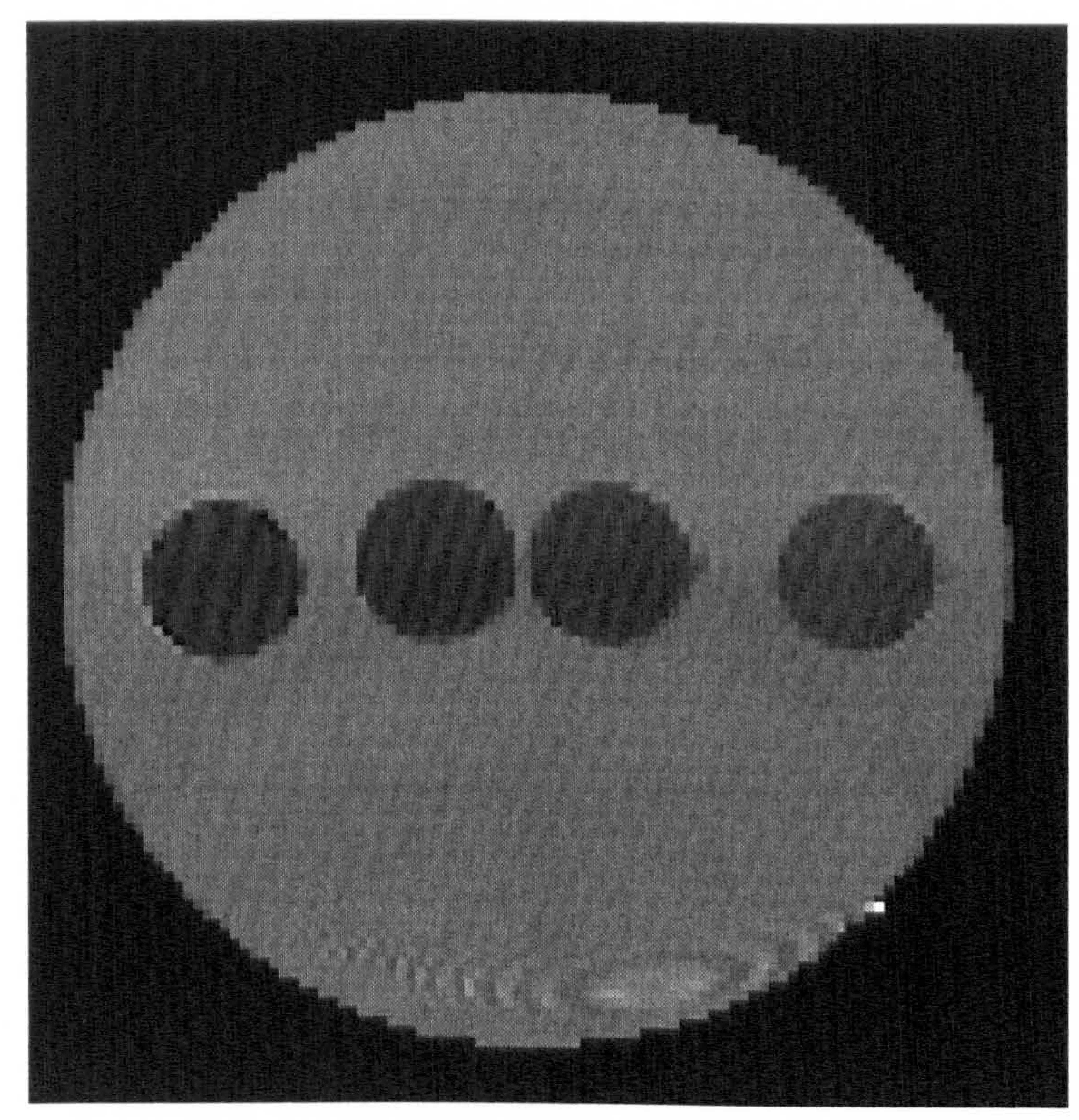

Figure 3.4. $T_{2}$ image of the phantom showing the position of the gel vials inside the phantom 


\begin{tabular}{||c|c|}
\hline Vials position & $\mathrm{R}_{2}\left(\mathrm{~s}^{-1}\right)$ \\
\hline 1 & $1.24 \pm 0.02$ \\
\hline 2 & $1.22 \pm 0.04$ \\
\hline 3 & $1.21 \pm 0.01$ \\
\hline 4 & $1.22 \pm 0.03$ \\
\hline 5 & $1.18 \pm 0.03$ \\
\hline 6 & $1.19 \pm 0.00$ \\
\hline 7 & $1.20 \pm 0.02$ \\
\hline 8 & $1.18 \pm 0.04$ \\
\hline 9 & $1.20 \pm 0.01$ \\
\hline Mean & $1.20 \pm 0.02$ \\
\hline
\end{tabular}

Table 3.4. $R_{2}$ values obtained at different positions (quoted uncertainties are $\pm 1 \mathrm{SD}$ )

\begin{tabular}{|c|c|c|}
\hline Dose (Gy) & $\begin{array}{c}\mathrm{R}_{2} \text { measured inside } \\
\text { the gel phantom }\end{array}$ & $\begin{array}{l}\mathrm{R}_{2} \text { measured inside } \\
\text { the polystyrene box }\end{array}$ \\
\hline 4 & $2.43 \pm 0.03$ & $2.48 \pm 0.1$ \\
\hline 6 & $2.83 \pm 0.1$ & $2.83 \pm 0.1$ \\
\hline 8 & $3.37 \pm 0.06$ & $3.36 \pm 0.16$ \\
\hline 10 & $3.54 \pm 0.1$ & $3.57 \pm 0.08$ \\
\hline
\end{tabular}

Table 3.5. $R_{2}$ values measured in the gel vials imaged inside the gel phantom and polystyrene box 


\subsubsection{Reproducibility of the dose response}

In order to investigate the reproducibility of the absorbed dose response, three BANG gels, each of volume one litre were manufactured from the same batch of chemicals as described previously (see 3.6.1). The gels were irradiated in a rectangular Perspex box measuring $20 \times 12 \times 4.8 \mathrm{~cm}$ with wall thickness of $2.5 \mathrm{~mm}$ on the same day as they were prepared. This was repeated using chemicals from different batches. The front and back surfaces of the box were inscribed with six $4 \times 4 \mathrm{~cm}$ regions corresponding to radiation fields; the centre of the fields were also marked on the box. Prior to gel irradiation the dose to the centre of each area was determined using a calibrated PTW Unidos electrometer and NE 2581 ion chamber. The calibration measurements were made inside an identical but open Perspex box containing the gelatin only (Figure 3.5).

Each gel was irradiated using $6 \mathrm{MV}$ X-rays from a Philips SL 75/5 linear accelerator. The dimensions of the irradiation field were $4 \times 4 \mathrm{~cm}$ and the source to surface distance (SSD) was $100 \mathrm{~cm}$. In order to give sufficient build-up and adequate backscatter $2 \mathrm{~cm}$ of Perspex was placed in front and $4 \mathrm{~cm}$ behind the gel container. Gel I was irradiated to absorbed doses of $0,1,2,3,4, \& 5$ Gy, gel II to $0,2,4,6,8, \& 10$ Gy and gel III to $0,2,5,810, \& 15$ Gy. In order to give a uniform dose distribution through the width of the irradiated area, all the doses were delivered in two equal parts, using parallel opposed fields. After irradiation the gels were stored in a refrigerator.

MR images of the gels were made five days after irradiation. The gel temperature was the same for all measurements (nominal $4^{\circ} \mathrm{C}$ ). A $3 \mathrm{~mm}$ thick transverse slice (parallel to the irradiated surface) was taken through the middle of the 
gel. The transverse relaxation rates $\left(\mathrm{R}_{2}=\mathrm{T}_{2}{ }^{-1}\right)$ were calculated from an area $1.2 \times 1.2$ $\mathrm{cm}$ at the centre of the radiation field for each of the five spin-echo images.

The polymerized area becomes visible at a dose of approximately 1 Gy and its opacity can be seen qualitatively to increase with dose as shown in figure 3.6.

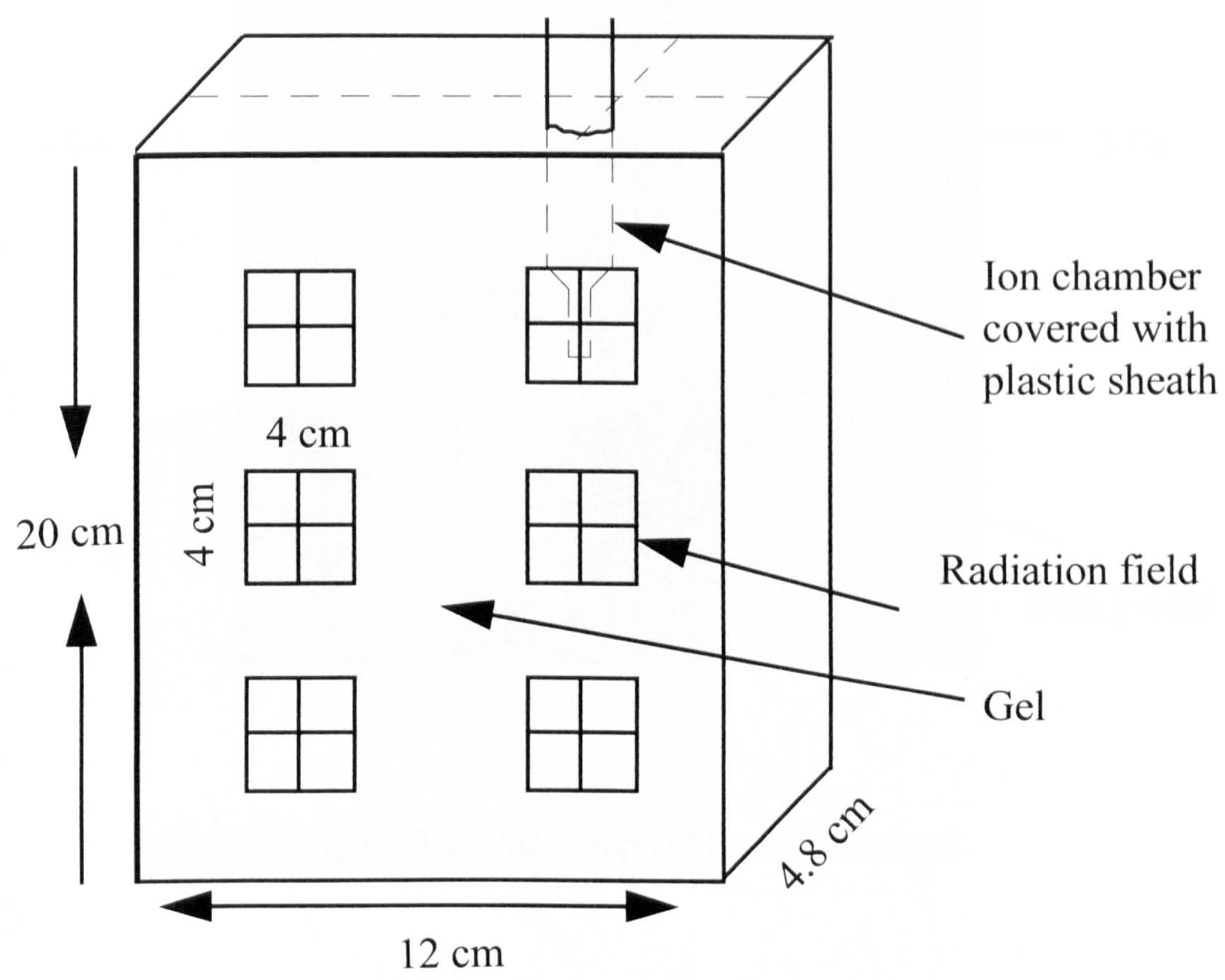

Figure 3.5. Set up for radiation beam calibration 


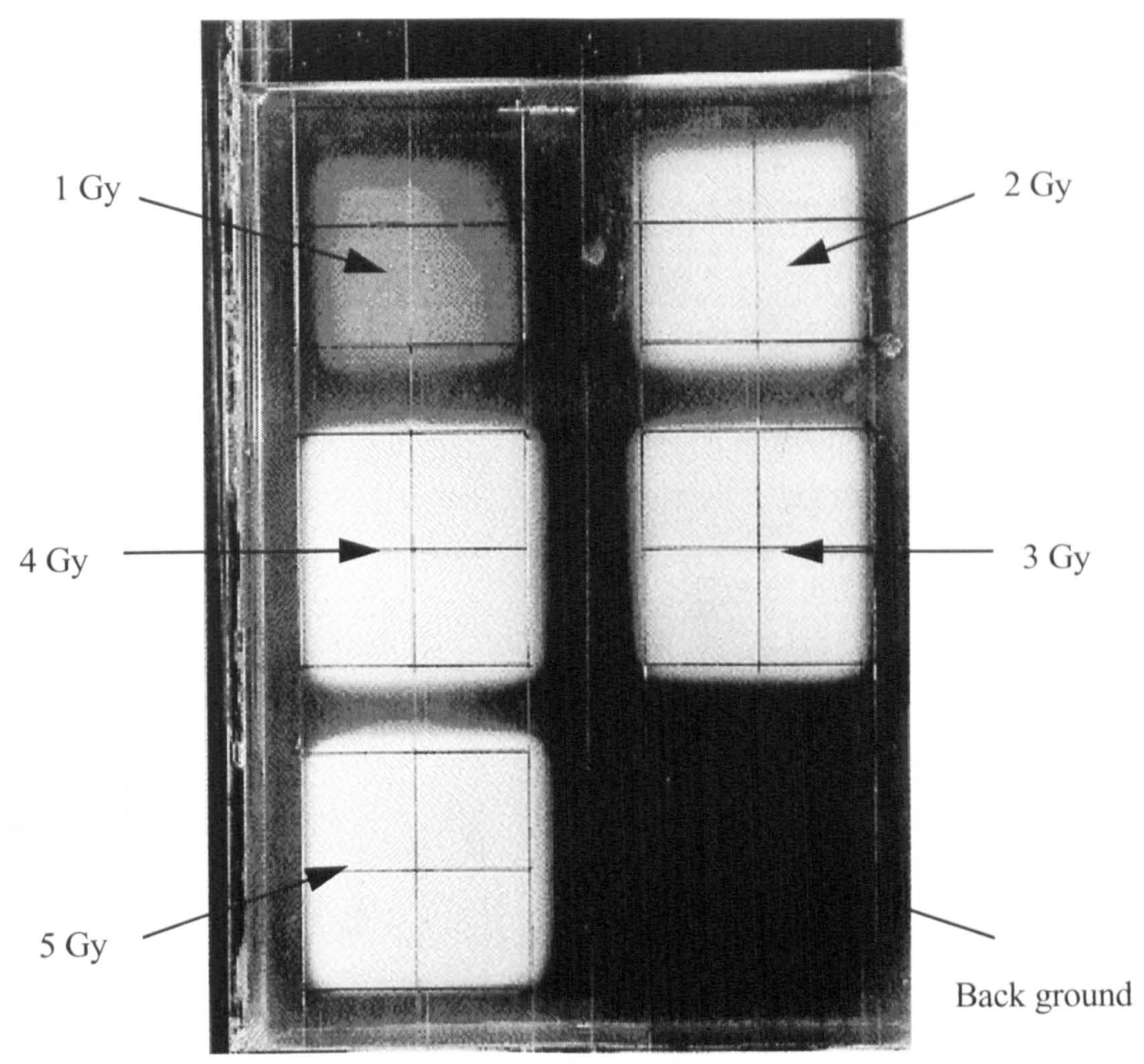

Figure 3.6. Photograph of the irradiated gel

Figure 3.7 shows the results obtained from relaxation rate measurements $\left(R_{2}\right)$ in three different sets of the samples, prepared on different days. The data in figure 3.7 shows that the dose response is highly reproducible over the range of the measured dose within $\pm 4 \%$ provided that chemicals are taken from the same batch. Figure 3.8 shows the best fit line for each individual gel. The limit of linearity of dose response in this case was 10 Gy as can be seen from figure 3.8. Later on with subsequent measurements linearity was found up to 12 Gy (figure 3.9). 
The results for samples manufactured from different batches are shown in figure 3.10. It can be seen that different batches of the chemical resulted in differences in the dose response. The difference in slope between the two batches is $23 \%$.

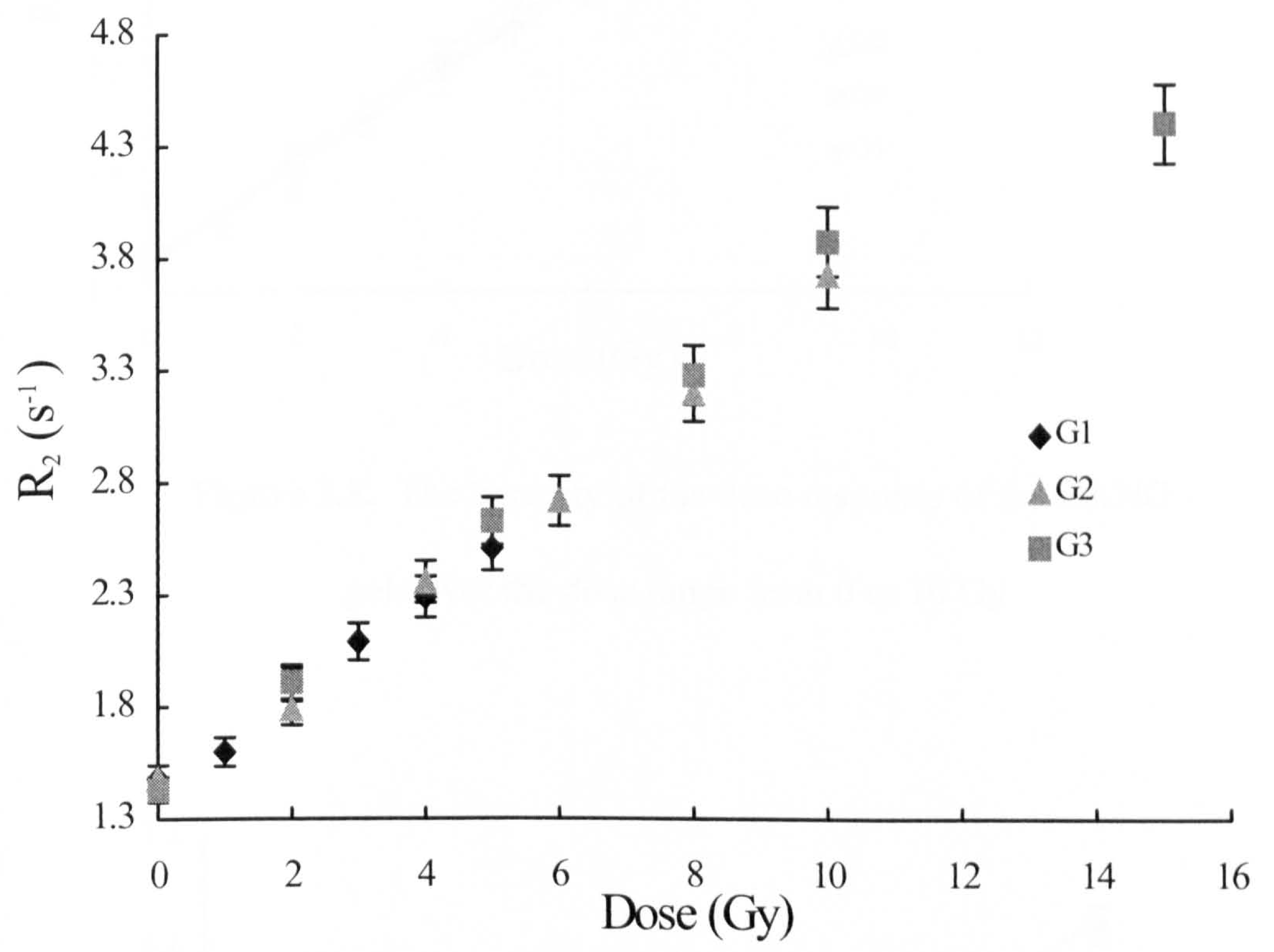

Figure 3.7. The dependence of the transverse relaxation rate, $R_{2}$ on dose in three separately prepared gels over the range from 0 to $15 \mathrm{~Gy}$. 


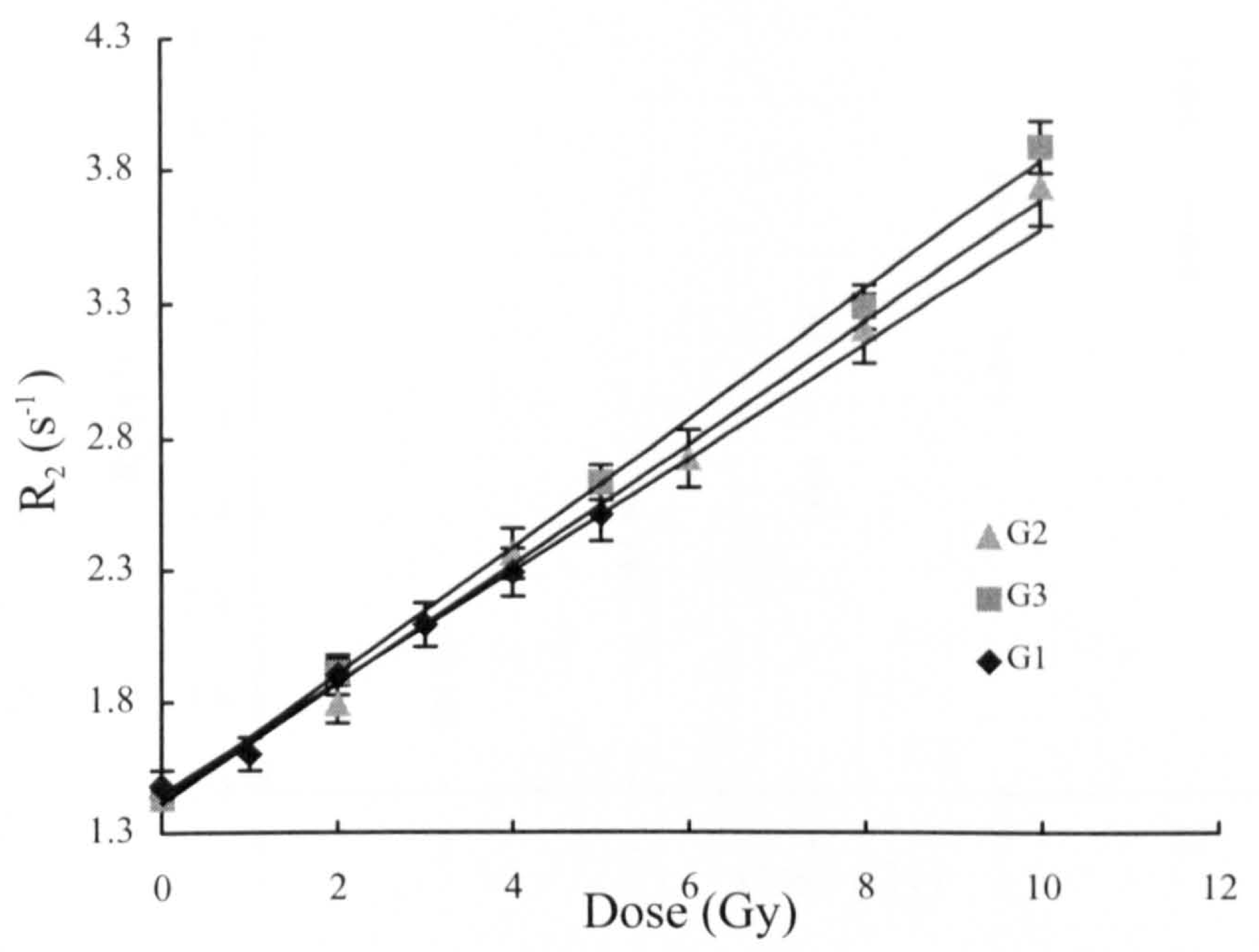

Figure 3.8. The linearity of the dose response of the BANG gels over the dose range from 0 to $10 \mathrm{~Gy}$.

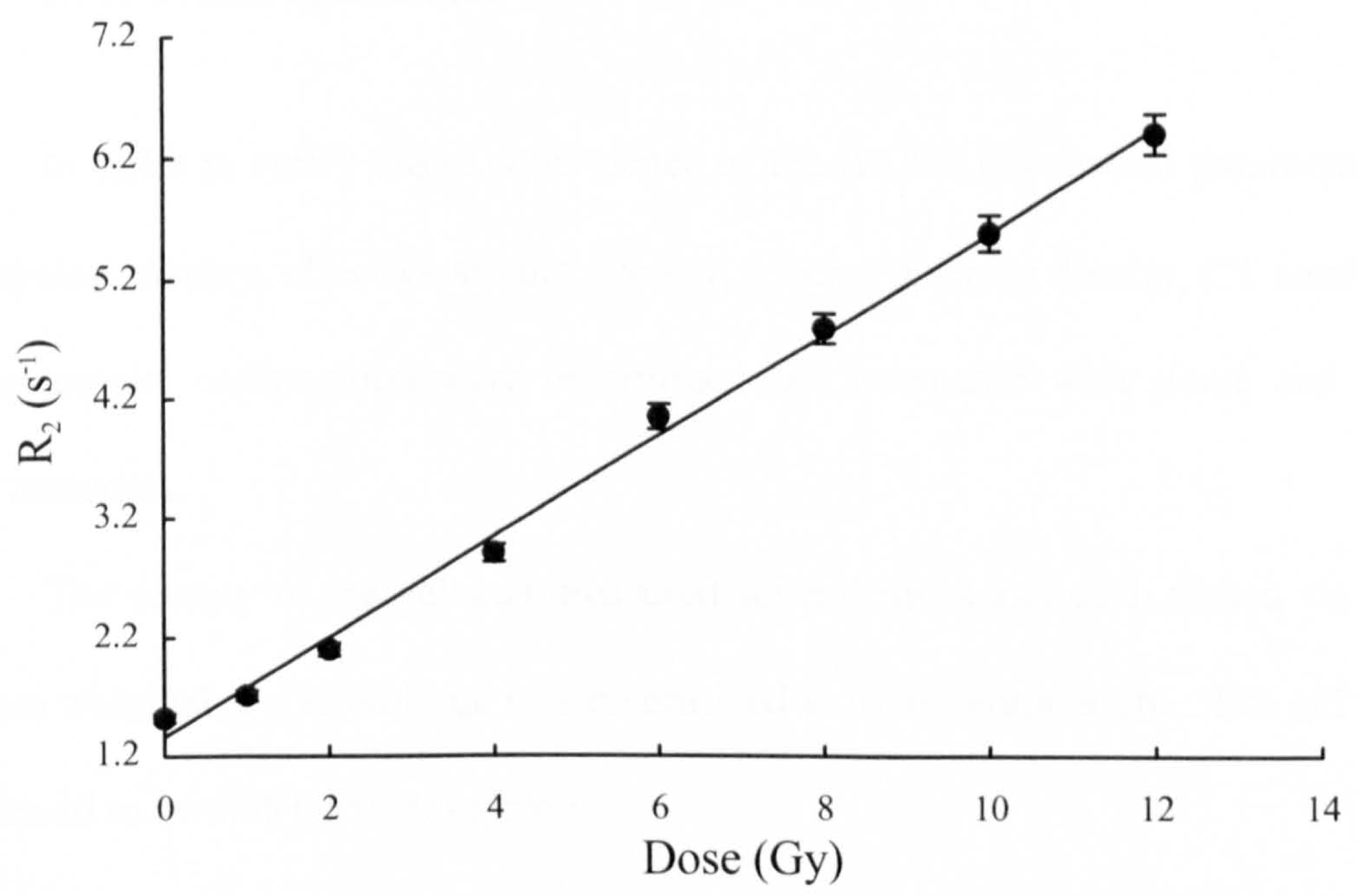

Figure 3. 9. Linearity of the $\mathrm{R}_{2}$ measurements to dose up to $12 \mathrm{~Gy}$ 


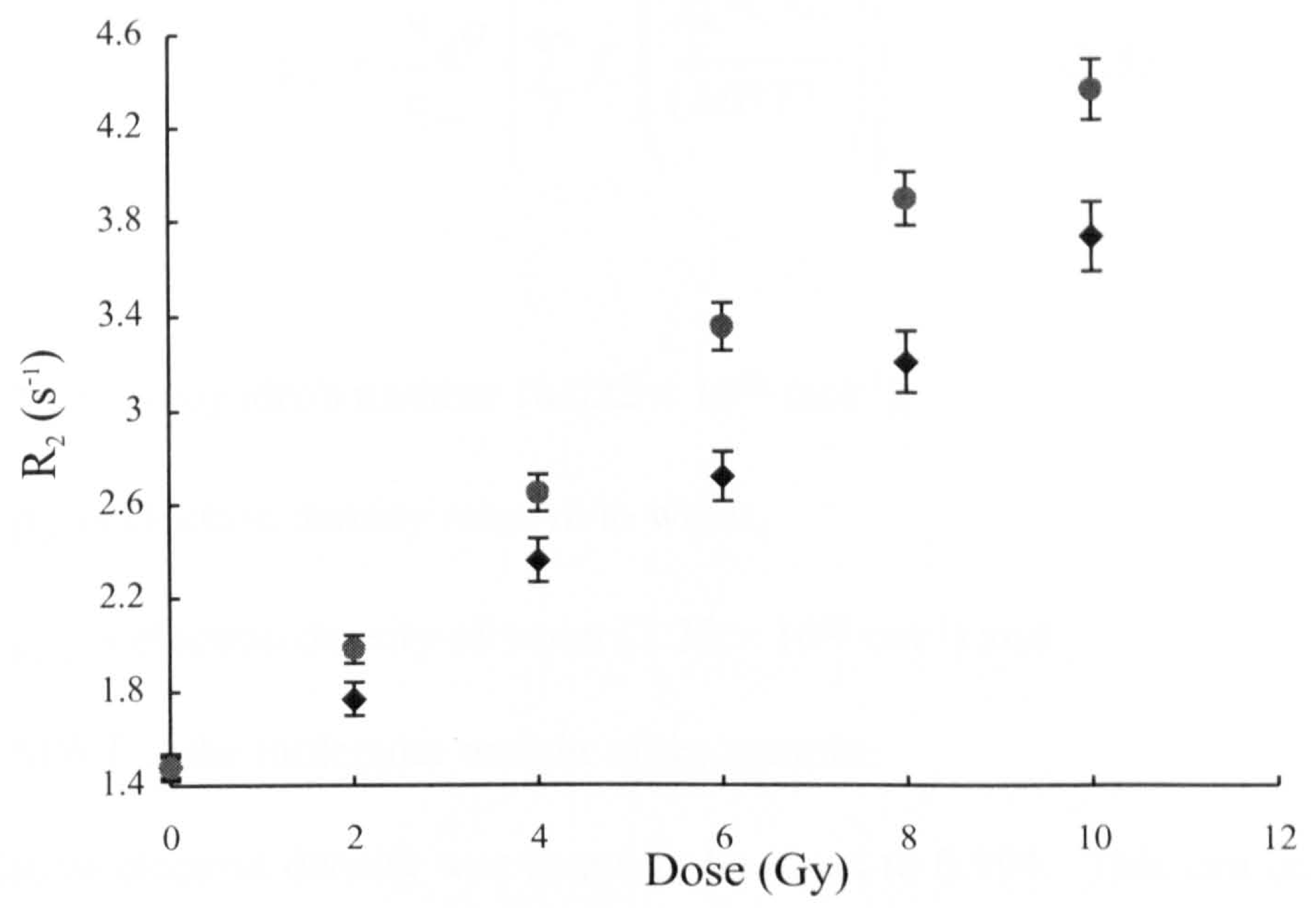

Figure 3.10. Effect of the different chemical batch

on the gel response

\subsubsection{Tissue equivalence}

In order to verify tissue equivalence of the BANG gel several parameters such as, physical density, effective atomic number, relative electron density, CT number and also elemental composition were determined and compared with tissue and several other materials.

The density of the gel was measured several times and each time a vial of the gel was weighed and its volume was determined at room temperature. The gel density was found to be $0.991 \pm 0.006 \mathrm{~g} \mathrm{~cm}^{-3}$.

From the sum of mass fraction $f_{j}$ of the various molecular components of the gel and their elemental atomic number $z_{i}$ the relative electron density $\left(\rho_{e}^{w}\right)$ was calculated using the formula given by McCullough and Holmes (1985) 


$$
\rho_{e}^{w}=\frac{N_{A} \rho}{\rho_{e w}}\left\{\sum_{j} f_{j} \cdot\left(\frac{\sum_{i} n_{t, j} z_{1, j}}{(M W T)_{1}}\right)\right\}
$$

where,

$\mathrm{N}_{\mathrm{A}}$ is Avogadro's number $\left(6.022 \times 10^{23} \mathrm{~mol}^{-1}\right)$,

$\rho_{e}^{w}$ is electron density relative to water,

$\rho_{\mathrm{ew}}$ is electron density of water $\left(3.33 \times 10^{23} \mathrm{~cm}^{-1}\right)$ and

MWT is the molecular weight of the sample

The relative electron density was found to be equal to 0.994 . This can be also derived directly from the CT number and depends on the effective atomic number $\left(Z_{\text {eff }}\right)$. For water-like material $\left(Z_{\text {eff }} \sim 7.4\right)$ this can be computed from the following equation (Battista et al 1980);

$$
\rho_{e}^{w}=1+\frac{\text { CT number }}{1000}
$$

For a material where the $\mathrm{Z}_{\text {eff }}$ is considerably different from that of water the equation is;

$$
\rho_{e}^{w}=1.052+\frac{4.8 \times \mathrm{CT} \text { number }}{10000}
$$

The accuracy of $\rho_{e}^{w}$ for most of tissues using this formula is within \pm 0.03 (Battista et al 1980).

To find the CT number a sample of the non-irradiated gel was imaged using Somatom CT scanner and CT number was measured. The CT number of BANG gel was $17.7 \pm 3.2$. By substituting the value of $\mathrm{CT}$ number in equation 3.6 the relative electron density was found to be 1.02 . 
The effective atomic number was calculated using the expression (McCullough and Holmes 1985)

$$
Z_{e f f}=\left[\frac{N_{A} \rho}{\rho_{e}^{w}}\right]^{1 / a} \cdot\left\{\sum_{j} \frac{f_{j}}{(M W T) j} \cdot\left[\sum_{i} n_{i, j}\left(z_{i, j}\right)^{a+1}\right]\right\}^{1 / a}
$$

Using the value of $\rho_{e}^{w}$ calculated from equation (3.5) $\mathrm{Z}_{\text {eff }}$ was found equal to 7.33. The value of "a" used was 2.94 as suggested by McCullough and Holmes (1985).

The elemental composition of BANG gel is given in table 3.6 and the effective atomic number, CT number and physical and electron density in table 3.7 together with data for some other materials. It can be seen that effective atomic number, relative electron density and physical density of the BANG polymer gel is close to the water.

\begin{tabular}{|c|c|c|c|c|c|c|c||}
\hline Element & $\mathrm{Z}$ & $\mathrm{A}$ & Fricke $^{\mathrm{a}}$ & BANANA $^{\mathrm{S}}$ & SDA gel $^{\mathrm{N}}$ & Gelatin gel $^{\mathrm{b}}$ & BANG \\
\hline \hline $\mathrm{H}$ & 1 & 1.008 & 11.077 & 10.781 & 11.049 & 10.763 & 10.647 \\
\hline $\mathrm{C}$ & 6 & 12.01 & 0.578 & 3.967 & 0.691 & 1.959 & 5.262 \\
\hline $\mathrm{N}$ & 7 & 14.01 & 0.0041 & 1.282 & 0.0014 & 0.665 & 1.852 \\
\hline $\mathrm{O}$ & 8 & 16.00 & 88.160 & 83.372 & 88.029 & 85.757 & 82.229 \\
\hline $\mathrm{Na}$ & 11 & 22.99 & 0.0023 & - & - & 0.0021 & - \\
\hline $\mathrm{S}$ & 16 & 32.06 & 0.167 & - & 0.227 & 0.847 & - \\
\hline $\mathrm{Cl}$ & 17 & 35.45 & 0.0035 & - & - & 0.0033 & - \\
\hline $\mathrm{Ca}$ & 20 & 40.08 & - & - & - & - & - \\
\hline $\mathrm{Fe}$ & 26 & 55.85 & 0.0055 & - & 0.0027 & 0.0026 & \\
\hline
\end{tabular}

a Djennaui (1994)

b Kron et al (1993)

Table 3.6. Elemental composition (\% by weight) of dosimetry gels 


\begin{tabular}{|c|c|c|c|c|}
\hline Substance & $\overline{\rho_{e}^{w}}$ & $\rho$ & $\mathrm{N}_{\mathrm{CT}}$ & $\mathrm{Z}_{\mathrm{eff}}$ \\
\hline Water & 1 & 1 & 0 & 7.42 \\
\hline Muscle $^{a}$ & 0.94 & $\overline{1}$ & - & 7.61 \\
\hline$\overline{\text { Boneb }}$ & 1.1 & 1.12 & 236 & 9.32 \\
\hline$\overline{\text { BANG }}$ & 0.994 & 0.991 & 17.7 & 7.33 \\
\hline Gelatin gelc & 1.004 & 1.005 & 10 & 7.56 \\
\hline SDA gelc & 1.006 & $1.005 \pm 0.005$ & $8 \pm 5$ & 7.46 \\
\hline BANANA $^{d}$ & 1.005 & 1.012 & 13.4 & 7.36 \\
\hline Fricke $^{d}$ & 1.004 & 1.003 & 10.8 & 7.45 \\
\hline & & d I & 19 & \\
\hline
\end{tabular}

Table 3.7. Physical quantities of various substance relevant to gel dosimetry

\subsubsection{Effect of monomers and gelatin concentration}

To investigate the role of total monomers (acrylamide and bis-acrylamide), and gelatin concentration on the dose response 6 BANG gels each of $500 \mathrm{ml}$ were prepared using chemicals from the same batches. Three of the gels had a different concentration of monomers, namely $6,8, \& 9 \%$ by weight (equal amounts of acrylamide and bis) but the same amount of gelatin (5\%). A further gel was made using $4 \%$ acrylamide $3 \%$ bis and $5 \%$ gelatin, another using $3 \%$ acrylamide $4 \%$ bis $5 \%$ gelatin, and finally one $3 \%$ acrylamide $3 \%$ bis $6 \%$ gelatin. All the gel solutions were made using glass bottles. When the solution was ready, 9 glass vials of $100 \mathrm{~mm}$ height, $24 \mathrm{~mm}$ diameter were filled and placed in the ice-water to solidify. Before pouring the mixture, the vials 
were flushed with nitrogen to remove oxygen and covered in an aluminium foil to protect from daylight.

The vials were irradiated in a phantom using $6 \mathrm{MV}$ x-rays. The beam was calibrated using an ion chamber. The chamber was placed in water substitute epoxy resin, WT1 (White 1977, Constantinou 1978) that was shaped to fit into a glass vial. The vial was then placed in the centre of a purpose made Perspex phantom (Figure 3.11). The phantom was irradiated using a field of $15 \times 15 \mathrm{~cm}$ with the phantom surface placed at an SSD of $100 \mathrm{~cm}$. After calibration the vial with the ion chamber was replaced with the one containing the gel. Each set of the vials was irradiated to absorbed doses of $0,1,2,4,6,8,10,12$, and $15 \mathrm{~Gy}$. In order to give a uniform dose and avoid dose gradients across the vials, the total dose was given in two equal parts from each side. The vials were then stored in a refrigerator.

The $\mathrm{R}_{2}$ measurements were performed one week after radiation. The vials were scanned in a polystyrene box (see 3.7.1.2). A $3 \mathrm{~mm}$ thick coronal slice was taken and $R_{2}$ was measured from $1.2 \times 1.2 \mathrm{~cm}$ at the centre of the radiation field for each of the five spin-echo images. The gel temperature for all measurements was $4^{\circ} \mathrm{C}$ unless otherwise indicated.

Figure 3.12 illustrates the dependence of the dose sensitivity on the total amount of monomer (acrylamide and bis). It can be seen that the sensitivity (slope of dose response curve) increases as the amount of total monomer increases. The slope of dose response varies from $0.28 \pm 0.01 \mathrm{~s}^{-1} \mathrm{~Gy}^{-1}$ for a total monomer concentration of 6 $\%$ to $0.5 \pm 0.02 \mathrm{~s}^{-1} \mathrm{~Gy}^{-1}$ for $9 \%$ as shown in table 3.8. It was also found that the gel samples which were prepared with $9 \%$ of the total monomer produced a crystalline salt on the top of the samples. 


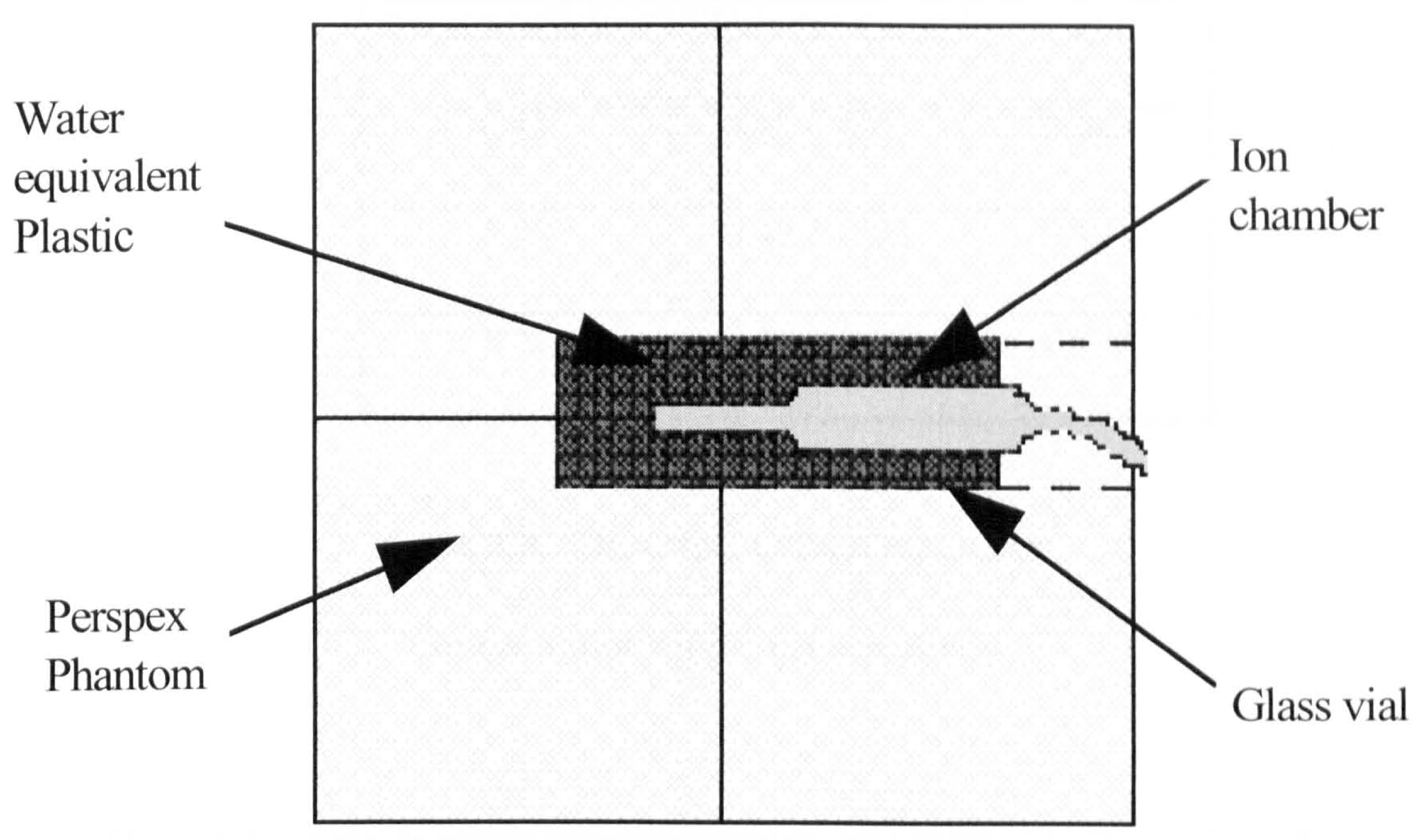

Figure 3.11. Diagram showing set up for beam calibration

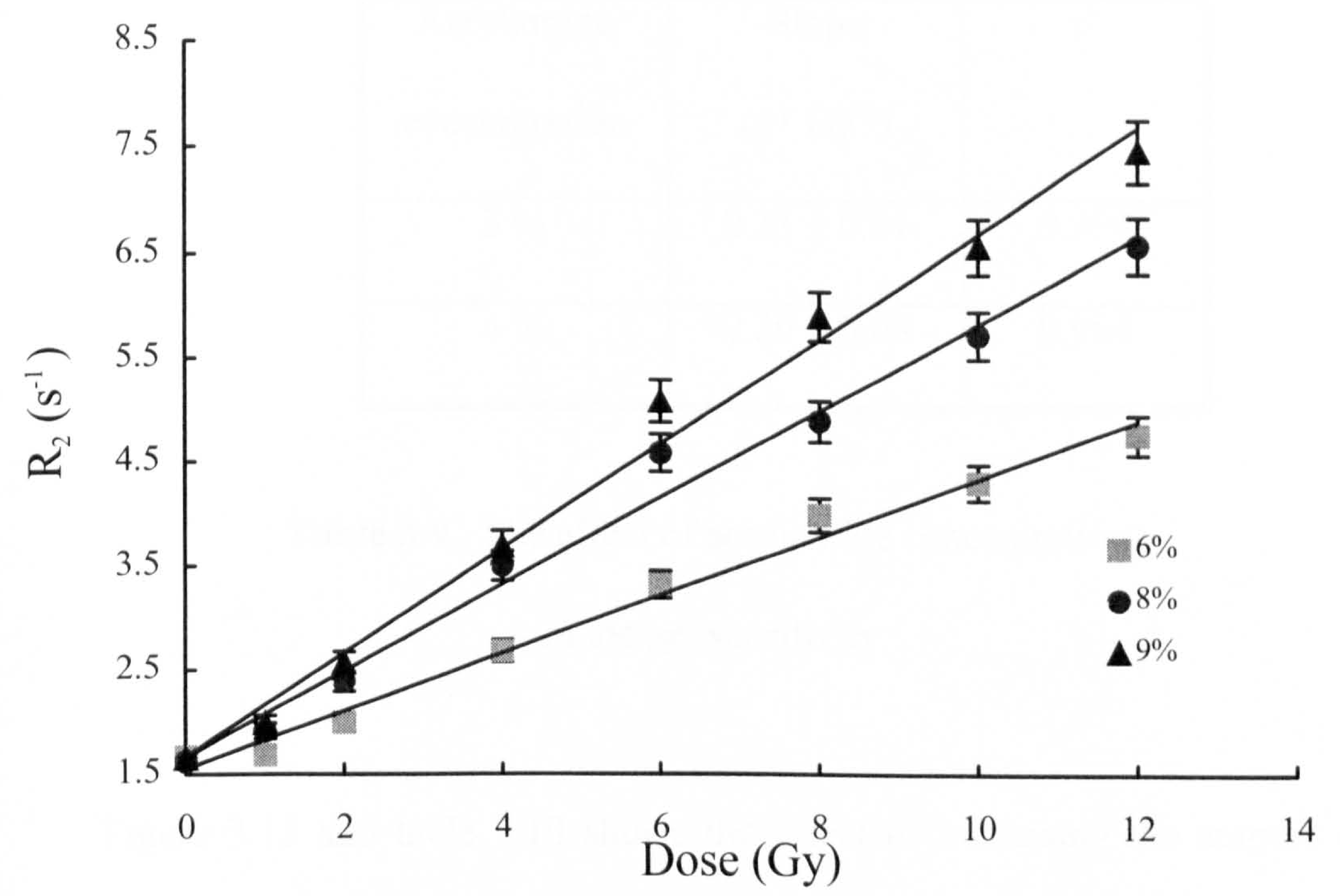

Figure 3.12. Dose response curves obtained with different total monomer concentration. 


\begin{tabular}{|c|c|c|}
\hline \hline Total Monomer & Slope $\left(\mathrm{s}^{-1} \mathrm{~Gy}^{-1}\right)$ & $\mathrm{r}^{2}$ \\
\hline $6 \%$ & $0.28 \pm 0.01$ & 0.994 \\
\hline $8 \%$ & $0.42 \pm 0.02$ & 0.994 \\
\hline $9 \%$ & $0.50 \pm 0.02$ & 0.995 \\
\hline
\end{tabular}

Table 3.8. The effect of total monomer concentration on the gel sensitivity

The result of the increase in concentration of acrylamide alone, from $3 \%$ to 4 $\%$ is shown in table 3.9. It can be seen that there is no difference in slope between 3 and $4 \%$ of acrylamide.

\begin{tabular}{|c|c|c|}
\hline $\begin{array}{c}\text { Acrylamide } \\
\text { concentration }\end{array}$ & $\begin{array}{c}\text { Slope } \\
\left(\mathrm{s}^{-1} \mathrm{~Gy}^{-1}\right)\end{array}$ & \\
\hline $3 \%$ & $0.28 \pm 0.01$ & 0.994 \\
\hline $4 \%$ & $0.29 \pm 0.03$ & 0.964 \\
\hline
\end{tabular}

Table 3.9. The effect of acrylamide concentration on the gel sensitivity

Figure 3.13 and table 3.10 shows the effect of increasing the amount of bis alone on dose response of the gel. From the table, the slope of the gel response is not affected by increasing the bis concentration from 3 to $4 \%$. However the limit of dose 
linearity was found to increase from $12 \mathrm{~Gy}$ to $15 \mathrm{~Gy}$ as the amount of bis increases from 3 to $4 \%$ as shown in figure 3.13 .

\begin{tabular}{||c|c|c||}
\hline Bis-acrylamide & Slope & $\mathrm{r}^{2}$ \\
concentration & $\left(\mathrm{s}^{-1} \mathrm{~Gy}^{-1}\right)$ & \\
\hline \hline $3 \%$ & $0.28 \pm 0.01$ & 0.994 \\
\hline $4 \%$ & $0.29 \pm 0.01$ & 0.996 \\
\hline
\end{tabular}

Table 3.10. The effect of bis-acrylamide concentration on the gel sensitivity

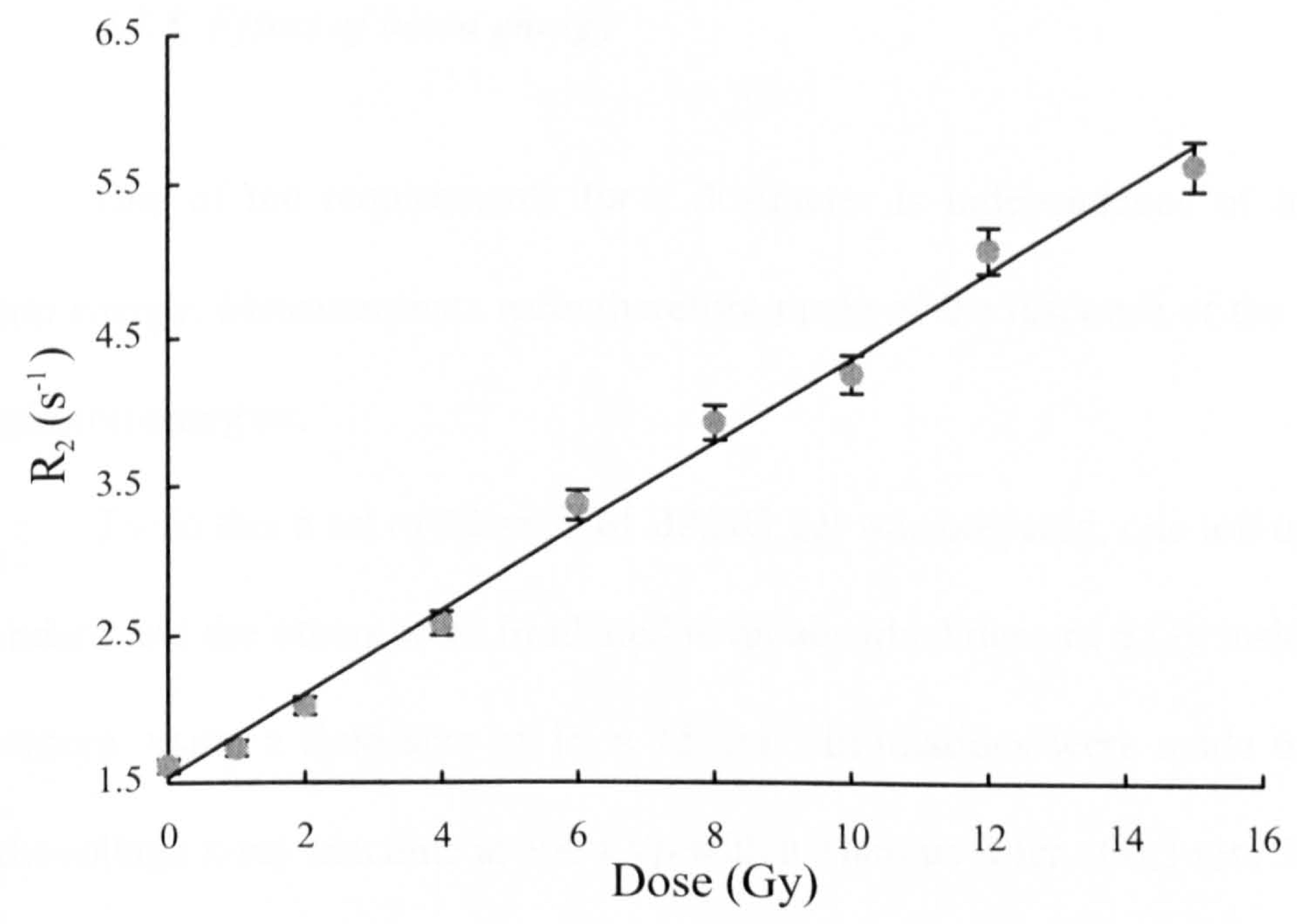

Figure 3.13. Dose response curve obtained from $\mathrm{R}_{2}$ measurement in the gel with $4 \%$ of bis 
Increasing the amount of gelatin from 5 to $6 \%$ resulted in a slight decrease in the slope of the $R_{2}$ dose response of the gel from 0.28 to $0.26 \mathrm{~s}^{-1} \mathrm{~Gy}^{-1}$ respectively (table 3.11).

\begin{tabular}{|c|c|c|}
\hline $\begin{array}{c}\text { Gelatin } \\
\text { concentration }\end{array}$ & $\begin{array}{c}\text { Slope } \\
\left(\mathrm{s}^{-1} \mathrm{~Gy}^{-1}\right)\end{array}$ & \\
\hline \hline $5 \%$ & $0.28 \pm 0.01$ & 0.994 \\
\hline $6 \%$ & $0.26 \pm 0.01$ & 0.993 \\
\hline
\end{tabular}

Table 3.11. The effect of gelatin concentration on the gel sensitivity

\subsubsection{Effect of beam energy}

One of the requirements for a dosimeter is independence of its response to beam energy. Measurements were therefore made of the response of the gel to a range of photon energies.

To do this a set of 12 vials of BANG gel was prepared, one left unexposed as a standard and the others were irradiated to an absorbed dose of $5 \mathrm{~Gy}$ inside the Perspex phantom, using a field size of $15 \times 15 \mathrm{~cm}$. Irradiations were made using a Pantak orthovoltage $\mathrm{x}$-ray machine at $300 \mathrm{kVp}$ with a Thoreus filter of $0.3 \mathrm{~mm} \mathrm{Sn}, 0.5 \mathrm{~mm} \mathrm{Cu}$ and $1.5 \mathrm{~mm} \mathrm{Al}$ (3 vials), $\mathrm{Co}^{60}$ (3 vials), a Philips $6 \mathrm{MV}$ SL 75/5 (2 vials), and an $8 \mathrm{MV}$ Philips SL 75/20 (3 vials). Each beam was calibrated prior to irradiation using the 
appropriate IPEM code of practice. For the Pantak the IPEMB 1996 was used and for the other beams IPSM 1990. The gels imaged as described previously (see 3.7.4).

The results of $R_{2}$ measurements for different beam energies are shown in table 3.12. It can be seen that the dose response of the BANG gel is independent of the energy to within less than $\pm 5 \%$.

\begin{tabular}{|c|c|}
\hline Beam energy & Gel response $\mathrm{R}_{2}\left(\mathrm{~s}^{-1}\right)$ \\
\hline \hline $300 \mathrm{kVp}$ & $1.68 \pm 0.08$ \\
\hline $\mathrm{CO}^{60}($ nominal $4 \mathrm{MV})$ & $1.69 \pm 0.08$ \\
\hline $6 \mathrm{MV}$ & $1.76 \pm 0.08$ \\
\hline $8 \mathrm{MV}$ & $1.68 \pm 0.08$ \\
\hline
\end{tabular}

Table 3.12. Variation of the BANG gel response with energy

To confirm the result and to investigate the difference in gel response to continuous and pulsed radiation, 10 vials of the gel were irradiated to absorbed doses of $0,2,4,6$, and 8 , Gy produced from the $\mathrm{Co}^{60}$ unit and the $6 \mathrm{MV}$ linear accelerator. The results are shown in figure 3.14 and no difference in the gel response to the continuous and pulsed radiation can be observed to within $\pm 5 \%$. 


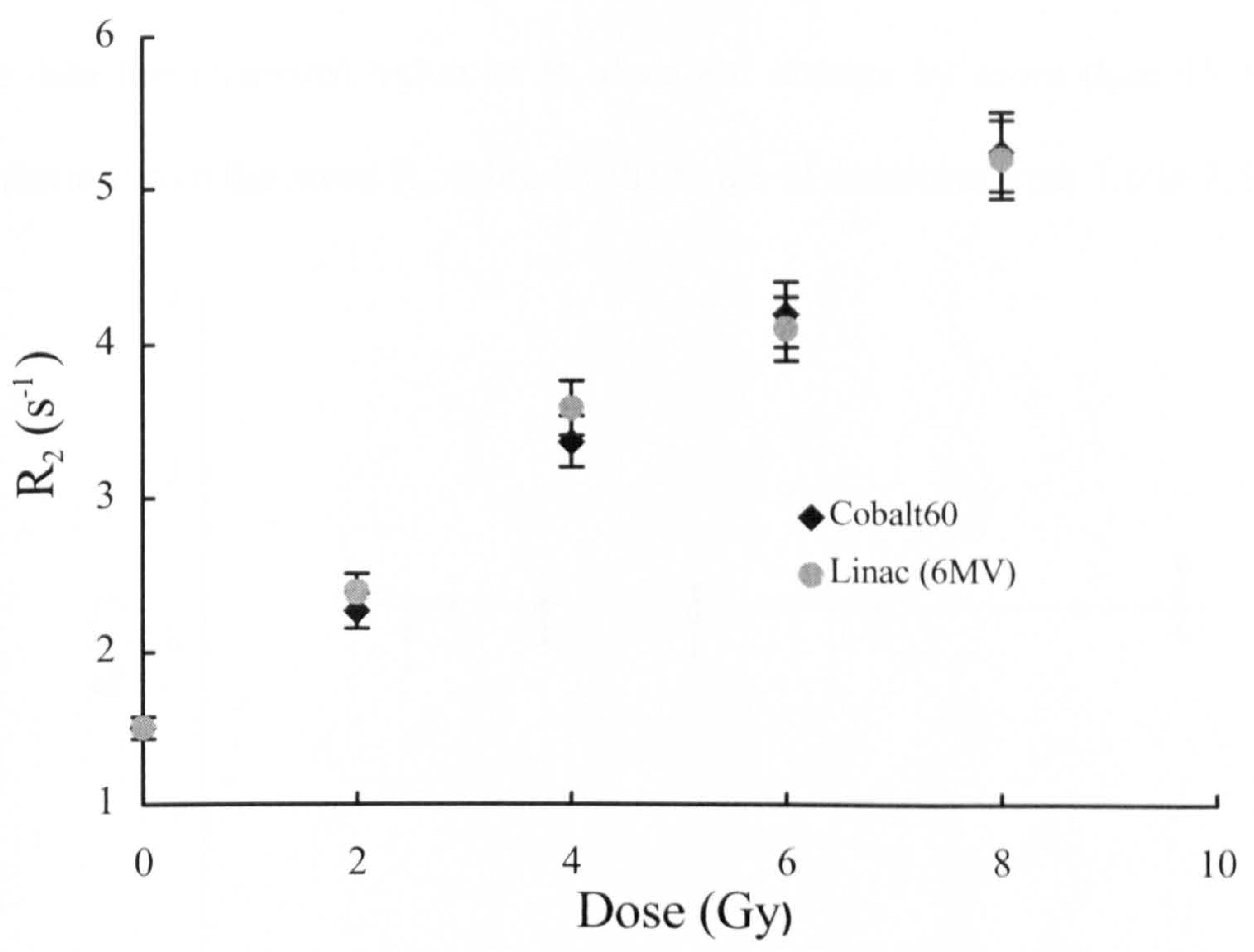

Figure 3.14. The effect of the continuous and pulsed radiation on the gel response

\subsubsection{Dose rate dependence}

The dependence of the gel response on doserate was also investigated. $350 \mathrm{ml}$ of the gel was prepared and 7 vials were filled. Exposures were made using an $8 \mathrm{MV}$ linear accelerator. Dose rate was varied by varying SSD. The radiation beam was calibrated at $71,85,100,120,140$ and $155 \mathrm{~cm}$ from the source to give absorbed dose of 6 Gy for each distance setting. A vial of BANG gel was irradiated to equal dose at each SSD, but over different times immediately after each calibration. Each gel was irradiated at a different doserate in the range of 1.6 to $7.62 \mathrm{~Gy} \mathrm{~min}^{-1}$. 
Figure 3.15 illustrates the $\mathrm{R}_{2}$ measurement for an absorbed dose of $6 \mathrm{~Gy}$. From the data the measured value of $R_{2}$ does not change by more than $\pm 5 \%$ maximum deviation from the mean $R_{2}$ value for the range of doserates from 1.6 to $7.62 \mathrm{~Gy} \mathrm{~min}^{-1}$.

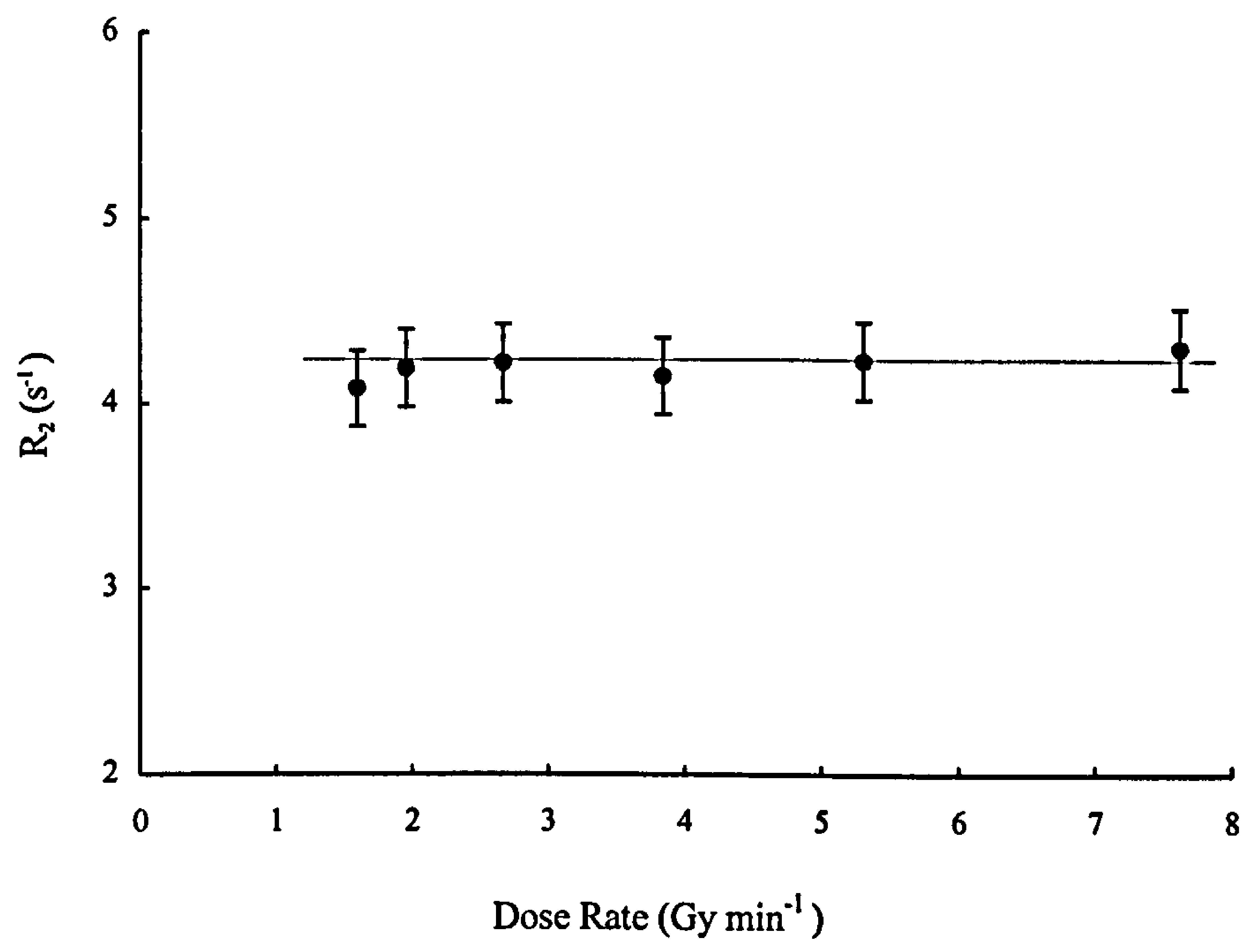

Figure 3.15. Variation of the gel response with doserate

\subsubsection{Diffusion}

In order to confirm that there is no diffusion of the polymer inside the gel, an investigation was made to measure the variation width of an irradiated area with time. A gel was exposed to a dose of $5 \mathrm{~Gy}$, using a field size of $4 \times 4 \mathrm{~cm}$, was measured on several occasions over a period of one month. Figure 3.16 shows the results of the measurements of the width of the image. It can be seen that there is no difference in image width between one and 30 days after irradiation. The width of the image was defined as the distance between the $50 \%$ values of $\mathrm{T}_{2}$ in the central region. 


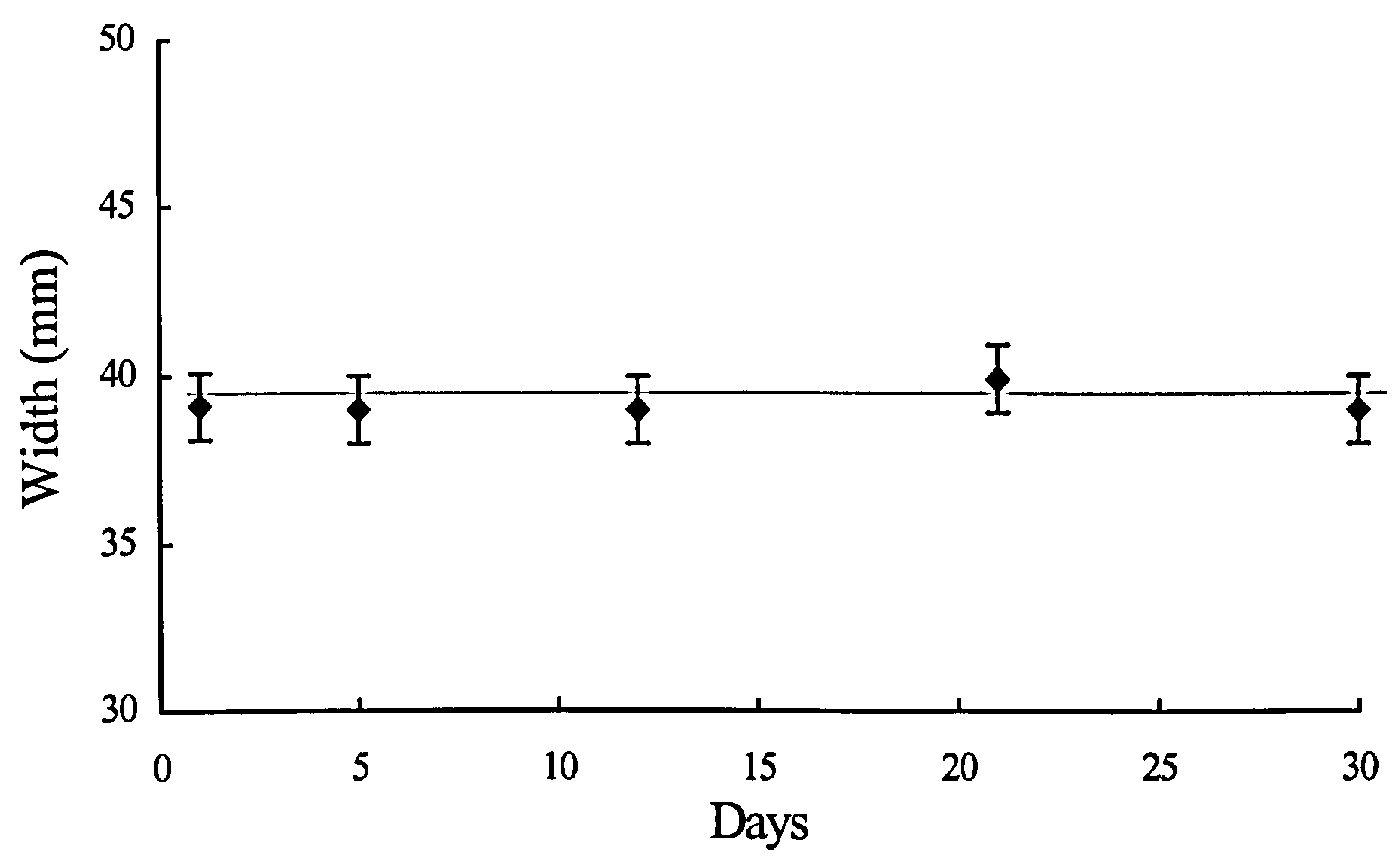

Figure 3.16. Diffusion of the polymer with time

\subsubsection{Temperature effect}

To confirm dependence of the gel response to temperature at time of MR imaging a set of gels was imaged at two different gel temperature. The temperature of one gel at the time of imaging was $13{ }^{\circ} \mathrm{C}$ and the other $23.5^{\circ} \mathrm{C}$. The results of the measurements are shown in figure 3.17. It is clear that the temperature at the time of image acquisition has a significant effect on results. The slope of the dose response curve decreases from $0.33 \pm 0.02$ at $13{ }^{\circ} \mathrm{C}$ to $0.23 \pm 0.02 \mathrm{~s}^{-1} \mathrm{~Gy}^{-1}$ at room temperature. The maximum transverse relaxation rate decreased from $4.75 \mathrm{~s}^{-1}$ at $13{ }^{\circ} \mathrm{C}$ to $3 \mathrm{~s}^{-1}$ at room temperature. 


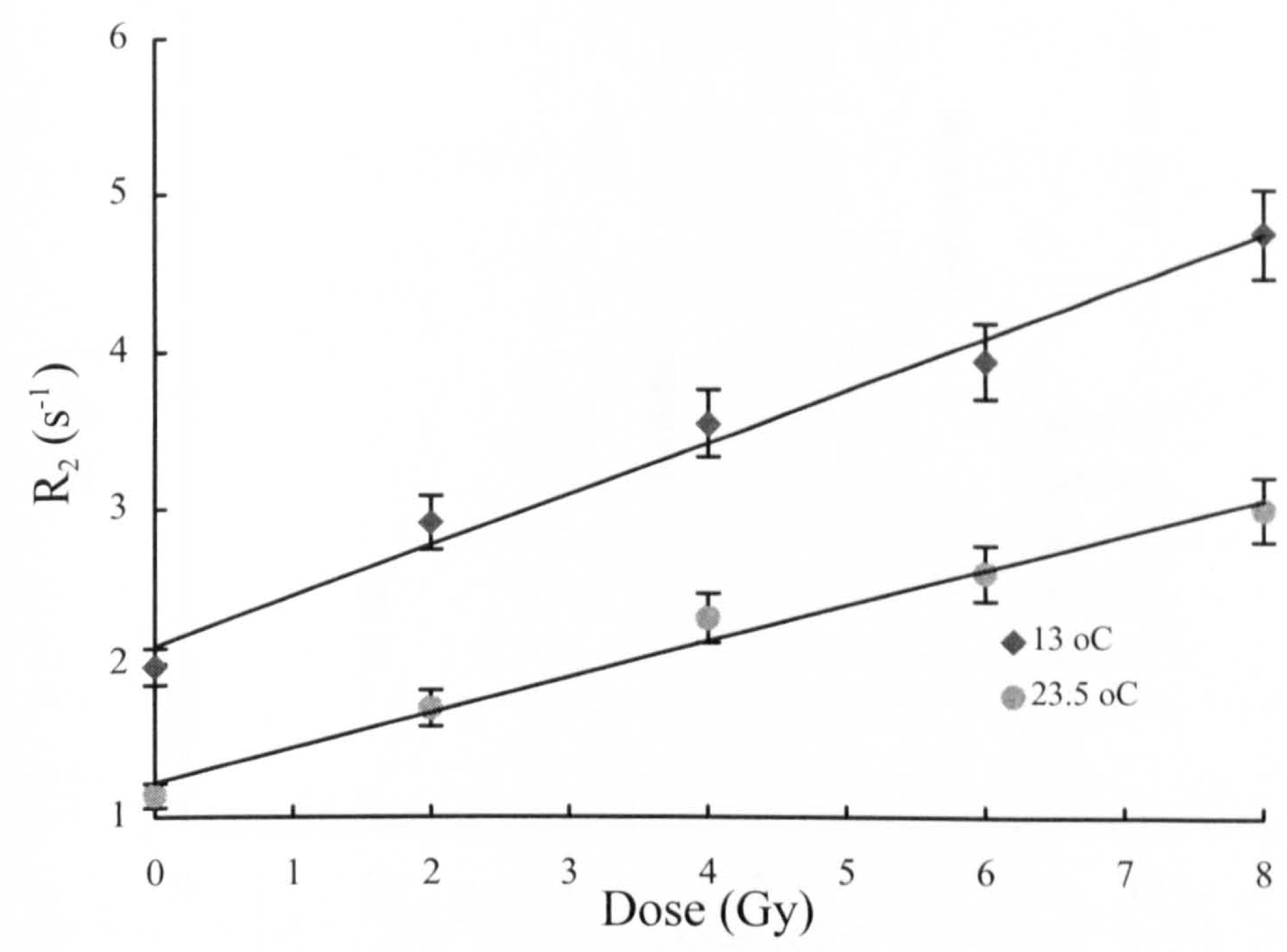

Figure 3.17. Temperature effect on $\mathrm{R}_{2}$ measurement

\subsubsection{Effect of the time of imaging after irradiation}

The variation of $R_{2}$ value with the time after irradiation was also investigated. The $R_{2}$ values of a series of gels was measured for a period of one month, during which five measurements were made. Figure 3.18 shows the measured $R_{2}$ for doses of 0 to 12 Gy for five different occasions during a month. It is clear that the response of the gel remains unchanged from the second measurement (5 days after radiation) for the higher doses. To determine when the $R_{2}$ measurements stabilise during the first five days, another set of the gels was irradiated and the response was measured over five consecutive days. Figure 3.19 shows the results of $\mathrm{R}_{2}$ measurement in the dose intervals of 0 to $12 \mathrm{~Gy}$. From the results it was found that the response of the BANG gel remains constant after two days. 


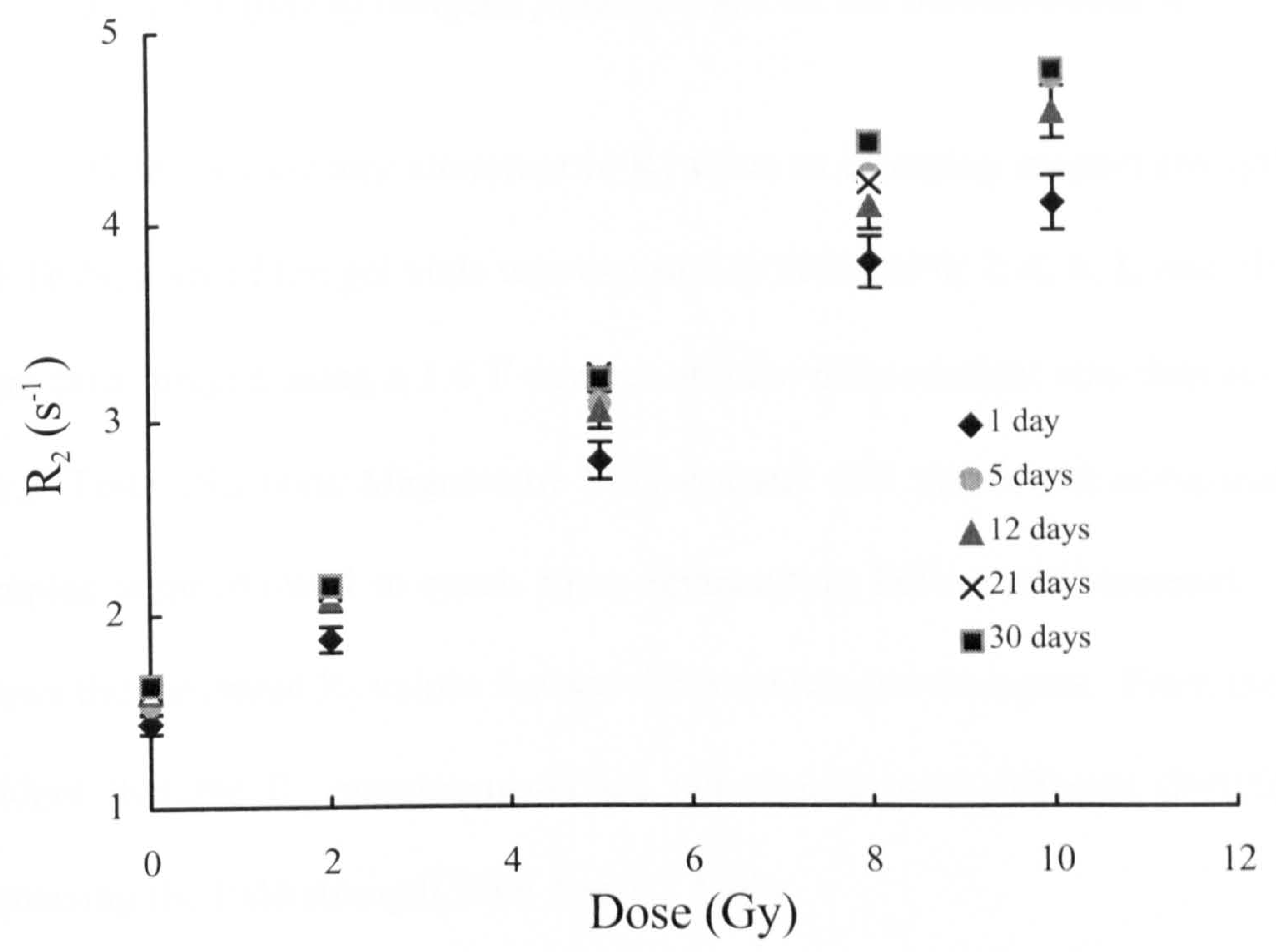

Figure 3.18. Variation of the gel response with time

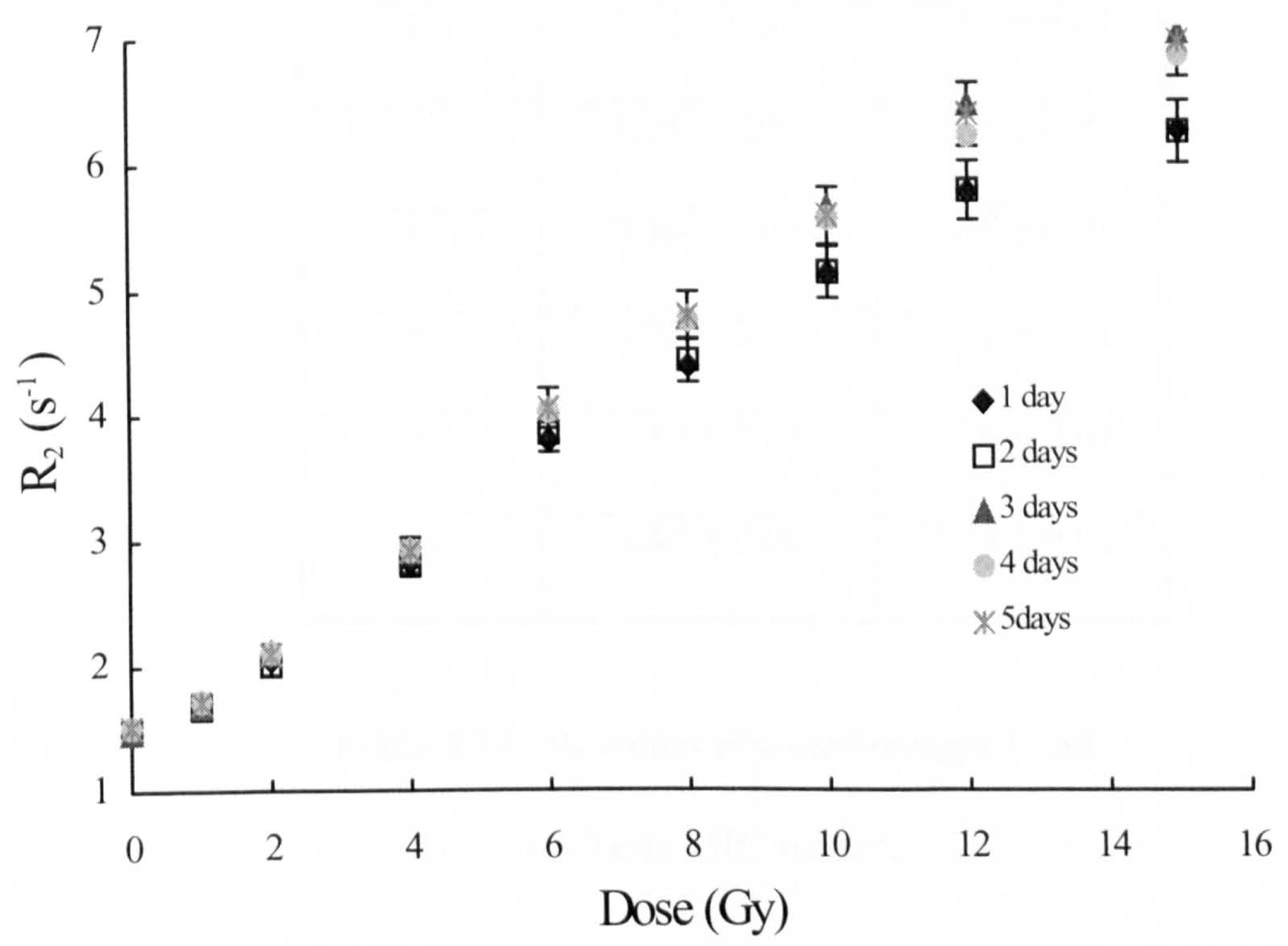

Figure 3.19. Stability of the gel response with time 


\subsubsection{Effect of imaging field strength on the measurement $\boldsymbol{R}_{2}$}

To determine any alteration in $R_{2}$ value in changing magnet strength from 1 to 1.5 Tesla, a set of the gel vials was exposed to doses of $0,2,4,6,8$, and $10 \mathrm{~Gy}$. These were then imaged using a $1.0 \mathrm{~T}$ scanner and the measurement was then repeated using a 1.5 Tesla (Siemens Magnetom) MRI scanner and the results compared. The gel samples were allowed to reach room temperature before measurement. Table 3.13 shows the measured $R_{2}$ values for two different magnet strengths. From the results it is evident that the $R_{2}$ measurements are not significantly different $(P<0.05$ t-test) by increasing the field strength from 1 to 1.5 Tesla.

\begin{tabular}{|c|c|c|}
\hline $\begin{array}{c}\text { Dose } \\
(G y)\end{array}$ & $\begin{array}{c}\mathrm{R}_{2} \text { values }\left(\mathrm{s}^{-1}\right) 1 \\
\text { Tesla }\end{array}$ & $\begin{array}{c}\mathrm{R}_{2} \text { values }\left(\mathrm{s}^{-1}\right) \\
1.5 \text { Tesla }\end{array}$ \\
\hline \hline 0 & $1.42 \pm 0.01$ & $1.41 \pm 0.01$ \\
\hline 2 & $1.95 \pm 0.01$ & $2.01 \pm 0.01$ \\
\hline 4 & $2.46 \pm 0.01$ & $2.45 \pm 0.01$ \\
\hline 6 & $2.77 \pm 0.01$ & $2.82 \pm 0.01$ \\
\hline 8 & $3.19 \pm 0.01$ & $3.26 \pm 0.01$ \\
\hline 10 & $3.42 \pm 0.01$ & $3.44 \pm 0.01$ \\
\hline
\end{tabular}

Table 3.13. $R_{2}$ values obtained using $a 1$ and

1.5 Tesla MRI scanner 


\subsubsection{Measurement of absolute dose}

Early results evident from the application of polymer gel dosimeters in different external beam therapy configurations revealed differences between the absorbed dose measured in the gel and that prescribed. Table 3.14 shows differences of up to $20 \%$ between measured and given dose. To determine the possible source of this difference several factors were investigated. These were sensitivity to light, effect of multiple exposure and method of calibration.

\begin{tabular}{|c|c|c|c||}
\hline \hline Applications & $\begin{array}{c}\text { Prescribed Dose } \\
(\mathrm{Gy})\end{array}$ & $\begin{array}{c}\text { Measured Dose } \\
(\mathrm{Gy})\end{array}$ & $\begin{array}{c}\text { Differences } \\
(\%)\end{array}$ \\
\hline Inhomogenity & 8 & $9.3 \pm 0.5$ & 14 \\
\hline 3 Field Glass & 10 & $12 \pm 0.6$ & 17 \\
\hline 3 Field Perspex & 10 & $11.25 \pm 0.6$ & 11 \\
\hline 4 Field Perspex & 10 & $11.85 \pm 0.7$ & 16 \\
\hline Conformal & 6 & $7.5 \pm 0.4$ & 20 \\
\hline Blood irradiation & $8 \pm 0.2$ & $8.3 \pm 0.3$ & 4 \\
\hline \hline
\end{tabular}

Table 3.14. Absolute dose measurements in the irradiated gels

It has been reported (Maryanski et al 1994) that polymer gels are sensitive to light and photo-polymerization could occur upon exposure to light sources. To assess the sensitivity of the gel, two different gels were exposed to the field light from the accelerator. Firstly an area of $4 \mathrm{~cm}^{2}$ from 3 litre gel phantom was exposed for $10 \mathrm{~min}$ 
to the Linac field defining light and compared with an unexposed area. The second investigation involved five vials of the gel. These were filled and irradiated to an absorbed dose of $6 \mathrm{~Gy}$. Prior to irradiation one vial was kept light tight but vials two, three, four and five were left 1, 3, 5, and 7 min under the light respectively. Table 3.15 shows the results of measurement for both phantom and vials. From the results it is clear that the gel is not affected by the light beam, at least in the range of these measurements.

\begin{tabular}{||c|c|c|c|c|c|c||}
\hline \multicolumn{6}{|c||}{$\mathrm{R}_{2}$ values (s-1) for corresponding length of light exposures (min) } \\
\hline & 0 & 1 & 3 & 5 & 7 & 10 \\
\hline Vials & $3.70 \pm 0.02$ & $3.85 \pm 0.03$ & $3.77 \pm 0.03$ & $3.70 \pm 0.02$ & $3.66 \pm 0.02$ & - \\
\hline Phantom & $1.60 \pm 0.03$ & - & - & - & - & $1.59 \pm 0.02$ \\
\hline \hline
\end{tabular}

Table 3.15. Sensitivity of the gel to radiation field light

It was possible that the gel response may be altered by the number of consecutive irradiations i.e. successively sensitised. Therefore a gel phantom of 3 litre capacity was irradiated with two radiation fields of $3 \times 3 \mathrm{~cm}$ with a $3 \mathrm{~cm}$ gap between them. One of the fields was given an absorbed dose of $6 \mathrm{~Gy}$ to the isocentre but for the other the $6 \mathrm{~Gy}$ was given in 4 fractions of $1.5 \mathrm{~Gy}$ with five minutes delay between them. This measurement was also repeated using 3 glass vials given $6 \mathrm{~Gy}$ in one, two, and four fractions.

The results for both measurements are shown in table 3.16. No significant difference was found in gel response with multiple exposures with $\mathrm{P}<0.05$ using t-test.

For each measurement using the gels, glass vials were used to produce a calibration curve. To verify any differences using a single large container comparison was made between irradiating small glass vials and a phantom to several known doses. 
In both cases the gel was from the same batch of manufacturing and imaged at the same temperature.

Figure 3.20 shows the calibration curves that were obtained using the usual method of calibration compared with phantom irradiation. From the results it is clear that phantom calibration gives a different slope $0.33 \pm 0.003 \mathrm{~s}^{-1} \mathrm{~Gy}^{-1}$ than that of the vials $0.24 \pm 0.01 \mathrm{~s}^{-1} \mathrm{~Gy}^{-1}$.

\begin{tabular}{||c|c|c|c||}
\hline \hline \multicolumn{4}{||c||}{$\mathrm{R}_{2}$ values $\left(\mathrm{s}^{-1}\right)$ of $6 \mathrm{~Gy}$ for multiple exposure } \\
\hline & 1 & 2 & 4 \\
\hline Vials & $3.66 \pm 0.02$ & $3.74 \pm 0.03$ & $3.71 \pm 0.03$ \\
\hline Phantom & $3.37 \pm 0.08$ & - & $3.43 \pm 0.09$ \\
\hline
\end{tabular}

Table 3.16. Variation of gel response with multiple exposure

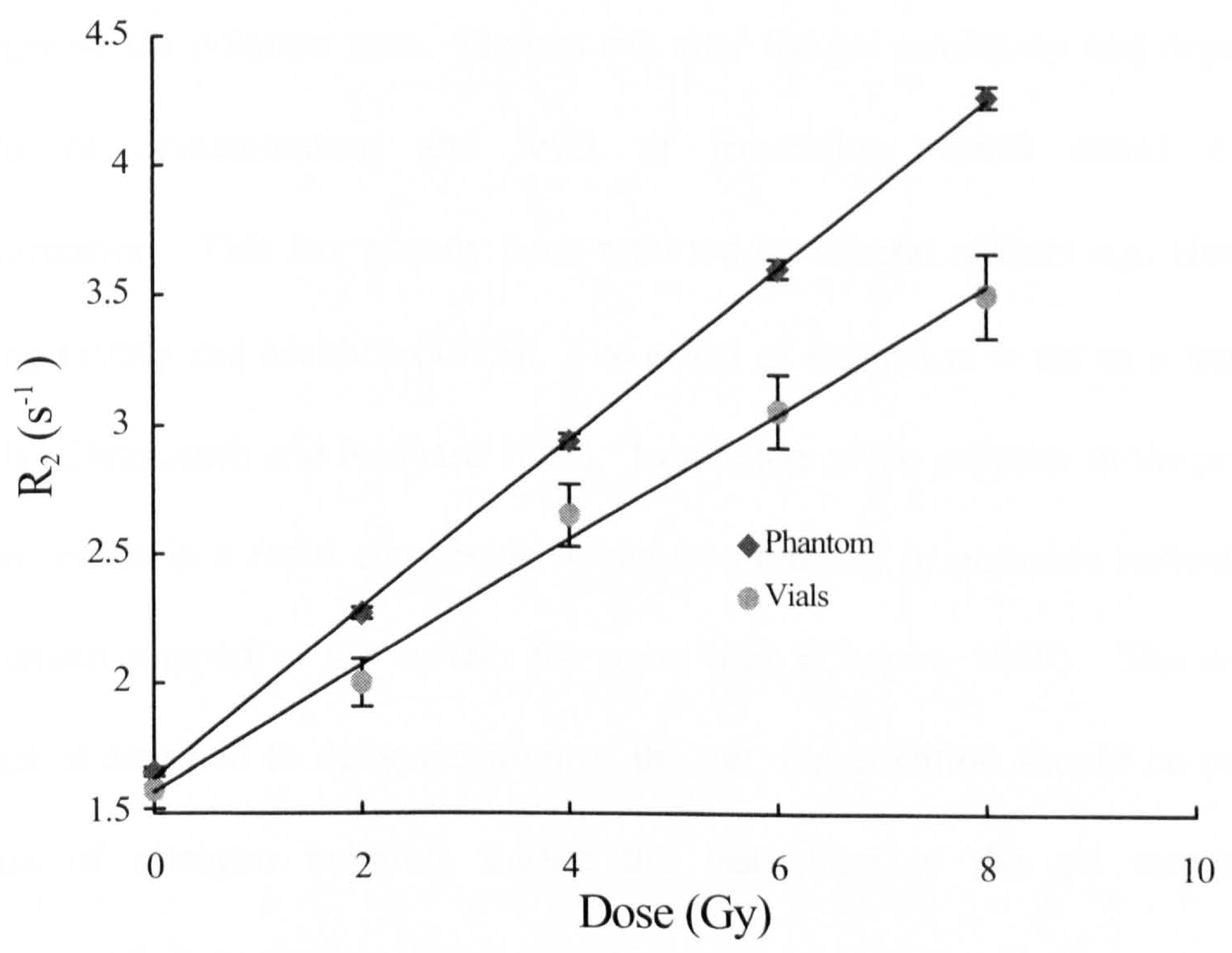

Figure 3.20. Comparison of the different method of calibration 


\subsection{Discussion}

Polyacrylamide gels have recently caused much interest in both radiation dosimetry, and NMR studies. They are a very convenient material and have the potential to be used in any complex configuration. They are normally soft tissue equivalent but by altering their components Wielopolski and his colleagues (1985) made polyacrylamide bone phantom to simulate wet cortical bone. In addition Kennan et al (1996) used polyacrylamide gels to study MR relaxation mechanisms of tissue as their NMR properties are similar to biological tissue.

Radiation dosimetry takes advantage of polymerization and cross-linking of the polymer monomer upon irradiation. In order to achieve polymerization it is necessary to remove any oxygen from the gel. Nitrogen or nitrous oxide gases have been used to deoxygenate the polymer gels. Oxygen can alter the gel sensitivity and depending on amount of contamination and level of irradiation, could retard or inhibit polymerization. This has already been reported by several authors e.g. Hoecker and Watking (1958) and Makhlis (1975). The effect of oxygen is to act as a trap for free radicals (Chrambach and Rodbard 1983). Irradiation of the polymer in the presence of oxygen results in a rapid conversion of the free radicals to peroxide radicals. These may remain trapped in the system for some time (Chapiro 1967). The role of the nitrogen is confined to deoxygenation of the gel, but attention should be paid to the duration of nitrogen bubbling during the last stage of the gel manufacturing. Spontaneous polymerization that occurred in some gels is more likely due to prolonged nitrogen bubbling of the polymer solution before gelation. It is also possible that impurities in chemicals can be a cause of autopolymerization (Bio-Rad Bulletin 1987). 
As a high purity monomer was used it would appear that gelatin is a more likely source of contamination (Maryanski et al 1994).

The gel was found to be thoroughly homogeneous after gelation, as measured relaxation rates in different points inside the phantom have shown no differences. The result of the investigation using a large volume of the gel has demonstrated that uniform $\mathrm{R}_{2}$ distribution could be achieved using a Hahn spin-echo pulse sequence. This is in agreement with the results of investigations carried out by Maryanski and Gore (1992) in $\mathrm{MnCl}_{2}$ solution using both Hahn spin-echo and multi-echo Carr-Purcell pulse sequences (2.5.2).

The BANG gel was observed to become visibly cloudy at an absorbed dose of about $1 \mathrm{~Gy}$. In the BANANA gel the minimum dose required for the observation of polymerization has been reported to be 5 Gy (Maryanski et al 1993, Djennaui 1994). The reason for this is that gelatin gels are more transparent than agarose gels.

The sensitivity of the gel, was found to depend on the concentration of the total monomer. For example the sensitivity of the gel was increased by $79 \%$ in changing from $6 \%$ to $9 \%$ of monomer, using equal amounts of bis and acrylamide. Therefore to make a more sensitive gel requires higher concentration of total monomer. This is in agreement with work reported by Baldock et al (1996c). They found a $100 \%$ increase in the slope of the response curve by doubling the concentration of the total monomer. A similar effect has been reported for chemical initiators, where changing from 5 to 30 $\%$ of the monomer results in a reduction of 20 to $50 \%$ of initiator concentration (BioRad Bulletin 1987). Since a high concentrations of the total monomer is required to produce a sensitive gel, a concentration of $8 \%$ of the total monomers was chosen for the final recipe. The limiting factor in increasing the percentage of total monomer 
however is the longer preparation time due to the low solubility of the bis powder and also formation of the crystals. It has been reported that low temperature water is not an ideal solvent for bis (Righetti et al 1983), therefore to help dissolve the bis powder the gel solution should keep heated during magnetic stirring. It was observed on top of the gel that was prepared using $9 \%$ of total monomers crystalline salt was formed one week after preparation. A similar problem was observed with BANANA gel using $8 \%$ of total monomer (Maryanski et al 1993), however the preparation carried out in this study using $8 \%$ of total monomer does not result in the formation of crystalline salt.

The results of these investigations demonstrated the reproducibility of the gel response to within $\pm 4 \%$ for gels manufactured from the same batches of chemicals. However using chemicals from different batches results in a large difference in dose response. The relationship between absorbed dose and relaxation rate was found to be linear up to about 10-12 Gy. The dose response of BANG gel was initially reported to be linear up to $8 \mathrm{~Gy}$ by Maryanski et al (1994) and then to $12 \mathrm{~Gy}$ (Maryanski et al 1996a).

Increasing the fraction of acrylamide from 3 to $4 \%$ did not offer any advantages. In contrast the same amount of increase in bis appeared to increase linearity of the gel response up to $15 \mathrm{~Gy}$ but with no improvement in the sensitivity of dose response. This was unexpected because the total amount of monomer has been changed from 6 to $7 \%$. The reason could be the increased contribution of bis to the total monomer. This agrees with the recent work reported by Maryanski et al (1997a).

The role of gelatin in the polymerization process is not well understood (Maryanski et al 1994) and it has been postulated that its role is only to control the spread of polymerization. It was found however that increasing the concentration of 
gelatin will reduce the sensitivity of the gel response. Studies of the effect of gelatin concentration on dose response carried out by Audet et al (1995) agree with the results of this investigation.

The major disadvantage in using Fricke gel dosimetry is the diffusion of the ferric ions (Schulz et al 1990). Therefore the lack of polymer diffusion throughout the gel is a considerable advantage over the Fricke gel. The study of the image width confirmed this, since no changes were found. This makes polymer gel a very useful means of measuring dose distributions. They can be measured at any convenient time after irradiation.

The increase in values of $R_{2}$ was observed over the first two days following irradiation after which they become constant. The change was much more pronounced at higher doses. This may be explained by the release of trapped radicals and further reactions with the monomer (Makhlis 1975). The fate of the trapped radicals depends on the physical state of the polymer. Their reactivity is significantly reduced if they are embedded in a viscous medium and this could possibly be the reason for large changes at higher doses. In some cases radicals can remain trapped for extremely long periods of the order of several days or even months (Chapiro 1962).

The slope of the dose response varies with the temperature of the gel during MR measurements, therefore special attention must be paid to gel temperature during different measurements to avoid mis-interpretation of the results. The amount of decrease in slope was found to be $0.009 \pm 0.0005 \mathrm{~s}^{-1} \mathrm{~Gy}^{-1}{ }^{\circ} \mathrm{C}^{-1}$. The effect of temperature on slope measured here confirmed the work which was reported by Maryanski et al (1995 and 1997). They found a reduction of about $0.01 \mathrm{~s}^{-1} \mathrm{~Gy}^{-1}$ in 
slope per ${ }^{\circ} \mathrm{C}$ increase. In addition they reported that temperature variation at the time of irradiation had no effect on dose response.

The study of dose response of BANG polymer gel dosimeter as a function of doserate and beam energy demonstrated that the gel dose response is independent of energy over the range 0.3 to $8 \mathrm{MV}$ and doserate from 1.6 to $7.62 \mathrm{~Gy} \mathrm{~min}^{-1}$. This confirms the claim that was made by Maryanski et al (1996a). They reported that the response of the gel is independent of doserate in the range of $20-400 \mathrm{cGy} / \mathrm{min}$ and beam energies up to $15 \mathrm{MeV}$, but produced no direct experimental evidence.

It has been reported (Maryanski et al 1993) that the slope of the dose response increases by $27 \%$ by changing from a $0.5 \mathrm{~T}$ scanner to $1.5 \mathrm{~T}$ and $139 \%$ from a 1.5 to a $2 \mathrm{~T}$ scanner. In the present study it was found that using a $1.5 \mathrm{~T}$ MR scanner instead of a $1 \mathrm{~T}$ scanner has no effect on sensitivity of the dose response.

A problem was found to arise in the absolute measurements of absorbed dose. The explanation for this may lie in the method of absorbed dose calibration for the gel (Farajollahi et al 1997). Oxygen is the main source of variation in response in polymer dosimetry. It was inferred that the amount of oxygen can alter the gel sensitivity and depending on the magnitude of the absorbed dose can prevent polymerization. For example it was observed that a gel did not respond to low doses up to $5 \mathrm{~Gy}$, polymerized at doses higher than $5 \mathrm{~Gy}$. This fact has also been highlighted by Chapiro (1962). Therefore the role of oxygen in gel manufacturing and the calibration method is the key factor in accurate measurements of absolute dose. It is believed that the differences in oxygen concentration in the calibration and irradiated gels, even using the same batch of chemical, will result in different sensitivity and consequently difference in absolute dose. Ideally the same phantom should be used for both 
calibration and measurement or a method of measuring oxygen concentration in the gel should be developed. Alternatively a means of excluding all oxygen from the prepared gel samples should be introduced. More sophisticated oxygen monitoring, for example in the glove box could also be used. However if relative dose measurements are required the effects of calibration and gel temperature on MRI measurements are not important. In this case the main requirement would be the measurement of background. 


\section{Chapter 4. Application of BANG gel in brachytherapy}

\subsection{Introduction}

The measurement of absorbed dose distributions in brachytherapy is often difficult due to steep dose gradients (Godden 1988). In addition the arrangement of sources in clinically relevant applications can also be quite complex. Therefore any dosimetric method used in brachytherapy must be capable of measuring dose distributions with high resolution in three dimensions, with a response that is independent of energy and doserate.

Conventional dosimeters such as ionization chambers, semiconductor detectors, thermoluminescent dosimeters (TLDs) and films have their own limitations. Films have good spatial resolution but are not tissue equivalent (Thomas et al 1992), are difficult to manipulate in complex geometries and also their sensitivity has been shown to vary with field size and depth in phantom (Burch et al 1997). TLDs are good integrating dosimeters, but the measurement of a true three dimensional dose distribution is laborious and time consuming as TLDs will only provide a matrix of point doses.

MRI Fricke gel dosimeter, introduced in 1984 by Gore et al, has provided a powerful dosimetric method that measures 3D dose distribution with high resolution in a tissue equivalent medium (Olsson et al, 1989). This method is based on the radiation-induced conversion of ferrous ion to ferric which can be measured using MRI, as has been discussed earlier. The unique properties of Fricke gel made it potentially an ideal dosimeter for brachytherapy. Its suitability in both high doserate (HDR) (Olsen \& Hellesnes 1994, Schreiner et al 1994) and low doserate (LDR) brachytherapy (Olsen \& Hellesnes 1994) has been investigated. However the major concern with the use of the Fricke system is the diffusion of ferric ions through the gel after irradiation which leads to blurring of the measured dose distribution with time, and a consequent loss of resolution (Schulz et al 1990, Olsson et al 1992). This effect 
limits its application particularly in LDR brachytherapy, therefore a dosimeter that does not suffer from this problem would be highly desirable.

The BANG polymer gel dosimeter that was discussed in chapter 3 does not suffer from the problems associated with the Fricke system and seems can be an excellent tool for measuring dose distribution in brachytherapy.

This chapter is aimed at investigating the applications of BANG gel in simple and complex LDR brachytherapy.

\subsection{Methods and Materials}

3 litres of BANG gel were prepared as described (3.6.1). Then eight small glass vials were filled with the gel for calibrating the dose response of the gel together with the phantom to be irradiated. The phantom was a cylindrical Perspex container, 150 $\mathrm{mm}$ in diameter and $190 \mathrm{~mm}$ in height with a central Perspex tube to accommodate a brachytherapy applicator (figure 4.1). The gel samples for calibration were irradiated to absorbed doses of 0 to 12 Gy using $6 \mathrm{MV}$ photons from a Philips SL $75 / 5$ linear accelerator.

\subsubsection{MRI Imaging and data analysis}

The MR images were obtained five days after irradiation using a Siemens Impact whole body scanner operating at 1 Tesla $(\mathrm{T})$. The gel phantom was placed in the centre of the head coil and a multi slice spin-echo pulse sequence was used. All the measurements were made using a repetition time (TR) of $6000 \mathrm{~ms}$ and echo times (TE) of 15 and $400 \mathrm{~ms}$ to obtain $\mathrm{T}_{2}$-weighted images. The field of view was $190 \mathrm{~mm}$ and the matrix size was $128 \times 256$ with one acquisition and slice thickness of $3 \mathrm{~mm}$. The time taken to image the gel using these parameters was about half an hour. The images were then transferred to a Sun Work Station. The transverse relaxation time $\left(\mathrm{T}_{2}\right)$ of the water protons in the irradiated gels were then determined from the calculated $T_{2}$ image. 
The dose distributions were then obtained from the calculated $T_{2}$ image using Analyze ${ }^{\mathrm{TM}}$ image processing software by applying the calibration data.

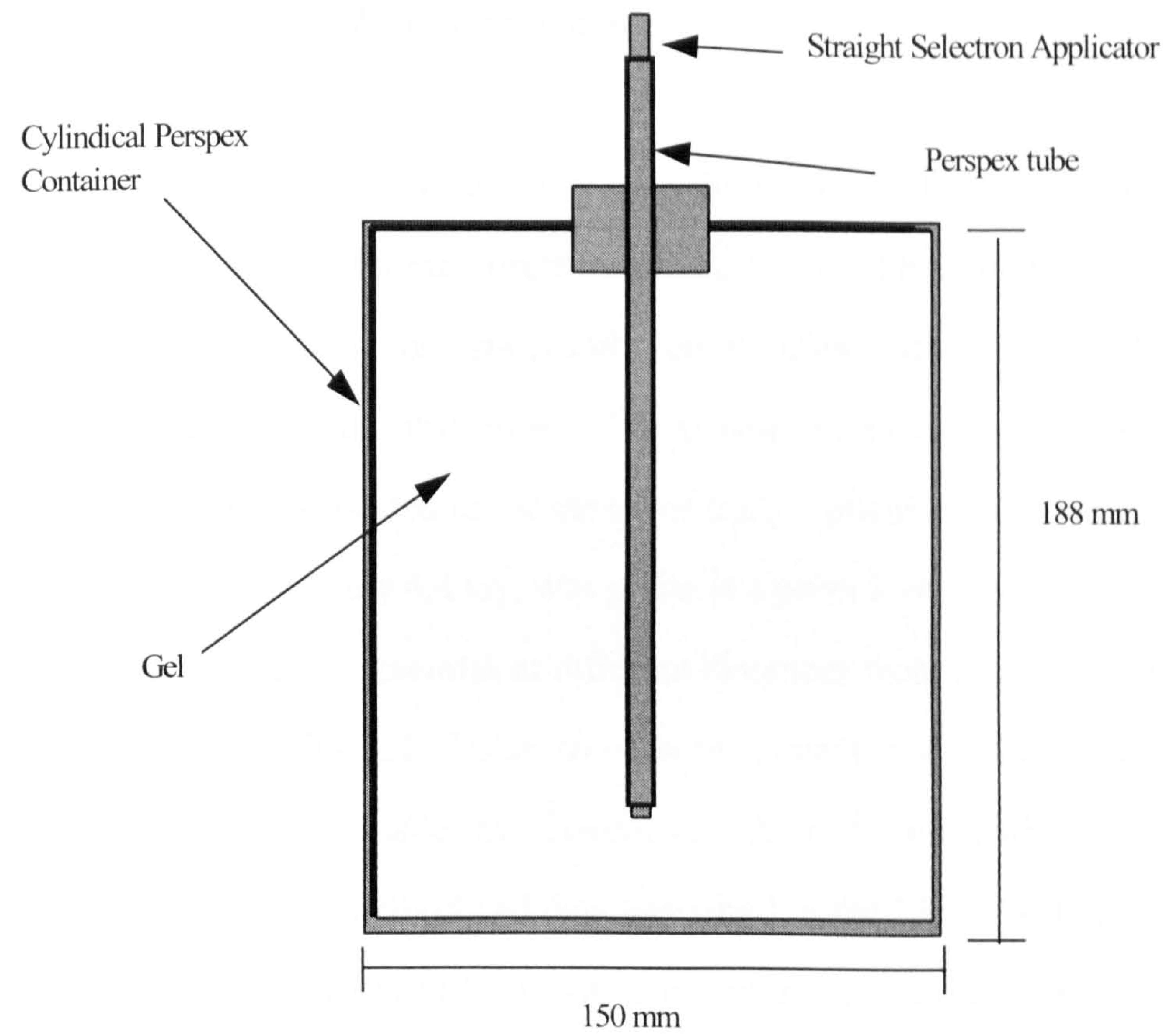

Figure 4.1. The phantom with applicator in central Perspex tube

\subsubsection{Overall uncertainty in the dose calculation}

In order to calculate dose from $R_{2}$ the equation, $R_{2}=R_{b g}+b . D$ was used to fit the calibration data, where $\mathrm{R}_{\mathrm{bg}}, \mathrm{b}$ and $\mathrm{D}$ are background $\mathrm{R}_{2}$, slope and absorbed dose respectively. The uncertainty was calculated for $\mathrm{R}_{2}$ minus background and then summed in quadrature with the error in the slope. The overall error was estimated to be a maximum of $5 \%$ up to $10 \mathrm{~Gy}$. For higher doses the amount of the uncertainty increases as the uncertainty in slope increases because of saturation effect. For example the error in dose measurement up to 14 Gy was estimated to be $10 \%$. For 
relative measurements the equation used $D_{1} / D_{\text {ref }}=R_{1}-R_{b g} / R_{\text {ref }}-R_{b g}$ where the subscript "ref" indicates the reference point.

\subsubsection{Simple LDR brachytherapy}

In collaborative experiments with the Medical Physics Department, Walsgrave Hospital, Coventry two measurements were made. The irradiations were carried out using a Selectron low doserate (LDR) remote afterloading system (Nucletron) with a stainless steel straight applicator. The system employs Cs-137 radioactive sources. The applicator was located in the centre of the gel phantom and using 36 active sources a dose of approximately $4.8 \mathrm{~Gy}$, was given at a point $2 \mathrm{~cm}$ from the centre of the source train. The measured doserates at different distances from the centre of the source were then compared with LiF TLDs from both Coventry and Leicester together with a calculation of dose made by Coventry. Prior to irradiation the doserates were calculated using the method and data described in the BIR/IPSM recommendations for brachytherapy dosimetry (1992). The applicator was also removed before MR imaging to avoid the disturbance of the magnet by the metal.

The TLDs were placed at $5 \mathrm{~mm}$ intervals in a cylindrical Perspex holder and irradiated in a water phantom. Each TLD was located in an individual holder and separated by spacers to produce a stack. Two stacks, one from each centre, could be irradiated simultaneously. This jig (figure 4.2) was designed by staff at the Walsgrave Hospital (Ratcliffe 1997).

For calibration the gel vials were irradiated in a Perspex phantom and also the radiation beam was calibrated using the same process as described earlier (3.7.4). The imaging condition for the vials was exactly the same as for the gel phantom. 


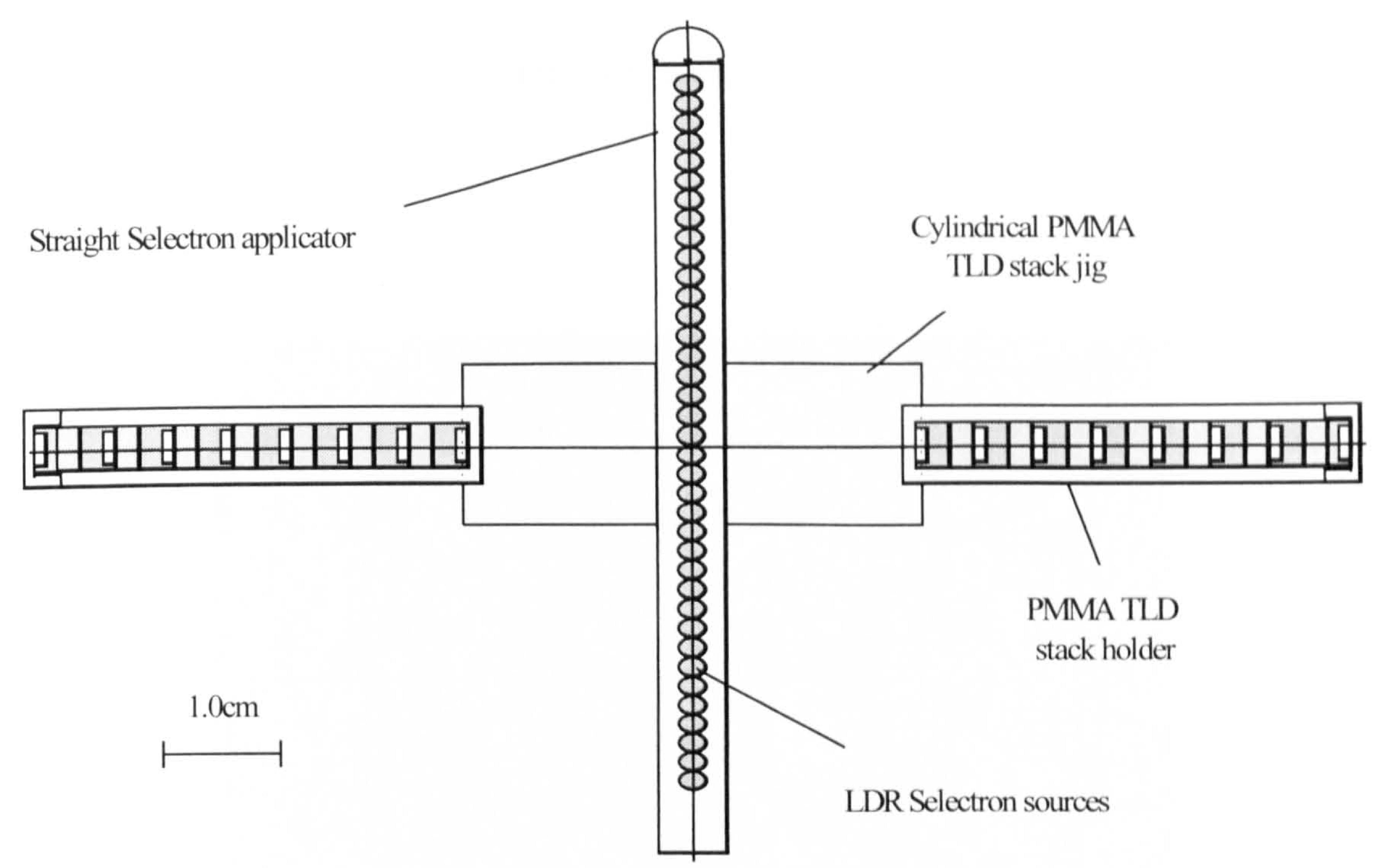

Figure 4.2. The jig used for irradiation of the TLDs.

The jig consists of two stacks supported by the Perspex tubes.

\subsubsection{Complex LDR brachytherapy}

In order to investigate the performance of the gel in a more complex geometry a realistic clinical treatment condition was simulated. Two specially designed phantoms were made and filled with the gel so that a gynaecological applicator could be sandwiched between them (Figure 4.3). The applicator was connected to the Selectron LDR and the gel was irradiated using Cs-137 sources. The applicator set consists of a $45^{\circ}$ inter-uterine tube of $6 \mathrm{~cm}$ length and two ovoids of $2.5 \mathrm{~cm}$ diameter. The source activity was $15.48 \mathrm{GBq}$, with total irradiation time of $240 \mathrm{~min}$ estimated to give approximately 6 Gy to the Manchester A point.

In order to compare the dose distribution from the gel with the planning computer the applicator position in the gel was determined from two orthogonal 
radiographs. The radiographs were then used to produce a plan using the Helax-TMS planning system.

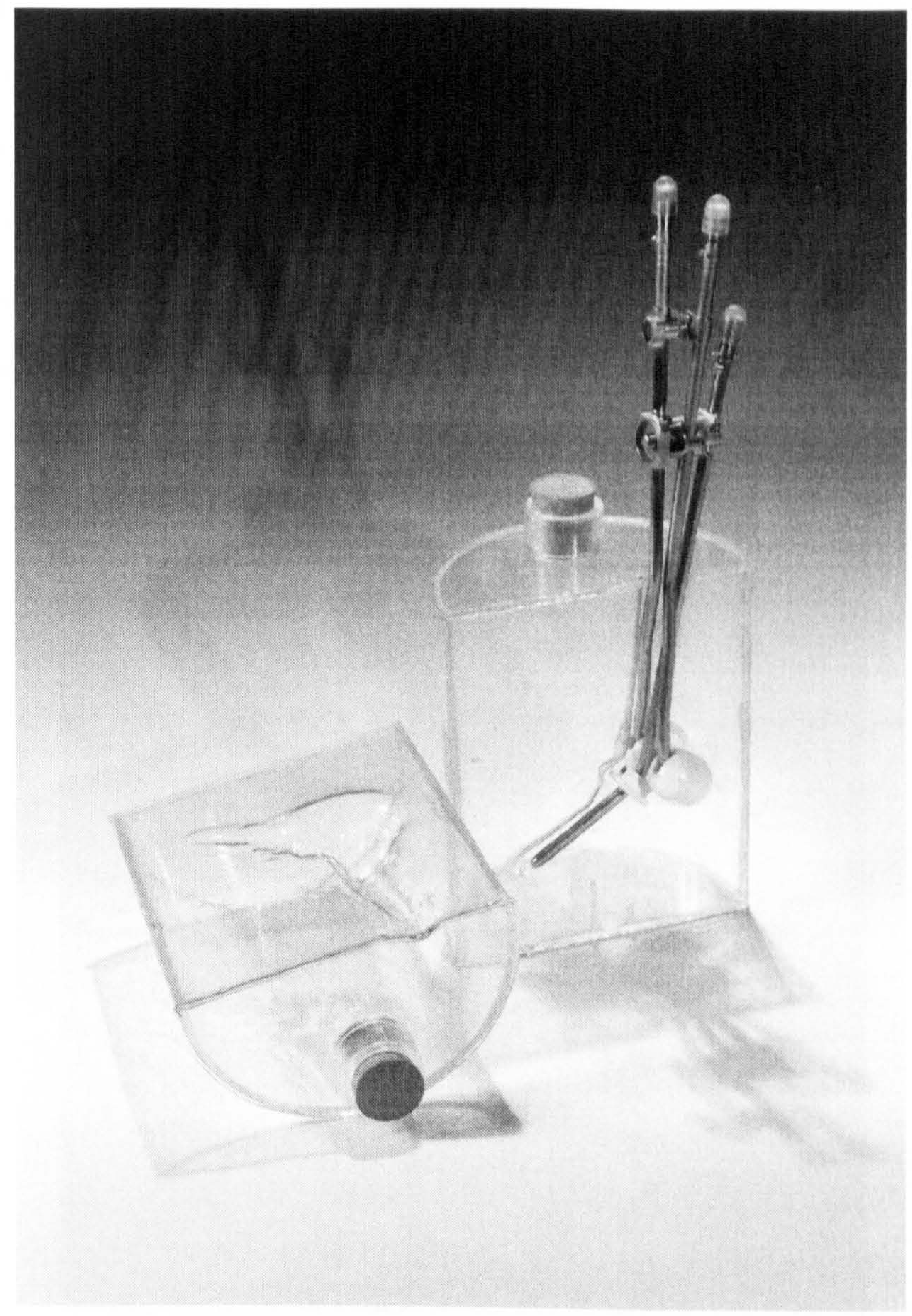

Figure 4.3. The experimental set up for complex brachytherapy 


\subsection{Results and discussion}

\subsubsection{Simple LDR brachytherapy}

A typical $T_{2}$ weighted image of the vials in the polystyrene holder and the calibration curve are shown in figure 4.4 and 4.5 respectively. The relaxation rate increases linearly with the dose and saturates at doses higher than 12 Gy.

Figure 4.6 shows the typical dose distributions in the gel produced in a sagittal plane. In order to demonstrate the three-dimensional nature of the gel a coronal view of the gel was also produced (Figure 4.7).

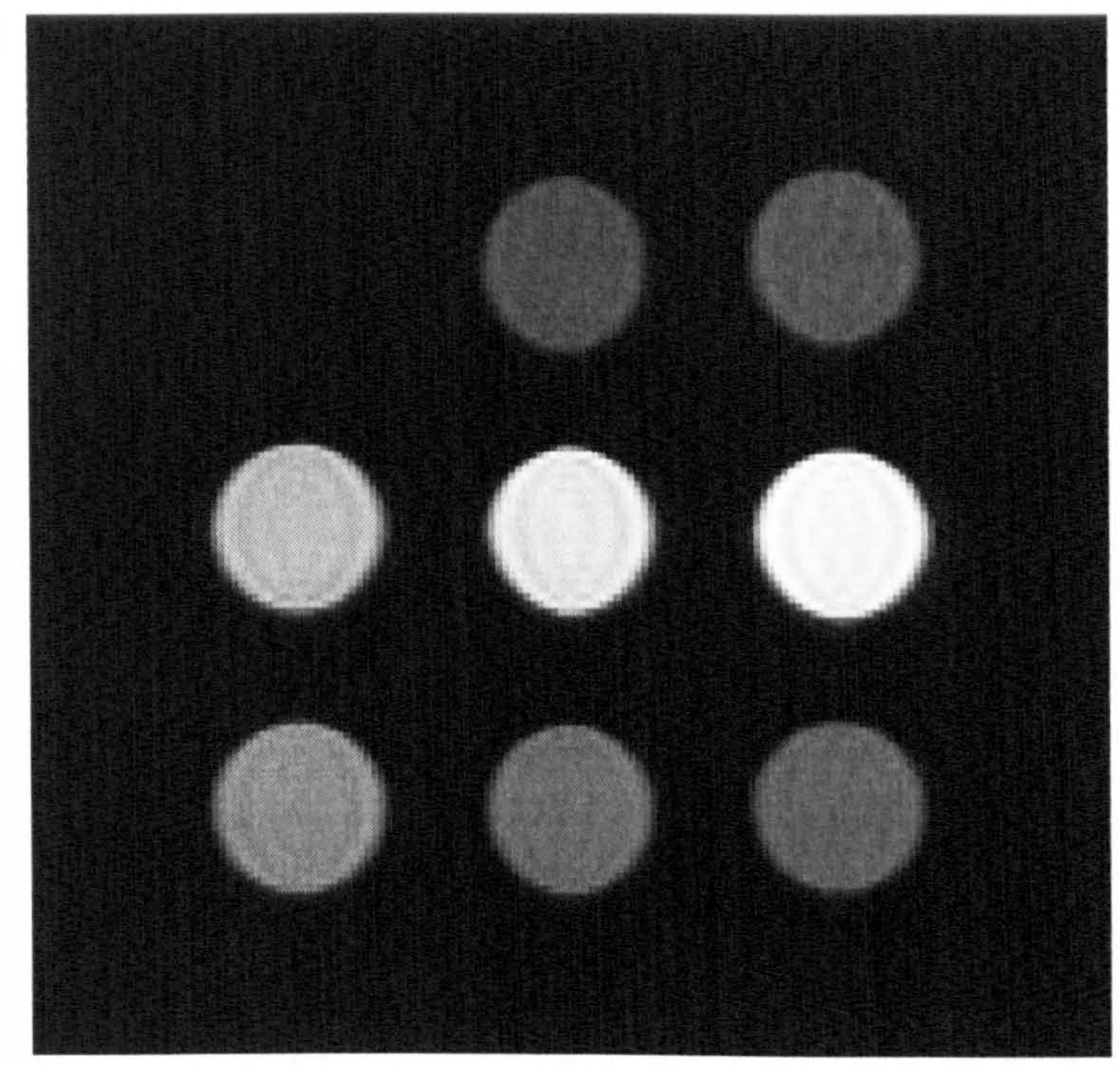

Figure 4.4. A typical $\mathrm{T}_{2}$ weighted image $(\mathrm{TR}=6000 \mathrm{~ms}$ and $\mathrm{TE}=400 \mathrm{~ms}$ ) of the calibration vials demonstrating the different signal intensity due to different radiation dose 


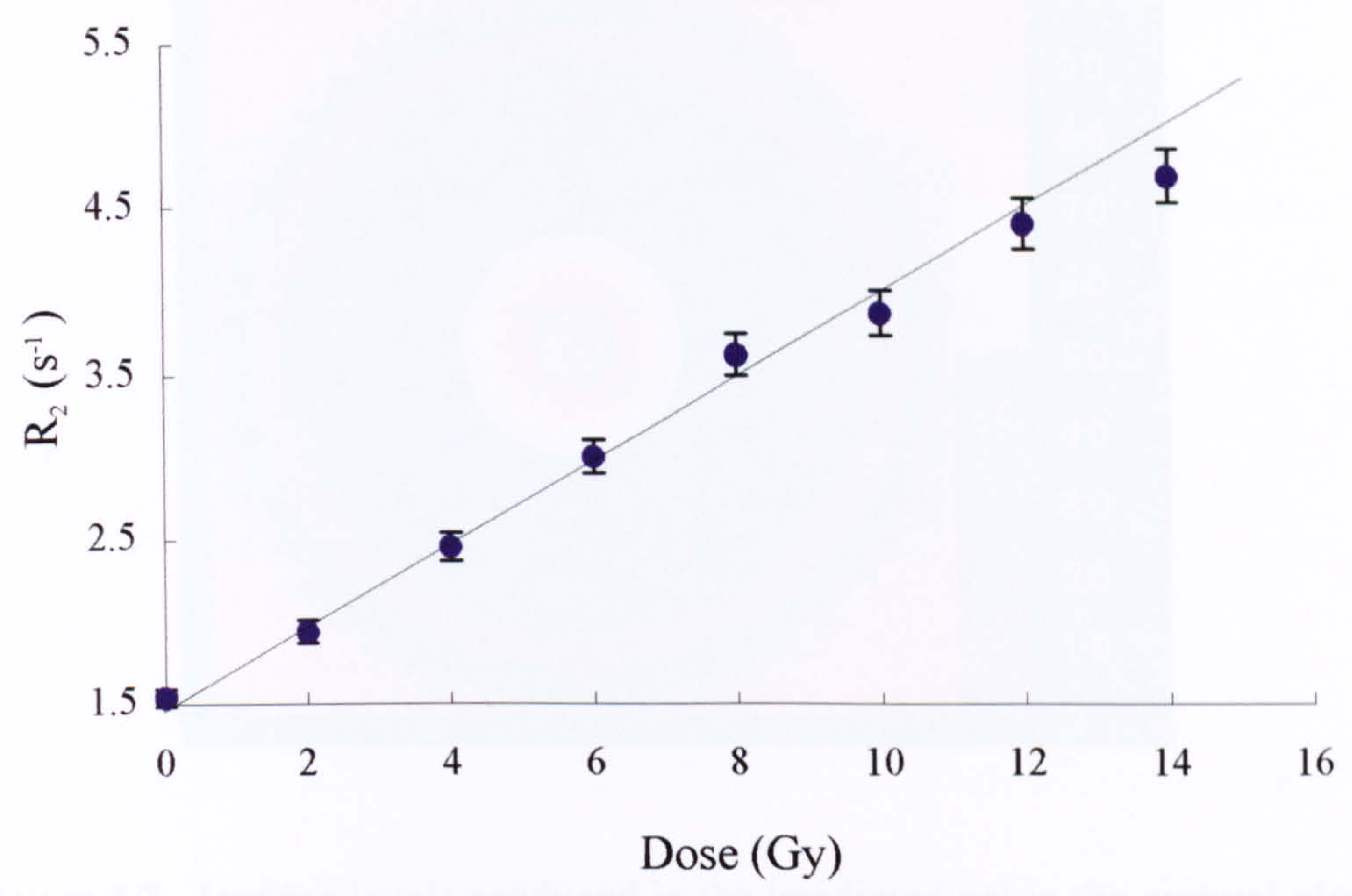

Figure 4.5. A typical calibration curve for the gel. The error of $3.5 \%$ is indicated for each data points. The linear data fit is

$$
\mathrm{R}_{2}=1.54+0.23 \mathrm{D}, \mathrm{r}=0.996
$$

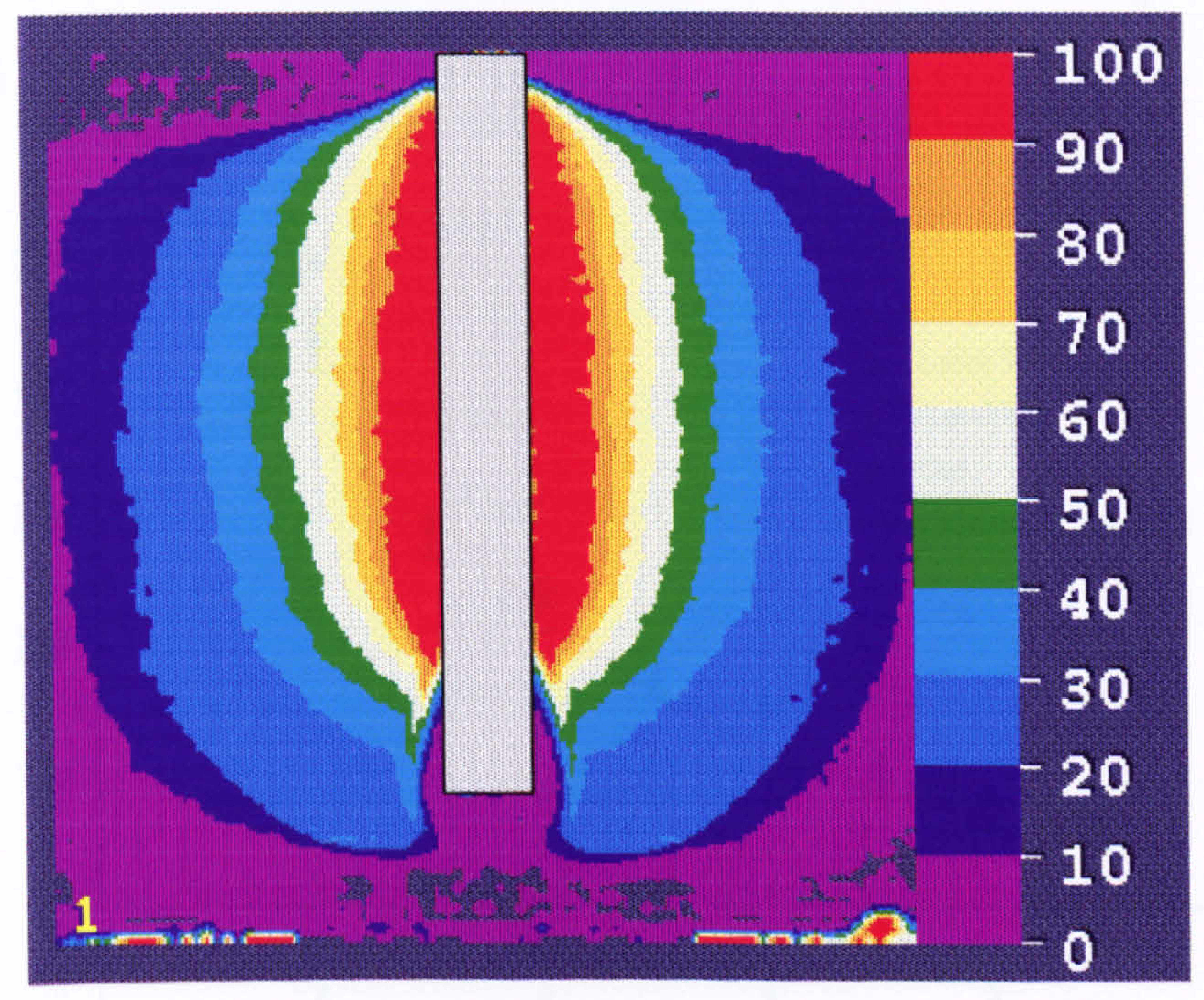

Figure 4.6. Typical normalized dose distribution of the irradiated gel. The scale shows the percentage of maximum dose. The image shows the sagittal plane. The channel in the middle of the image represents the position of the applicator 


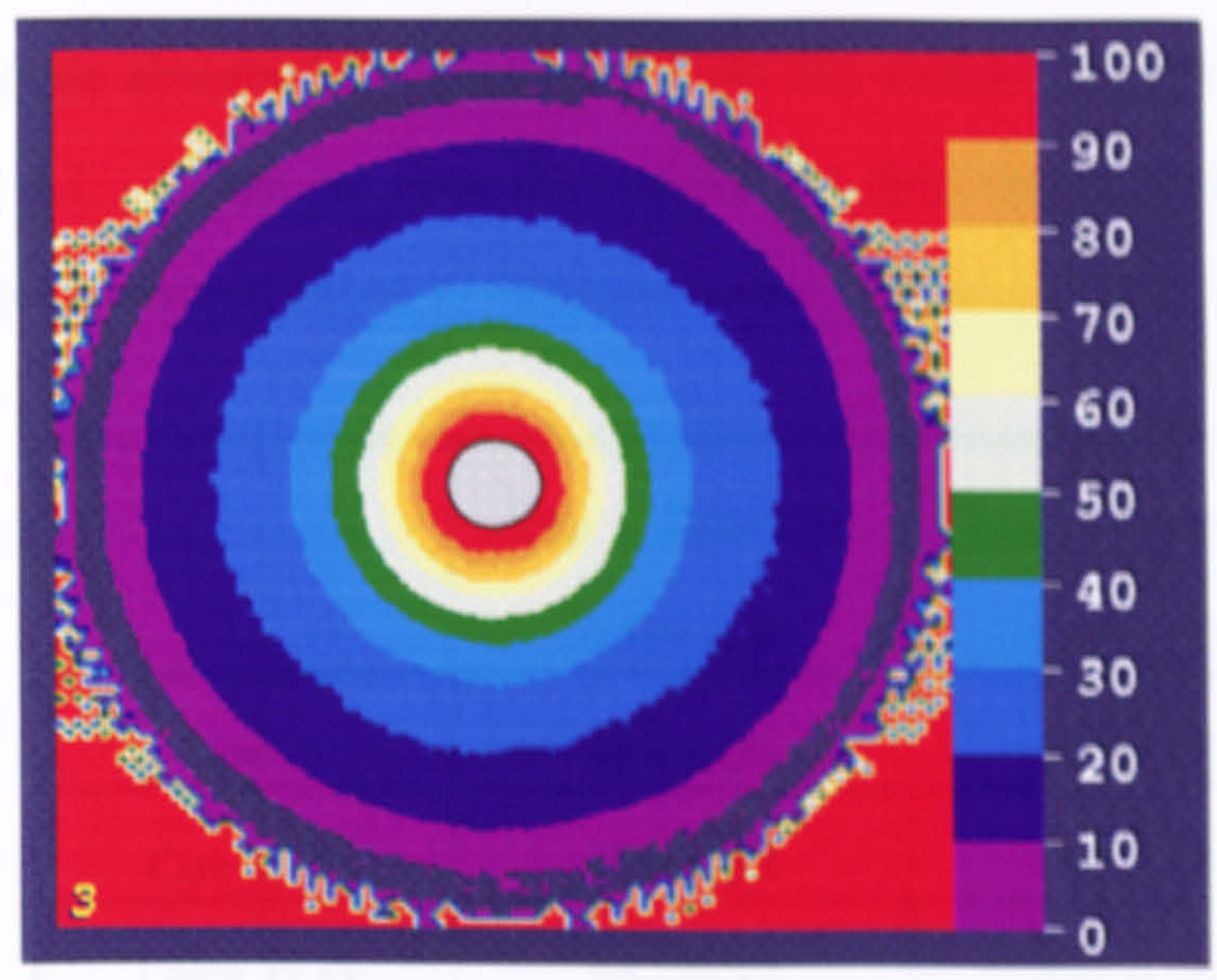

Figure 4.7. Isodose levels produced in the irradiated gel in the coronal plane

The results of absolute doserate comparison from 2 to $5.5 \mathrm{~cm}$ from the centre of the source train between gel, TLDs and calculation are shown in figure 4.8 for the first experiment. It is can be seen from the results that all four are in good agreement within $\pm 5 \%$.

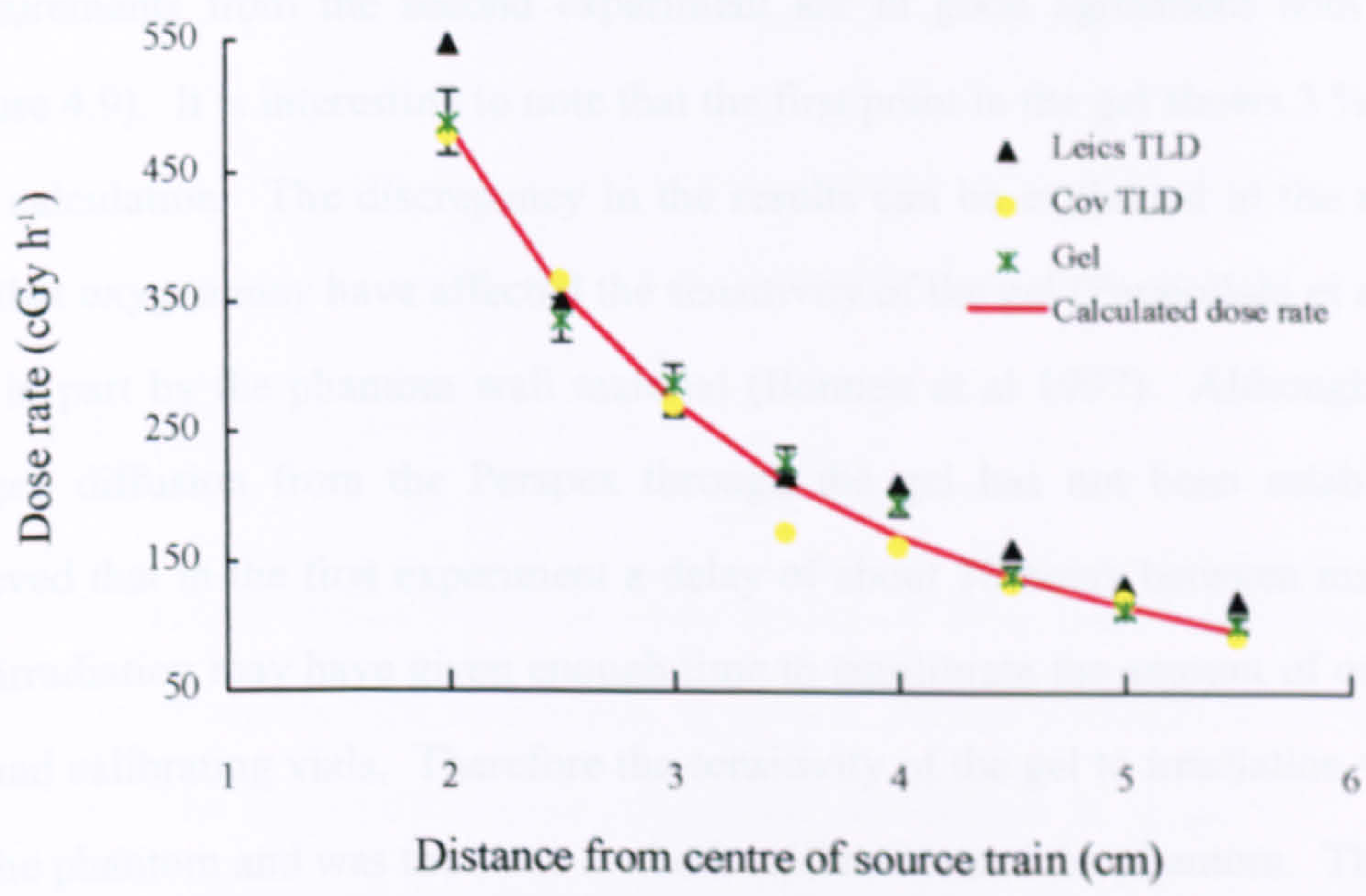

Figure 4.8. Absolute dose rate measured at different distances from the centre of the applicator. 
The absolute absorbed doserate $2 \mathrm{~cm}$ from the source for both experiments are shown in table 4.1. It can be seen that unlike the first experiment, in the second irradiation the gel measured about $17 \%$ higher dose than the calculation.

\begin{tabular}{|c|c|c||}
\hline & $\begin{array}{c}\text { Exp 1. Doserate } \\
\left(\mathrm{cGy} \mathrm{h}^{-1}\right)\end{array}$ & $\begin{array}{c}\text { Exp 2. Doserate } \\
\left(\mathrm{cGy} \mathrm{h}^{-1}\right)\end{array}$ \\
\hline \hline Gel & $488 \pm 24$ & $561 \pm 28$ \\
\hline Cov TLD & $479 \pm 19$ & $461 \pm 18$ \\
\hline Leics TLD & $547 \pm 32$ & $487 \pm 19$ \\
\hline Calc & 488 & 481 \\
\hline
\end{tabular}

Table 4.1. Comparison of the absolute doserate measured at $2 \mathrm{~cm}$ from the centre of the source for two different experiments

If however the dose is normalized to a depth of $3.5 \mathrm{~cm}$ the relative measurements from the second experiment are in good agreement with each other (Figure 4.9). It is interesting to note that the first point in the gel shows $3 \%$ lower dose than calculation. The discrepancy in the results can be explained in the main by the fact that oxygen may have affected the sensitivity of the gel (Farajollahi et al 1997) and also in part by the phantom wall material (Bonnett et al 1997). Although the rate of oxygen diffusion from the Perspex through the gel has not been established, it is believed that in the first experiment a delay of about 30 hours between manufacturing and irradiation may have given enough time to equilibrate the amount of oxygen in the gel and calibrating vials. Therefore the sensitivity of the gel to irradiation was reduced for the phantom and was the same for both calibration and the phantom. The oxygen in this experiment can diffuse throughout the Perspex insert as well as Perspex phantom wall. For the second experiment the absolute measurement of absorbed dose was 
was higher than that calculated by $17 \%$ possibly due to a different gel sensitivity as described (3.7.11). The time delay between manufacturing and irradiation for this experiment was about 4 hours. Also in the second experiment the relative dose $2 \mathrm{~cm}$ from the source train, where it is close to the Perspex insert, shows $3 \%$ less dose than the calculation which lends further support for effect of oxygen on gel sensitivity.

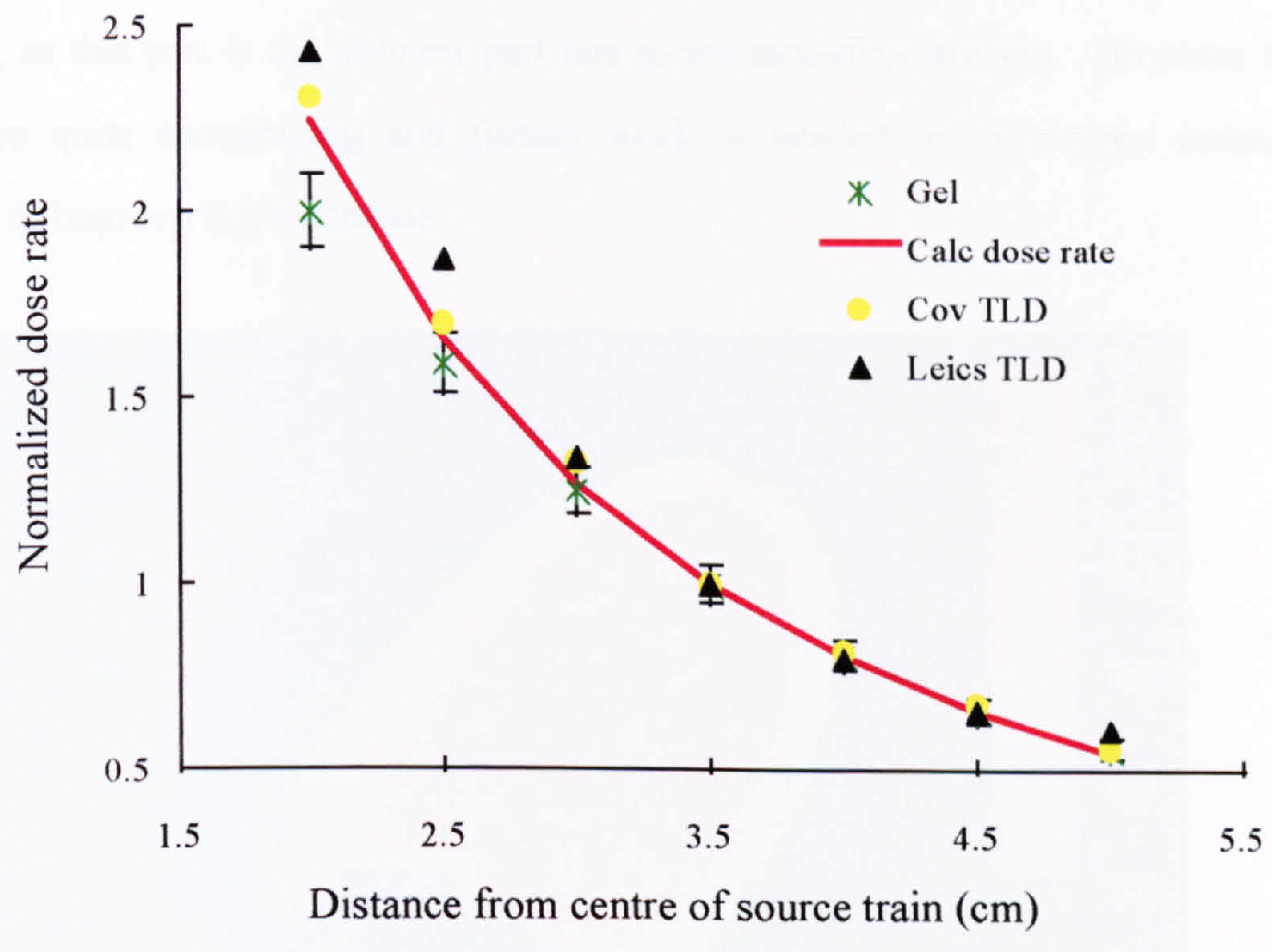

Figure 4.9. Relative dose normalized to $3.5 \mathrm{~cm}$ from the centre of the source train

\subsubsection{Complex brachytherapy}

Figure 4.10 shows the preliminary results of dose distribution produced in the gel for an inter uterine tube and ovoid. It is clear that no polymerization exists behind the ovoids. The results of isodose comparison are shown in figure 4.11. There is a good agreement between the gel and Helax-TMS in the isodose level of 100 to $50 \%$ to within less than $0.5 \mathrm{~mm}$. 
This preliminary experiment was intended to show how well the gel can be used in simulating complex geometries under real clinical conditions. As it was quite difficult to mould Perspex to accommodate the gynae applicator, PETG (the material used for patient immobilisation shell in radiotherapy) was used for the side of the phantom in contact with the applicator. The part of the gel which does not produce any polymerization behind the ovoid most likely results from increased oxygen diffusion, as this part is the thinnest part due to the moulding process. However the results are quite encouraging and further work is needed using oxygen resistant materials to improve this technique.

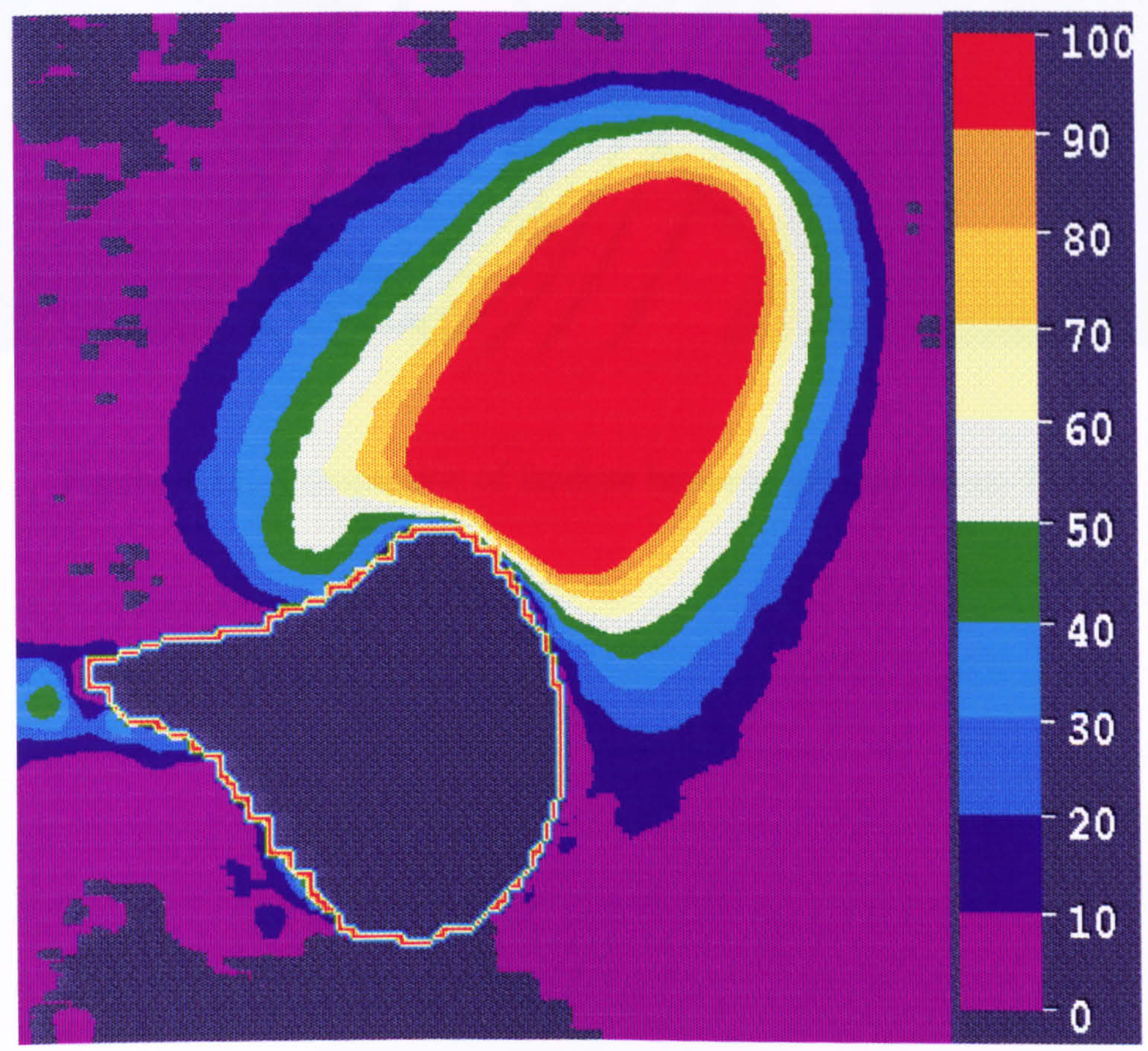

Figure 4.10. An image of the dose distribution produced using the Gynaecological applicator 


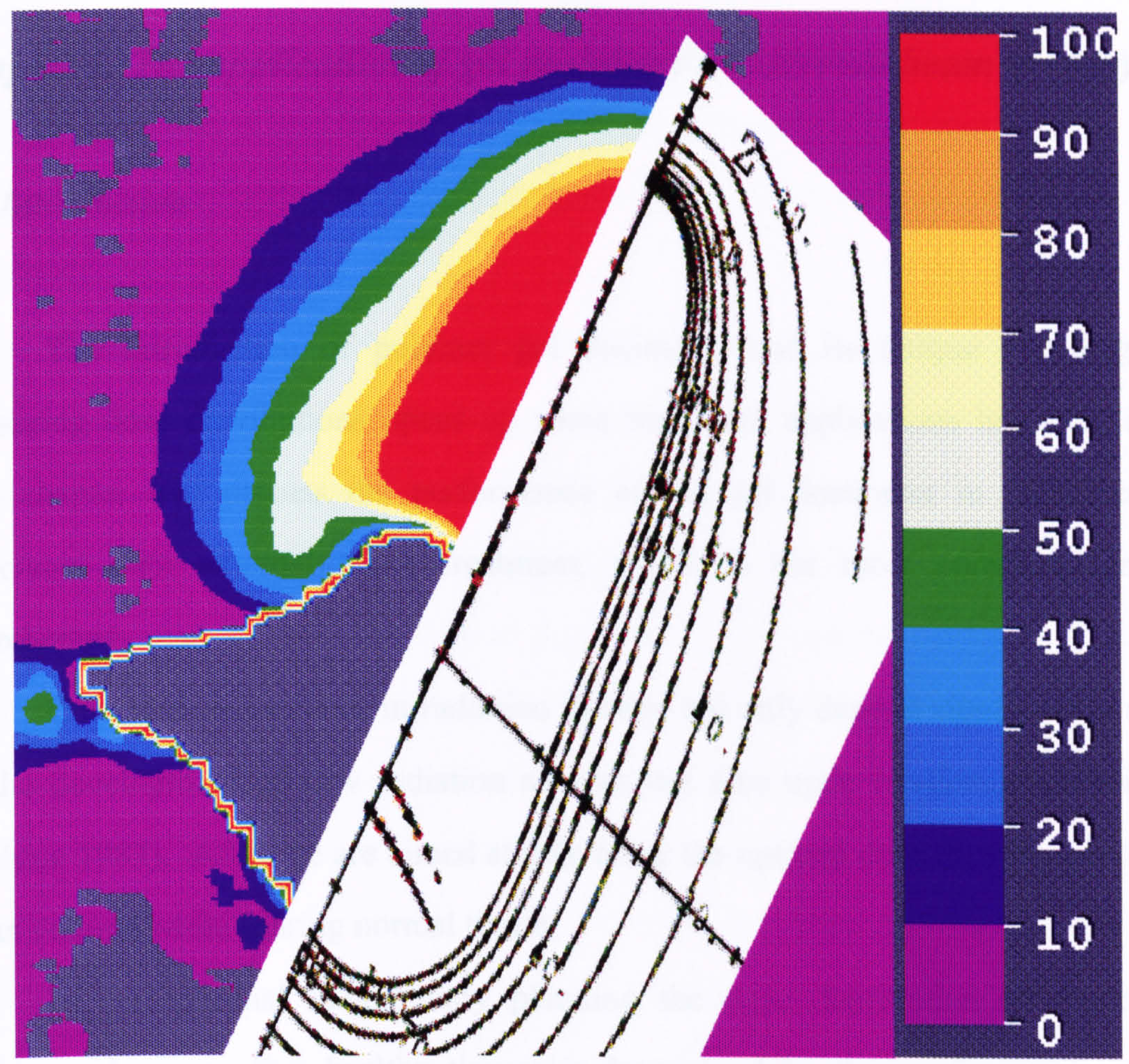

Figure 4.11. Comparison of isodose between gel and Helax-TMS 


\section{Chapter 5. The application of gel dosimetry in external beam therapy}

\subsection{Introduction}

The introduction of polymer gel dosimetry and its unique advantages in measuring dose distributions opens up some important applications in radiotherapy. This chapter will discuss the performance of the gel dosimeter in measurements associated with external beam treatment, which is the most common form of radiotherapy.

Current developments in radiation therapy not only depend significantly upon on the development of new radiation sources but also upon irradiation techniques (Brahme 1987). All these are aimed at delivering the optimal dose distribution to the target volume while sparing normal tissue.

In conventional radiotherapy planning the dose distribution in the target volume and surrounding healthy tissues is determined by the configuration of the incident beams. A recent development has been the introduction of inverse radiotherapy planning. For this technique the method of traditional treatment planning is reversed. Optimal incident beam profiles are determined to ensure that normal tissues receive the lowest possible damage for a given dose to the target volume (Brahme et al 1990). Conventional techniques are rather simple and can in many cases produce the desired dose distribution with sufficient sparing of normal tissue and clinical situations, so there is no requirement for complex plans. Inverse treatments are usually required where there are several organs at risk and the target volume itself is complex (Lind and Källman 1990).

In order to implement inverse radiotherapy planning in patient treatments, non uniform dose delivery is required to shape the dose distribution in an optimal manner to the target volume. In order to achieve the optimal shape of the incident beam, intensity modulated radiation treatment (IMRT) is employed using either customized compenstors or dynamic multi-leaf collimators (DMLC). 
The compensators are designed to shape the radiation beam profile required by the treatment plan. In order to make the manufacturing fast and accurate the compensators are usually made using computer numerically controlled (CNC) milling machines.

In the DMLC technique the collimator leaves move during dose delivery to produce the non-uniform dose distribution required by the treatment plan. This can be either continuous or segmented i.e. the so called "stop and shoot" technique.

A special case of IMRT is the "dynamic wedge" (Kijewski et al 1978). This generates and delivers a wedge-shaped dose profile by sequential movement of one independent collimator jaw. The usual method of achieving wedge-shaped dose distribution is to place a metal wedge shaped filter in the radiation beam. In using the dynamic wedge there is no need to use an external beam modifier, as the movement of one computer controlled collimator jaw creates a continuously variable field of decreasing widths. For this reason a standard field scanner cannot be used for the measurement of isodose curves. Therefore it requires an alternative method of dosimetry that will integrate the dose at each point during the entire dose delivery (Bidmead et al 1995). Several investigators have used different dosimetric methods to measure dose distribution for the dynamic wedge. For example diode detector arrays, radiation sensitive film, Fricke gel dosimeters and ion chamber arrays (Bengtsson et al 1996, Liu et al 1997 and Zhu et al 1997).

In general IMRT techniques have the potential to spare critical organs near the target volume whilst achieving good tumour dose distributions for complex geometries. The disadvantages of these methods are;-

they are time consuming,

need good patient immobilisation,

patient breathing and organ motion may need to be taken into account. verification of the dose distribution is difficult.

Implementation of these techniques require more complex treatment planning methods and consequently demand reliable integrating dosimeters that are capable of 
measuring complex dose distributions in 3-D with high spatial resolution. The polymer gel dosimeter has been shown to fulfil these requirements (Chapters 3,4).

The aim of this chapter is to demonstrate the performance of BANG polymer gel in both conventional therapy and intensity modulated beam therapy. In order to do this central axis depth doses have been measured and compared with the results of ion chamber measurements. Dose distributions from three, four and oblique beam treatments were compared using the Helax-TMS computer planning system. Dose distributions for IMRT were produced using customized filters and compared with both calculations and measurement using film. Finally the dose distribution from a $45^{\circ}$ dynamic wedge was determined.

\subsection{Single field irradiation (Depth dose determination)}

A cylindrical Perspex container of $150 \mathrm{~mm}$ in diameter, $190 \mathrm{~mm}$ in height with wall thickness of $3 \mathrm{~mm}$ was filled with 2.5 litres of BANG gel. In order to obtain a central axis depth dose curve the gel phantom was irradiated through the base of the cylinder using a $6 \mathrm{MV}$ x-ray beam and a radiation field of $5 \times 5 \mathrm{~cm}^{2}$. The measurement was repeated with field size of $8 \times 8 \mathrm{~cm}^{2}$ and the results compared with data measured using a small ionization chamber. A dose of $8 \mathrm{~Gy}$ was given at a depth of $1.5 \mathrm{~cm}$ in both measurements.

The dose distributions were measured using a $3 \mathrm{~mm}$ thick slice in the case of 5 $\times 5 \mathrm{~cm}^{2}$ field and a $20 \mathrm{~mm}$ slice for the $8 \times 8 \mathrm{~cm}^{2}$ field. Both slices were taken parallel to the beam axis (sagittal plane) in the gel. Increased slice thicknesses were used in the second experiment to improve SNR.

Measurements using the ion chamber were made in the same phantom using the same irradiation conditions. In this case the phantom was filled with water. The equipment constructed to make the measurements consisted of two lengths of Perspex rod joined together at right angles as shown in figure 5.1. The ionization chamber, Scanditronix RK which connected to RDM-2A Therados electrometer was mounted on 
the scaled section that was able to move either vertically or horizontally. The ion chamber was then irradiated at different depths along the central axis of the phantom.

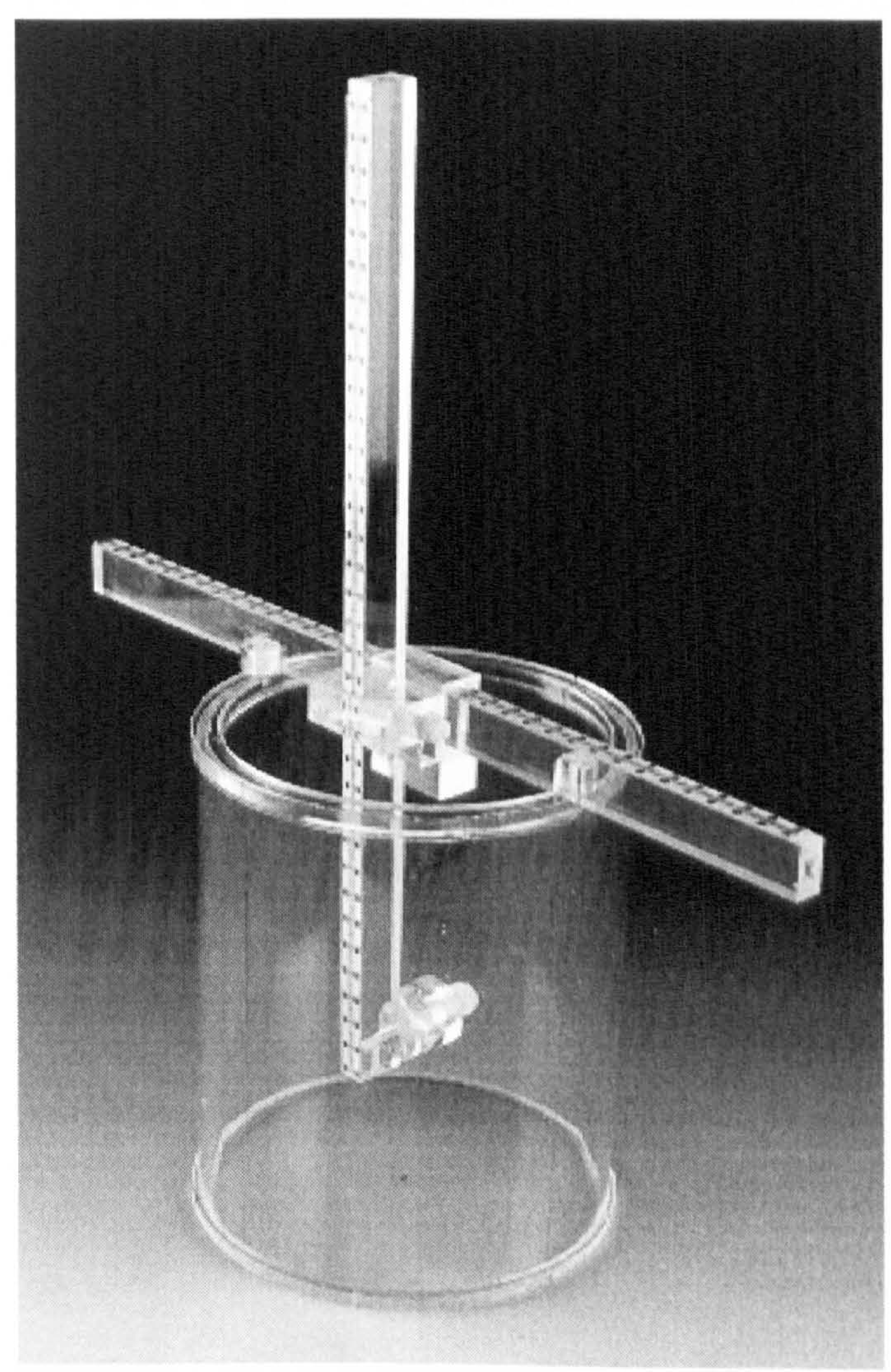

Figure 5.1. Experimental set up for ion chamber measurement

\subsubsection{Results}

The dose distribution resulting from the conversion of the calculated $\mathrm{T}_{2}$ image to dose using calibration data is shown in Figure 5.2. Figure 5.3 shows the dose profile taken from $\mathrm{d}_{\max }$ for the $8 \times 8 \mathrm{~cm}^{2}$ field. The percentage depth doses on the central axis 
derived from the dose distribution for field size of $5 \times 5 \mathrm{~cm}^{2}$ and $8 \times 8 \mathrm{~cm}^{2}$ are shown in figure 5.4 and 5.5 respectively. The build up region for the ion chamber measurements is not shown because of the constraints of the chamber holder.

The amount of error in ionisation chamber measurements (i.e. the variation in dose due to variations in chamber position) was less than $\pm 1 \%$ as indicated in figures 5.4 and 5.5. It is clear from the results that there is good agreement with the gel measurements for single fields. It is also clear from the figure 5.5 that the thicker slice resulted in improved SNR.

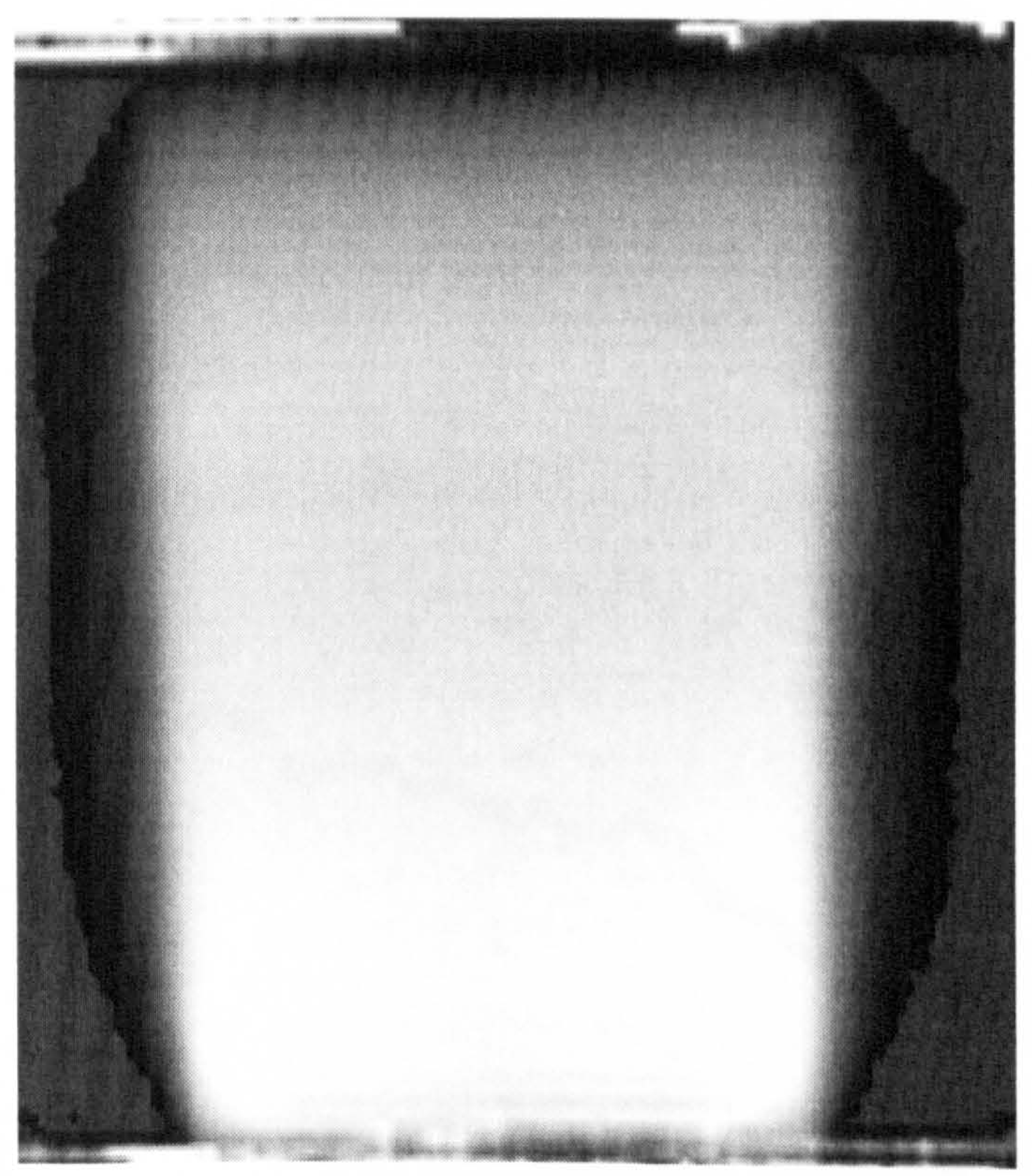

Figure 5.2. Dose distribution of single $8 \times 8$ radiation in the gel 


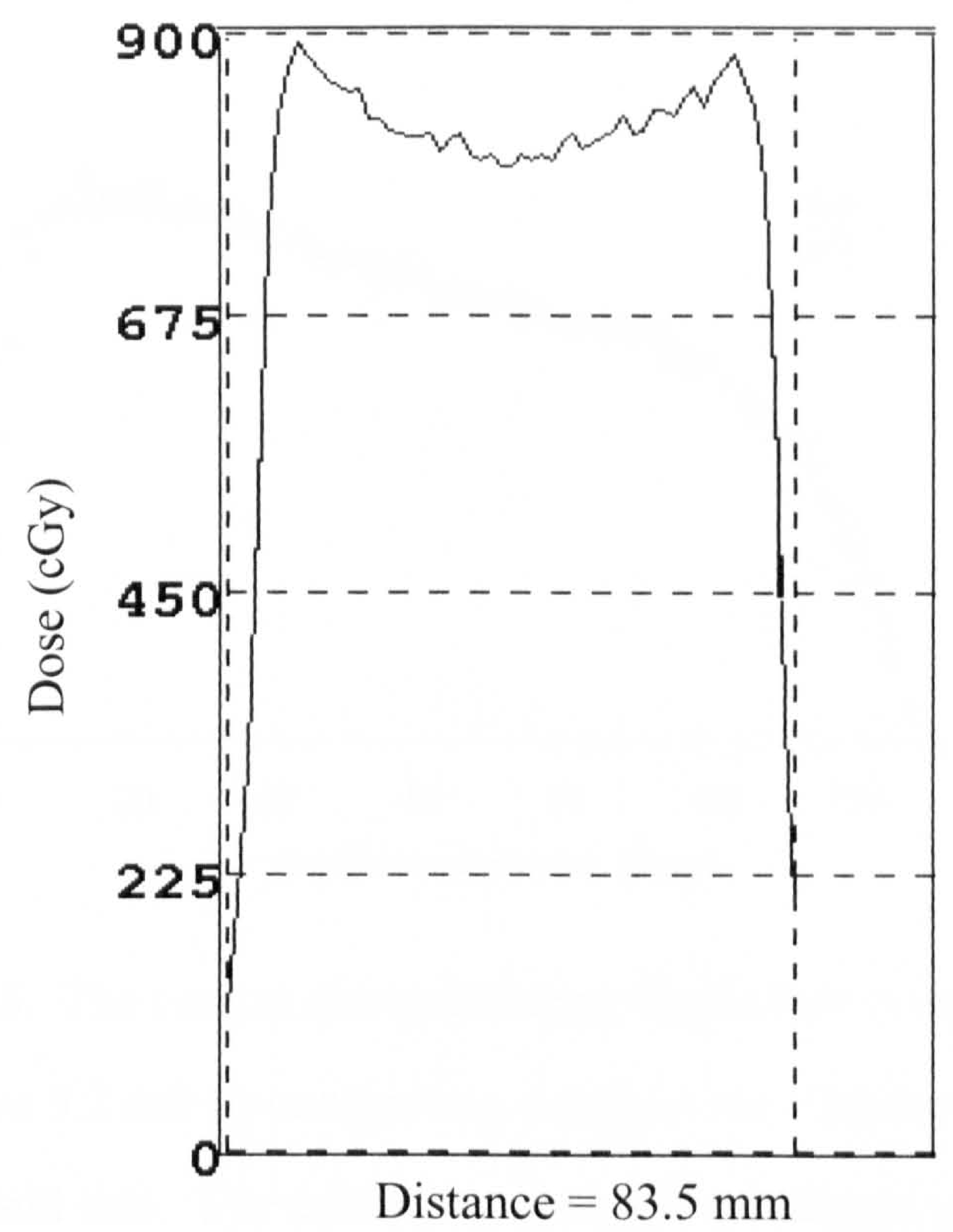

Figure 5.3 Horizontal profile taken at approximately $\mathrm{d}_{\max }$ in the gel irradiated by $8 \times 8 \mathrm{~cm}^{2}$ radiation field

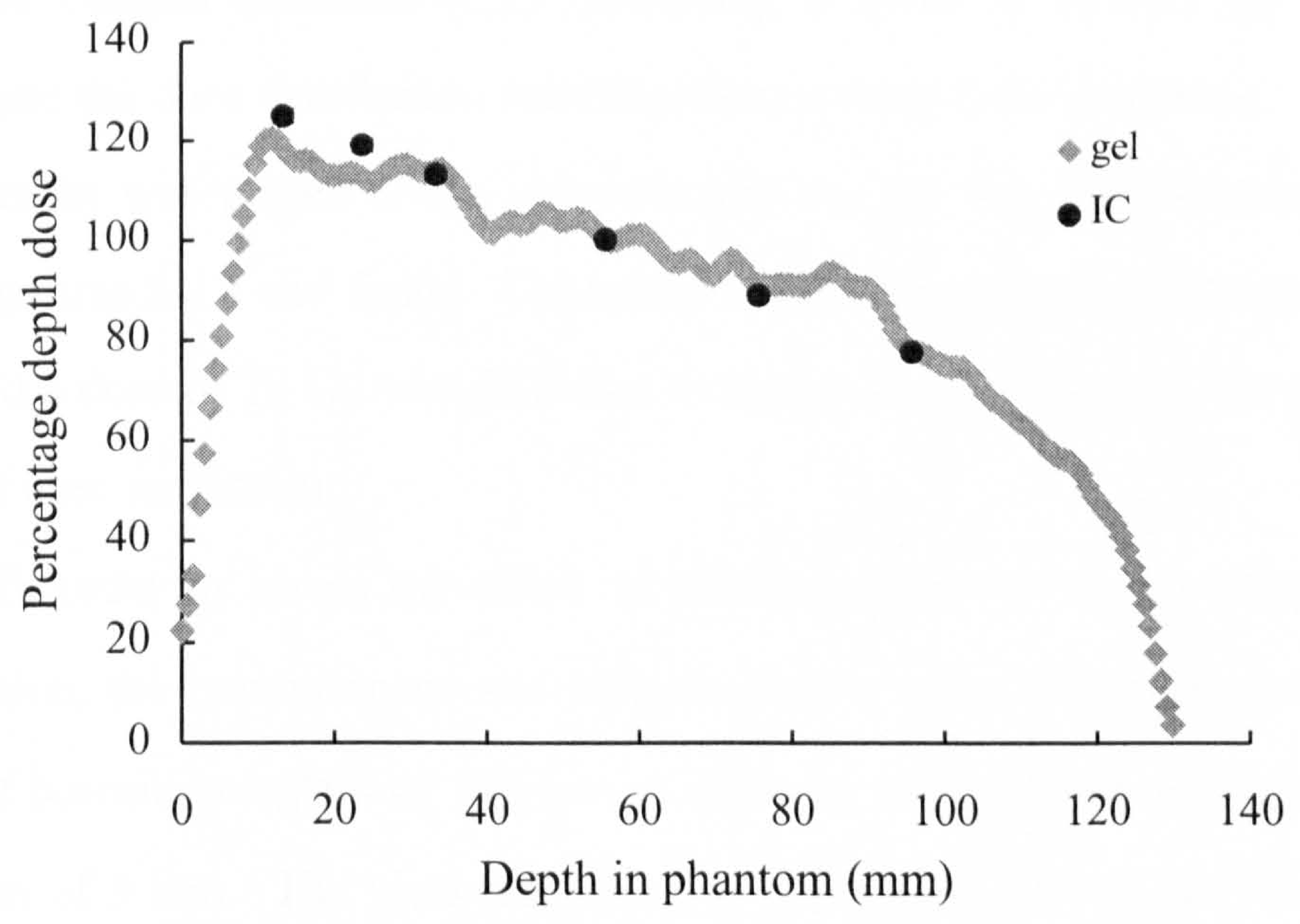

Figure 5.4. The comparison of a central axis percentage depth dose curve for $5 \times 5 \mathrm{~cm}^{2}$ field size using ion chamber and gel dosimeter. The data are normalized to a depth of $5 \mathrm{~cm}$ 


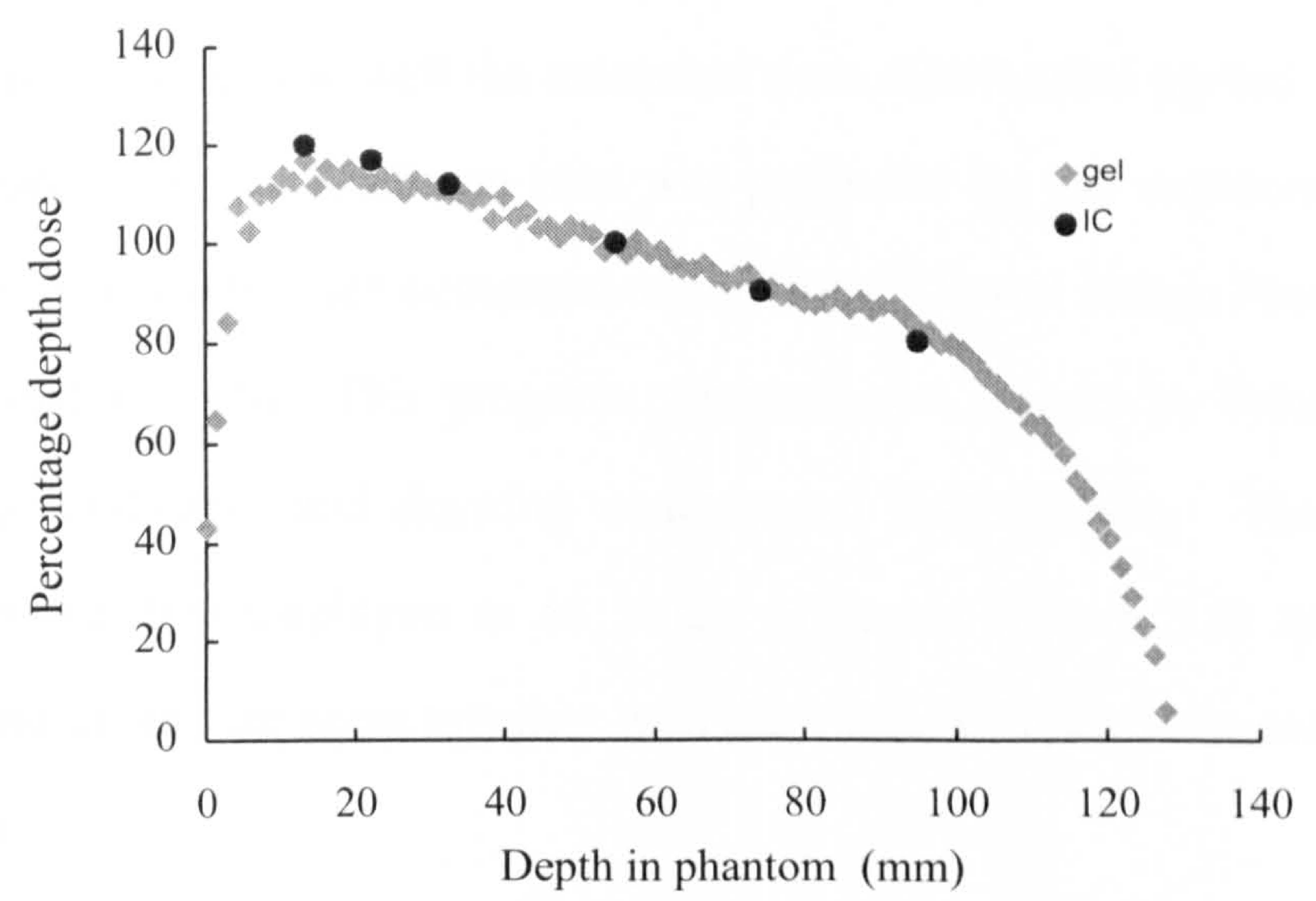

Figure 5.5. The central axis percentage depth dose curve derived from figure 5.2 and its comparison using an ion chamber for $8 \times 8$ $\mathrm{cm}^{2}$ field size. The data are normalized to a depth of $5 \mathrm{~cm}$

\subsection{Three field irradiation}

A Perspex phantom (5.2) containing 2 litres of BANG gel was used to investigate the dose distribution resulting from a three field irradiation. The centre of the phantom was placed at the isocentre and the gel was irradiated horizontally by applying three $5 \times 5 \mathrm{~cm}^{2}$ fields. The radiation fields were separated from each other by $120^{\circ}$ and a dose of 10 Gy was delivered to isocentre i.e. giving 4.2 Gy per field at the depth of dose maximum.

In order to assess the effect of phantom material on the shape of the dose distribution, the measurement was repeated with a glass walled phantom which was made of borosilicate glass of $152 \mathrm{~mm}$ in diameter and $192 \mathrm{~mm}$ in height, with a wall thickness of $3 \mathrm{~mm}$. The glass phantom was almost identical to the Perspex phantom (5.2) and the time delay from manufacture to irradiation was the same for both measurements. In each case the gels were stored in a fridge and scanned four days after radiation with slice thickness of $20 \mathrm{~mm}$. The gel temperature was kept constant at $4^{\circ} \mathrm{C}$ for all the measurements. 
To investigate how well the measured dose distribution agreed with the HelaxTMS planning system a treatment plan was produced for the experimental condition. The isodose levels were then compared using "Pips" (Portal Image Processing System) software (Shalev 1996). This program compares two images by introducing fiducial points as a landmarks and drawing contours on both images. The results of the comparison are then displayed as an image of regret (figure 5.6) which shows the overdose and underdose areas together with the values of these area and the percentage differences.

\subsubsection{Results}

Figure 5.7 shows a typical dose distribution produced in the gel by three equally angled beams. Comparison of the measured dose distribution with Helax-TMS is shown in figure 5.8. For $50 \%$ isodose a comparison of dose areas shows an agreement to within $\pm 4 \%$ using Pips. The $20 \%$ isodose area in the gel close to the beam exit, is shallower than the calculation.

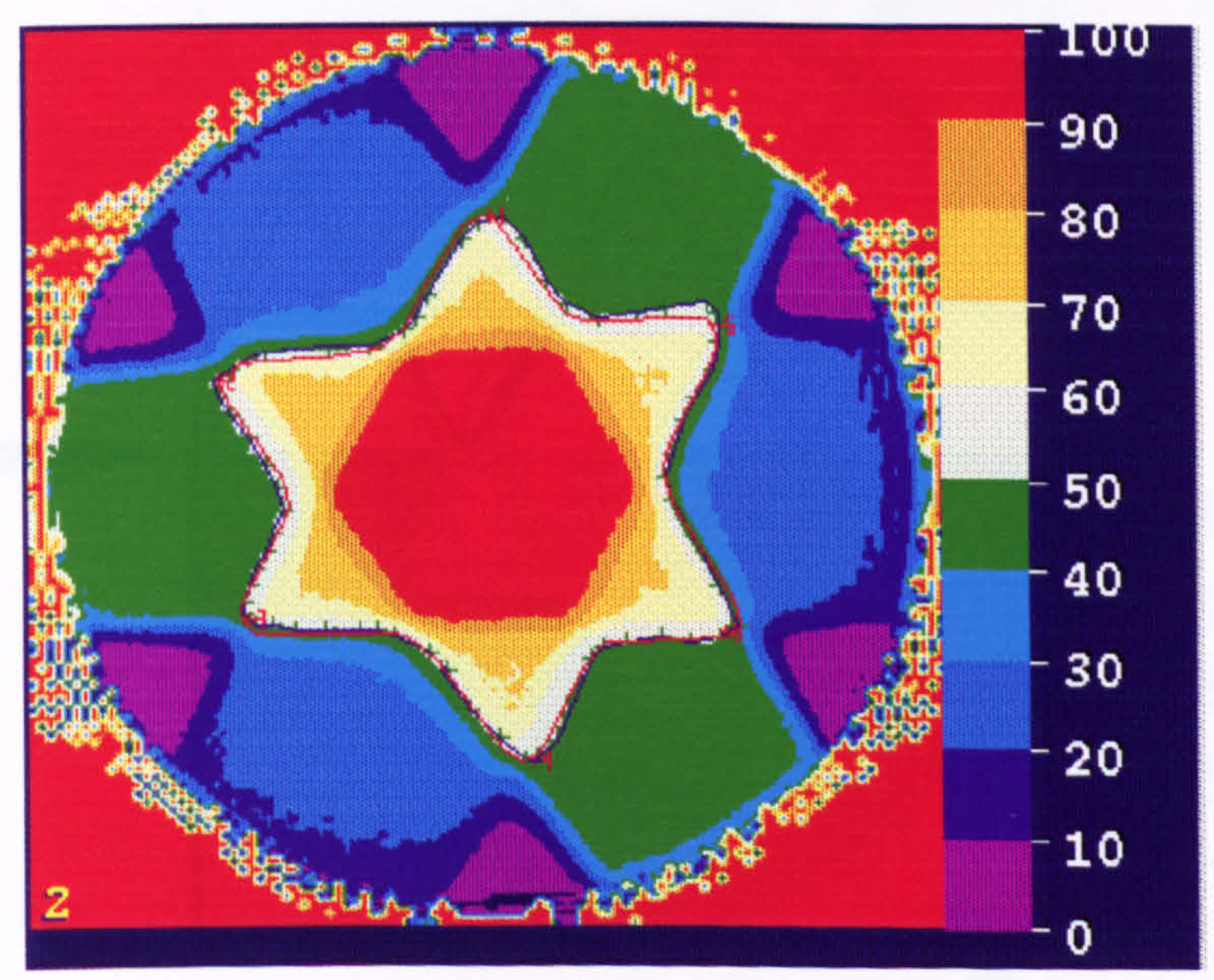

Figure 5.6. A typical image of regret produced using Pips. The scale represents the percentage of the dose at isocentre 


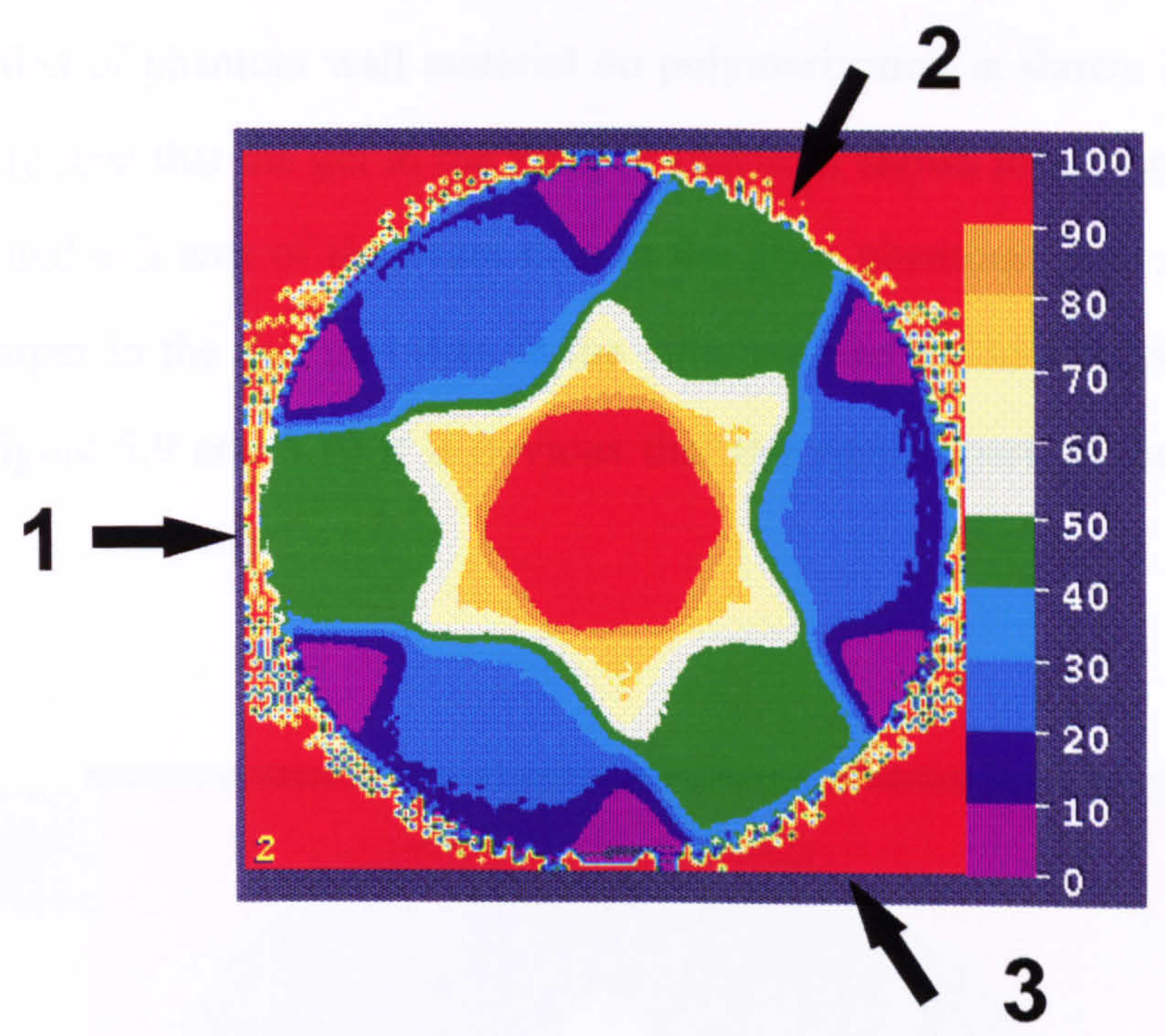

Figure 5.7. A typical dose distribution resulted from three field treatment using BANG gel in a glass walled phantom. The scale represents the percentage of the normalized value.

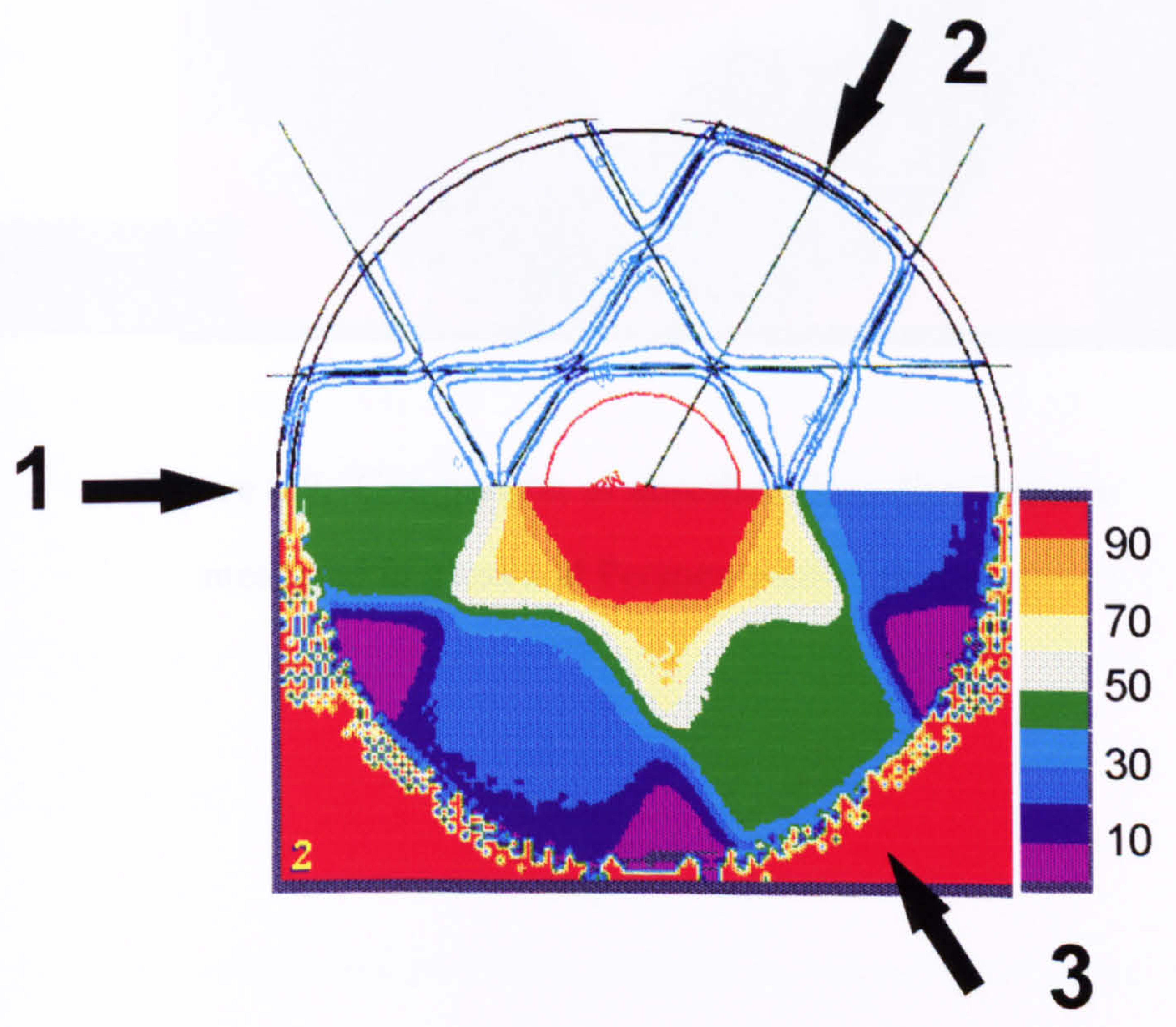

Figure 5.8. Comparison of a three field treatment plan between Helax-TMS and the polymer gel 
The effect of phantom wall material on polymerization is shown in figure 5.9. It can be clearly seen that the gel in the Perspex phantom shows lower isodose level in both entrance and exit area of the beam than in the glass phantom. The magnitude of the effect is larger in the exit part than in the entrance part as demonstrated in figure 5.10. From figure 5.9 and 5.10 it is obvious that the central part of the gel remains unaffected.

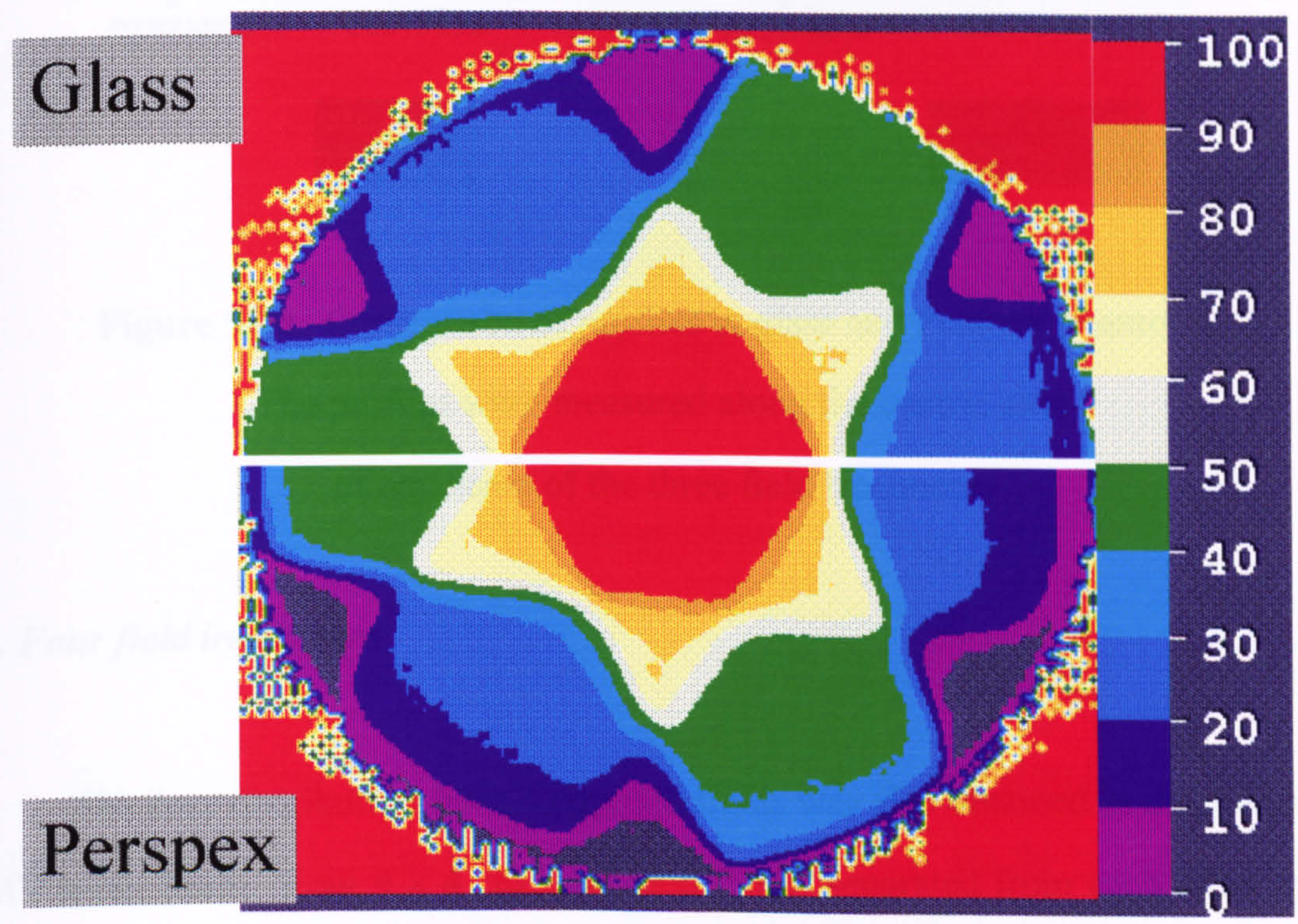

Figure 5.9. Comparison of absorbed dose distributions measured in glass and Perspex walled phantom. 


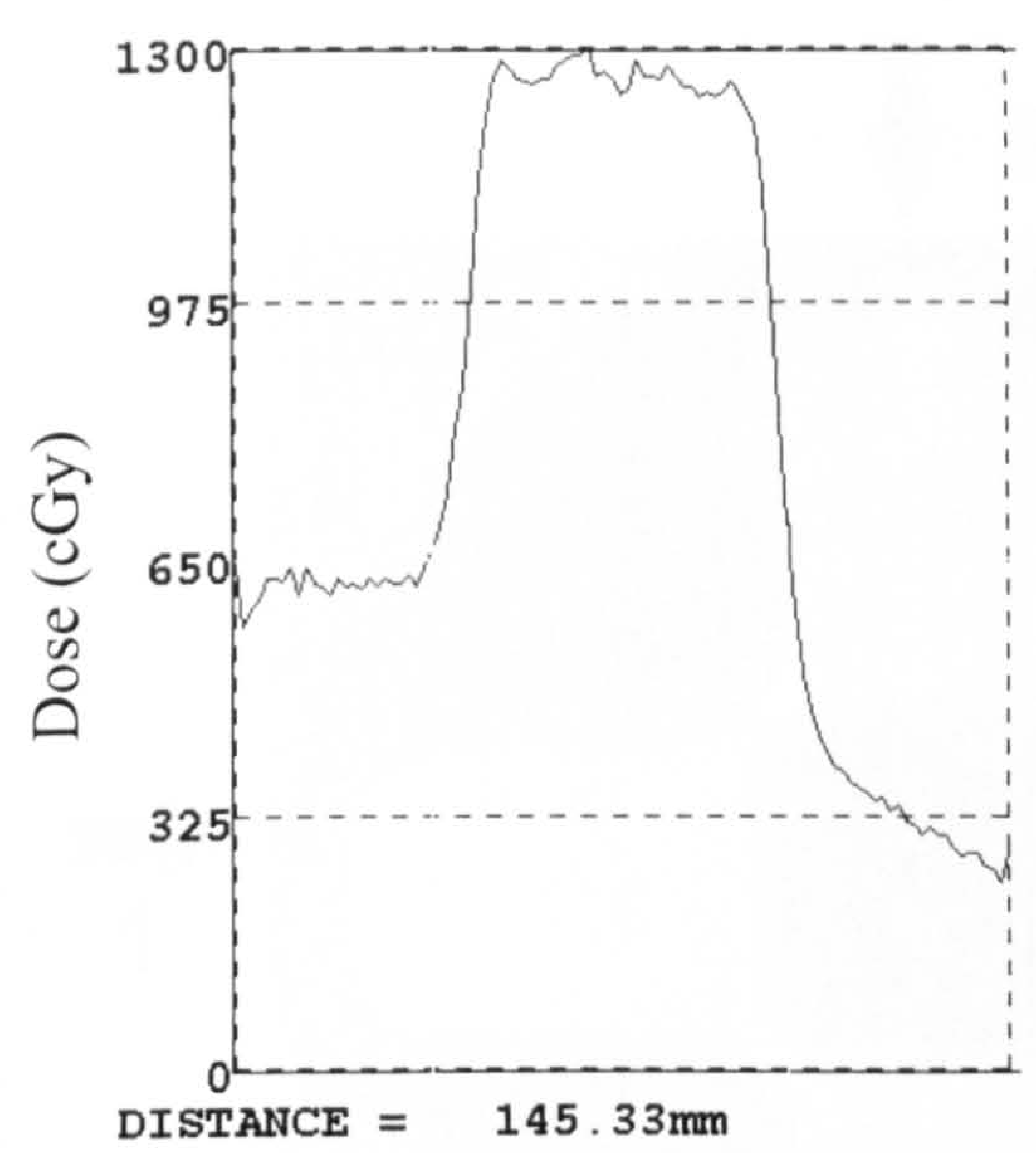

Glass

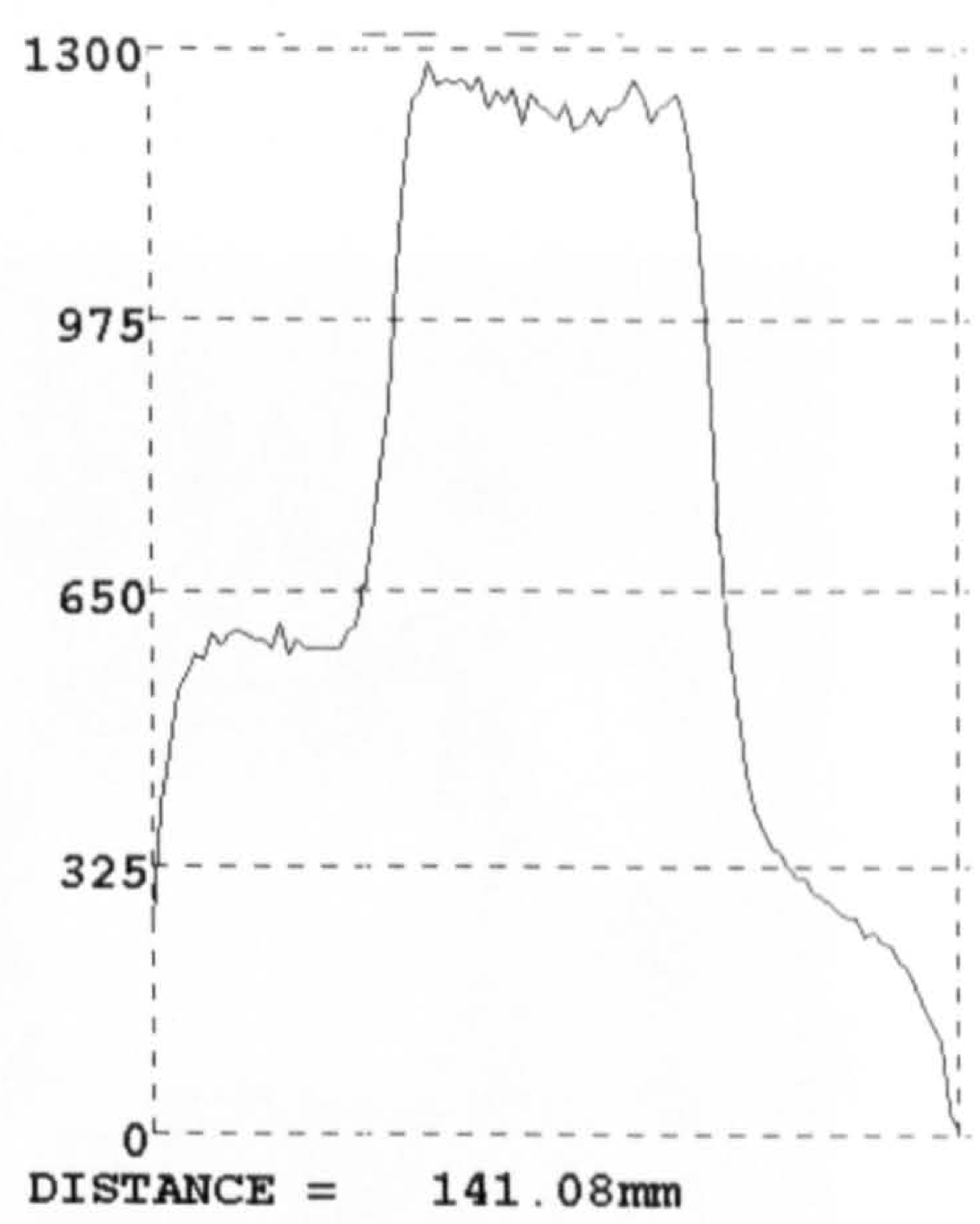

Perspex

Figure 5.10. Comparison of profiles in glass and Perspex phantoms.

The profiles were measured along the central axis

of one beam of the three field treatment.

\subsection{Four field irradiation}

The dose distribution from 4 opposed fields was also obtained in the gel using a $6 \mathrm{MV}$ x-ray beam. Four $4 \times 4 \mathrm{~cm}^{2}$ radiation fields, separated from each other by $90^{\circ}$ were applied at the centre of a 2 litre gel phantom. The total dose at the isocentre was calculated to be $10 \mathrm{~Gy}$. The gel was scanned four days after radiation and a coronal slice of $20 \mathrm{~mm}$ thick was taken parallel to the beam axis. The dose distribution measured by the gel was compared with a plan produced using the Helax-TMS.

\subsubsection{Results}

The result of the absorbed dose distribution measurement using the four field treatment (two sets opposed fields) is shown in figure 5.11. This figure demonstrates the square region of high dose in the intersection of all four fields. 


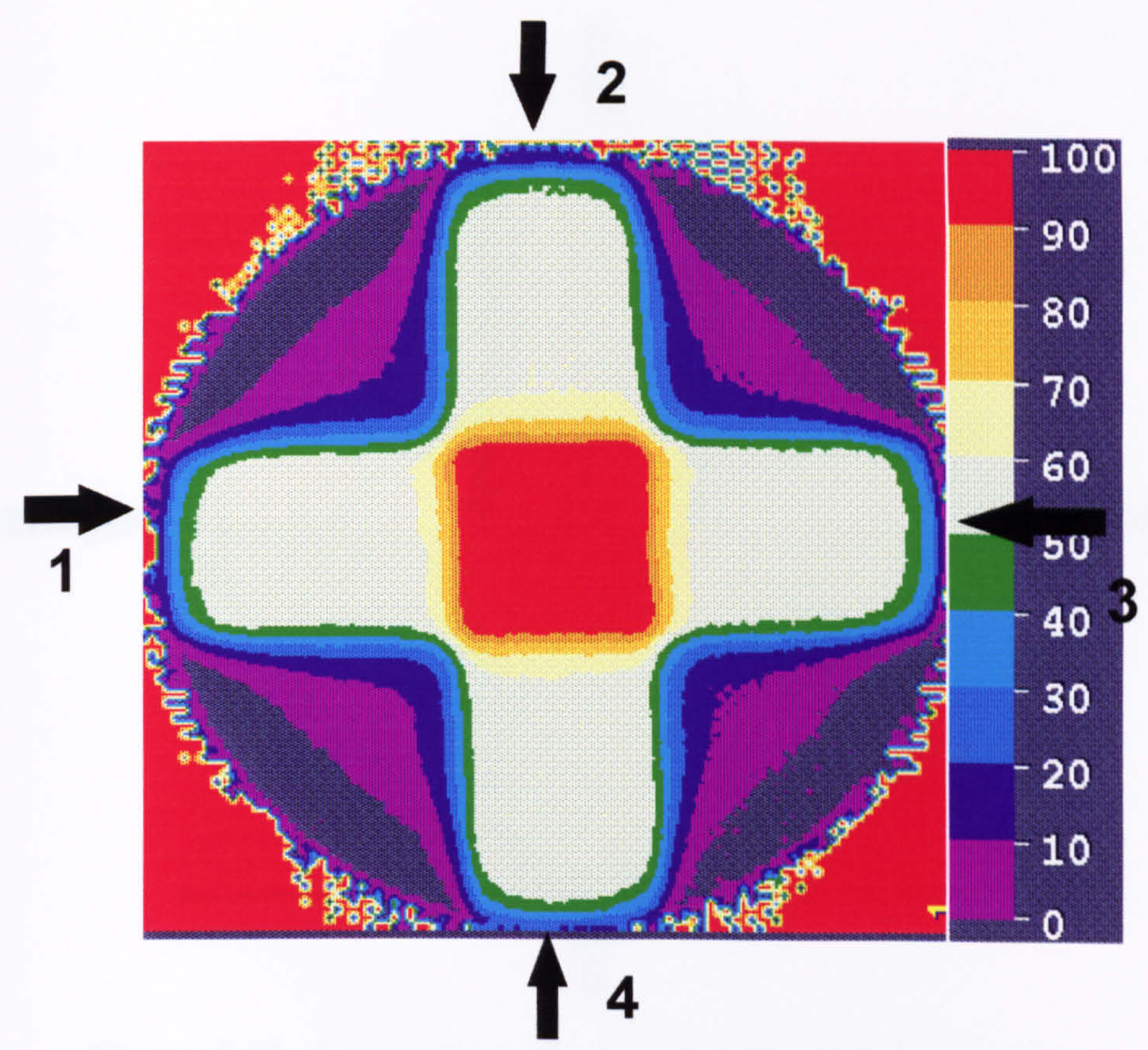

Figure 5.11. The MRI produced dose map in the gel irradiated to four field treatment

The comparison of the isodose curve using Helax-TMS and the polymer gel (figure 5.12) resulted in agreement better than $3.5 \mathrm{~mm}$ from the $90 \%$ to $60 \%$ isodose levels. The comparison of the dose area that was made by Pips resulted in agreement of \pm 2.7 to $5 \%$ up to isodose level of $70 \%$ and $\pm 8 \%$ at $60 \%$. The $50 \%$ isodose area in the gel is shallower than the Helax-TMS as was expected because of being close to the Perspex wall. 


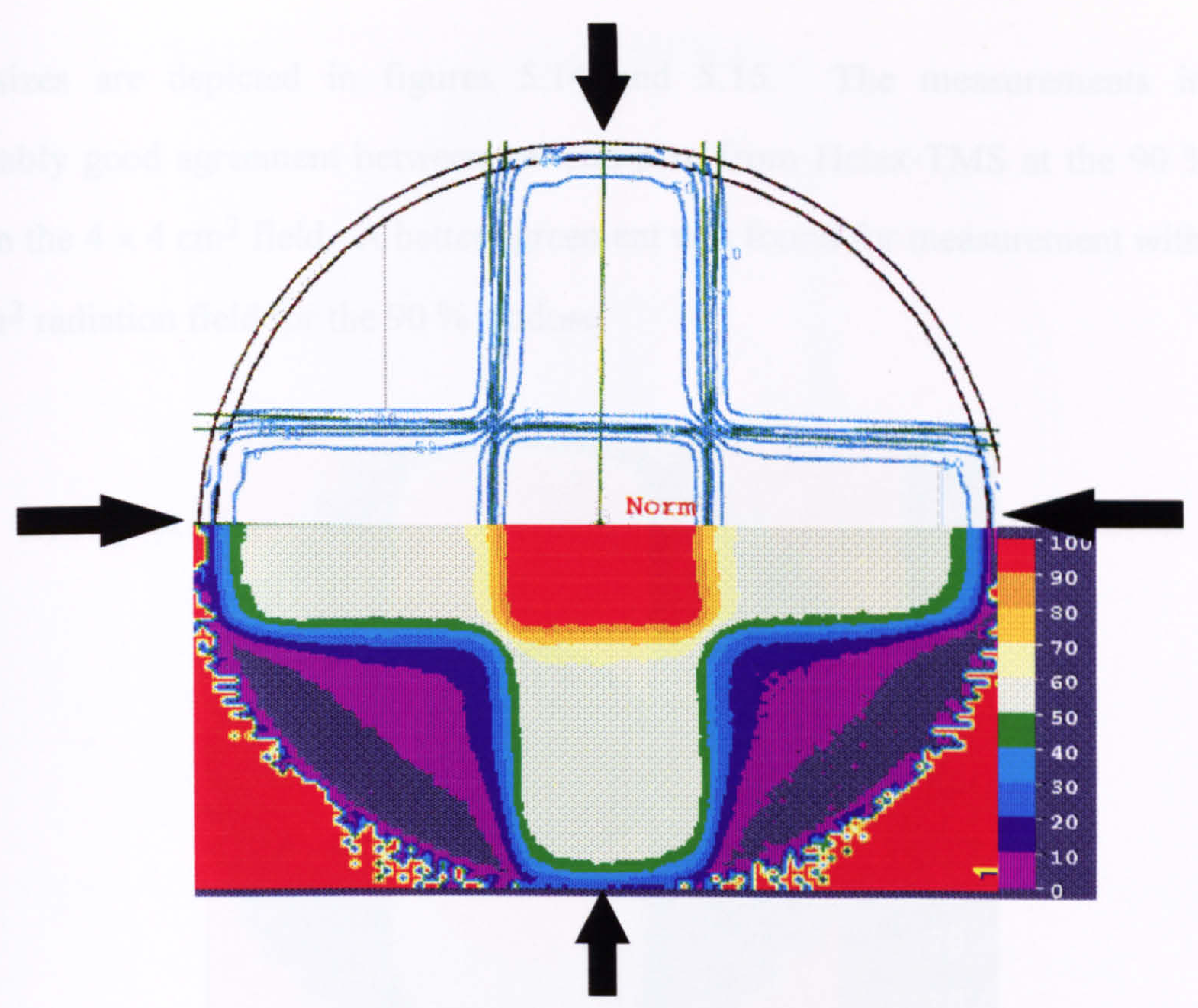

Figure 5.12. Comparison of a four field treatment plan using Helax-TMS with measurement with a polymer gel.

\subsection{The dose distribution in an inhomogeneous gel}

In order to create an inhomogeneity inside the gel, a Perspex tube of $5 \mathrm{~cm}$ diameter and $15 \mathrm{~cm}$ high with wall thickness of $3 \mathrm{~mm}$ was placed centrally inside the phantom. Two measurements were made using $4 \times 4$ and $6 \times 6 \mathrm{~cm}^{2}$ fields. The former was irradiated using a $6 \mathrm{MV}$-ray beam and the latter an $8 \mathrm{MV}$ beam with the horizontal beam. A dose of $8 \mathrm{~Gy}$ was delivered to a depth of $1.5 \mathrm{~cm}$ and $2 \mathrm{~cm}$ i.e. the depth of dose maximum $\left(\mathrm{d}_{\max }\right)$ respectively. The isodose levels from the planned distributions using Helax-TMS were compared with the dose distributions measured in the gel.

\subsubsection{Results}

Figure 5.13 shows a dose distribution produced with a inhomogeneity in the gel; in this case an air gap. The comparison of isodose levels for different radiation 
field sizes are depicted in figures 5.14 and 5.15. The measurements indicate reasonably good agreement between gel and plan from Helax-TMS at the $90 \%$ dose level in the $4 \times 4 \mathrm{~cm}^{2}$ field. A better agreement was found for measurement with the 6 $\times 6 \mathrm{~cm}^{2}$ radiation field for the $90 \%$ isodose.

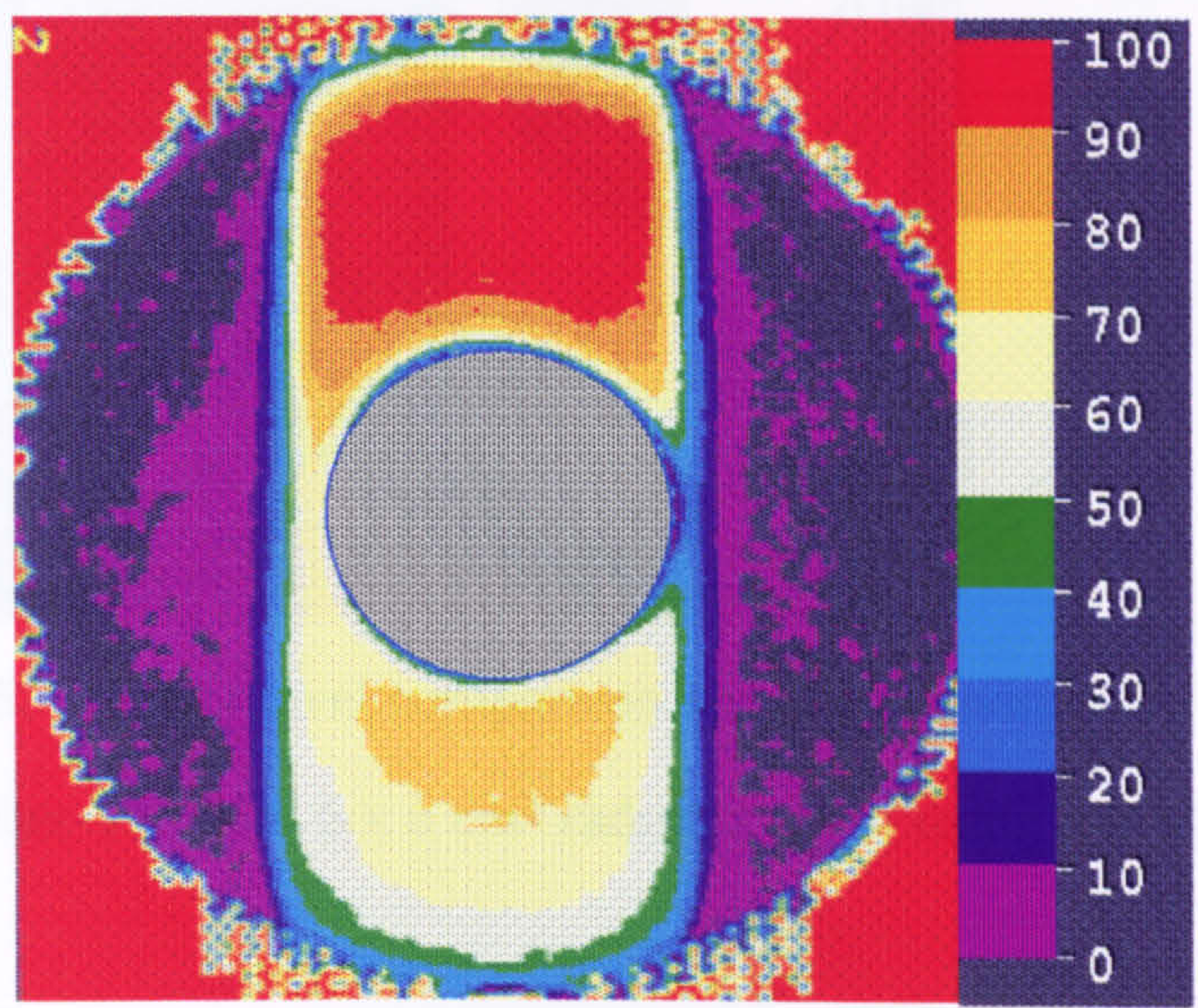

Figure 5.13. A typical dose distribution resulted in the irradiated gel with an air inhomogeneity inside for $6 \times 6 \mathrm{~cm}^{2}$ radiation beam. Note that there is a slight offset between the beam and the air-filled central cylinder.

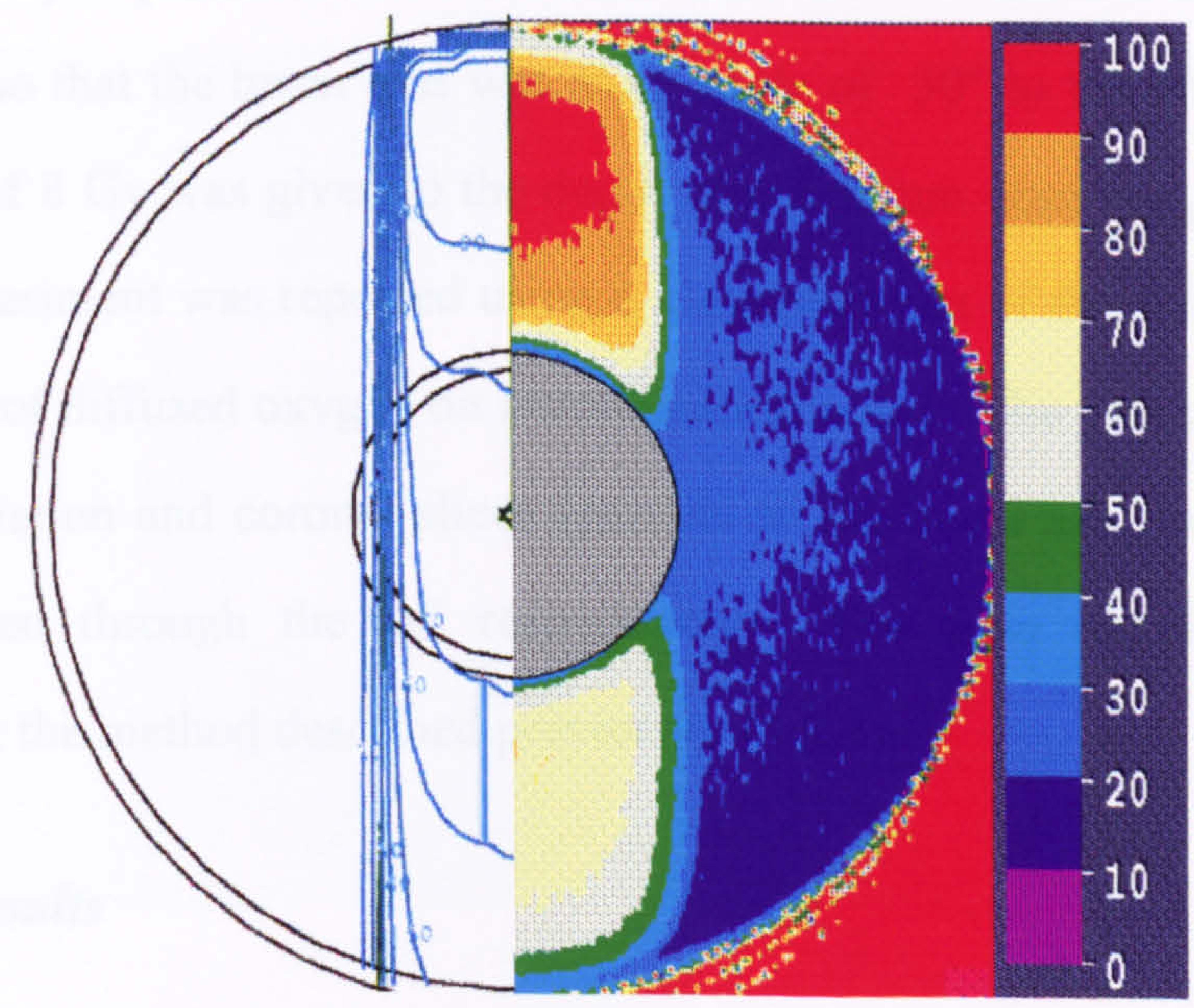

Figure 5.14. Comparison of dose distribution produced in the gel with an air gap included and Helax-TMS for $4 \times 4 \mathrm{~cm}^{2}$ field irradiated using a $6 \mathrm{MV}$ beam 


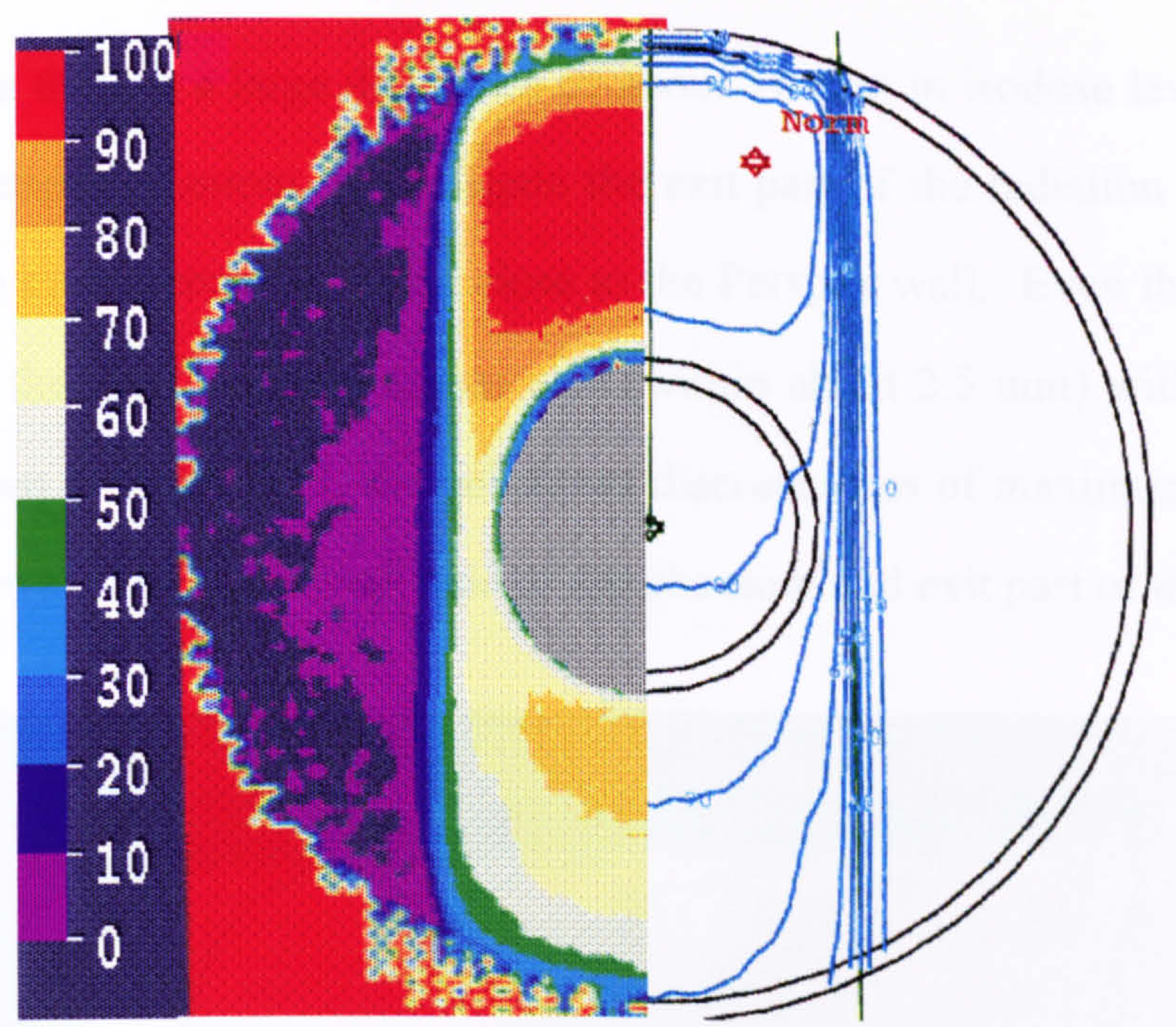

Figure 5.15. Isodose level comparison using gel having an air gap inside and Helax-TMS for $6 \times 6 \mathrm{~cm}^{2}$ radiation field using a $8 \mathrm{MV}$ beam

\subsection{Oblique beam}

A measurement of dose distribution produced by an oblique beam was obtained using a Perspex phantom and compared with a treatment plan. 2 litres of gel was prepared in a Perspex phantom. A horizontal beam of $4 \times 4 \mathrm{~cm}$ was applied parallel to the gel surface so that the beam axis was at an angle of $30^{\circ}$ to the phantom wall. An absorbed dose of $8 \mathrm{~Gy}$ was given to the depth of maximum dose $\left(\mathrm{d}_{\max }\right)$ using a $6 \mathrm{MV}$ Linac. The experiment was repeated using a glass phantom of the same dimensions to study the effect of diffused oxygen on normalization point. The gels were imaged four days after irradiation and coronal slices (parallel to the beam axis) of 15 and $20 \mathrm{~mm}$ thick were taken through the gel respectively. The dose distribution was then calculated using the method described previously (4.2.1).

\subsubsection{Results}

The comparison of the dose distributions from the $30^{\circ}$ oblique beam produced in BANG gels and Helax-TMS are shown in figures 5.16 and 5.17. It is clear from the 
figure 5.16 that there is a large difference of about $10 \mathrm{~mm}$ in isodose level from 90 to $70 \%$ in the perspex phantom. Once again the exit part of the radiation was not taken into account in measurement as being close to the Perspex wall. Even though the dose distribution in the glass phantom agrees well (within about $2.5 \mathrm{~mm}$ ) with the planning system as shown in figure 5.17 , the gel shows discrepancies of maximum $4 \mathrm{~mm}$ in the $90 \%$ isodose level close to the wall side of the phantom and exit part of the beam.

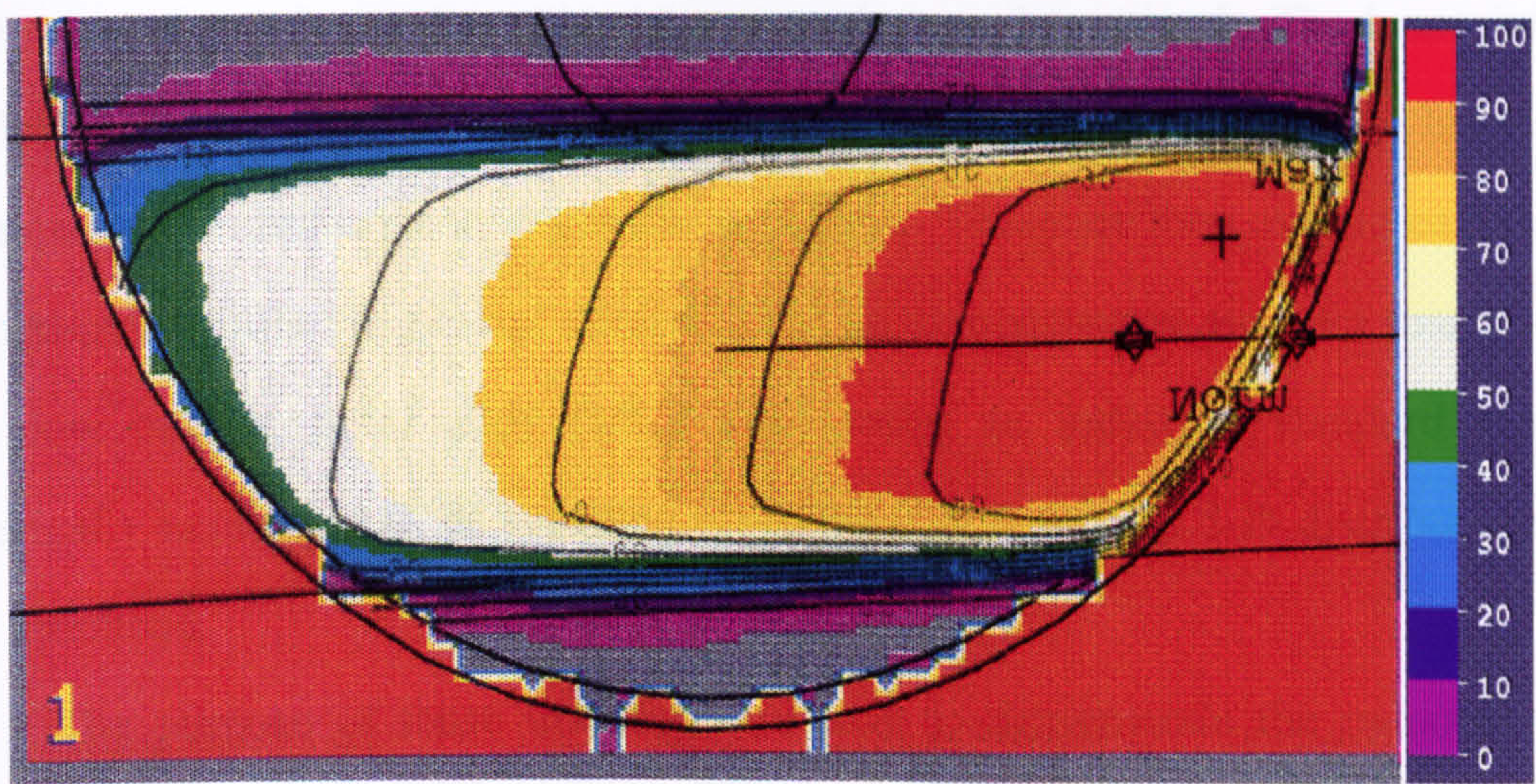

Figure 5.16. Isodose comparison between the gel in Perspex phantom and the plan produced by Helax-TMS

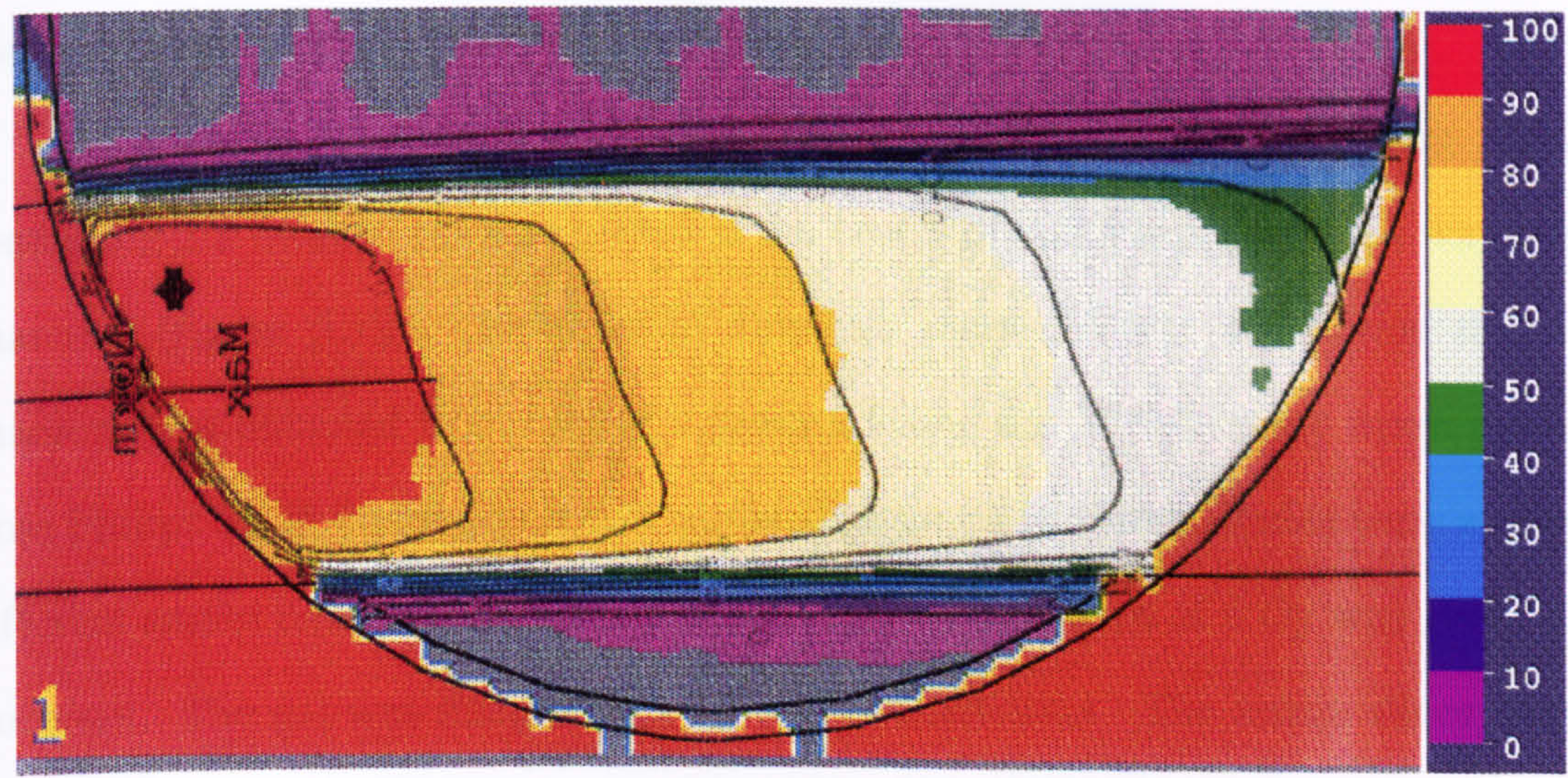

Figure 5.17. Comparison of dose distribution produced in the gel using a glass phantom and the plan produced by Helax-TMS 


\subsection{Irradiation with intensity modulated beams}

One method of achieving conformal therapy is the use of patient specific compensators designed by an inverse planning process. Such a system is being developed at the Walsgrave Hospital, Coventry (Haas 1997). A simplified view of the process is as follows:-

The first stage is to select the required dose prescription. Then using a coplanar beam orientation scheme the optimal beam angles are determined. The beam intensity profiles are then optimised using a hybrid scheme combining an iterative least square technique and a multi-objective genetic algorithm. These are then used to calculate the compensator profile (shape and thickness). The compensators are then manufactured from low melting point alloy (MCP 200) using an industrial standard computer numerically controlled $(\mathrm{CNC})$ milling machine. To date only 2-D distributions have been produced.

The purpose of these investigations was to demonstrate the outcome of the conformal plan and assess the accuracy of the predicted dose distribution using both gel and film. The test case which represents a concave pelvic tumour with two organs at risk (OAR) is shown in figure 5.18. The aim was to treat the tumour to the $90 \%$ level whilst delivering less than $20 \%$ to the organs at risk.

To verify the prescribed distribution using compensators a Perspex phantom containing 2.5 litres of gel was placed at the centre of an anthropomorphic water-filled phantom (figure 5.19). In order to simulate more realistic conditions the water phantom was obtained from cast of a patient. Using the $25 \mathrm{MV}$ x-ray beam produced from Philips SL25 linear accelerator at the Walsgrave Hospital 5 fields were applied at different angles using customised compensators. A total dose of $6 \mathrm{~Gy}$ was given to the isocentre. The gel was imaged four days after irradiation. The image slices were 20 $\mathrm{mm}$ thick and were taken from planes parallel to the beam axis and perpendicular to it throughout the gel. The dose distribution from this experiment was then compared with measurements made by Mills (1996) at the Walsgrave Hospital using radiographic 
film. The film was exposed in the centre of a solid homogeneous hardboard phantom of the same dimensions.

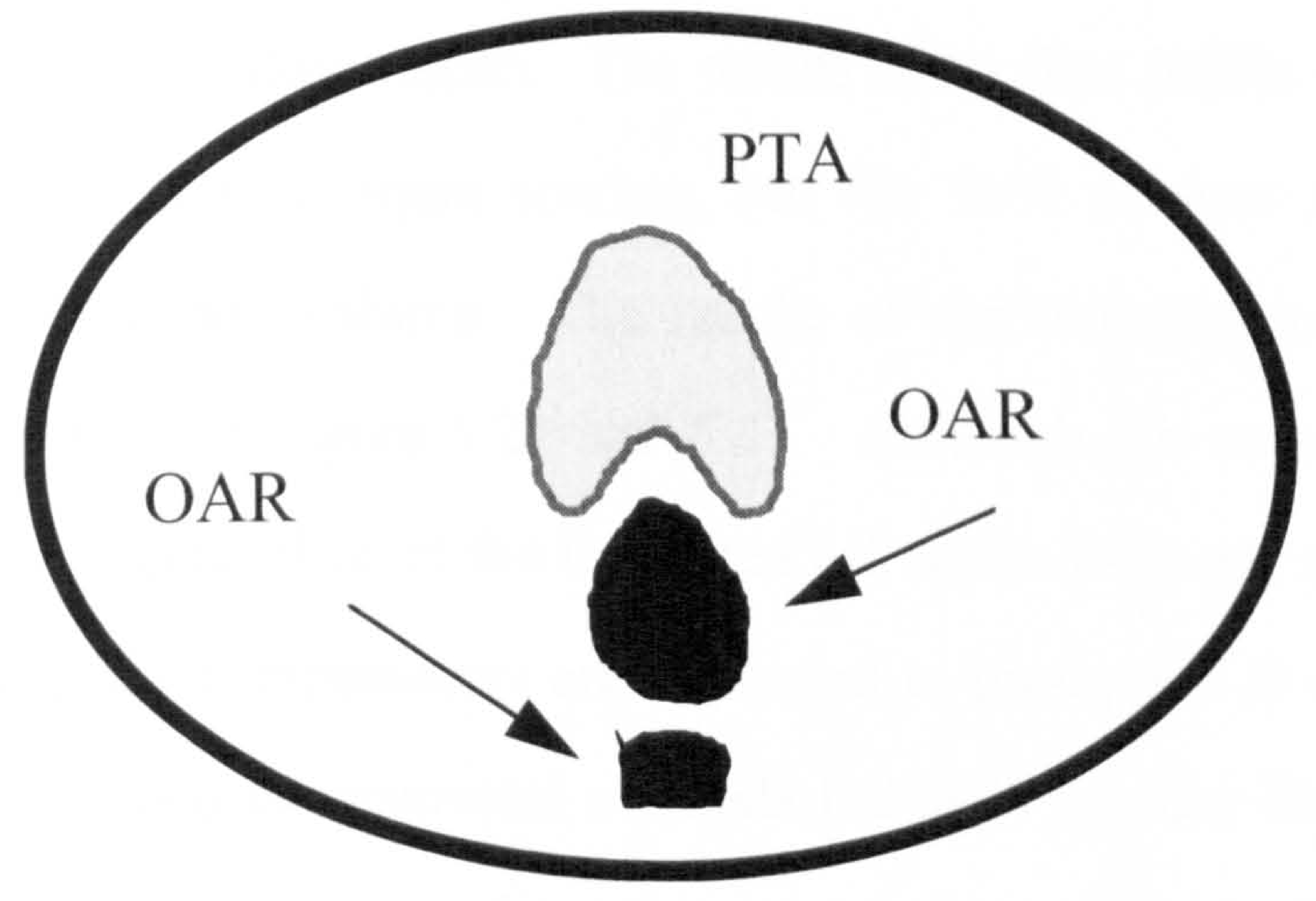

Figure 5.18. Diagram shows the test case with prescribed treatment area (PTA) which is supposed to represent the prostate and two OAR.

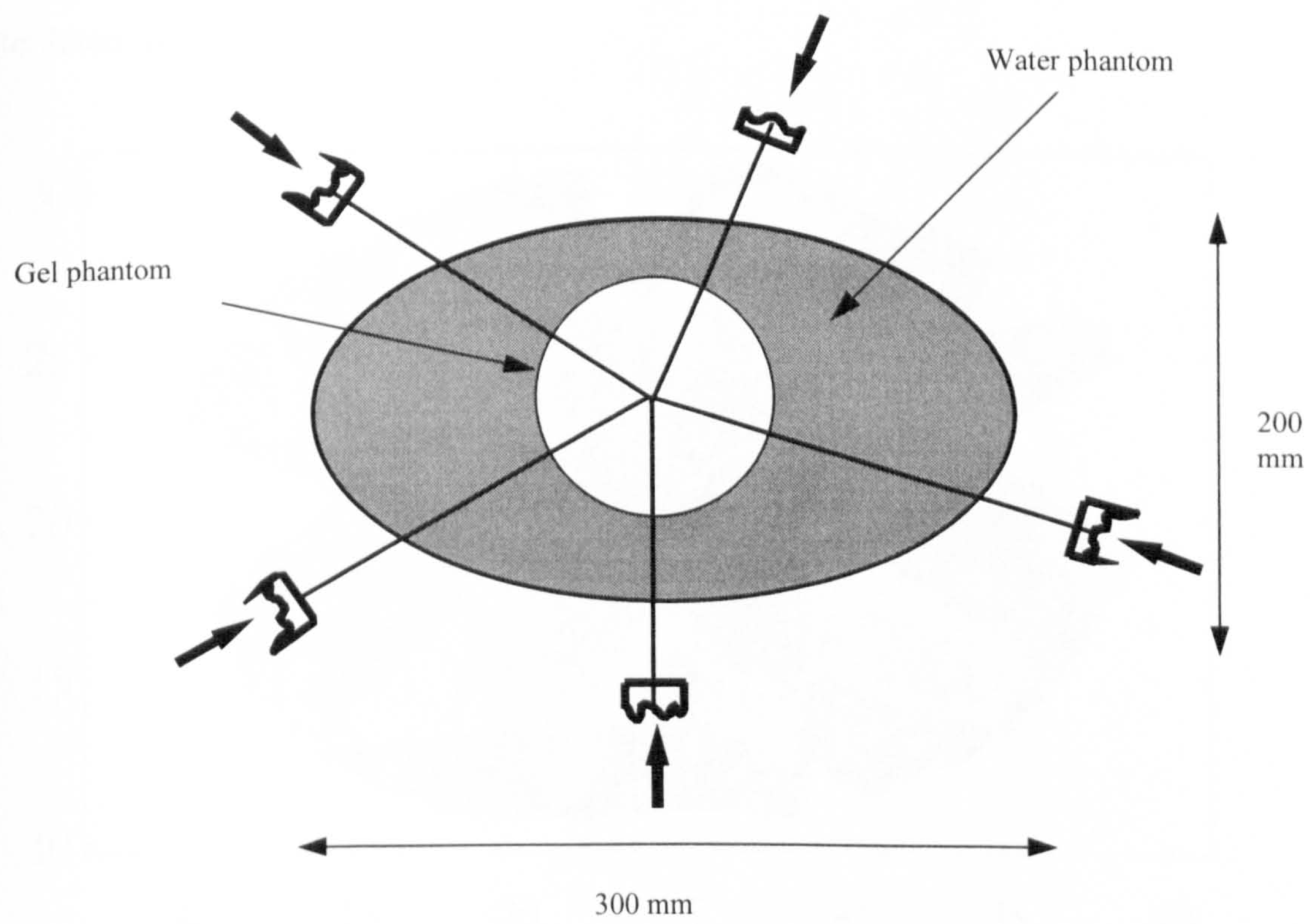

Figure 5.19. Schematic diagram shows the gel inside the anthropomorphic phantom with the 5 field treatment using customised compensators 


\subsubsection{Results}

Figure 5.20 shows the predicted isodose contour. The organs at risk are not shown but are in the lower dose region. The results using film (Mills 1996) are shown in figure 5.21 demonstrating organ sparing, but the $90 \%$ isodose region is not as symmetrical as the planned volume. The results of the measurements with the gel phantom are also shown in figure 5.22 and 5.23. Although this measurement is 2-D figure 5.22 shows a sagittal slice of the irradiated gel which indicates the potential 3-D measurements when the compensators are expanded to produce 3-D distributions. In order to compare the two experimental methods the $90 \%$ isodose line from the film measurement has been superimposed on the gel results together with the calculated $90 \%$ isodose line. It can be seen that there is reasonable agreement between the two methods. Both experiments show the same asymmetry and slight overdose in the top left hand corner and an under dose in the posterior region when compared with the calculated distribution.

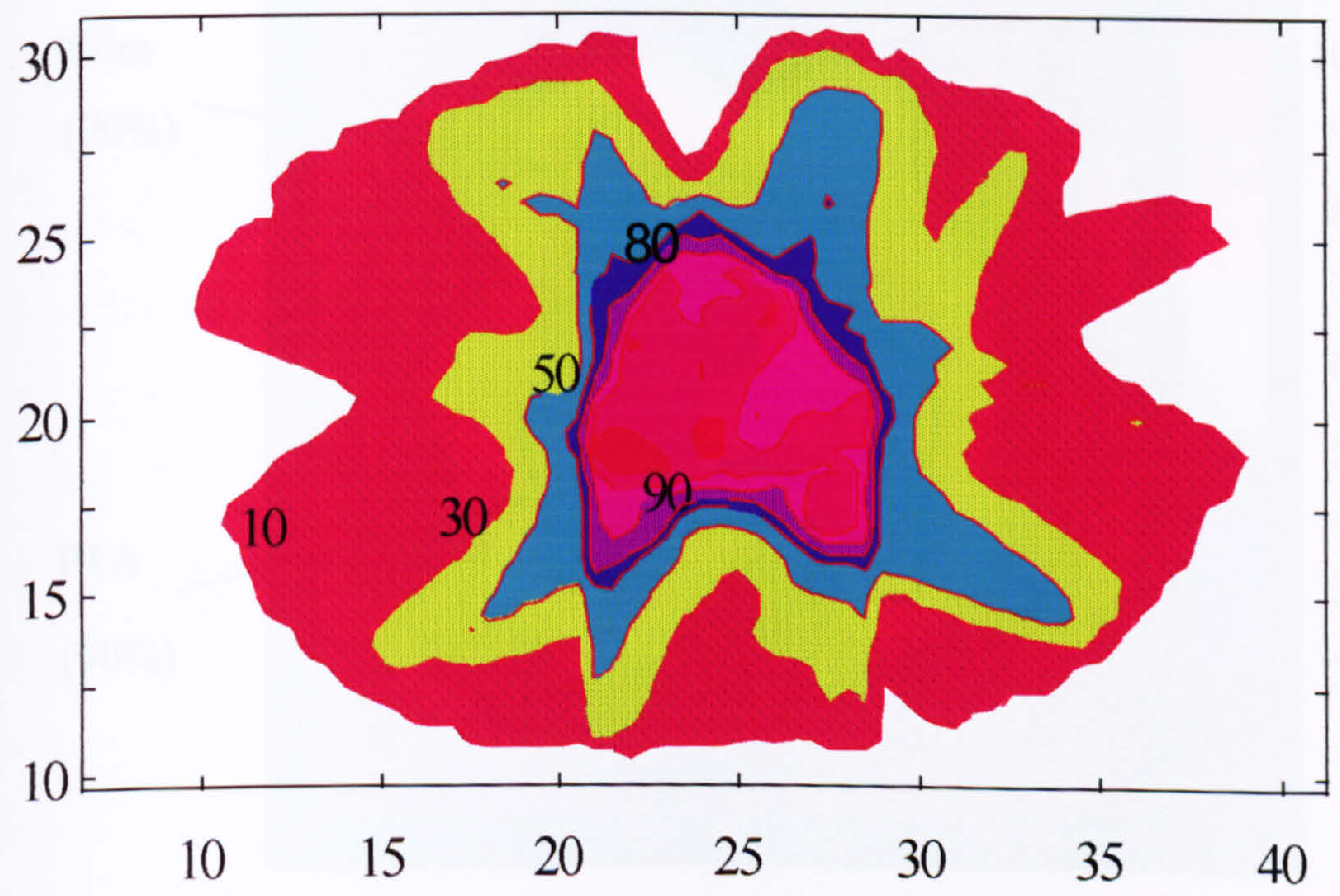

Figure 5.20. predicted dose distribution in aim of sparing organs at risk 


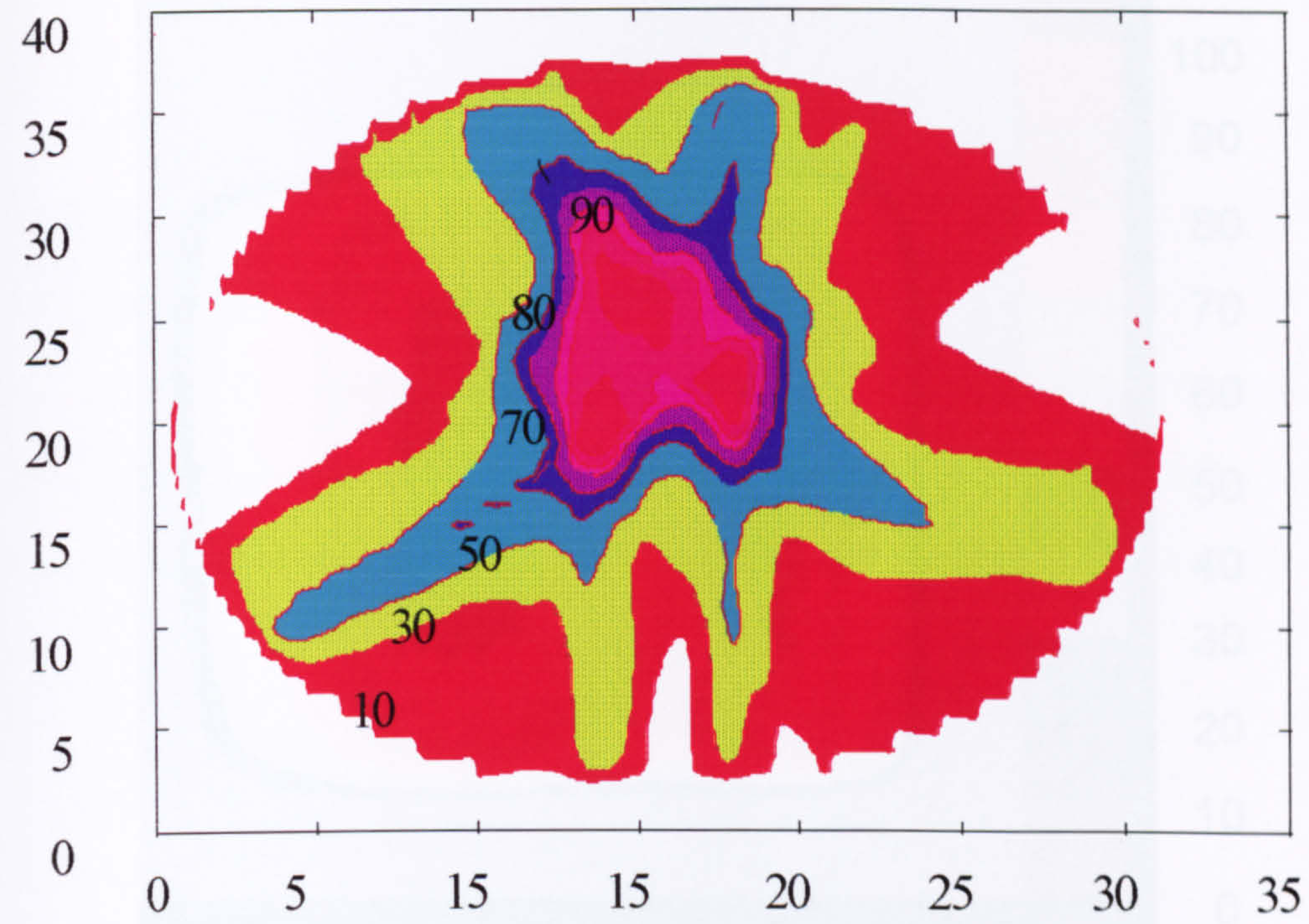

Figure 5.21. Dose distribution obtained from irradiation of the film using conformal treatment scheme.

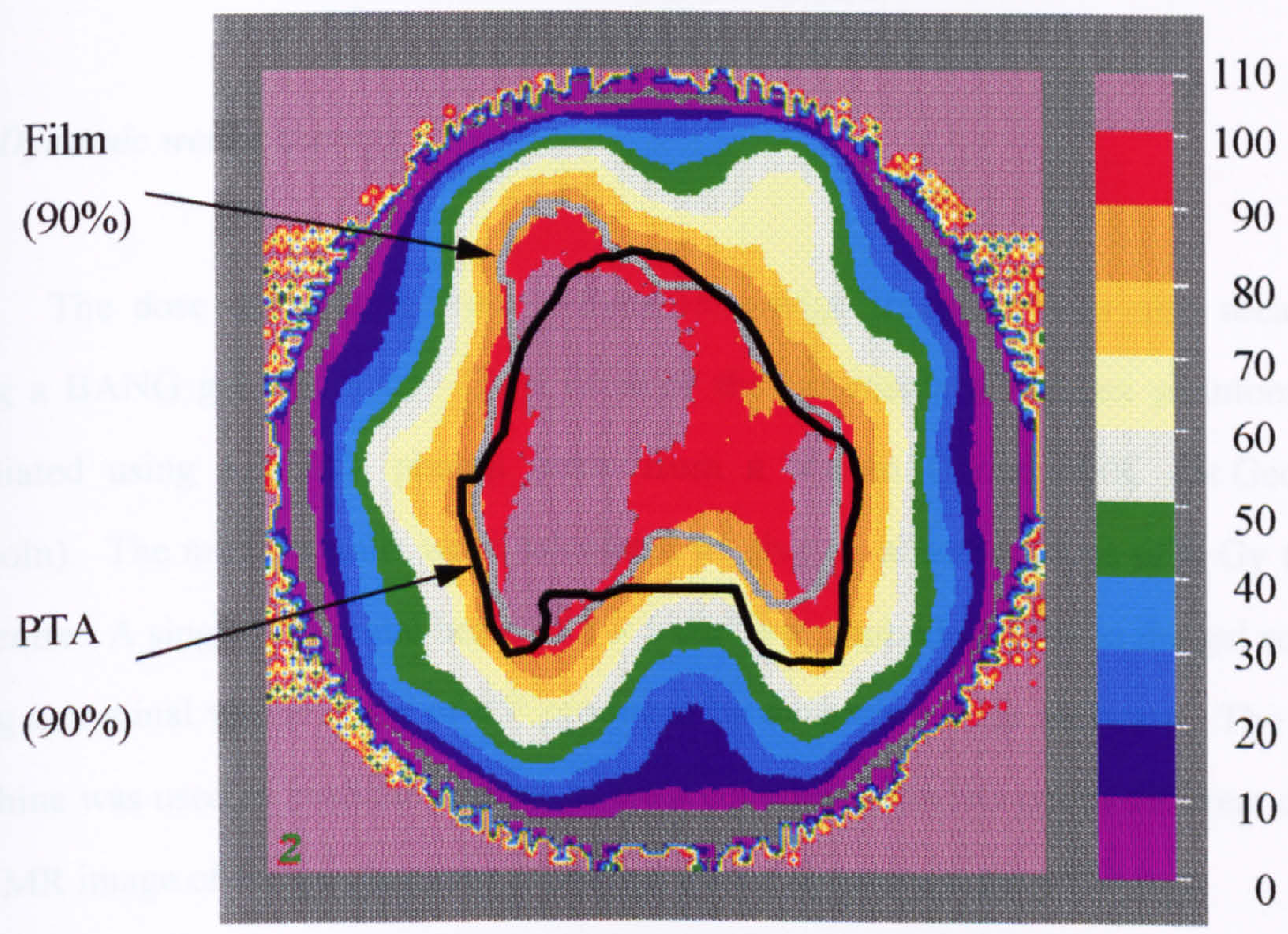

Figure 5.22. Dose distribution produced in the gel by conformal treatment and comparison of the $90 \%$ isodose level in the gel and film with calculation 


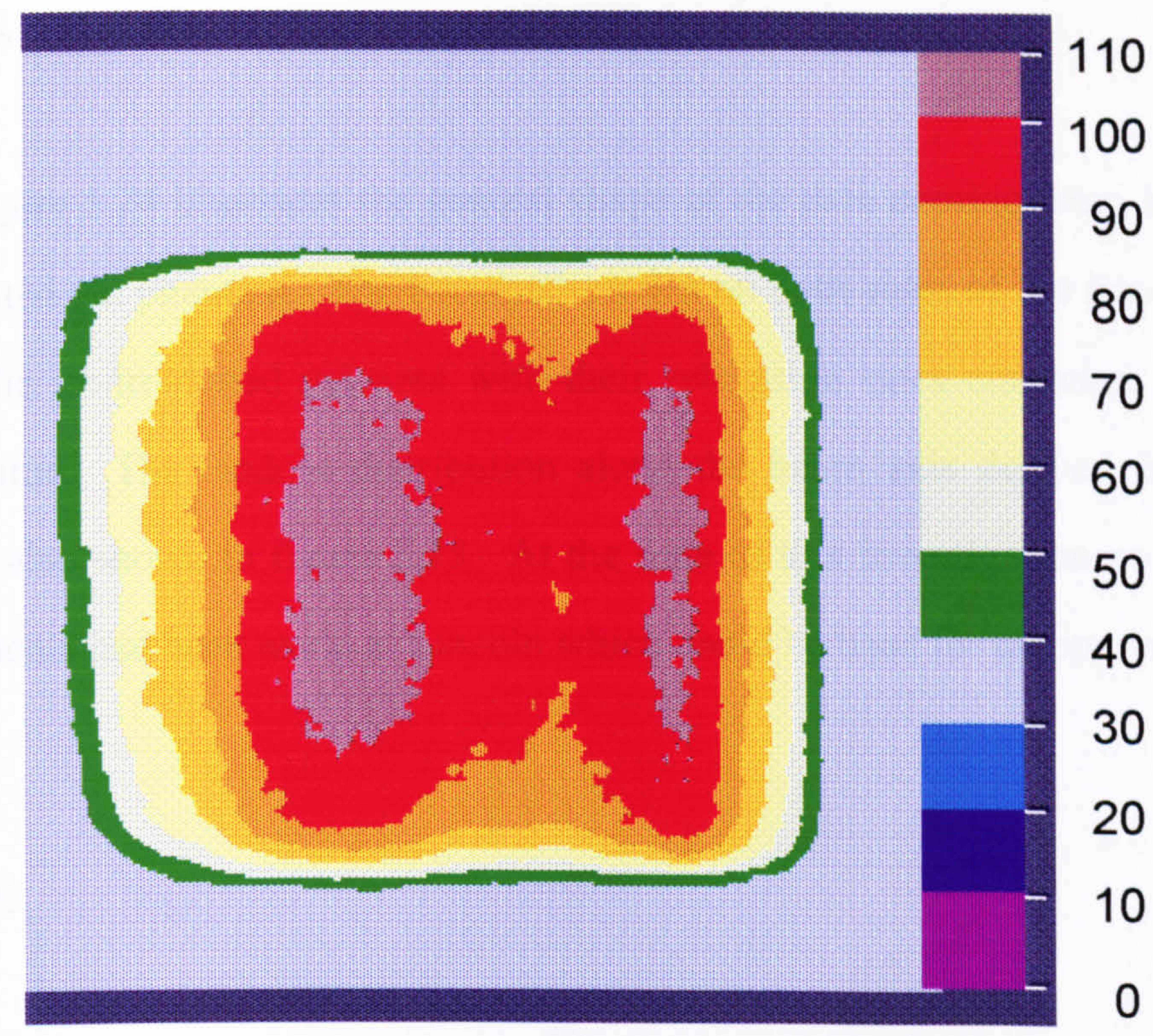

Figure 5.23. Sagittal slice of the gel irradiated using conformal treatment scheme.

\subsection{Dynamic wedge therapy}

The dose distribution from a dynamic wedge treatment was also measured using a BANG gel dosimeter. Two litres of the gel inside a Perspex phantom was irradiated using a $6 \mathrm{MV}$ photon beam from a Varian Clinac 600C (St.Georges, Lincoln). The monitor units were calculated to give an absorbed dose of 6 Gy to the isocentre. A single horizontal beam of $5 \times 5 \mathrm{~cm}^{2}$ was applied parallel to the gel surface using a nominal wedge angle of $45^{\circ}$ produced by dynamic wedge software. The same machine was used to irradiate a series of vials containing BANG gel to different doses. The MR image of the gel then was produced with a slice thickness of $20 \mathrm{~mm}$. 


\subsubsection{Results}

Figure 5.24 illustrates the general shape of the data coming from MR image of the irradiated gel phantom. The two semi-circles on both sides of the irradiated region indicate the un-irradiated gel area with their relaxation rates corrected for the back ground value. The isodose distribution along the beam axis derived from the MR images is also shown in figure 5.25. At the time of this investigation no independent measurements had been made at Lincoln which could be used for comparison.

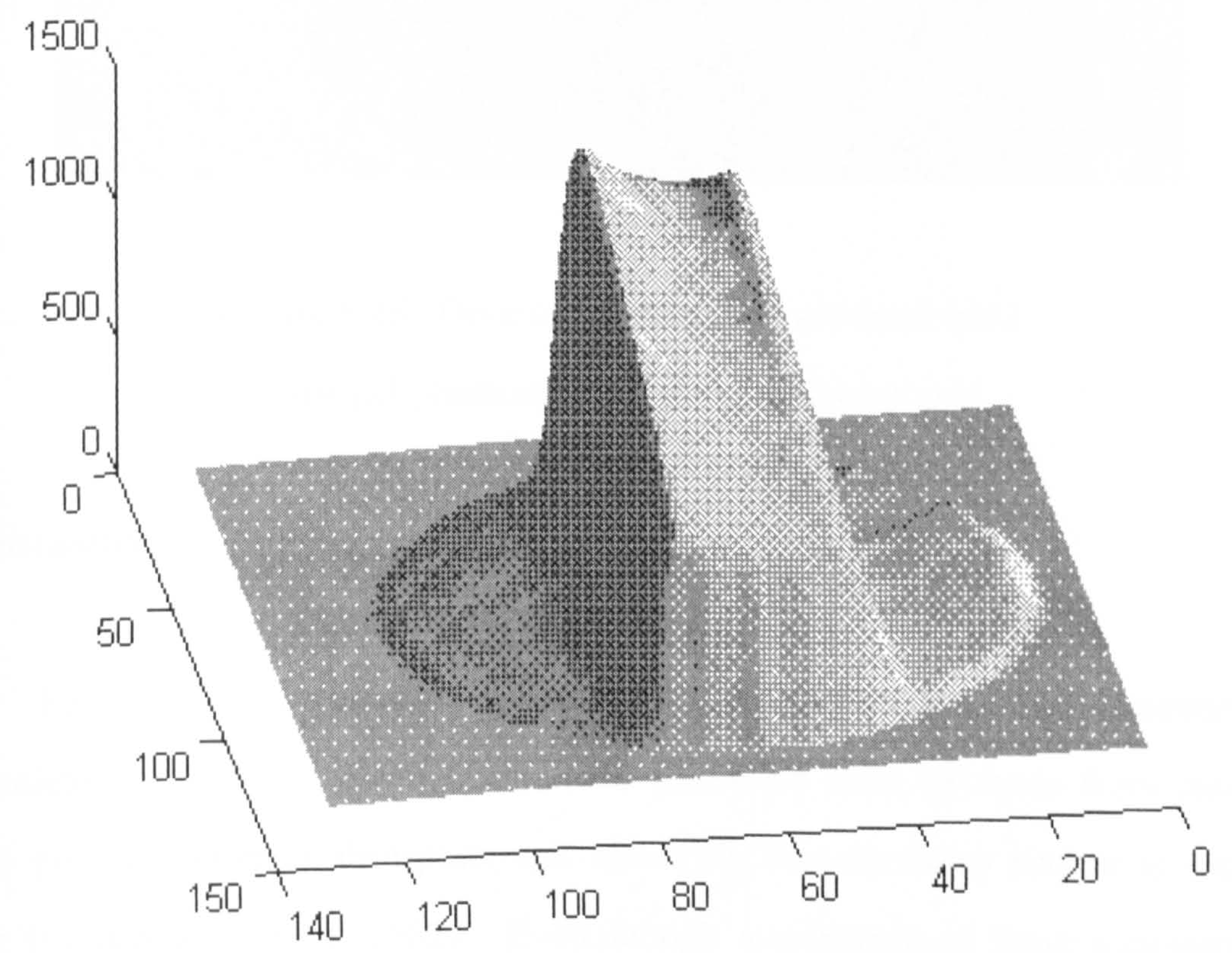

Figure 5.24. A topographic plot of dose distribution derived from the polymer gel irradiated by a dynamic wedge with a nominal angle of $45^{\circ}$ 


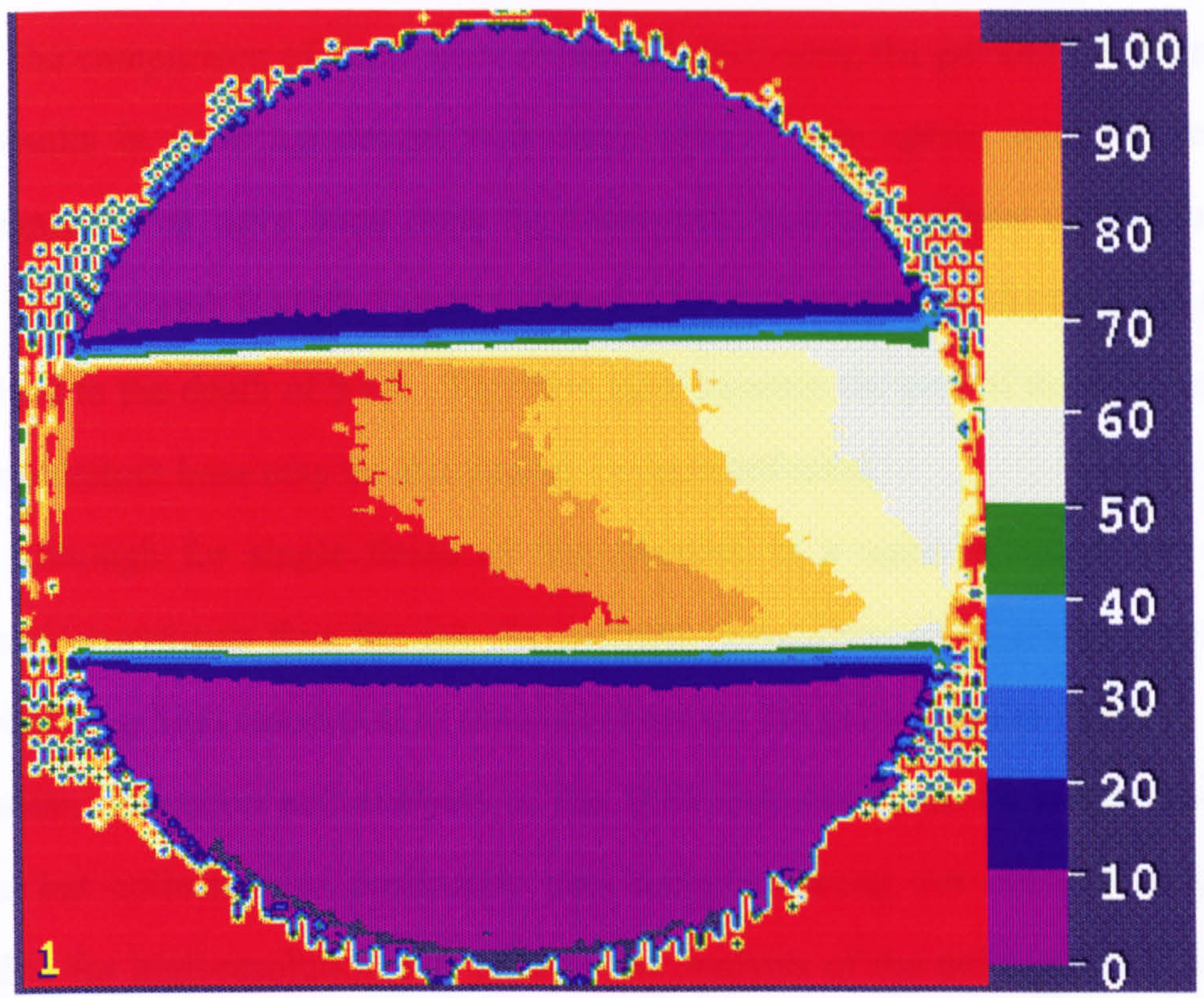

Figure 5.25. Dose distribution derived from MRI

of the gel phantom taken along the beam axis.

\subsection{Discussion}

For dosimetric purposes it is often preferable to use solid phantoms for convenience instead of water. These solid phantoms must be made from materials which provide radiation absorption and scattering characteristics similar to those of water (Constantinou et al 1982). Even though availability of these phantoms can improve and facilitate dosimetric conditions, questions still arise about the perturbation caused by the dosimeter. For BANG polymer gels the dosimeter and phantom are the same and are tissue equivalent. The investigation of the main properties of the BANG gel in chapter 3 shows that the polymer gel dosimeter has the potential to solve most of the current dosimetric problems. The application of polymer gels to practical radiotherapy has demonstrated good agreement with conventional dosimetry and the ability to test treatment planning algorithms. 
The comparison of depth dose measurements using the gel and ion chambers in water results in good agreement with each other. This agrees with the results of measurements that have been done by Maryanski and co-workers using diodes and BANG (1994), and BANG-2 gels (1996a). In this study the depth dose data are normalised to the depth of $5 \mathrm{~cm}$. This was done because the part of the gel close to the Perspex phantom base may be affected by oxygen diffusion.

Although for single fields an ion chamber in a water phantom can provide quicker measurements than the gel, for complex measurements involving two or more fields requiring high resolution in three-dimensions the gel is a faster method. The gel preparation, collection and analysis of the MRI data appear to be a time consuming process, but collection of dosimetric data using scanning ion chamber is probably unfeasible for high-resolution 3-D plans. The analysis of the gel relaxation times and conversion to dose distribution is certainly no more time consuming than the analysis of data from scanning chamber and could be completly automated to provide 3-D dosimetric information within minutes.

The comparison of three and four field treatment plans measured in a Perspex phantom showed a close agreement between the gel and Helax-TMS except for the isodose levels close to the phantom wall. This difference is caused in part by the fact that Helax-TMS does not account for the lack of backscatter (Hejira et al 1998). In addition there are oxygen effects due to the use of Perspex. Plastic containers containing hypoxic media allow diffusion of atmospheric oxygen which passes slowly into the medium. The oxygen either diffuses through the Perspex or is released from the walls. Therefore such differences in the result could be explained by oxygen diffusion through the phantom wall. However using a glass phantom overcomes this problem. Using a glass phantom, the gel and treatment plan shows some disagreement in isodose levels close to the beam exit. This is explained by the fact that Helax-TMS does not account for the lack of backscatter.

In contrast to the three and four field treatment plans, the oblique beam treatment resulted in a disagreement between the Helax-TMS plan and the gel in the 
Perspex phantom. It is believed that the reason for this is the effect of oxygen, which would reduce the sensitivity thus reducing the value of the normalisation point as this is close to the wall of the phantom. The results obtained using a glass phantom support this. The discrepancy in the $90 \%$ isodose level in the case of the glass phantom may also be due to the Helax algorithm. It is possible that it does not take into account the effect of greater glass thicknesses at such a beam incidence or does not correctly account for the glass gel interface. The disagreement between both systems in the region of beam exit can be explained by lack of backscatter which Helax does not account for.

It would appear that Perspex is not a suitable phantom for gel dosimetry. However, if the area near the phantom wall is not of interest and it is the central region that is most important, Perspex can be used. In addition the flexibility of the Perspex to make a phantom of any desired shape and volume is a factor which could facilitate the application of gel dosimetry in radiotherapy. The oxygen diffusion is more pronounced at the edge of the phantom and the central region normally remains unaffected. The comparison of dose distributions in the Perspex and glass phantoms demonstrated that the oxygen effect is not equal in the entrance and exit part of the beam as would be expected. The effect is less in the beam entrance, where the dose is higher, than in the beam exit. This supports the earlier comment (3.6.2) on the inhibiting effect of oxygen on polymerization. However it seems the use of the Perspex as a phantom material should not be totally ruled out. Alternatively other plastics with better diffusion characteristics should be investigated and these could be used as materials for phantom walls.

Introducing an air gap inside the gel gave encouraging results. Although the dose distribution produced in the gel resulted in a reasonably good agreement with the Helax-TMS treatment plan, there was still some disagreement due to the phantom wall and Perspex tubing. In this measurement it was not possible to compare the gel and Helax-TMS to the same extent as other external field measurements because of the effect of the phantom material and the Perspex insert. Further measurements are 
required using a glass phantom and glass insert or oxygen resistant plastic. Additional measurements could also be made to compare the gel with ionisation chamber measurements.

The verification of the absorbed dose distribution in conformal therapy is needed to confirm that complex dose distributions can be delivered in practice. Conventional dosimeters are not suitable for such a measurement due to their various limitations. The results obtained with the gel show good agreement with the dose distribution produced using radiographic film (Hass 1997). Both methods show similar discrepancies in the left anterior oblique and posterior regions compared with the calculation. Possible reasons could be a slight error in the settings of the beams or the positioning of the compensator, or more obviously in the algorithm used in the calculation. It has been reported that a small error of $0.5 \mathrm{~cm}$ in beam offset can result in a large difference in the dose distribution (Hass 1997). However it has been proved that polymer gel can be used to verify a more complex treatment plan. Similar conclusions have been drawn by Baustert et al (1997).

So far the experimental conformal therapy at Walsgrave Hospital has been restricted to two dimensions, but work is now in progress to establish full three dimensional conformal therapy using customized compensators. Therefore the three dimensional nature of the gel, will make it a unique candidate for measurements.

One of the major concerns in the dosimetry of dynamic wedges is measurement of the dose distribution. Since width of the field and also dose rate varies during the process it needs a dosimeter that will integrate each measurement point within the phantom during the entire dose delivery (Leavitt and Larsson 1993). The reproducibility of the film measurements is difficult due to both variation in the calibration at different depths and processing conditions (Bidmead et al 1995 and Elder et al 1995). Using a diode array to produce isodose lines is time consuming as it needs a large number of measurements and also the size of the diode limits the spatial resolution of the measurements. The BANG polymer gel offers several important advantages in measurement of dynamic wedge. The in-plane spatial resolution of the 
gel in this measurement is $0.75 \mathrm{~mm}$ which is about three times less than the width of the diode detector and it can be enhanced by decreasing the field of view. It is also a more flexible method, as the beam data is measured in 3-D in the gel using only minutes of the machine time. The beam profiles and percentage depth doses can then be obtained at any convenient time after irradiation. It can be concluded from the measurements that polymer gel can be an alternative method to verify dose distribution in dynamic wedge beam.

The gel proved to be quite stable during transport to and from different sites. For example some of the measurements in this study were carried out in quite long distances from where the gel was manufactured, often in excess of 50 miles. To date no problem was found with the gel during travelling to the centre where it was irradiated. Therefore it is believed that the polymer gel dosimeter can be also used as a dosimeter for inter-comparison purposes. The experience of Ibbott et al (1995) in mailing the BANG-2 for QA purposes and also commercialising the gel and sending to different centres i.e. from USA to London (Maryanski 1997) confirms this statement. 


\section{Chapter 6. Other applications of the BANG polymer gel}

\subsection{Boron neutron capture therapy (BNCT)}

\subsubsection{General background}

Boron neutron capture therapy (BNCT) is a specialised treatment for malignant disease in the brain. The technique is based on the capture of thermal neutrons by boron-10 $\left({ }^{10} \mathrm{~B}\right)$, which has previously accumulated in the tumour cells. The method takes advantage of the large thermal neutron capture cross section for boron-10 and of the subsequent energy released in the region of the tumour cells. Irradiation of a tumour containing ${ }^{10} \mathrm{~B}$ with thermal neutrons produces an alpha particle and a ${ }^{7} \mathrm{Li}$ ion from the reaction ${ }^{10} \mathrm{~B}(\mathrm{n}, \alpha){ }^{7} \mathrm{Li}$ together with a high energy gamma ray. The high linear energy transfer (LET) and short range of ${ }^{7} \mathrm{Li}$ and the alpha particles, $10 \mu \mathrm{m}$, allows lethal doses to be deposited in the tumour cells. If the concentration ratio of the ${ }^{10} \mathrm{~B}$ between tumour and normal cell is large, the healthy tissue is relatively undamaged (Perks et al 1988, Pöller et al 1992, Allen and Beynon 1995). Therefore treatment of tumours by BNCT requires both a suitable pharmaceutical or carrier to deliver ${ }^{10} \mathrm{~B}$ into the tumour and obviously a suitable neutron beam.

In order to deliver ${ }^{10} \mathrm{~B}$ preferentially to cancerous cells it must be bound to an appropriate carrier and administrated by oral ingestion or intravenous injection. There is a significant decrease in blood-brain barrier in the region of a tumour which will help this process. It was reported that the past failure of BNCT clinical trials in the USA was due to the carrier of the boron (Gabel 1996). However the recent development of new boron carriers such as ${ }^{10} \mathrm{~B}$-L-borophenylalanine (BPA) has lead to an increased uptake of boronated compounds into the tumour (Pignol et al 1998). This increase in the concentration ratio of ${ }^{10} \mathrm{~B}$ in the tumour consequently improves 
the quality of the treatment by increasing the tumour dose while protecting the normal tissue.

In order to evaluate the effects of therapy, a knowledge of the radiation absorbed dose distribution in three-dimensions is necessary before treatment. This is because the different contributions to absorbed dose will have different spatial distributions. In addition to the absorbed dose arising from heavy recoil particles from boron neutron capture ( $\alpha$ and ${ }^{7} \mathrm{Li}$ ), there are also gamma rays from hydrogen capture ${ }^{1} \mathrm{H}(\mathrm{n}, \gamma){ }^{2} \mathrm{H}_{1}$, protons from neutron capture by nitrogen ${ }^{14} \mathrm{~N}(\mathrm{n}, \mathrm{p}){ }^{14} \mathrm{C}$, together with absorbed dose due to the gamma component of the therapy beam produced in the target and shielding materials. Theoretical calculations of three-dimensional dose distribution for BNCT is complex and requires sophisticated analytical methods (Nigg et al 1991, Cantone et al 1992, Gupta et al 1993) although a recent paper indicates a simple planning system can give useful results (Raaijmakers 1998).

In this preliminary study, carried out in collaboration with Birmingham University, the polymer gel dosimetry system was used to see if it was possible to identify any dose enhancement due to presence of boron in the BANG gel. The results were then compared with calculations using a Monte Carlo (MCNP) simulation carried out by Tattam (1998).

\subsubsection{An accelerator based epithermal neutron beam}

It is currently accepted that an epithermal neutron beam is preferable to a thermal neutron beam for applications in therapy due to its greater penetration (Allen and Beynon 1995). An epithermal beam will generate a peak of thermal neutrons at a depth of a few centimetres inside the tissue whilst a thermal neutron beam would produce a peak at the surface. Therefore, using epithermal neutron beams for BNCT results in less damage to healthy tissue (Gupta et al 1993). An accelerator-based neutron source is employed at Birmingham to produce an epithermal neutron beam for BNCT. It has been reported that one of the main advantages of the accelerator based 
neutron beam over reactor sources for BNCT is a better neutron energy spectrum with lower gamma ray contamination. It is also less expensive and easier to accommodate in or near a hospital for clinical purposes (Allen \& Beynon 1995). The major components of the accelerator-based facility are a source of protons, a target for the production of the neutron beam and a moderator assembly. The accelerator used at Birmingham is a Dynamitron, a Cockcroft-Walton accelerator. The facility has been described in detail by Allen and Beynon (1995).

\subsubsection{Dosimeter considerations}

In BNCT the absorbed dose to tissue from the complete radiation field is due to neutron, gamma and boron capture components as discussed earlier. This means that total absorbed dose given to tissue or tumour is a combination of the contributions from these components. This makes radiation dosimetry and treatment planning more complex for BNCT than conventional photon therapy. Any successful clinical application of the technique will rely on accurate calculations or measurements of the dose distribution resulting from these interactions.

\subsubsection{Method and material}

The process of the gel preparation was the same as described in 4.6.1. Before using the gel for BNCT an investigation was made of the effect of adding boron compounds to the gel on either the polymerization process or gel response. Two samples of the gel were prepared using chemicals from the same batches. One was loaded with boric acid ( $30 \mathrm{ppm}$ boron-10). Seven vials were filled for each sample of the gel. In order to measure differences in response, both sets were irradiated with doses ranging from 1 to $9 \mathrm{~Gy}$. For the neutron irradiation two samples of gel were prepared with volumes of 500 and $200 \mathrm{ml}$ respectively. For the purpose of identifying any measure of dose enhancement due to the boron compared with the pure gel the 
larger gel volume was doped with $60 \mu \mathrm{gm}{ }^{10} \mathrm{~B}$ per gm of the gel. $17.5 \%$ by weight of the boric acid is boron. Of this, the abundance of ${ }^{10} \mathrm{~B}$ is $20 \%$ and ${ }^{11} \mathrm{~B} 80 \%$, therefore $0.9 \mathrm{gm}$ of boric acid was used to provide $60 \mu \mathrm{gm}$ of ${ }^{10} \mathrm{~B}$ in $500 \mathrm{ml}$ of the gel. The gel samples were calibrated using a $6 \mathrm{MV}$ x-ray beam. Finally, four vials of $100 \mathrm{~mm}$ height and $24 \mathrm{~mm}$ diameter from each gel were irradiated by the neutron beam. The vials were imaged four days after irradiation and an axial slice of thickess $20 \mathrm{~mm}$ parallel to the beam axis was taken through the middle of the gel.

The gel was irradiated using the Dynamitron at the Birmingham Radiation Centre. A lithium target was bombarded with a $2.8 \mathrm{MeV}$ proton beam. This resulted in the production of a $1.1 \mathrm{MeV}$ neutron beam and also gamma rays with energies of 431 and $478 \mathrm{keV}$ from the target itself (Allen and Beynon 1995). To reduce the unwanted photons produced in the target a lead filter of $2 \mathrm{~cm}$ thick was placed directly in front of the target to shield the gel. The neutrons emerging from the target were then moderated by a water phantom to enhance the thermal neutron component of the beam. The water phantom used was a $14 \times 15 \times 19 \mathrm{~cm}$ Perspex container, and the gel vials were placed in two rows in the water at different depths along the beam axis. A diagram of the experiment is shown in figure 6.1. This geometry was also simulated in the MCNP Monte-Carlo dose calculations. A total radiation time of about $5 \mathrm{hrs}$ was used to give a nominal total dose of $10 \mathrm{~Gy}$ to the gel.

The relaxation time measurements were made using a $1 \mathrm{~T}$ Siemens Impact MRI scanner. The matrix size was reduced to $128 \times 128$ to give a good signal to noise ratio. All other imaging parameters were the same as those described in chapter four (4.2.1).

\subsubsection{Results and discussion}

The dose response curve for boron loaded gel and pure gel is shown in figure 6.2. As can be seen, the transverse relaxation rates for different doses are almost the same in both gels within experimental error of $\pm 4 \%$. Therefore adding boron or a 
boron compound does not seem to affect the response of the polymer gel. A similar calibration curve was measured using 60 ppm boron- 10 .

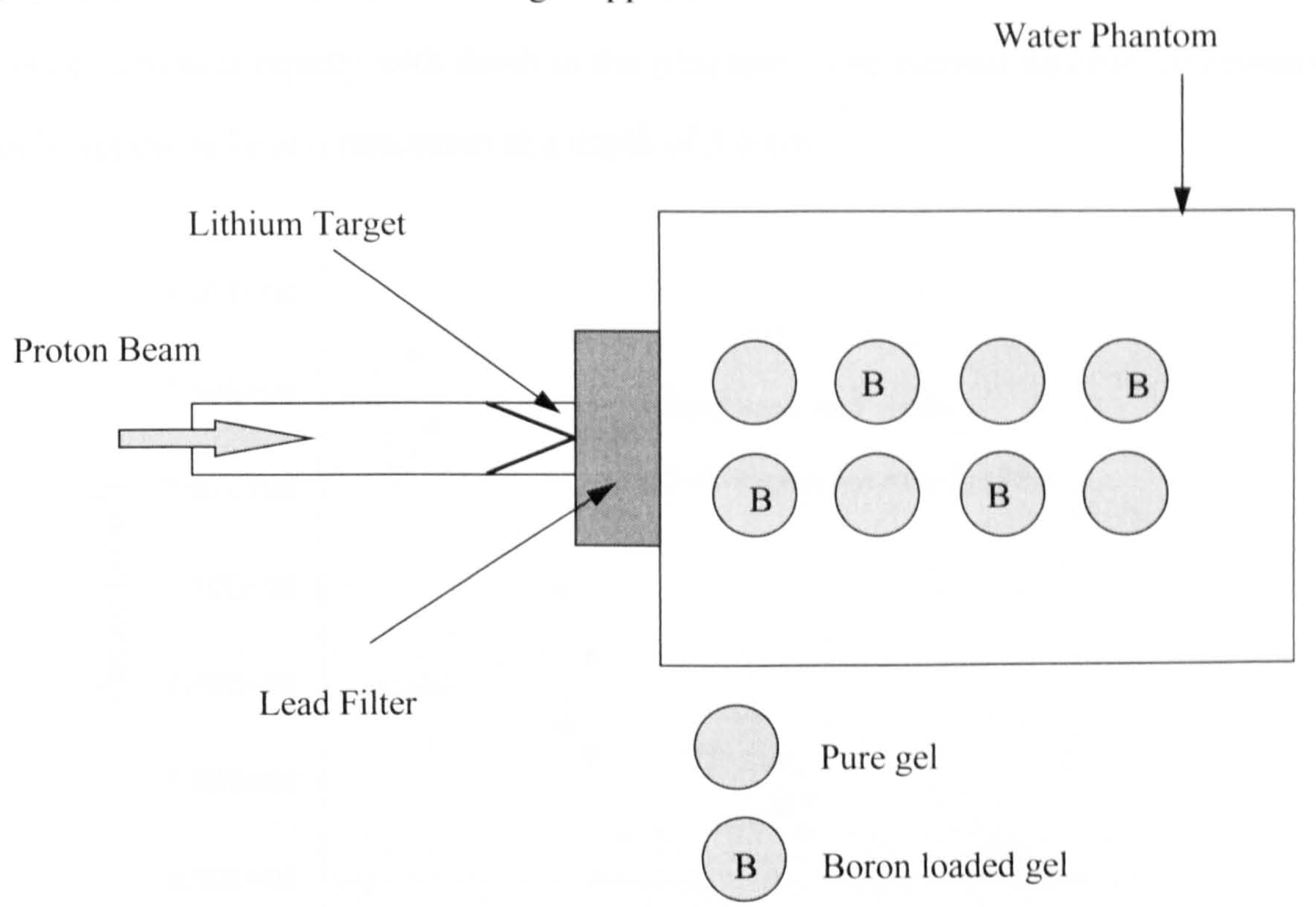

Figure 6.1. Experimental set-up

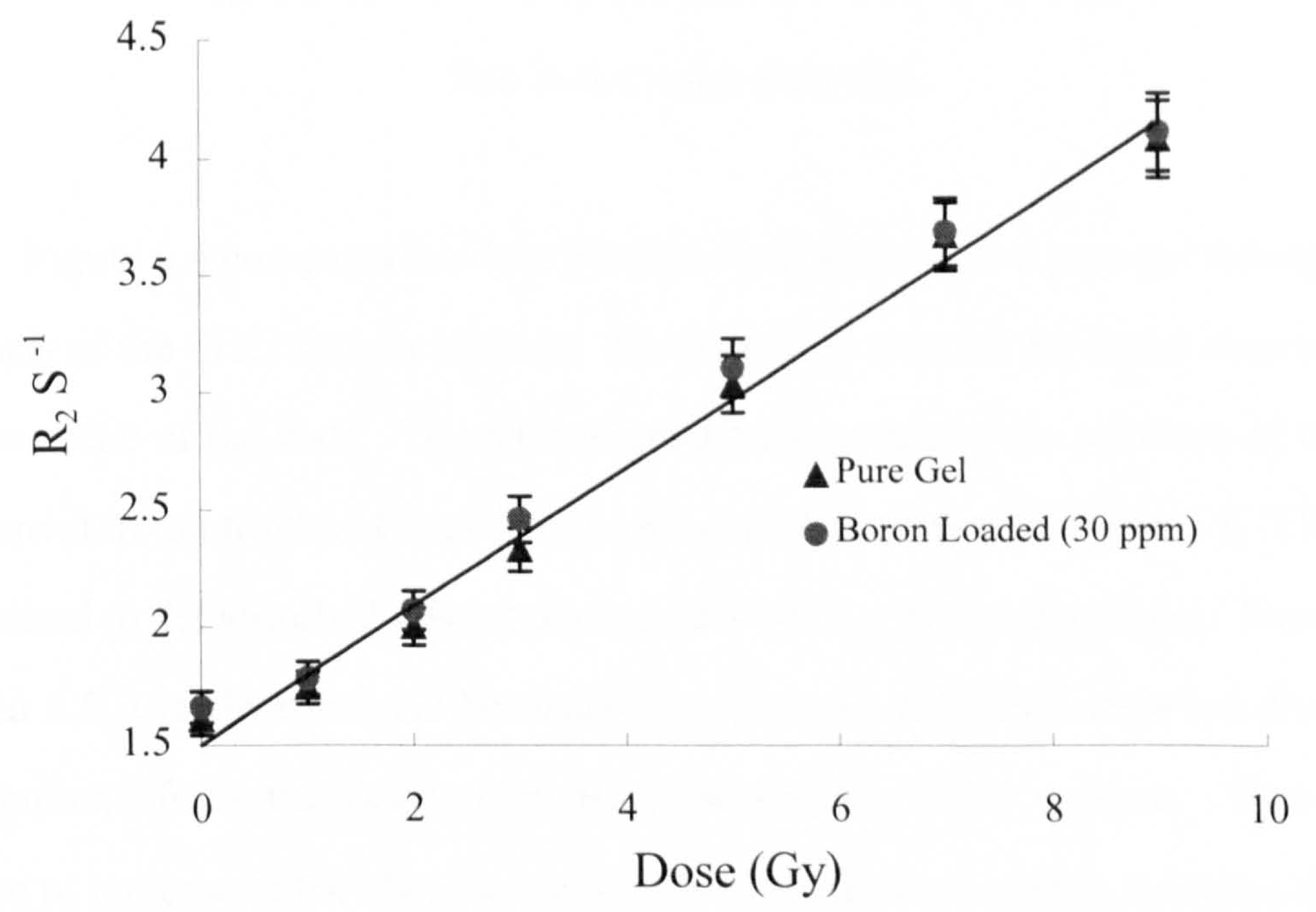

Figure 6.2. The dose response of pure and boronated gel to $6 \mathrm{MV}$ x-ray beam 
The MCNP calculation of neutron fluence in the front and back halves of the vials inside the water phantom is shown in figure 6.3 (Tattam 1998). The total neutron fluence decreases rapidly with depth in the phantom. The thermal neutron component would appear to be at a maximum at a depth of $3.6 \mathrm{~cm}$.

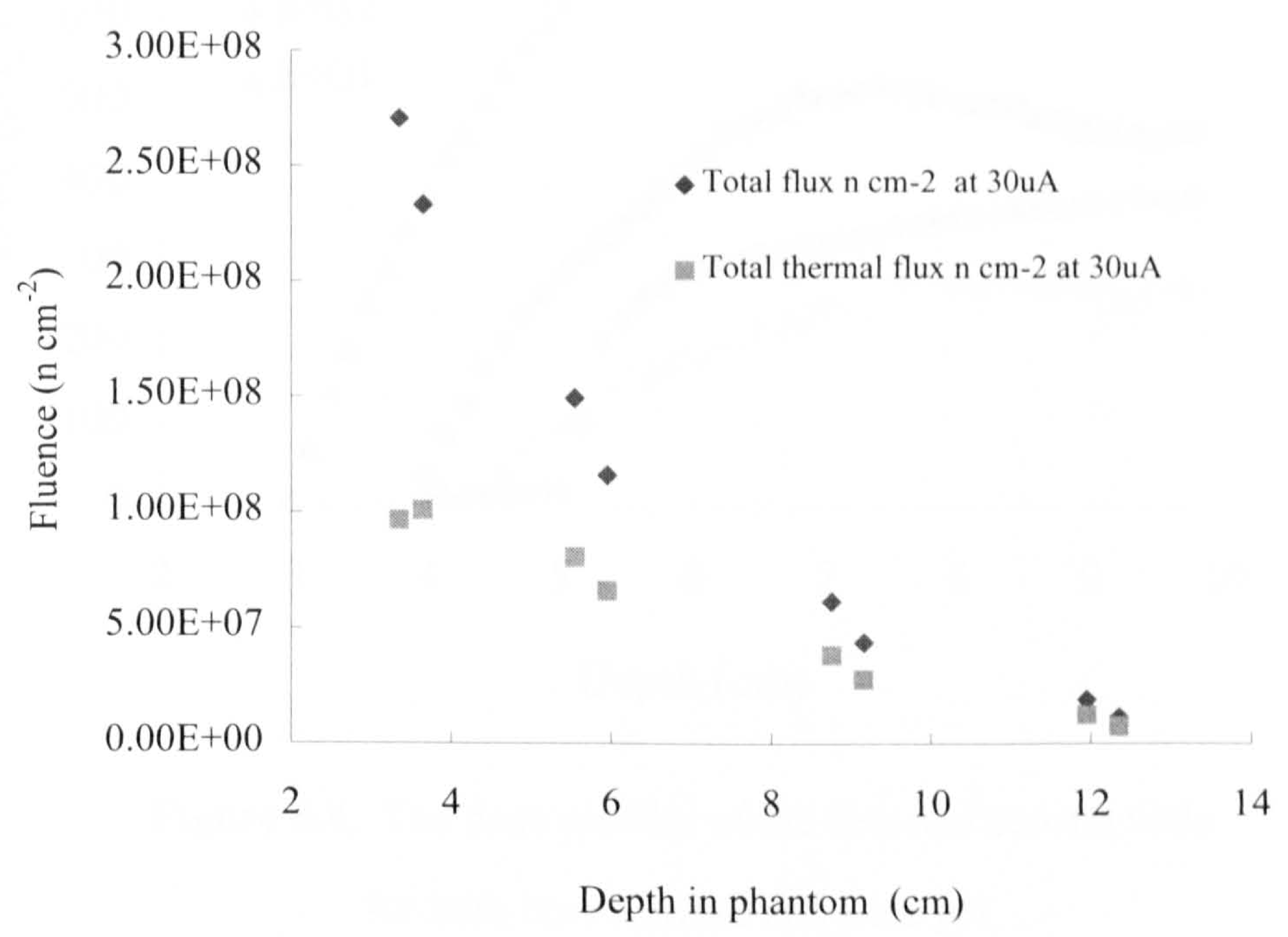

Figure 6.3. MCNP calculation of variation of neutron flux in the water phantom

Figure 6.4 compares the dose profiles for boronated and pure gel throughout the long axis of the first two sets of vials. The high dose area on the figure corresponds to the lower end of the vials. The ratio of enhancement due to the presence of the boron component in the front and back halves of the vials is shown in figure 6.5. The data is normalised to the absorbed dose at depth of $2.4 \mathrm{~cm}$ in a boron loaded gel. From figures 6.4 and 6.5 it is clear that the boronated gel gives an enhanced absorbed dose due to contributions from the heavy particles produced by boron capture. However the amount of increase in the absorbed dose in the second set of vials is less than that in the other vials. The comparison of the calculated dose using the MCNP simulation and the measured dose in the gel with and without boron is shown in figure 6.6. From the 
results it can be seen that there is a reasonably good agreement between the gel and calculation.

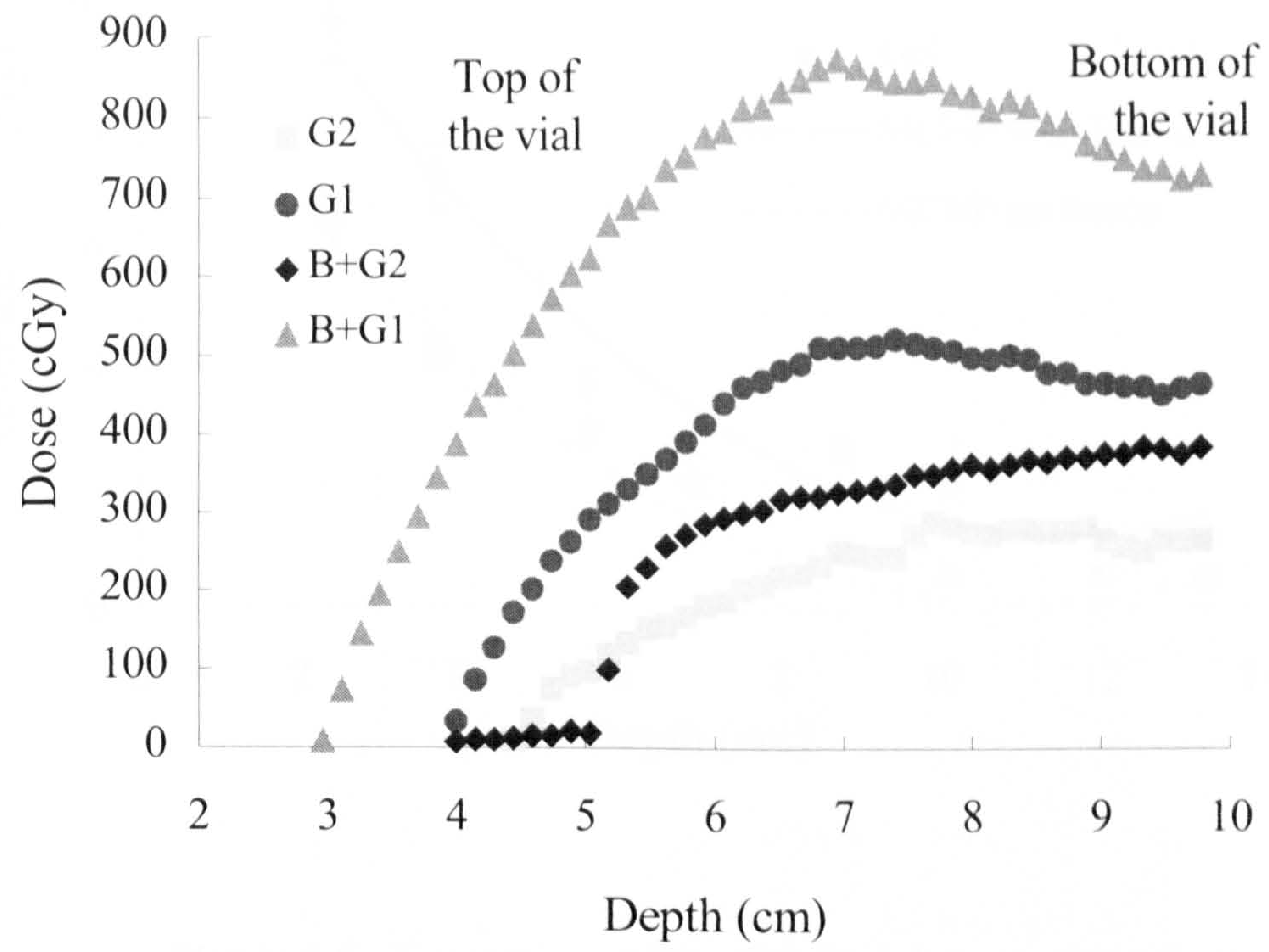

Figure 6.4. The dose profiles of the first and second vials for both boron mixed and pure gel.

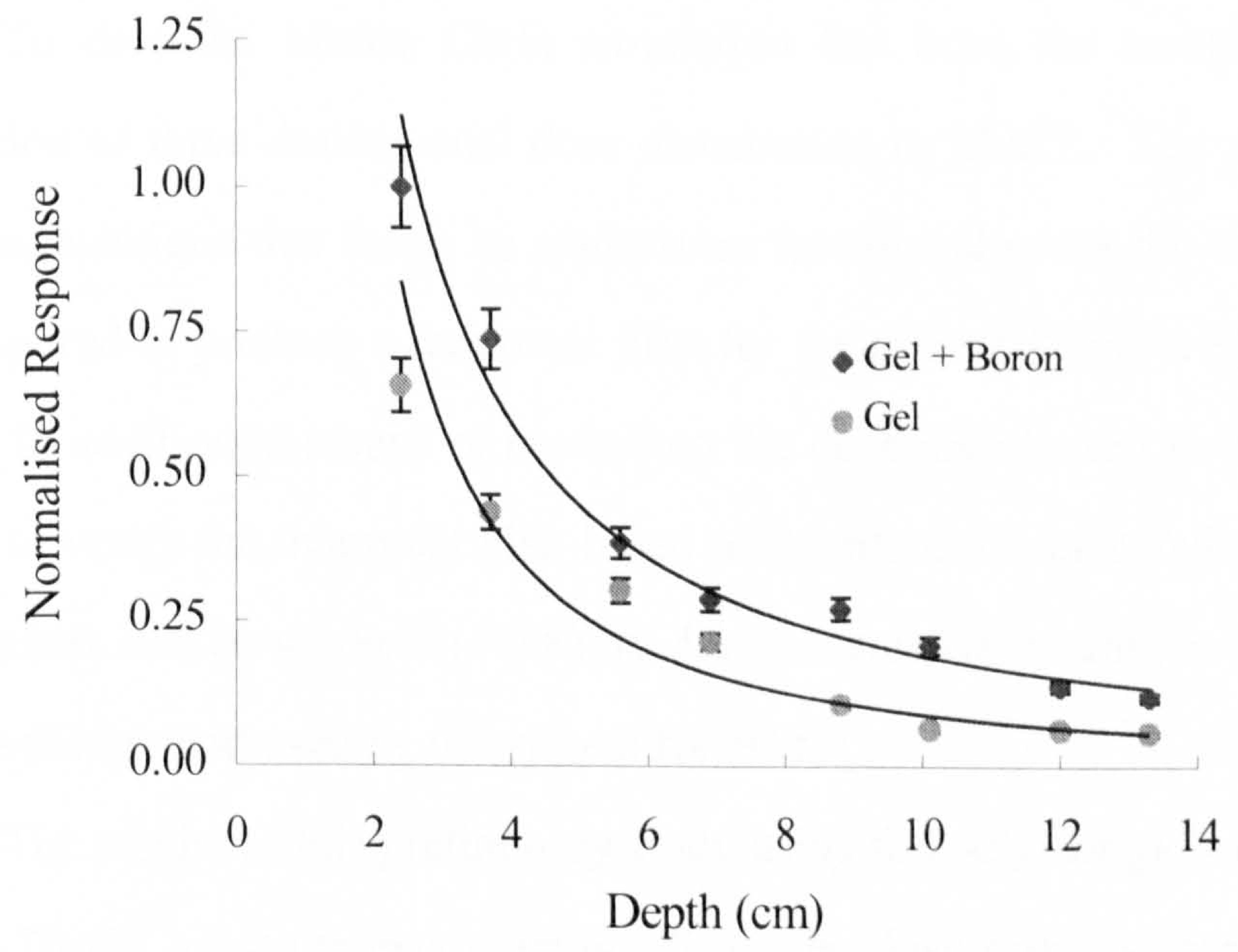

Figure 6.5. Relative doses obtained at different depths from the vials filled with pure and boron loaded gel. 


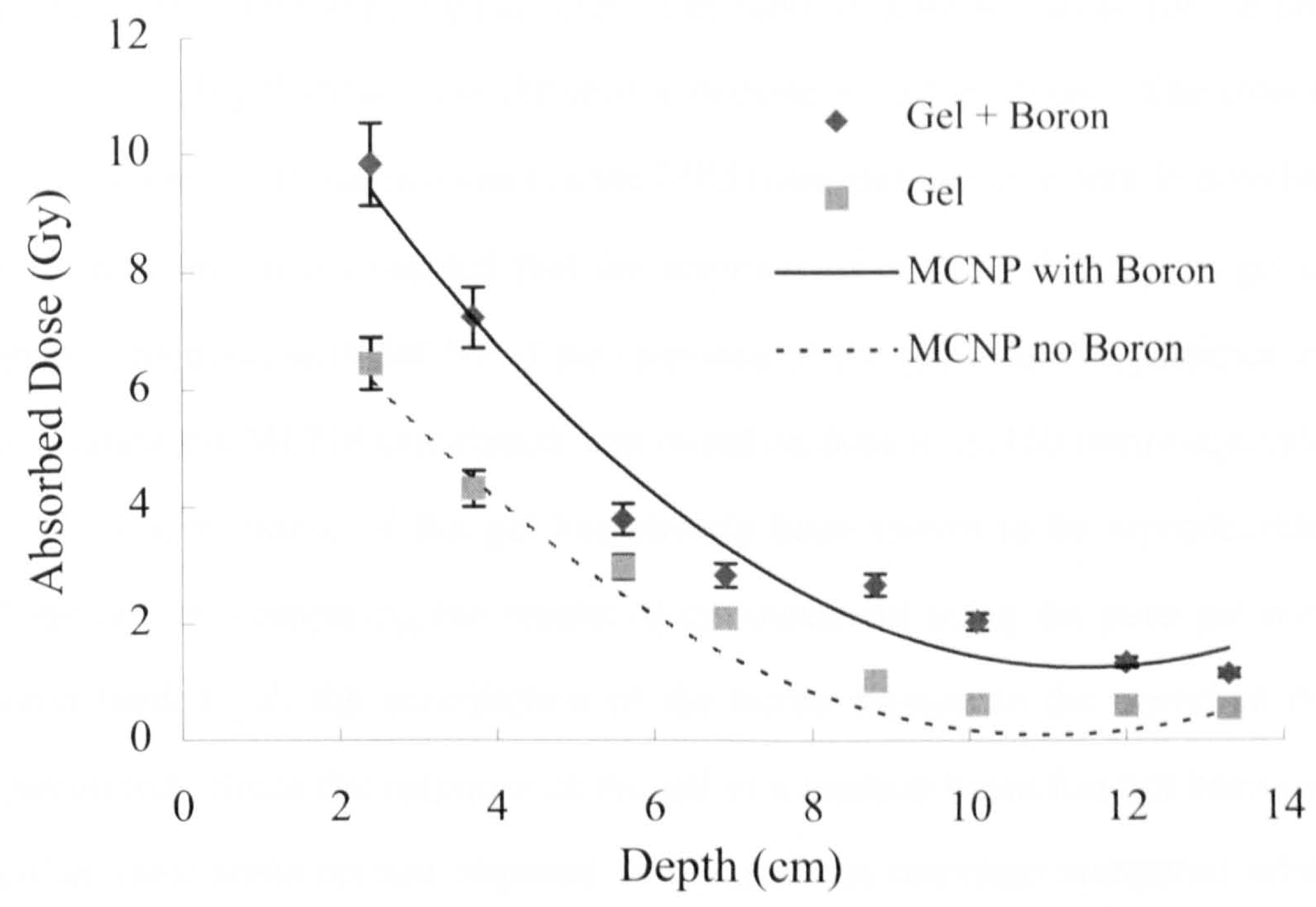

Figure 6.6. Comparison of the absorbed dose resulting from MCNP calculation with dose measured in pure and boron loaded gel.

To date the Monte Carlo simulation has been the technique proposed for calculation of three-dimensional dose distribution in BNCT. The problem associated with this technique that limits its application for clinical use is the amount of computer time required to produce a treatment plan for the patient (Nigg et al 1991, Gupta et al 1993). In addition, a means of measuring the dose distribution in three dimensions is needed to verify the treatment plan based on Monte Carlo calculations. More recently Raaijmakers and co-workers (1998) used a conventional treatment planning system to develop the method used by the system for BNCT.

The results of this preliminary study using the polymer gel to measure absorbed dose in BNCT are quite encouraging. From the dose response curves it appears that mixing boron with the gel does not alter the gel response. The boron loaded gel recorded a minimum of $50 \%$ more absorbed dose than the pure gel due to the 
contribution from the heavy particles. The ratio of absorbed doses for the second set of vials for each gel shows less difference compared to the others. The reason could be misplacement of those two vials in the MRI measurement or errors in positioning in the neutron beam. It is expected that the response of boronated and pure gel could be in better agreement with MCNP if the response of the gel to heavy particles was known. In addition the MCNP calculation was based on dose to A-150 tissue equivalent plastic.

The response of the gel has already been shown to be reproducible (3.7.1.1). Therefore, by comparing the results of measurement using the pure gel with that of a boron loaded gel, the contribution of the boron capture to the absorbed dose can be determined. Since the response of the gel to a neutron beam has not been investigated, further measurements are required to measure its response compared with a photon beam. The gel can also offer potential advantages for validating treatment plans in BNCT, for example a boron loaded gel could be selectively introduced inside a normal gel in a head phantom to simulate a brain tumour in clinical conditions and a dose distribution produced for the tumour as well as healthy tissue. Alternatively the phantom could contain two boron loaded gels with different boron concentrations so that the dose to the tumour and blood vessels as well as normal brain tissue could be determined.

\subsection{Dose distribution from blood irradiator}

\subsubsection{Introduction}

The irradiation of blood and blood components is needed prior to transfusion to minimize the induction of graft versus host disease (GVHD) in high risk patients such as those receiving bone marrow grafts and intensive chemotherapy (Ramirez et al 1987). The irradiation of blood is aimed to eliminate relatively radiosensitive lymphocytes leaving other relatively radio-resistant blood components such as red cells and platelets. In order to kill lymphocytes with minimum effect on red cells, platelets 
and granulocytes, an absorbed dose of $25 \mathrm{~Gy}$ was recommended by the BSCH Blood Transfusion Task Force (1994), providing that no part receives more than $50 \mathrm{~Gy}$. The Task Force also recommended radiation as the only method for prevention of GVHD. Therefore centres involved in such work are normally equipped with a gamma irradiator. The results of a survey carried out by Anderson et al (1991) showed that $21.2 \%$ of blood centres, $19.1 \%$ of hospital blood banks and $8.5 \%$ of transfusion services had on site irradiation facilities. With commercially available irradiators the given doses are not uniform and can vary along the central axis (BCSH Blood Transfusion Task Force, Aukett 1994). Therefore validation of the dose delivered throughout the irradiation field to different points in the sample is necessary. The implementation of a quality assurance programme for the radiation process is also necessary, as the blood is prepared for patient use.

To date several methods have been used for dosimetric and calibration purposes including TLDs, Fricke dosimetry and ionization chambers (Anderson 1992). This section will discuss measurements of the absorbed dose distribution using the polymer gel irradiated by a gamma ray blood irradiator.

\subsubsection{Blood irradiators}

The majority of blood irradiation is often accomplished by irradiators that are designed specifically for this purpose. If such irradiators are not available, radiotherapy equipment can also be used to irradiate blood bags. The commercially available irradiators normally contain a gamma emitting source with a long half life. $\mathrm{Cs}^{137}$ is the isotope generally used as a source of radiation due to its longer half life and relatively less shielding requirement compared to that for $\mathrm{Co}^{60}$. Typical commercial irradiators use 1 to 4 sources of Caesium and the activity of each source ranges from 22 to $89 \mathrm{TBq}(600-2400 \mathrm{Ci})$. The nominal doserate for such irradiators at the time of installation varies from 3 to $4 \mathrm{~Gy} / \mathrm{min}$ to $10 \mathrm{~Gy} / \mathrm{min}$. The sources are linear and positioned vertically in the machine (Masterson and Febo 1992). 
Blood bags are normally irradiated inside a stainless steel container. The irradiation canister is positioned on a turntable which rotates in front of the radiation source during irradiation. The effect of turntable rotation during exposure is to improve the uniformity of dose distribution over the container volume.

The manufacturers supply a calibration certificate that provides central doserate and occasionally isodose distributions. The isodose distribution that is supplied by the manufacturer of the equipment used in this experiment shows a variation of \pm 10 to 20 $\%$ in doserate relative to the central doserate (figure 6.7).

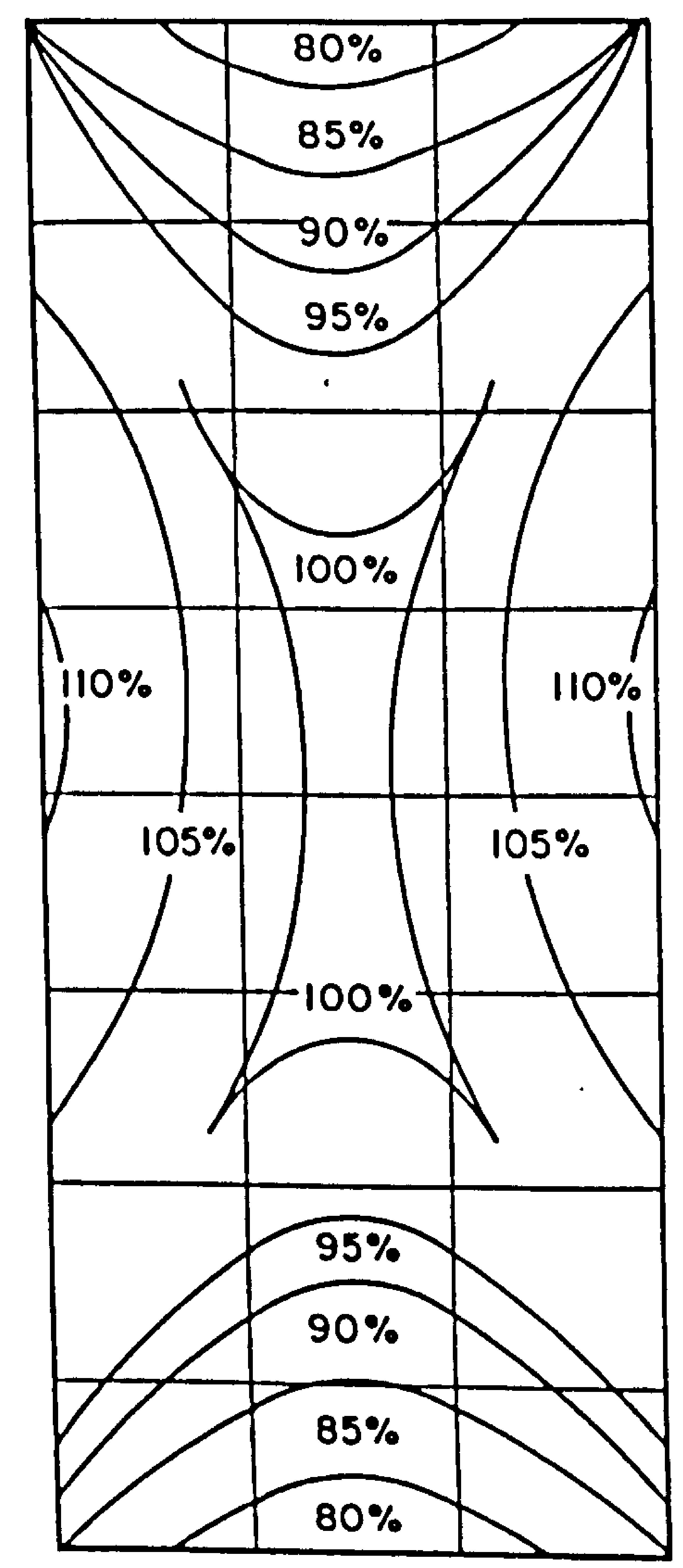

Figure 6.7. A typical isodose curve supplied by a manufacturer (Atomic Energy of Canada Ltd) 
A survey that has been carried out by Masterson and Febo (1992) showed a marked difference in doserate from those quoted by the manufacturers. Therefore an independent calibration is needed over the entire irradiation volume including both doserate and dose distributions. After installation of the irradiator the centre must be able to repeat the calibration after any malfunction of the machine, major repair or movement to a new location, and at least once a year to avoid under or overdosing of the blood samples.

\subsubsection{Measurement process}

BANG polymer gel was prepared using the same technique as described in chapter 3 (3.6.1). A cylindrical Perspex phantom of $12.5 \mathrm{~cm}$ height, $7.4 \mathrm{~cm}$ diameter and wall thickness of $3 \mathrm{~mm}$ was filled with the gel. The phantom was taken to the Haematology Department Leicester Royal Infirmary for irradiation in the Gammacell 1000 blood irradiator (Atomic Energy of Canada Ltd).

The Gammacell 1000 employs 1 pencil type Caesium-137 radioactive source supplied by Atomic Energy of Canada Ltd. The doserate quoted by the manufacturer was $2.73 \times 10^{4}$ Rads ( $273 \mathrm{~Gy}$ ) per hour at the centre of the chamber as measured by Ferrous sulphate dosimetry in 1988 (ACEL). The phantom was placed in the canister and the irradiation time was set to 2.4 minutes to give an absorbed dose of $8 \mathrm{~Gy}$. The set time was based on a time calculation that had been carried out previously (Aukett 1994). In order to calibrate the gel, 6 glass gel vials were irradiated to different known doses using a $6 \mathrm{MV}$ photon beam from a Philips SL 75/5 linear accelerator (3.7.4).

The gel was imaged four days after irradiation using the Siemens 1 T MRI scanner. The gel phantom was placed in the centre of the head coil and a multi slice spin-echo pulse sequence was applied. To produce $T_{2}$ weighted images, a TR of 6000 ms and TE of 15 and 400 ms were employed. A sagittal slice (parallel to pencil source) of $3 \mathrm{~mm}$ thick was taken throughout the gel. The field of view was $190 \mathrm{~mm}$ 
and matrix size was 256 . Using these parameters the time required to image the gel was half an hour.

To measure dose distribution the calculated $\mathrm{T}_{2}$ image was produced from the $\mathrm{T}_{2}$ weighted images. Then, by applying calibration data the $T_{2}$ values in each pixel were converted to dose using Analyze ${ }^{\mathrm{TM}}$ software running from a Sun Spark Station.

\subsubsection{Results and discussion}

The dose distribution produced in the gel by the blood irradiator is shown in figure 6.8. The data is normalised to the dose at the centre of the phantom. From the figure it can be seen that the dose in the central region of the gel is about $10 \%$ less than the maximum. The dose difference in the top and bottom of the gel is $30 \%$ less than that in the central region. The absorbed dose measured in the gel was $8.3 \pm 0.3$ Gy compared with the calculated value $8.0 \mathrm{~Gy} \pm 0.2$.

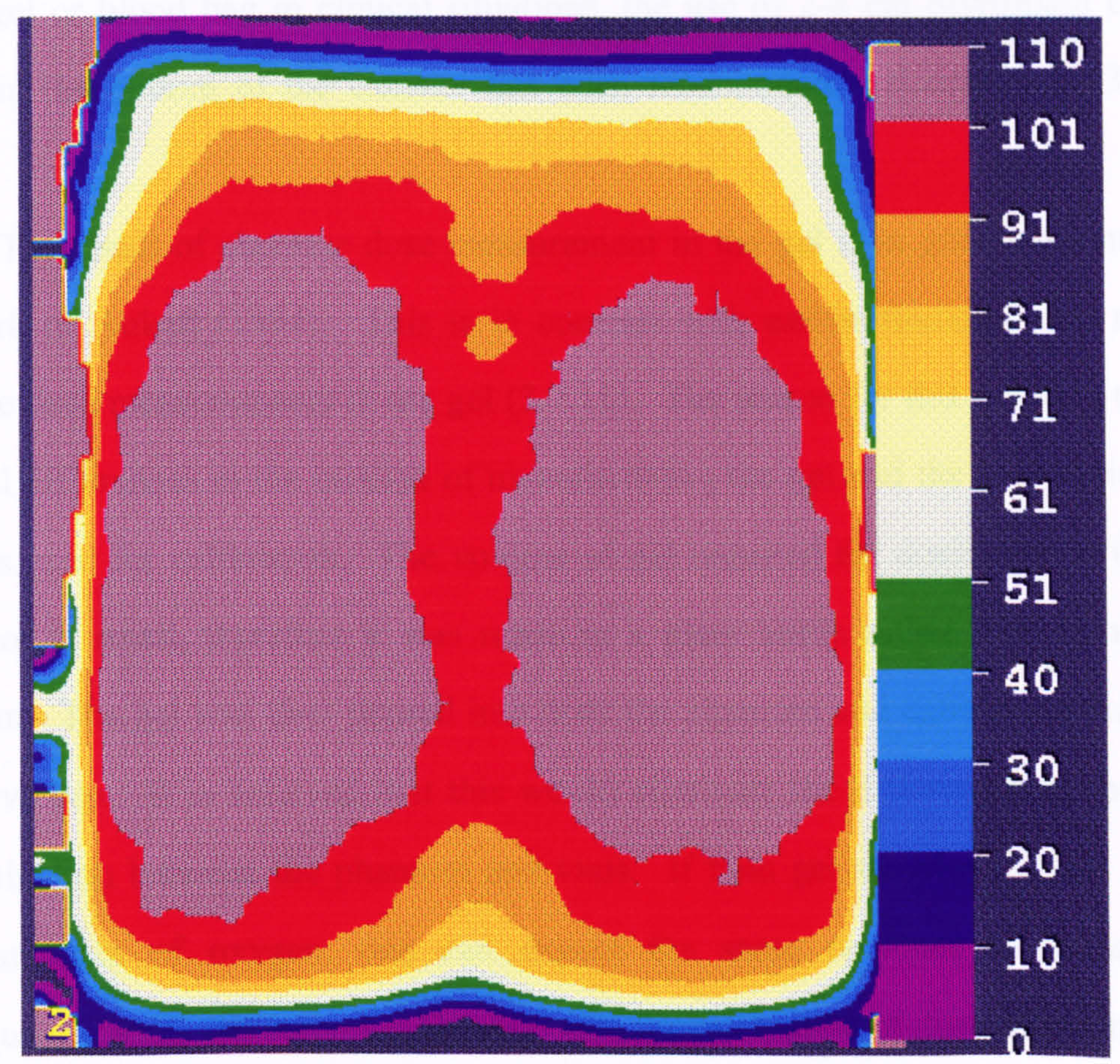

Figure 6.8. Absorbed dose distributions measured in the gel phantom. 
It has been shown that irradiation of blood is the only method for prevention of transfusion-induced graft-versus-host disease. There have however been some reports of patients developing GVHD following transfusion, even when the blood components have already been irradiated with the required dose (Leitman 1993). These cases point to the necessity of a regular quality assurance programme for the dedicated irradiator as well as verification of the dose distribution throughout the irradiation field to avoid inadequate blood irradiation.

The study of relative dose distribution using gel dosimetry showed a minimum dose of $70 \%$ both at the bottom and top of the gel. This is in agreement with the measurements carried out by Masterson and Febo (1992) using TLDs, but disagrees with the manufacturer's measurement. The manufacturer's isodose curve shows a minimum relative dose of $80 \%$. The highest dose measured in the gel relative to the central region is $115 \%$ compared to manufacturer results of $110 \%$ and Masterson and Febo (1992) results of $135 \%$. In order to deliver an adequate dose level to the bottom of the gel or blood bag in clinical situations, the use of $2-4 \mathrm{~cm}$ Styrofoam or plastic spacer in the bottom of the canister has been recommended (Leitman 1993, Aukett 1994).

The result of absolute dose measurement in the gel is in good agreement with the work of Aukett (1994). This is in contrast with earlier findings with regard to absolute dose measurements in the gel (3.7.11). The reason for this is likely to be due to small differences in the method of manufacturing the gel and the preparation of the samples used for calibration. The volume of gel required for dosimetry in the blood irradiator is small, therefore it was made in a glass bottle rather than in a Perspex phantom. The gel was then poured into both the phantom and calibrating vials inside the glove box. It is believed that this would eliminate the differences in the oxygen contamination between the phantom and vials. If both gel samples contain the same trace amounts of oxygen, one can expect the same response to radiation and consequently apply the correct calibration data for calculation of dose distribution. 
To date TLD dosimetry has been the method most frequently used to measure doserate and isodose levels in blood irradiators. It was claimed TLDs were used because they could provide good spatial resolution and negligible energy dependence in the energy range that is used (Masterson and Febo 1992). From the results of this study it can be concluded that polymer gel dosimetry could be a suitable method for calibrating and measuring the absorbed dose distribution from blood irradiation. The tissue equivalency, higher resolution (in this study pixel size was $0.74 \mathrm{~mm}$ ), doserate and energy independence, ease of three dimensional measurement and its non-invasive nature make it an excellent candidate for these purposes. 


\section{Chapter 7. Conclusions and future work}

\subsection{Conclusions}

Current developments in radiotherapy techniques have the aim of improving the quality of patient treatment but also make treatment planning more complex and consequently dose delivery more liable to error. These techniques, such as intensity modulated radiotherapy (IMRT), produce complex dose distributions in three dimensions. It is therefore necessary to verify these dose distributions in 3-D in a tissue equivalent phantom to increase confidence in the methods of dose delivery. Recently interest has been raised in the field of radiotherapy dosimetry using gel based systems. The BANG polymer gel is probably the best dosimeter of this kind produced to date. This system is based on polymerization and cross-linking of acrylic monomers in an aqueous gel by the action of radiation. These changes can then be measured using MRI.

The investigation of the main properties of BANG polymer gel, that were described in chapter 3 , explored the different features that are required for a dosimeter. The gel was found to be quite homogeneous after gelation, tissue equivalent and its' response was reproducible using the same batches of chemicals. The gel response was also found to be independent of energy over the range 0.3 to $8 \mathrm{MV}$ and doserate independent from 1.6 to $7.62 \mathrm{~Gy} \mathrm{~min}^{-1}$; which had not previously been quantified. The gel sensitivity was found to be dependent on the concentration of the total monomer using equal amount of bis and acrylamide. The higher fraction by weight of bis resulted in increased dose linearity, but the increased fraction by weight of acrylamide did not offer any advantages. It was quantitatively confirmed that once the polymerization has been produced in the gel the distribution is stable and does not diffuse throughout the gel, as had been found previously for ferrous sulphate based gels. Small changes in the transverse relaxation rate $\left(R_{2}\right)$ values were observed two days after irradiation indicating the possible presence of long lived free radicals. It has 
also been postulated that oxygen diffusion through or coming from the material used for the phantom wall has a strong influence on the polymerization process and consequently the dose distribution (chapters 4 and 5). The calibration of the gels was also found to be susceptible to oxygen contamination and temperature during MR imaging. Therefore very stringent controls are required for absolute dose measurements. However for relative measurements there is no need for gel calibration other than to confirm linearity and the background $R_{2}$ value can be measured either in the phantom or in a separate gel vial. This study also highlighted that BANG polymer gel is not sensitive to RF inhomogeneities using a head coil and the Hahn spin echo pulse sequence. An increased MR field strength of $1.5 \mathrm{~T}$ did not result in increased gel sensitivity compared to $1 \mathrm{~T}$.

Once the main properties of the gel had been determined the question to be answered was how useful would the dosimeter be in radiotherapy measurements. The results obtained in chapter 4 and 5 have demonstrated the application of BANG polymer gel in brachytherapy and external beam therapy. The high spatial resolution offered by the gel makes it a suitable candidate for brachytherapy measurements where the absorbed dose can decrease quite rapidly. The more obvious advantages of the gel are, its three dimensional nature, being the dosimeter as well as phantom and also its ability to take any configuration depending on the shape of the container. The latter made it possible to apply the gel successfully to a complex geometry simulating real clinical conditions in brachytherapy, though oxygen from the phantom material inhibited the dose measured in some regions. It is concluded that BANG polymer gel can play a useful role in brachytherapy particularly in the measurements of relative dose distributions. For absolute dose measurements particular attention must be paid to the method of calibration.

Initial results of depth dose measurements showed good agreement between the gel and ion chamber therefore the gel can be used for measuring both depth doses and beam profiles. The overall uncertainty in measuring dose was estimated to be within \pm $5 \%$. For simple measurements gel is not recommended as it is rather expensive (a litre 
of the gel costs abut $£ 15$ ) and takes more time than using ion chamber or diodes. For complex measurements requiring high resolution in three dimensions the gel is the best dosimeter available at present as it can image and measure dose distribution in any plane with high spatial resolution. The minimum in-plane resolution used in this study was $0.74 \mathrm{~mm}$.

It has been shown that the dose distributions from standard radiotherapy techniques produced in the gel not only agree with the basic dose distributions produced by Helax-TMS but show that the gel is able to highlight deficiencies in the algorithm, such as lack of backscatter. The gel performance for measurements using an air gap in the phantom was quite encouraging. The problem with this measurement was the non-uniform gel sensitivity due to the oxygen diffusion through the Perspex tubing used to form the cavity. This is the first time that the results from a gel dosimetry have been directly compared to the predictions of a 3-D planning system.

In conformal therapy the dose distribution from 5 intensity modulated radiation fields, using customized compensators was measured by the gel. The results compared well with that of radiographic film at the $90 \%$ isodose level. In comparison with the calculated distribution both methods showed similar discrepancies particularly in the left anterior oblique and the posterior region. This indicates that both a more accurate dose delivery and some further modifications to the calculations are required.

The measurement of dose distribution from a dynamic wedge has indicated that gel dosimetry can be a more flexible and faster method for such a measurement than diodes, since it is a integrating dosimeter that provides data in any required plane with higher resolution. Further work is required to compare the gel data with measurements using a diode array.

In general it is believed that the gel not only could be used for verification of more complex treatment plans but also its stability can make it a suitable candidate for transportation for purposes of inter-department audit.

The application of gel dosimetry to a specialised method of radiation therapy, boron neutron capture therapy (BNCT), was described in chapter 6. Boron loaded gel 
has shown a minimum of $50 \%$ more dose than pure gel for a concentration of $60 \mathrm{ppm}$ of ${ }^{10} \mathrm{~B}$. Therefore by comparing the results of the boron loaded and pure gel the contribution of the boron capture to the absorbed dose can be determined. It is also believed that the gel can potentially be used for validation of treatment plans in BNCT provided that its response to heavy particles can be determined. This is a novel application of gel dosimetry. The ability of the gel to measure dose distribution in restricted areas, such as a blood irradiator was also described in chapter 6 . This is also a new application of the gel. The results suggested that the gel could be a suitable dosimeter for both calibrating and measuring dose distributions produced for blood irradiation.

The main drawbacks of the use of BANG gel are that, it can only be used once and currently dose measurements require MRI. This makes the dosimetry rather expensive. However if a stack of films were used costs are comparable. An alternative approach to measuring dose distributions in the gel could be the tomographic optical scanning system (Gore et al 1996), currently under development which may provide a relatively low cost measurement system. This system is in the early stage of its development and further work is required to improve its performance. Monomer powders are also toxic and the manufacturing process requires some special safety precautions. Finally absolute dose measurement requires standard methods of gel manufacturing and calibration to avoid alteration in gel sensitivity due to differences in oxygen contamination. The polymer gel, however has the potential of solving many of current problems associated with dosimetry for modern radiotherapy and is an excellent candidate for dosimetry of complex geometries and for use in restricted areas.

\subsection{Future work}

The work presented in this thesis has provided basic information on the main properties of BANG gel and confirmed some data reported in the literature. It has also 
high-lighted some practical aspects of its application in different areas of radiotherapy. However there is still much to be done in the future. This includes-

Improvements in the method of gel manufacturing need to be developed, with the aim of ensuring the consistency of results. The rate of oxygen diffusion from Perspex material should be explored. This would enable investigators to use Perspex phantoms more efficiently. The investigation of other plastics with low rate of oxygen diffusion as a phantom material should be carried out. One such material would be Barex (BP chemical) with a reported oxygen diffusion rate of $0.3 \mathrm{~cm}^{3} \cdot \mathrm{mm} \cdot \mathrm{m}^{-2}$.day 1.bar ${ }^{-1}$ which is a factor of 10 less than polyvinyl chloride (PVC). It would also be of interest to see if the gel composition could be modified to simulate other tissue such as lung or bone. The use of gel phantoms in audit, particularly techniques such as treatment of breast should be explored. The determination of gel response to high LET radiation and further confirmation of the BNCT results is required. This could lead to the development of a phantom to use in comparisons with Monte Carlo calculations. Finally the gel will be a valuable tool to aid verification of dose distributions produced by dynamic muti-leaf collimators.

\subsection{Practical considerations}

The following factors should be taken into account in polymer gel dosimetry. 1. Use only glass components in the manufacturing to avoid Oxygen contamination. 2. Avoid prolonged nitrogenation in the last stage of gel manufacturing. This can cause spontaneous polymerization.

3. Transferring of gel solution from the vessel to the phantom should be carried out in a nitrogen atmosphere.

4. Maintain a constant gel temperature during MRI imaging. Room temperature is the easiest to achieve.

5. Maintain constant conditions for both calibration samples and irradiated gel.

6. Use the highest magnetic field strength available to maximise signal-to-noise ratio. 
7. Use Hahn spin-echo to make the measurements more reliable.

8. The in-plain resolution and slice thickness should be chosen to match the geometry of the phantom.

9. Position the gel in the centre of the RF coil to ensure the maximum RF field uniformity. 


\section{Chapter 8. References}

Ablett S., Lillford P.J., Baghdadi S.M.A., and Derbyshire W., (1978). Nuclear magnetic resonance investigations of polysaccharide films, sols, and gels. J. Colloid. Inter. Sci 67(2), 355-377

Allen D.A., and Beynon (1995). A design study for an accelerator-based epithermal neutron beam for BNCT. Phys. Med. Biol. 40, 807-821

Anderson G., (1992). Quality assurance of the irradiation process of blood components. in: Irradiation of blood components edited by Baldwin and Jefferies. American Association of Blood Banks

Anderson K.C., Goodnough L.T., Sayers M., Pisciotto P.T., Kurtz S., Lane T.A., Anderson C.S., and Silberstein L.E., (1991). Variation in blood component irradiation practice: implications for prevention of transfusion-associated graft-versus-host disease. Blood 77, 2096-2102

Appleby A., Christman E.A., and Leghrouz A. (1987). Imaging of spatial radiation dose distribution in agarose gels using magnetic resonance. Med. Phys. 14(3), 382384

Appleby A., Leghrouz A. and Chistman E.A. (1988). Radiation chemical and magnetic resonance studies of aqueous agarose gels containing ferrous ions. Radiat. Phys. Chem. 32, 241-244

Atomic Energy of Canada Limited (ACEL), 1988. Certificate of measurement, Gammacell 1000, No 146. 
Audet C., Duzenli C., Kwa W., Tsang V., and Mackay A. (1996). An example of MRI polymer gel dosimetry applied to 3D conformal radiotherapy. Med. Phys 23(5), 803

Audet C., Maryanski M.J., and Gore J.C., (1995). Dose response of the BANG polymer gel dosimeter: Dependence on composition. Med. Phys 22(6), 951

Aukett R., (1994). Report of dose measurements at different points in the blood bag and timing calibration. LRI internal report, Leicester Royal Infirmary, Medical Physics

Balcom B.J., Lees T.J., Sharp A.R., Kulkarni N.S., and Wagner G.S. (1995). Diffusion in $\mathrm{Fe}(11 / 111)$ radiation dosimetry gels measured by magnetic resonance imaging. Phys. Med. Bio. 40, 1665-1676

Baldock C., Burford R.P., Billingham N.C., Cohen D., and Keevil S.F., (1996c). Polymer gel composition in magnetic resonance imaging dosimetry. Med. Phys. 23(6), 1070

Baldock C., Burford R.P., Billingham N., Wagner G.S., Patval S., Badawi R.D., and Keevil S.F., (1998). Experimental procedure for the manufacture and calibration of polyacrylamide gel (PAG) for magnetic resonance imaging (MRI) radiation dosimetry. Phys. Med. Bio. 43, 695-702

Baldock C., Hasler C., Keevil S.F., Greener A.G., Billingham N.C., and Burford R., (1996b). A dosimetry phantom for external beam radiation therapy of the breast using radiation-sensitive polymer gels and MRI. Med. Phys 23(8), 1490

Baldock C., Hindocha N., Billingham N.C. Burford R. Keevil SF., and Procter E. (1996a). Dosimetry of a new device for interstitial radiosurgery using radiation sensitive polymer gels and MRI. Med. Phys 23(8), 1490 
Battista J.J., Rider W.D., and Dyk J.V., (1980). Computed tomography for radiotherapy planning. Int. J. Radiat. Oncol. Biol. Phys 6, 99-107

Baustert I.C., Oldham M., Smith T.A.D., Webb S., and Leach M.O., (1997). R2 images used for isodose plots in conformal tomotherapy. Preceding of the International Society for Magnetic Resonance in Medicine. Vancouver, Canada.

BCSH Blood Transfusion Task Force (1994). Guidelines on gamma irradiation of blood components for the prevention of transfusion-associated graft-versus-hostdisease. Fifth draft.

Bengtsson M., Furre T., Rødal J., Skretting A., and Olsen D.R., (1996). Measurement of dynamic wedge angles and beam profiles by means of MRI ferrous sulphate gel dosimetry. Phys. Med. Bio. 41, 269-277

Bidmead A.M., Garton A.J., and Childs P.J. (1995). Beam data measurements for dynamic wedges on Varian $600 \mathrm{C}(6 \mathrm{MV})$ and $2100 \mathrm{C}$ (6 and $10 \mathrm{MV}$ ) linear accelerators. Phys. Med. Bio. 40, 393-411

Bini M.G., Ignesti A., Millanta L., Olmi R., Rubino N., and Vanni R. (1984). The polyacrylamide as a phantom material for electromagnetic hyperthermia studies. IEEE Trans. Biomed. Eng. 31(3), 317-322

Bio-rad Bulletin (1987). Acrylamide polymerization-a practical approach. Bio-rad Bulletin 1156, Richmond, CA, Bio-rad Laboratories, USA

Bonnett D.E., Farajollahi A.R., Haas O., Mills J.A., Glendinning A.G., Aukett R.J., and Burnham K (1997). The verification of intensity modulated conformal therapy using polymer gel dosimetry. Proc World Cong. Med. Phys. Bio. Eng., Nice, P 1012 
Brahme A. (1987). Design principles and clinical possibilities with a new generation of radiation therapy equipment. A review. Acta. Oncol. 26(6), 403-412

Brahme A., Lind B., and Källman P. (1990). Inverse radiation therapy planning as a tool for 3D dose optimization. Physica Medica. 6(2), 53-67

Bucciolini M., Milano F., Pallotta S., and Renzi R., (1991). Dose distributions in tissue equivalent phantom measured by magnetic resonance imaging. Physica medica, $7(3), 125-131$

Burch S.E., Kearfott K.J., Trueblood J.H., Sheils W.C., Yeo J.I., and Wang C.K.C., (1997). A new approach to film dosimetry for high energy photon beams: lateral scatter filtering. Med. Phys 24(5), 775-783

Cantone M.C., Canzi C., Cerchiari U., deBartolo D., Facchielli L., Gambarini G., Molho N., Pirola L. and Sichirollo A.E. (1992). A ferrous sulphate gel dosimetry system for NCT studies: Response to slow neutrons. In Neutron Dosimetry, Proc. 7th Symp. Berlin, Fed. Rep. Germany 1991. Radiation Protection Dosimetry, 44(1-4), $437-441$

Carr H. Y., and Purcell E. M. (1954). Effects of diffusion on free precession in nuclear magnetic resonance experiments. Physical Review 94(3), 630-638

Cassell K.J., Hobday P.A., and Parker R.P. (1981). The implementation of a generalised Batho inhomogeneity correction for radiotherapy planning with direct use of CT numbers. Phys. Med. Bio. 26(4), 825-833

Chakeres D.W and Schmalbrock P, (1992). Fundamentals of Magnetic Resonance Imaging. Williams \& Wilkins, USA 
Chan M.F., and Ayyanga K.R. (1993). Verification of water equivalence of FeMRI gel using Monte Carlo simulation. Med. Phys. 20(3), 901

Chan M.F., and Ayyangar K.M. (1995). Confirmation of target localization and dosimetry for $3 \mathrm{D}$ conformal radiotherapy treatment planning by MR imaging of a ferrous sulphate gel head phantom. Med. Phys. 22(7), 1171-117.

Chapiro A., (1962). Radiation chemistry of polymeric systems. John Willy \& Sons, Inc, New York.

Chapiro A., (1967). Chemical nature of the reactive species produced in polymers by ionising radiations. In: Irradiation of polymers. American Chemical Society, Washington, D.C.

Charlesby A., (1987). Radiation chemistry of polymers. in: Radiation chemistry: Principles and Applications. Edited Farhataziz and M.A.J. Rodgers. VCH Publishers, Inc, USA.

Charlesby A., (1991). The effects of ionising radiations on polymers. in: Irradiation effects on polymers 39-78. Elsevier Applied Science, London.

Cheng C., and Das I.J., (1996) Dosimetry of high energy photon and electron beams with CEA films. Med. Phys 23(7), 1225-1232

Chrambach A., and Rodbard D., (1983). "Quantitative" and preparative polyacrylamide gel electrophoresis. In: Gel electrophoresis of proteins. A practical approach. edited by Hames B.D., and Rickwood D., IRL Press Limited., Oxford. 
Coffey C.W., Hines H.C., Wang P.C., and Smith S.L. (1984). The early applications and potential usefulness of NMR in radiation therapy treatment planning. In: Proceedings of 8th International Conference on the Use of Computers in Radiation Therapy, Toronto, Canada, 173-180.

Constantinou C., (1978). Tissue substitutes for particulate radiations and their use in radiation dosimetry and radiotherapy. Ph.D. thesis, London University

Constantinou C., (1982). Phantom materials for radiation dosimetry. I. Liquids and gels. B.J.R 55, 217-224

Constantinou C., Attix F.H., and Paliwal B.R., (1982). A solid water phantom material for radiotherapy $\mathrm{x}$-ray and $\gamma$-ray beam calibrations. Med. Phys 9(3), 436-441

Day M.J., (1990). Radiation dosimetry using nuclear magnetic resonance: an introductory review. Phys. Med. Bio. 35(12), 1605-1609

deGuzman A., Gore J.C., and Schulz R.J., (1989). Dose response curves for gels infused with Fricke dosimeter by NMR. Med. Phys. 16(3), 457

Dewhurst H.A., (1951). Effect of organic substance on the $\gamma$-ray oxidation of ferrous sulphate. J. Chem. Phys. 19, 1329

Djennaui N., (1994). Measurement of radiotherapy dose distributions using dosimeter gels and MRI. Ph.D. Thesis. University of Leeds

Duzenli C., Audet C., Strgar V., Kwa W., MacKay A., and Blackmore E., (1997). Proton beam dosimetry: Mini chamber. Radiochromic film and polymer gel. Med. Phys. 24(7), 1202 
Duzenli C., Sloboda R., and Robinson D. (1994). A spin-spin relaxation rate investigation of the gelatine ferrous sulphate NMR dosimeter. Phys. Med. Bio. 39, $1577-1592$

Edwards M., Keller J., Larsen G., Rowberg A., Sandler B., and Whitaker F., (1981). A computed tomography-radiation therapy treatment planning system utilizing a whole body CT scanner. Med. Phys, 8(2), 242-248.

Elder P.J., Coveney F.M., and Welsh A.D., (1995). An investigation into the comparison between different dosimetric methods of measuring profiles and depth doses for dynamic wedges on a Varian 600C linear accelerator. Phys. Med. Bio. 40, $683-689$

Farajollahi A.R., Bonnett D.E., Aukett R.J., and Ratcliffe A.J., (1997) The advantages and limitations of polymer gel dosimetry in brachytherapy. Proc World Cong. Med. Phys. Bio. Eng., Nice, P 840

Fricke H. and Hart E.J., (1972). Chemical dosimetry. In: Radiation dosimetry, 167197 Vol. II, Edited Attix F.H. and Roesh W.C., Academic press, New York.

Gabel D., (1996), Progress in the field of compounds for boron neutron capture therapy. In Hardons in radiation therapy. Proceedings of the meeting of the European Heavy Particle Therapy Group. Orleans (France), 4-6 May 1995.

Gambarini G., Arrigoni S., Cantone M.C., Molho N., Facchielli L. and Sichirollo A.E. (1994). Dose-response curve slope improvement and result reproducibility of ferroussulphate-doped gels analysed by NMR imaging. Phys. Med. Bio. 39, 703-717 
Geise R., and McCullough E.C., (1977). The use of CT scanners in megavoltage photon-beam therapy planning. Radiology, 124, 133-141

Gibbs P., Beavis A.W., Dealey R.A., Whitton V.J., and Horsman A., (1997). MRI based radiotherapy planning of the brain. Preceding of the International Society for Magn. Reson. Med. Vancouver, Canada, 1117.

Godden T.J., (1988). Physical aspect of brachytherapy. Medical Physics Handbooks 19. Adam Hilger, Bristol.

Gore J.C., (1983). Physical principles of nuclear magnetic resonance. in: Recent advances in radiology and medical imaging, No 7, Steiner.

Gore J.C., Kang Y.S., and Schulz R.J., (1984a). Measurement of radiation dose distributions by nuclear magnetic resonance (NMR) imaging. Phys. Med. Bio. 29(10), 1189-1197

Gore J.C., Kang Y.S., and Schulz R.J., (1984b). Measurement of radiation dose distributions by NMR imaging. Magn. Reson. Imaging. 2, 244

Gore J.C., Ranade M., Maryanski M.J., and Schulz R.J. (1996). Radiation dose distributions in three dimensions from tomographic optical density scanning of polymer gel: I. Development of an optical scanner. Phys. Med. Bio. 41, 2695-2704

Gupta N., Niemkiewicz J., Blue T.E., Gahbauer R., and Qu T.X., (1993) Effect of head phantom size on $10 \mathrm{~B}$ and $1 \mathrm{H}[\mathrm{n}] ,2 \mathrm{H}$ dose distributions for a broad field accelerator epithermal neutron source for BNCT. Med. Phys 20(2), 395-404

Hahn E.L. (1950). Spin echoes. Physical Review 80(4), 580-594 
Hames B.D., (1983). An introduction to polyacrylamide gel electrophoresis. In: Gel electrophoresis of proteins. A practical approach. edited by Hames B.D., and Rickwood D., IRL Press Limited., Oxford.

Hardy P. A., Bronskill M. J., and Henkelman R. M., (1985). Signal strength on a 0.15-T magnetic resonance imager. Med. Phys. 12 (5) 581-585

Hass O., (1997) Optimisation and control systems modelling in radiotherapy treatment planning. Ph.D. Thesis, Coventry University

Hazle J.D., Hefner L., Nyerick C.E., Wilson L., and Boyer A.L. (1991). Doseresponse characteristics of a ferrous-sulphate-doped gelatine system for determining radiation absorbed dose distributions by magnetic resonance imaging (Fe MRI). Phys. Med. Bio. 36(8) 1117-1125.

Hefner L.F., Hazle J.D., Otte V.A., and Boyer A.L., (1992). Single field depth dose characteristic measured using ferrous sulphate gels and MRI. A comparison with film and ion chamber measurements. Med. Phys. 19(3), 774

Hejira N., Raimondi S., Tercier PA., and Valley J.F., (1998) A qualitative study of the performances of treatment planning systems. To be published

Hiraoka T., Hoshino K., Kawashima K., and Kato H. (1993). A new gel using super absorbent polymer for mapping the spatial dose distributions of electron beams by MR imager. Medical Dosimetry. 18, 73-79

Hoecker F.E., and Watkins I.W., (1958). Radiation polymeization dosimetry. International Journal of Applied Radiation and Isotopes 3, 31-35 
Horsfield M.A, (1998). Standardisation, optimisation and organisation of MRI for monitoring clinical trials. In: Magnetic Resonance Techniques in clinical trials in multiple sclerosis. Edited by Filippi, Grossman and Comi. Springer Verlag (to be published)

Howe F. (1988). Relaxation times in paramagnetically doped agarose gels as a function of temperature and ion concentration. Magn. Reson. Imaging. 6, 263-270

Ibbott G.S., Bova F.J., Maryanski M.J., Zhang Y., Holcomb S., Avison R.G., and Meeks S.L., (1996). Use of BANG polymer gel dosimeter to evaluate repeat-fixation stereotactic radiation therapy. Med. Phys 23(6), 1070

Ibbott G.S., Maryanski M.J., Avison R.G., and Gore J.C., (1995). Investigation of BANG polymer gel dosimeter for use as a mailed QA device. Med. Phys, 22(6), 951

ICN Biochemicals. MSDS. Material safety data sheet.

Joint Working Party of the BIR and the IPSM, (1992). Recommendations for brachytherapy dosimetry. $B I R$

Katayama S., and Fujiwara S., (1979). NMR study of the spatial effect of polyacrylamide gel upon the water molecules confined. Int. J. Am. Chem. Soc. 101(16), $4485-4488$

Kean D.M., and Smith M.A., (1986). Magnetic Resonance Imaging: Principles and Applications, 6-49 Heinemann, London. 
Kennan R.P., Maryanski M.J., Zhong J., and Gore J.C., (1992). Hydrodynamic effects and cross relaxation in cross linked polymer gels. Preceding of the 11th symposium of the Society of Magnetic Resonance in Medicine, Berlin.

Kennan R.P., Richardson K.A., Zhong J., Maryanski M.J., and Gore J.C., (1996). The effects of cross-link density and chemical exchange on magnetization transfer in polyacrylamide gels. Journal of Magnetic Resonance. Series B 110, 267-277

Kessler M.L., Pitluck S., Petti P., and Castro J.R., (1991). Integration of multimodality imaging data for radiotherapy treatment planning. Int. J. Radiat Oncol Biol Phys. 21, 1653-1667

Kijeweski P.K., and Bjärngard B.E., (1978). The use of computed tomography data for radiotherapy dose calculations. Int. J. Radiation Oncology. Biol. Phys 4, 429-435

Kijewski P.K., Chin L.M., and Bjärngard B.E., (1978). Wedge-shaped dose distributions by computer-controlled collimator motion. Med. Phys 5(5), 426-429.

Kjær L., Thomsen C., Henriksen O., Ring P., Stubgaard M., and Pedersen E. J., (1987). Evaluation of relaxation time measurements by Magnetic Resonance Imaging. A phantom study. Acta Radiologica 28 345-351

Kjos B. O., Ehman R. L., and Zawadzki M. B., (1985). Reproducibility of $T_{1}$ and $T_{2}$ relaxation times calculated from routine MR imaging sequences: Phantom study. AJR. 144. 1157-1163

Klevenhagen S.C., Aukett R.J., Harrison R.M., Moretti C., Nahum A.E., and Rosser K.E., (1996). The IPEMB code of practice for the determination of absorbed dose for 
x-rays below $300 \mathrm{kV}$ generating potential (0.035 mm Al-4 mm Cu HVL; 10-300 kV generating potential). Phys. Med. Bio. 41, 2605-2625.

Kron T., Metcalfe P. and Pope J.M. (1993). Investigation of the tissue equivalence of gels used for NMR dosimetry. Phys. Med. Bio. 38, 139-150

Kron T., and Pope J.M. (1994). Dose distribution measurements in superficial X-ray beams using NMR dosimetry. Phys. Med. Bio. 39, 1337-1349

Land N. (1994). An investigation into the use of Fricke-infused agarose gel and nuclear magnetic resonance imaging to determine dose distributions. M.Sc. thesis, University of Birmingham

Landberg T.G., (1995). Progress in radiotherapy. Acta. Oncol, 34(8), 1023-1029

Leach M.O., (1988). Spatially Localised Nuclear Magnetic Resonance. in: The Physics of Medical Imaging. Edited by S. Webb., Medical Science Series

Leavitt D.D., and Larsson L., (1993). Evaluation of a diode detector array for measurement of dynamic wedge dose distributions. Med. Phys 20(2), 381-382

Leitman S.F., (1993). Dose, dosimetry, and quality improvement of irradiated blood components. Transfusion 33(6), 447-449

Lerski R.A., (1985). Principles of nuclear magnetic resonance. in: Physical principles and clinical applications of Nuclear Magnetic Resonance. Edited by Lerski R.A.. Paradigm print, Gsteshead, 59-75 
Lichter A.S., Fraass B.A., and Mcshan D.L. (1988). Recent advances in radiotherapy treatment planning. Oncology 131-133

Lillicrap S.C., Owen B., Williams J.R., and Williams P.C., (1990). Code of practice for high-energy photon therapy dosimetry based on the NPL absorbed dose calibration service. Phys. Med. Bio. 35(10), 1355-1360.

Lind B., and Källman P. (1990). Experimental verification of an algorithm for inverse radiation therapy planning. Radiother. Oncol. 17, 359-368

Liu H.H., Lief E.P., and McCullough E.C., (1997). Measuring dose distributions for enhanced dynamic wedges using a multichamber detector array. Med. Phys 24(9), $1515-1519$

Loeffler W.K., (1990). Magnetic Resonance Imaging. in: 3D imaging in Medicine. Edited by Höhne et al. Springer-Verlag Berlin.

Low D.A., and Mutic S., (1997). Abutment region dosimetry for sequential arc IMRT delivery. Phys. Med. Bio. 42, 1465-1470

Luciani A.M., Di Capua S., Guidoni L., Ragona R., Rosi A., and Viti A. (1996). Multiexponential T2 relaxation in Fricke agarose gels: Implications for NMR dosimetry. Phys. Med. Bio. 41, 509-521

Makhlis F.A., (1975). Radiation Physics and Chemistry of Polymers. Wiley, London.

Mansfield P., and Morris P.G., (1982). NMR imaging in Biomedicine. Academic Press, INC London. 
Martin J.D., Lehmkuhl R.V., Ibbott G.S., Meigooni A.S., and Maryanski M.J., (1997) HDR source characterization using the BANG gel dosimeter. Med. Phys. 24(6), 1062

Maryanski M.J., (1997). Private communication

Maryanski M.J., Audet C., and Gore J.C. (1995). Dose response of BANG polymer gel dosimeter: Temperature dependence. Med. Phys 22(6), 951

Maryanski M.J., Audet C., and Gore J.C. (1997a). Effects of cross linking and temperature on the dose response of a BANG polymer gel dosimeter. Phys. Med. Bio. $42,303-311$

Maryanski M.J., and Gore J.C., (1992). Flip angle variations in large volumes of dielectric and conductive media at high fields: Implications for quantitation of relaxation times from images. Proceedings of 11th Scientific Meeting of Society of Magn. Reson. Med. Berlin, 1349

Maryanski M.J., Gore J.C., Kennan R.P. and Schulz R.J. (1993). NMR relaxation enhancement in gels polymerized and cross-linked by ionisation radiation: A new approach to 3D dosimetry by MRI. Magn. Reson. Imaging. 2, 253-258

Maryanski M.J., Ibbott G.S., Eastman P., Schulz R.J., and Gore J.C. (1996a). Radiation therapy dosimetry using magnetic resonance imaging of polymer gels. Med. Phys 23(5), 699-705

Maryanski M.J., Kennan R.P., Gore J.C., and Hafeli U., (1997b). High-resolution 3D dosimetry for endovascular bracheythrapy using high-field MRI micro imaging of BANG polymer. Med. Phys. 24(6), 995 
Maryanski M.J., Schulz R.J., Ibbott G.S., Gatenby J.C., Xie J., Horton D. and Gore J.C. (1994). Magnetic resonance imaging of radibrachytherapystributions using a polymer-gel dosimeter. Phys. Med. Bio. 39, 1437-1455

Maryanski, M.J., Zastavker Y.Z., and Gore J.C., (1996b). Radiation dose distributions in three dimensions from tomographic optical density scanning of polymer gels: II. Optical properties of the BANG polymer gel. Phys. Med. Bio. 41, 2705-2717

Masterson M.E., and Febo R., (1992). Pre transfusion blood irradiation: Clinical rationale and dosimetric considerations. Med. Phys 19(3), 649-657

McCullough E.C., and Holmes T.W., (1985). Acceptance testing computerized radiation therapy treatment planning systems: Direct utilization of CT scan data. Med. Phys. 12(2), 237-242

Meiboom S., and Gill D. (1958). Modified spin-echo method for measuring nuclear relaxation times. The Review of Scientific Instruments 29(8), 688-691

Metcalfe P.E., Hoban P.W., Harper N.R., Murray D.C., and Round W.H., (1990). The production of body analogs for use in radiation physics. Austral. Phys. Eng. Sci. Med $13,117-128$

Mills J, (1996) . Private communication

Mitchell M.D., Kundel H.L., Axel L., and Joseph P.M. (1986). Agarose as a tissue equivalent phantom material for NMR imaging. Magn. Reson. Imaging. 4, 263-266

Morton-Jones D.H., (1989). Polymer processing. Chapman and Hall USA. 
Nicholson J.W., (1991). The Chemistry of polymers. The Royal Society of Chemistry, Cambridge.

Nigg D.W., Randolph P.D., and Wheeler F.J. (1991). Demonstration of threedimensional deterministic radiation transport theory dose distribution analysis for boron neutron capture therapy. Med. Phys. 18(1), 43-53

Olsen D.R., and Hellesnes J., (1994). Absorbed dose distribution measurements in brachytherapy using ferrous sulphate gel and magnetic resonance imaging. B.J.R. 67, $1121-1126$

Olsson L.E., Appleby A., and Sommer J., (1991). A new dosimeter based on ferrous sulphate solution and agarose gel. Appl. Radiat. Isot. 42(11), 1081-1086

Olsson L.E., Petersson S., Ahlgren L., and Mattsson G., (1989). Ferrous sulphate gels for determination of absorbed dose distributions using MRI technique. Basic studies. Phys. Med. Bio. 34(1), 43-52

Olssen L.E., Westrin B.A., Fransson A. and Nordell B. (1992). Diffusion of ferric ions in agarose dosimeter gels. Phys. Med. Bio. 37, 2243-2252

Orton C.G., (1966). Clear Perspex dosimetry. Phys. Med. Bio. 11(3), 377-386

Parker R.P., Hobday P.A., and Cassell K.J., (1979). The direct use of CT number in radiotherapy dosage calculations for inhomogeneous media. Phys. Med. Bio. 24(4), 802-809 
Perks C.A., Mill A.J., Constantine G., Harrison K.G., and Gibson J.A.B. (1988). A review of boron neutron capture therapy (BNCT) and the design and dosimetry of a high-intensity, $24 \mathrm{KeV}$, neutron beam for BNCT research. B.J.R, 61, 1115-1126

Pignol J-P., Oudart H., Chauvel P., Sauerwein W., Gabel D., and Prevot G., (1998). Selective delivery of ${ }^{10} \mathrm{~B}$ to soft tissue sarcoma using ${ }^{10} \mathrm{~B}-\mathrm{L}$-borophenylalanine for boron neutron capture therapy. B.J.R, 71, 320-323

Podgorsak M.B. and Schreiner L.J, (1992). Nuclear magnetic relaxation characterization of irradiated Fricke solution. Med. Phys. 19(1), 87-95

Pöller F., Sauerwein W., and Rassow J., (1992). Determination of dose enhancement by neutron capture of ${ }^{10} \mathrm{~B}$ in a d(14) + Be neutron beam. In Neutron Dosimetry, Proc. 7th Symp. Berlin, Fed. Rep. Germany 1991. Radiation Protection Dosimetry, 44(1-4), $437-441$

Prasad P.V., Nalcioglu O., and Rabbani B., (1991). Measurement of threedimensional radiation dose distributions using MRI. Radiation Research, 128, 1-13

Raaijmakers C.P.J., Bruinvis I.A.D., Nottelman E.L., and Mijnheer B.J., (1998). A fast and accurate treatment planning method for boron neutron capture therapy. Radiother. Oncol, 46, 321-332

Rae W.I.D., Willemse C.A., Lötter M.G., Engelbrecht J.S., and Swarts J.C. (1996). Chelator effect on ion diffusion in ferrous-sulphate-doped gelatin gel dosimeters as analyzed by MRI. Med. Phys. 23(1), 15-23

Ramirez A.M., Woodfield D.G., Scott R., and McLachlan J., (1987). High potassium levels in stored irradiated blood. Transfusion 27(5), 444-446 
Ratclife A, (1997). Private communication

Righetti P.G., Gelfi C., and Bosisio A.B., (1983). Polymerization Kinetics of polyacrylamide gels: Effects of different cross-linkers, Temperature and Catalysts. In Electrophoresis '82, de Gruyter \& Co., Berlin, New York, P 147-156.

Roth K., (1984). NMR-Tomography and Spectroscopy in Medicine. An introduction. Springer-Verlag. $1-9$

Rousseau J., Gibon D., Sarrazin T.H., Doukhan N., and Marchandise X., (1994). Technical note. Magnetic resonance imaging of agarose gel phantom for assessment of three-dimensional dose distribution in linac radiosurgery. B.J.R. 67, 646-648

Schreiner L.J., Crooks I., Evans M.D.C., Keller B.M., and Parker W.A., (1994). Imaging of HDR Brachytherapy dose distributions using NMR Fricke-Gelatine dosimetry. Magn Reson Imaging. 12(6), 901-907

Schulz R.J., deGuzman A.F., Nguyen D.B., and Gore G.C., (1990). Dose-response curves for Fricke-infused gels as obtained by nuclear magnetic resonance. Phys. Med. Bio. 35(12), 1611-1622

Schulz R.J., Maryanski M.J., Ibbott G.S., and Bond J.E. (1993). Assessment of the accuracy of stereotactic radiosurgery using Fricke-infused gels and MRI. Med. Phys 20(6), 1731-1734

Shalev S., Manittoba Cancer Treatment and Research Foundation (MCTRF)., (1996). Portal Image Processing System. 
Sharrock C., and Read G., (1998). The present status of CRT. Rad Magazine March, $37-38$

Simmons A., Arridge S. R., Barker G. J., Cluckie A. J., and Tofts P. S., (1994). Improvements to the quality of MRI cluster analysis. Magn. Reson. Imaging. 12(8), $1191-1204$

Smajo M.E,. (1995). The feasibility of quantitative ferrous sulphate/agarose gel dosimetry: Comparison with ionisation chamber measurements for photons and electrons. Abstract of Ph.D. Dissertation, Rush University, Chicago. Med. Phys. 23(8), 1468 (1996)

Spinks J.W.T. and Woods R.J., (1964). An introduction to radiation chemistry. 105118., John Wiley and Sons, Sydney

Tattam D., (1997). Development and characterisation of an accelerator based BNCT beam. Ph.D. Thesis, The University of Birmingham

Taylor D. G. and Inamdar R., (1985). Principles of nuclear magnetic resonance. in: Physical principles and clinical applications of Nuclear Magnetic Resonance. Edited by Lerski R.A.. Paradigm print, Gsteshead, 23-41

Taylor D.G., Inamdar R., and Bushell M-C., (1988). NMR imaging in theory and in practice. Phys. Med. Bio., Vol 33, No 6, 635-670

Thomas S.J., Wilkinson I.D., Dixon A.K. and Dendy P.P., (1992). Magnetic resonance imaging of Fricke-doped agarose gels for the visualization of radiotherapy dose distributions in a lung phantom. B.J.R, 65, 167-169 
Thwaites D.I., and Allahverdi M., (1997). The use of a semianatomic phantom in interdepartmental dosimetry audit. Proc World Cong. Med. Phys. Bio. Eng., Nice, P 998

Vasila M.S., Chakeres D.W., and Schmalbrock P., (1992). Optimizing imaging parameters and technical factors. In: Fundamentals of Magnetic Resonance Imaging. Williams \& Wilkins, USA

Vennart W., (1985). Principles of nuclear magnetic resonance. In: Physical principles and clinical applications of Nuclear Magnetic Resonance. Edited by Lerski R.A.. Paradigm print, Gsteshead, 1-21

Walker P., Lerski R.A., Mathure-De Vré R., Binet J., and Yane F., (1988). VI. Preparation of agarose gels as reference substances for NMR relaxation time measurement. Magn. Reson. Imaging. 6, 215-222

Webb S., (1993). The Physics of three-dimensional radiation therapy. Conformal radiotherapy, radio surgery and treatment planning. IOP Publishing Ltd

Webb S., (1998). Configuration options for intensity-modulated radiation therapy using multiple static fields shaped by a multileaf collimator. Phys. Med. Bio. 43, 241 260

White D.R., (1977). The formulation of tissue substitute materials using basic interaction data. Phys. Med. Biol. 22 889-899

White D.R., (1978). Tissue substitutes in experimental radiation physics. Med. Phys. $5(6), 467-479$ 
Wielopolski L., Maryanski M., Washington A.C., Schidlovsky G., Cohn S.H., Reinstein L.E., Kalend A.M., and Meek A.B., (1985). Polyacrylamide-based phantoms as tissue substitute in experimental radiation physics. Med. Phys 12(6), 788-792

Williams F., (1968). Principles of radiation-induced polymerization. In: Fundamental process in radiation chemistry. Edited by P. Ausloos. John Wiley \& Sons, Inc, USA.

Williams W. S. C., (1991). Nuclear and particle physics. Oxford Science publication $131-157$

Wong P.S., Clarke G.D., McColl R.W., Maryanski M.J., and Glatstein E., (1995). Imaging of radiation dose in two different types of gel-based dosimeters: A comparison between Fax and BANG. Med. Phys 22(6), 951

Young R.J., (1981). Introduction to polymers. Chapman \& Hall Ltd, USA.

Zhu T.C., Ding L., Liu C.R., Palta J.R., Simon W.E., and Shi J., (1997). Performance evaluation of a diode array for enhanced dynamic wedge dosimetry. Med. Phys 24(7), $1173-1180$ 